Florida International University FIU Digital Commons

\title{
Home-Based, Self-Administered Dyadic Cognitive Training for Healthy Older Adults: Feasibility Study
}

Natalia Shtompel

Florida International University, nshto001@fiu.edu

DOI: $10.25148 /$ etd.FIDC000718

Follow this and additional works at: https:// digitalcommons.fiu.edu/etd

Part of the Cognition and Perception Commons, and the Social Work Commons

\section{Recommended Citation}

Shtompel, Natalia, "Home-Based, Self-Administered Dyadic Cognitive Training for Healthy Older Adults: Feasibility Study" (2016). FIU Electronic Theses and Dissertations. 2606.

https://digitalcommons.fiu.edu/etd/2606 


\section{FLORIDA INTERNATIONAL UNIVERSITY}

Miami, Florida

\section{HOME-BASED, SELF-ADMINISTERED DYADIC COGNITIVE TRAINING FOR}

HEALTHY OLDER ADULTS: FEASIBILITY STUDY

A dissertation submitted in partial fulfillment of

the requirements for the degree of

DOCTOR OF PHILOSOPHY

in

SOCIAL WELFARE

by

Natalia Shtompel

2016 
To: Dean Tomas Guilarte

Robert Stempel College of Public Health and Social Work

This dissertation, written by Natalia Shtompel, and entitled Home-Based, Self-

Administered Dyadic Cognitive Training for Healthy Older Adults: Feasibility Study, having been approved in respect to style and intellectual content, is referred to you for judgment.

We have read the dissertation and recommend that it be approved.

$\begin{array}{r}\hline \text { Mark Macgowan } \\ \hline \text { Nicole Ruggiano } \\ \hline \text { Baul Gonzalez } \\ \hline\end{array}$

Date of Defense: June 28, 2016.

The dissertation of Natalia Shtompel is approved.

Dean Tomas Guilarte Robert Stempel College of Public Health and Social Work

Andrés G. Gil Vice President for Research and Economic Development And Dean of the University Graduate School

Florida International University, 2016 


\title{
ABSTRACT OF THE DISSERTATION \\ HOME-BASED, SELF-ADMINISTERED DYADIC COGNITIVE TRAINING FOR HEALTHY OLDER ADULTS: FEASIBILITY STUDY
}

by

\author{
Natalia Shtompel
}

Florida International University, 2016

Miami, Florida

\section{Professor Barbara Thomlison, Major Professor}

The negative effects of cognitive decline and impairment can be devastating for older adults and their families, and extremely costly for the healthcare system and the society. Cognitive training aims to maintain or improve cognition by utilizing repetitive tasks that target specific cognitive skills. The majority of cognitive training studies in healthy older adults involved home-based, individual, computerized approach or onsite, group, paperand-pencil format. These approaches may not be suitable for individuals with serious health or mobility issues, caregiving responsibilities, limited transportation, or limited computer or internet access. A novel, home-based, self-administered cognitive training intervention was developed to address these barriers. It involves two older adults taking turns to administer paper-and-pencil tasks to one another. The purpose of the study was to evaluate feasibility and participant acceptability of this novel approach to cognitive training. Eighteen participants (9 dyads) 65-91 years $(M=75.94, S D=7.66)$ underwent assessment and began intervention. Assessment included measures of cognitive skills and self-reported quality of life, health status, and daily functioning. Four dyads were married couples who had sessions at home. Other dyads met at various public locations and 
included friends, neighbors, or unfamiliar older adults connected by the researcher. Fourteen participants (7 dyads) completed cognitive training intervention that included 924 sessions $(M=15.14, S D=5.30)$ over $4-21$ weeks $(M=12.21, S D=5.44)$, postintervention assessment, and detailed interviews. Quantitative data demonstrated that the sample did not decline on any cognitive measures and exhibited improvement on visuospatial skills and delayed visual memory (Cohen's $d=.67 \&-1.10$ ). Additional analyses revealed that the results were mainly attributable to improvement in females (Cohen's $d=-1.84 \&-1.35$ ), who demonstrated weaknesses in these cognitive skills at baseline. The participants reported that the dyadic approach was flexible, convenient, and enjoyable. They also provided valuable feedback and suggestions for modifying the content and other aspects of the intervention. The findings suggest that dyadic cognitive training is feasible and well-received by older adults. Those with weaknesses in cognitive domains may show larger gains in respective domains and benefit most from cognitive training. 
C Copyright 2016 by Natalia Shtompel

All rights reserved. 


\section{TABLE OF CONTENTS}

CHAPTER

PAGE

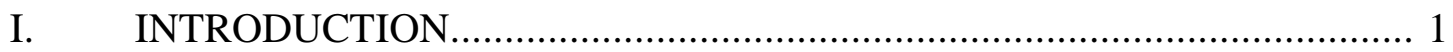

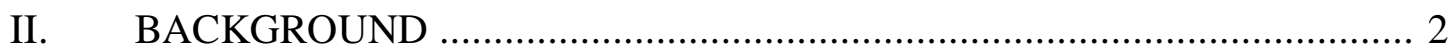

Age-Related Cognitive Decline .............................................................. 2

Negative effects ................................................................... 5

Individuals and families............................................... 5

Healthcare system and the society …………………...... 8

Risk and protective factors ..................................................... 9

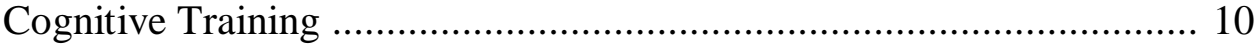

Theoretical frameworks ...................................................... 12

Psychosocial frameworks ........................................... 12

Cognitive frameworks ................................................ 13

Neurophysiological frameworks .................................... 14

Previous studies on cognitive training ..................................... 19

Randomized controlled trials ........................................ 19

Summary of findings .................................................. 34

Criticisms of cognitive training....................................... 36

Peer-based interventions .......................................................... 37

Collaboration in cognitive training ................................ 38

Social Factors and Cognition ............................................................ 42

Summary and Statement of the Problem .............................................. 43

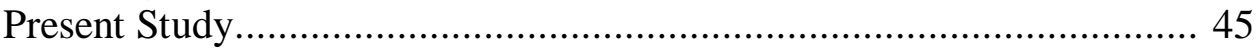

Purpose

Conceptual framework ......................................................... 45

Practical and economic reasons ................................................ 47

Research questions and hypotheses ............................................ 49

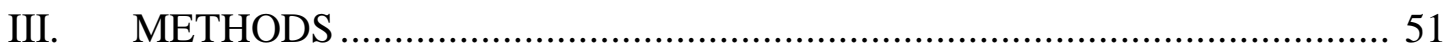

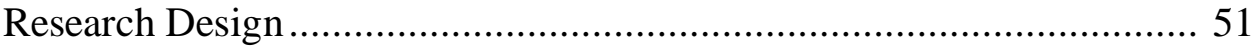

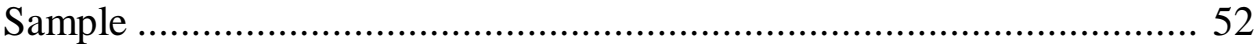

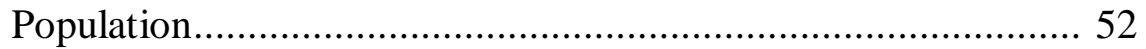

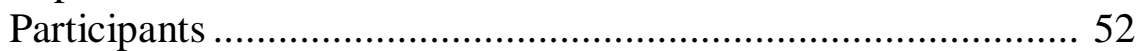

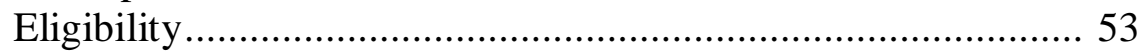

Inclusion criteria............................................... 53

Exclusion criteria....................................................... 54

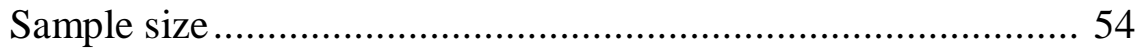

Tests and Measurements ................................................................. 55

Screening measures................................................................. 55

Slosson Oral Reading Test- Revised …………………. 55

Mini-Mental State Examination ..................................... 56

Patient Health Questionnaire (Depression)....................... 57

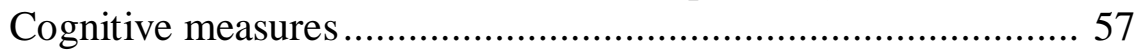


Stroop Color and Word Test ......................................... 58

Trail Making Test ………………………………….... 58

Repeatable Battery for the Assessment of the

Neuropsychological Status- Update ................................ 59

Self-report measures …………………….................................. 61

World Health Organization Quality of Life- Older

Adult ……................................................................ 61

Research and Development 36-Item Short-Form Health

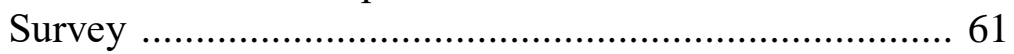

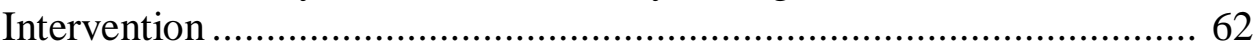

Bayer Activities of Daily Living ................................... 61

Setting, frequency, and duration............................................... 62

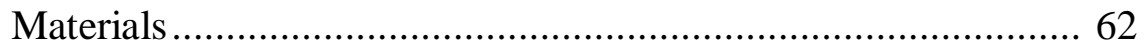

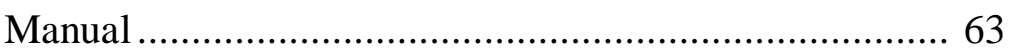

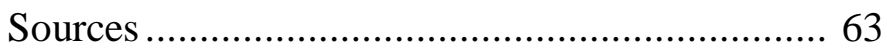

Task adaptation.................................................. 66

Functions targeted.............................................. 67

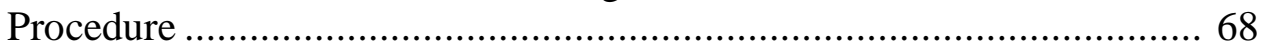

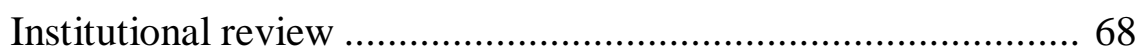

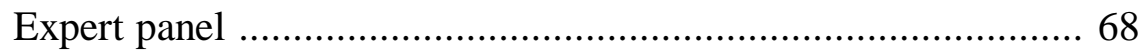

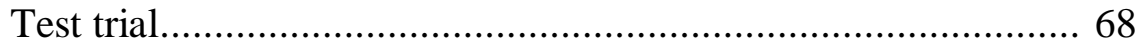

Participant recruitment ............................................................. 69

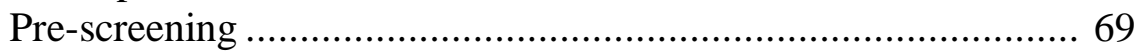

Screening and pre-intervention assessment.................................. 71

Intervention.................................................................... 72

Post-intervention assessment.................................................. 73

Post-intervention interviews........................................................ 73

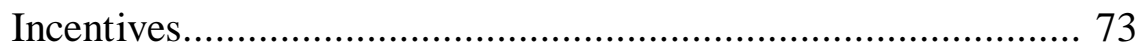

Data collection and processing .................................................. 73

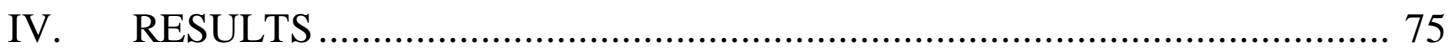

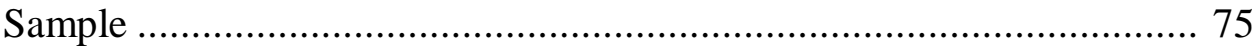

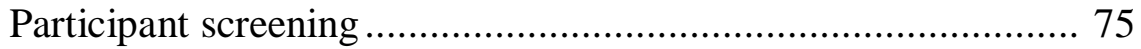

Demographic characteristics ………………………............. 75

Participant retention ................................................................. 76

Participant adherence ............................................................ 78

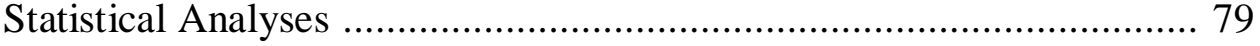

Data Screening ………................................................................ 79

Retention and Adherence Variables ................................................. 80

Pre-Intervention Assessment........................................................... 83

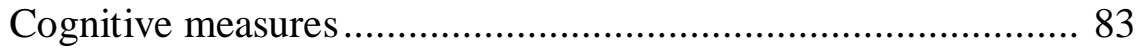

Demographic variables .............................................. 91

Self-report measures ................................................................ 93

Demographic variables ................................................. 95

Post-Intervention Assessment ………………………....................... 96

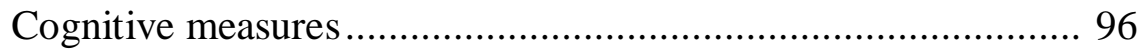




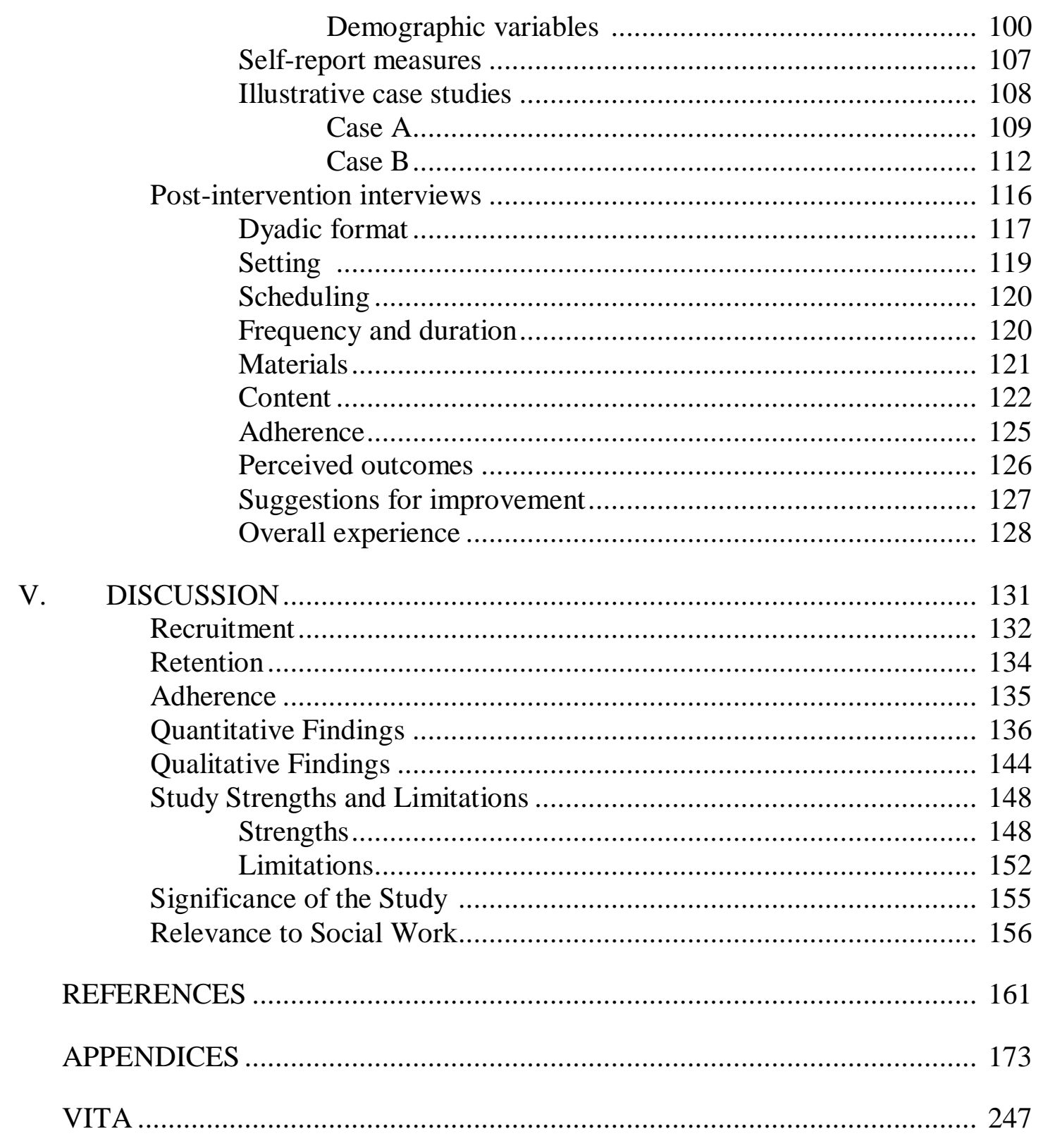




\section{LIST OF TABLES}

TABLE

PAGE

1. Description of Cognitive Functions and Corresponding Measures

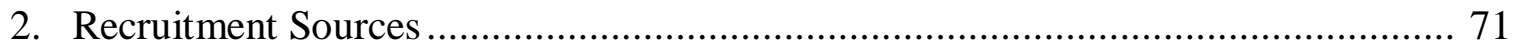

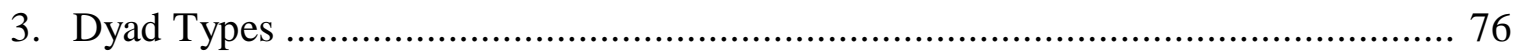

4. Descriptive Statistics for Cognitive Test Scores at Pre-Intervention Assessment .... 85

5. Bivariate Correlations among Cognitive Measures .......................................... 95

6. Descriptive Statistics for Self-Report Measures.......................................... 93

7. Descriptive Statistics for Cognitive Test Scores at Pre-Test and Post-Test and

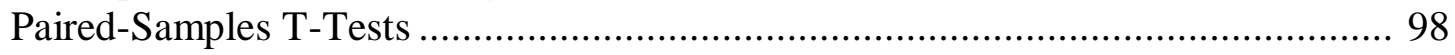

8. Numbers of Participants Who Demonstrated Increase, Decrease, or No Change on Cognitive Test Scores ........................................................................ 105

9. Each Participant's Changes in Scores on Cognitive Measures. ............................ 107

10. Qualitative Descriptors for Scaled and Domain Scores................................... 108

11. Qualitative Descriptors for Repeatable Battery for the Assessment of Neuropsychological Status (RBANS-U) Subtest Percentile Ranges

12. Case A: Pre-Test and Post-Test Cognitive Scores.......................................... 111

13. Case B: Pre-Test and Post-Test Cognitive Scores ......................................... 115 


\section{LIST OF FIGURES}

FIGURE

PAGE

1. Conceptual Framework ........................................................................... 47

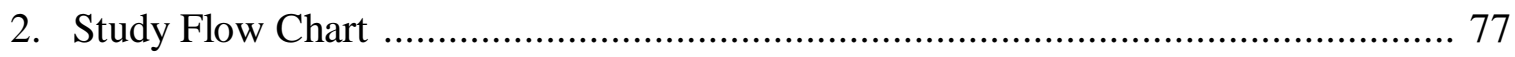

3. Frequency Distribution for Total Score of the Repeatable Battery for the Assessment of Neuropsychological Status-Updated (RBANS-U)

4. Sample Means for the Five Domains of the Repeatable Battery for the Assessment of Neuropsychological Status-Update (RBANS-U).

5. Pre-Test and Post-Test Means for Repeatable Battery for the Assessment of Neuropsychological Status-Update (RBANS-U)

6. Males and Females' Scores on the Visuospatial Domain of the Repeatable Battery for the Assessment of Neuropsychological Status-Update (RBANS-U).

7. Males and Females' Scores on the Figure Recall Subtest of the Repeatable Battery for the Assessment of Neuropsychological Status-Update (RBANS-U). 


\section{INTRODUCTION}

With increases in life expectancy, the proportion of older adults in the US continues to grow (Administration on Aging, 2016). Cognitive decline has become an important area of concern in gerontology, along with physical illnesses and mental health issues (National Institute on Aging, 2009). The general public is concerned about their cognitive health (Connel, Roberts, \& McLaughlin, 2007), and older adults view cognitive vitality as an important part of successful aging (Laditka et al., 2009). Cognitive stimulation has been found to reduce the risk of cognitive decline, in addition to physical activity, social engagement, and healthy lifestyle (Baumgart et al., 2015). In line with these findings, a number of cognitive training approaches have been evaluated, although this area of research is still relatively sparse.

Technology industry has responded to the potential benefits of increased mental stimulation by developing commercial computerized programs to train memory and other cognitive skills (Lampit, Valenzuela, \& Gates, 2015). Exaggerated claims lacking empirical support with regard to efficacy of these computer programs (Stanford Center on Longevity, 2014) have led to a deceptive advertising lawsuit against one of the most popular brain games platform, which was settled (Federal Trade Commission, 2016). Although the results of computerized approaches to cognitive training have been promising (see meta-analysis by Lampit, Hallock, \& Valenzuela, 2014), such format of intervention delivery may not be suitable for older adults who have limited access to computer and/or internet or are uncomfortable using it, or simply prefer using paper and pencil format. A number of group paper-and-pencil cognitive training programs facilitated by trained professionals onsite have also demonstrated promising results with 
long-lasting effects (e.g., Rebok et al., 2014), but it is not clear how these laboratorybased programs can be translated into community services. There is a need to develop and evaluate paper-and-pencil cognitive engagement programs that are low-cost, flexible, and that can be easily implemented in community settings. Such programs may be included in existing community services along with physical fitness and recreational activities, with the goal of incorporating cognitive health into the overall model of promoting general health, well-being, and successful aging. The present study is a preliminary trial evaluating feasibility and participant acceptability of a novel, flexible, self-administered, dyadic cognitive stimulation program for community-dwelling older adults without Mild Cognitive Impairment (MCI) or dementia.

\section{BACKGROUND}

\section{Age-Related Cognitive Decline}

In the American Psychological Association (APA) guidelines for evaluating dementia and cognitive decline, age-related cognitive decline (also referred to as natural or normal cognitive decline) is described as an expected degree of decay in cognitive efficiency (APA, 2011). It is considered to be a natural consequence of aging processes, as it is "usually not pathological, but rather parallels a number of common diseases in physiological function that occur in conjunction with normal developmental process" (para. 4).

Age-related cognitive decline is differentiated from pathological forms of cognitive changes caused by Alzheimer's disease and other dementias, as well as from MCI, a pre-clinical stage of dementia (APA, 2011). However, normal cognitive decline has been found to be associated with an increased risk of developing cognitive 
impairment and dementia, which suggests that normal cognitive decline and cognitive impairment are distinct yet related phenomena (Plassman, Williams, Burke, Holsinger, \& Benjamin, 2010; Schaie, Willis, \& Caskie, 2004). According to brain imaging studies, natural cognitive decline is associated with white matter hyperintensities (Wakefield et al., 2010), decreased cerebral perfusion (blood flow) in certain areas of the brain (Chao \& Weiner, 2010), and reduced lateral frontal grey matter volume and frontal cortical thickness (Gautam, Cherbuin, Sachdev, Wen, \& Anstey, 2011). In addition, animal models and human studies have shown that increased inflammation and activation in microglia can lead to impaired regulatory systems, causing cognitive and depressive symptoms. Despite these findings, the mechanisms underlying age-related cognitive decline remain unclear (Norden \& Godbout, 2013).

Specific statistics with regard to the incidence and prevalence of age-related cognitive decline are unavailable, but the literature indicates that the vast majority of older adults demonstrate some decline in cognitive functions, and the risk of cognitive decline increases with age. Fortunately, cognitive decline in healthy older adults is not inevitable, as some older individuals continue to exhibit cognitive vitality well into old age (Park, O'Connell, \& Thomson, 2003). A number of older adults over the age of 80 (sometimes referred to as Super-Agers), not only perform comparably to those aged 5065 years, but also tend to have higher cortical volume and less cortical atrophy compared to their peers with normal cognitive decline (Harrison, Weintraub, Mesulam, \& Rogalski, 2012). Additionally, studies of centenarians have shown that the majority of older adults in their late 90s and 100s still reside in the community, have few cognitive limitations, and report low depression/psychological distress rates and high life satisfaction (e.g., 
Jopp, Park, Lehrfeld, \& Paggi, 2016; Sachdev et al., 2013). Such findings point to the possibility of maintaining or improving cognition with age through preventative and intervention efforts.

In terms of its onset and progression, longitudinal data largely demonstrate that age-related cognitive decline occurs after the age of 60 years (see review by Hedden \& Gabrieli, 2004), with some evidence pointing to cognitive decline in individuals in their 40s and 50s (Singh-Manoux et al., 2012). Cross-sectional studies reveal more linear, lifelong declines in cognitive functions (Hedden \& Gabrieli, 2004), which, however, are likely influenced by cohort and educational differences (Hedden \& Gabrieli, 2004; SinghManoux et al., 2012; Zelinski, Dalton, \& Hindin, 2011). It has also been found that cognitive decline accelerates with age (e.g., Singh-Manoux et al., 2012). It should be noted that heterogeneity of definitions, measures, samples, and other methodological factors among studies, individual differences in cognitive decline, as well as the complexity of the underlying age- and disease-related neurobiochemical changes make it difficult to quantify the rate of cognitive decline and differentiate between age-related cognitive decline and early stages of pathological cognitive decline (Park et al., 2003; Raz \& Lindenberger, 2011; Schaie, Willis, \& Caskie, 2004).

There is, however, evidence showing that some cognitive functions are more prone to age-related decline than others. According to Kramer \& Willis (2002), fluid abilities, which refer to process-based skills involving immediate tasks at hand, are more likely to decline with age compared to crystallized abilities, or knowledge-based skills that rely on previously learned information. The distinction between fluid and crystallized abilities is important because individuals typically rely on their fluid skills in their daily 
activities, and these are the skills that tend to decline with age. Fluid abilities include working memory, attention, reasoning, and visuospatial skills (Hedden \& Gabrieli, 2004; Kramer \& Willis, 2002). The speed of information processing and reaction time also decrease with age, as well as executive control or executive functioning processes which include response inhibition, planning, organization, and shifting mental sets (Zelinski et al., 2011). On the other hand, such knowledge-based skills as language and vocabulary, and semantic and autobiographic memory remain relatively intact in older age (Hedden \& Gabrieli, 2004; Kramer \& Willis, 2002). With regard to subjective complaints, older individuals with age-related cognitive decline typically report memory problems including difficulty remembering names, conversations, things to do, and finding words ("tip of the tongue"). Some research suggests that memory problems are generally due to information encoding (rather than retrieval) failures and can largely be attributed to, and are preceded by decline in executive functions (Carlson, Xue, Zhou, \& Fried, 2009). Of note, deficits in sensory perception and depressive symptoms may affect cognition in older individuals and thus, need to be considered when assessing cognitive status in this population (Blazer, 2009; Zelinski et al, 2011).

Negative effects. Cognitive decline has been linked to a range of negative effects in older adults. However, the implications of age-related cognitive decline are not limited to older adults who are experiencing cognitive decay. Caregivers, healthcare system, economy, and consequently, the society at large are affected by age-related cognitive decline.

Individuals and families. According to Zelinksi and co-authors (2011), declines in executive functions, attention, working memory, and processing speed may affect older 
adults' judgment, ability to solve problems, orientation, remembering information and future tasks, and reacting to stimuli. In their turn, these deficits may translate into difficulties in older adults' lives. For instance, it has been found that cognitive decline may affect healthy older adults' driving skills, which may impact their safety, health, and independence, as well as ability to attend doctors' appointments and engage in leisure and other activities (Kowalski et al., 2011; McKnight \& McKnight, 1999).

Falls with subsequent injuries and disability is another serious safety issue in the aging population. Herman, Mirelman, Giladi, Schweiger, \& Hausdorff (2010) found that poorer executive functioning skills were related to higher risk of future falling in healthy older adults. Muir, Gopaul, \& Montero Odasso (2012) performed a meta-analysis of multiple studies on fall risk and found that global cognitive status was related to serious injury due to a fall, and deficits in executive functions were strongly associated with an increased fall risk in non-demented adults 60 years old and older. Moreover, in their prospective study, Boyle and colleagues (2012) found that cognitive decline led to poor decision making and increased susceptibility to scams in healthy older adults.

In addition to an increased risk of driving accidents and falls and poor decisionmaking, age-related cognitive decline has been linked to problems in performing activities of daily living (ADLs). For instance, Montejo, Montenegro, Fernandez, \& Maestu (2012) found that participants who reported memory problems were more likely to have trouble using telephone, managing medications, and participating in social activities $(N=1637)$. Tabbarah, Crimmins, \& Seeman (2002) found that in their sample of 1189 healthy adults aged 70-79 years, cognitive decline was associated with a decreased ability to perform routine and novel physical tasks, such as signing one's name 
or standing on one leg. Infurna, Gerstorf, Ryan, \& Smith (2011) evaluated a sample of 6,990 70-95 year olds and found that memory problems predicted functional limitations in older adults in such tasks as shopping, cooking, walking, and lifting/picking up objects. Overall, executive functions seem to have the strongest association with functional limitations and disability in older adults compared to other cognitive skills, particularly when it comes to more complex, instrumental activities of daily living (IADLs; Royall et al., 2007). The link between performance of IADLs and cognition is bidirectional, as illustrated by the finding that decline in IADLs may be an early marker of dementia (Castilla-Rilo et al., 2007), up to 10 years before clinical diagnosis (Pérès et al., 2008).

Performance of ADLs is not only linked to cognition, but also to depression in older adults. Moreover, the relationship between cognitive functioning and depression also appears to be bidirectional. Longitudinal research shows that problems in ADLs may lead to late-onset depression or exacerbate existing depressive symptoms (Yang \& George, 2005). In its turn, depression has been found to be a risk factor for cognitive decline and dementia, in addition to a wide range of health problems, disability, mortality, and poor illness recovery (Barry, Murphy, \& Gill, 2011; Blazer, 2009; Emery, 2011). Thus, age-related cognitive decline may indirectly affect emotional and health status in older individuals.

Given the impact of cognitive decline on older persons' daily lives, the finding that it also affects their well-being is not surprising. For example, subjective memory complaints were linked with lower quality of life in healthy older adults in some studies (e.g., Montejo et al., 2012; Pusswald et al., 2015), and cognitive functioning was related 
to overall well-being (Jones, Rapport, Hanks, Lichtenberg, \& Telmet, 2003). Moreover, Calero and Navarro (2011) found that cognitive functioning may be a stronger predictor of well-being and independence in older adults compared to age.

Since cognitive decline may reduce older adults' ability to perform routine daily activities and limit their independence, it may also affect their caregivers' quality of life. Spouses, children, and other family members of older adults with cognitive decline may experience increased stress and emotional and financial difficulties (Infurna et al., 2011). They may be required to become more involved with older persons' care and take time from work or other tasks in order to assist their loved ones. As a result, family members' quality of life may be compromised, since amount of time caregiving is related to caregiver burden, which in turn affects caregiver well-being (Chappell \& Reid, 2002).

Healthcare system and the society. Collectively, individual and caregiver biopsycho-social factors translate into increased costs for healthcare and community services and the economy at large, ultimately resulting in higher burden on the society. Older adults with cognitive decline may have to retire earlier and may need more community resources such as nutrition and transportation services. In addition, they may require high-cost healthcare services due to falls, injuries, accidents, or medication mismanagement (Infurna et al., 2011; Park et al., 2003). As an example, the National Center for Injury Prevention and Control, Centers for Disease Control and Prevention (2014) estimates that total medical expenditures for older adults' fall injuries were 30 billion in 2010. As for driving accidents and susceptibility to scams, data show an increase in liability and collision claims for adults over the age of 65 years (Insurance Institute for Highway Safety, 2014), and an estimated 2.9 billion in annual cost of 
financial abuse of seniors (MetLife Mature Market Institute, 2011). The finding that difficulties in ADLs may contribute to depression (Yang \& George, 2005) is also relevant here since older adults with depressive symptoms utilize about 50\% more healthcare services of all types compared to non-depressed older patients (Katon, Lin, Russo, \& Unützer, 2003). Thus, maintaining cognitive functioning or delaying the onset of cognitive decline among older individuals may translate into reduced economic and healthcare costs.

Risk and protective factors. As previously discussed, age-related cognitive decline is not inevitable, as there is evidence showing that some older adults demonstrate minimal cognitive decline well into later stages of their lives (National Institute on Aging, 2009; Park et al., 2003). These findings, combined with the negative effects of cognitive decline have fueled research examining risk and protective factors for cognitive decline in older adults that could point to potential interventions. For instance, there is evidence that involvement in such activities as volunteering, recreation/sports, gardening and other domestic chores predicts stronger cognitive abilities with age (Newson \& Kemps, 2005). Similarly, higher social integration and social engagement was found to contribute to better cognitive outcomes in older people (Zunzunegui, Alvarado, Del Ser, \& Otero, 2003). Observational longitudinal studies have shown that healthy lifestyle (healthy diet and exercise), as well as engaging in cognitively stimulating tasks also contribute to cognitive fitness in this population (see reviews by Baumgart et al., 2015; Plassman et al., 2010). At the same time, early retirement has a negative impact on cognitive functioning among older adults, likely due to reduced mental stimulation (Rohwedder \& Willis, 2010). Among the risk factors for cognitive decline in older adults without MCI or 
dementia are also tobacco use, diabetes, metabolic syndrome, obesity, and depression. Although there is a genetic risk factor (apolipoprotein E e4 genotype), it plays a relatively small role compared to modifiable, lifestyle risk factors for cognitive decline (Baumgart et al., 2015; Plassman et al., 2010). Interestingly, no association was found between cognition and supplements such as vitamins B, C, and E, beta-carotene, folate, and Ginkgo biloba, or medications for high blood pressure, Statins, gonadal steroids, nonsteroidal anti-inflammatory drugs, or cholinesterase inhibitors (Plassman et al., 2010).

A number of studies have evaluated various interventions for age-related cognitive decline. Non-pharmacological interventions typically involve physical exercise or cognitive training programs, or a combination of both. There is some evidence that physical exercise interventions are effective in improving cognition in older adults, although the gains tend to be limited to specific domains, particularly executive functions, attention, and processing speed. The type and duration of intervention, duration of sessions, and some demographic factors may mediate efficacy of physical fitness interventions (see meta-analyses by Angevaren, Aufdemkampe, Verhaar, Aleman \& Vanhees, 2008; Colcombe \& Kramer, 2003). The following section focuses on the other type of non-pharmacological intervention for cognitive decline, cognitive training.

\section{Cognitive Training}

Cognitive training aims to maintain or improve cognitive functioning and involves repeated practice of standardized tasks that target specific cognitive domains (Gates, Sachdev, Singh, \& Valenzuela, 2011). In healthy older adults, the ultimate goal of cognitive training is to maintain performance of ADLs and IADLs by addressing agerelated cognitive decline (Ball, Ross, Roth, \& Edwards, 2013). Cognitive training may 
utilize restorative approach, compensatory approach, or a combination of both.

Restorative training aims to improve cognitive abilities and typically includes systematic exercises that target narrow cognitive skills (Kurz, Leucht, \& Lautenschlager, 2011; NIH, 1998). For example, individuals with a processing speed deficit may practice simple visual search task that requires finding a specific symbol among other symbols as fast as possible. On the other hand, compensatory cognitive training focuses on helping individuals adapt to their cognitive deficits and utilize their strengths, as they learn various techniques and strategies to approach cognitive tasks and practice the use of external aides to improve cognitive efficiency (Kurz, et al., 2011; NIH, 1998). Examples of the compensatory approach include using associative memory to pair names with faces in order to remember them, or writing things to do on post-it notes (Rebok, Parisi, Gross, $\&$ Spira, 2010). In both restorative and compensatory training, exercises and activities may be presented verbally or visually, and be paper and pencil-based or computer-based. Training may target a single or multiple cognitive functions, occur in group or individual settings, and be self-administered or facilitated by a trained professional (NIH, 1998; Williams \& Kemper, 2010).

Initially developed for patients with traumatic brain injury, where it is typically referred to as cognitive rehabilitation (National Institutes of Health [NIH], 1998), cognitive training interventions have become increasingly used in other populations, including patients with multiple sclerosis, stroke, Parkinson's disease, and schizophrenia. Additionally, cognitive training interventions have been employed in many studies targeting individuals with MCI and dementia. According to Kurz, Leucht, and Lautenschlager (2011), this line of research is important because medications targeting 
cognitive decline in patients with MCI or dementia may have detrimental side-effects, as well as side-effects caused by interaction with other drugs. The authors performed a systematic review of experimental studies on cognitive training in MCI and dementia and found that the results have been inconsistent. Overall, cognitive training yielded only modest gains in cognitive functions, which, however, were comparable to the efficacy of pharmacological interventions. Importantly, several studies revealed that cognitive training delayed the onset of cognitive deterioration, improved performance of daily activities, and enhanced the MCI and dementia patients' ability to attain their personal goals (Kurz et al., 2011). Another systematic review of cognitive interventions for cognitive decline by Gates et al. (2011) focused on MCI patients only and found that there were moderate-to-large effects on memory outcomes in this population. The authors found that administering cognitive exercises to individuals with MCI led to better results on memory domains than teaching them memory strategies, suggesting that restorative cognitive training may be more beneficial in this population compared to compensatory cognitive training (Gates et al., 2011). Since the population of interest in the present study is cognitively healthy older adults, the following sections focus on theories of nonpathological cognitive aging and the mechanisms behind cognitive training, as well as existing studies on cognitive training in older adults without MCI or dementia.

\section{Theoretical frameworks.}

Psychosocial frameworks. Among the psychosocial theories relevant to cognitive aging is the classic social theory of Disengagement, which postulates that as people age, they face gradual separation from the society due to inevitable and irreversible decrease in involvement in work force, family, and social life (Perzynski, 2006). It can be inferred 
that older individuals' cognitive decline is a part of overall decline in their professional and social roles. The disengagement theory has been criticized for its overly generalized and pessimistic view of seniors. It has been refuted by numerous studies on successful aging and is generally considered outdated (Perzynski, 2006).

A more current sociological framework, Life Course Perspective, takes into account the effects of cultural and societal factors (institutions, shared life experiences, behaviors and attitudes etc.) on the lives of groups or cohorts of individuals. In the light of epidemiological studies on aging, life course theory provides a framework for understanding the cumulative effects of educational opportunities, income, social engagement, and physical environment on individuals' health outcomes, including cognitive functioning in later life (Health and the Life Course, 2001). Specifically, certain lifestyle and health variables linked to cognitive decline such as diet, exercise, cognitive stimulation, tobacco use, diabetes, metabolic issues, and depression (Baumgart et al., 2015; Plassman et al., 2010) may be related to individuals' socio-economic situation, which points to the importance of broad, systems-based interventions (Glymour, 2011).

Cognitive frameworks. The Disuse theory of cognitive aging, also referred to as "use it or lose it" perspective, parallels the disengagement theory of aging. According to the disuse theory, older adults perform increasingly worse on fluid-based tasks because older adults decrease the use of their fluid cognitive skills with age and thus, lack recent experience in this domain (Salthouse, 1999). Because the disuse theory is difficult to test experimentally, there is little evidence supporting it (Park \& Bischof, 2013). However, this theory appears plausible and is quite popular among clinicians and the general public (Salthouse, 1999). Moreover, the results of a longitudinal study on retirement and 
cognitive decline across 13 Westernized countries (including the US) by Rohwedder and Willis (2010) suggest that there is a causal relationship between early retirement and cognitive decline in older adults. The authors believe that major lifestyle changes associated with retirement, including reduced cognitive stimulation lead to an increased risk of age-related cognitive decline (Rohwedder \& Willis, 2010).

Another view on cognitive decline in older adults is the Specific-Deficit Hypothesis, which holds that age-related cognitive decline occurs due to diminished efficiency and effectiveness of core processing operations or components that play a role in virtually all cognitive tasks (Salthouse, 1999). Specifically, decline in such cognitive skills as automatic or controlled attention, encoding, and working memory contributes to weaknesses in other fluid (process-based) skills, which are consequently prone to decay with age (Salthouse, 1999). This theory seems plausible in the context of research showing that fluid cognitive skills tend to decline in older adults, as opposed to crystallized (knowledge-based) abilities that are relatively stable (Kramer \& Willis, 2002). Even though there is some research supporting the specific-deficit hypothesis, it fails to describe how and why the observed weakening in the processing operations takes place with age (Salthouse, 1999).

Neurophysiological frameworks. A neurophysiological theory that helps explain the mechanisms behind age-related decline in fluid abilities is the Prefrontal Cortex Function Theory (West, 1996). According to this view, cognitive decline occurs due to structural and functional changes in the prefrontal cortex, a region of the brain linked to fluid abilities and executive functions. Among the changes in the prefrontal cortex is volume reduction, which occurs earlier and is more pronounced in prefrontal cortex than 
other regions of the brain. Specifically, a volume reduction of 10-17 percent is observed in the prefrontal cortex between ages $50-70$ years, while the volume is reduced by only about one percent in other cortical regions of the aging brain. This volume reduction in prefrontal cortex is attributed to decreased size of individual neurons due to the loss of dendrite extensions, rather than actual neuronal loss. Atrophy in neuronal dendrites leads to reduced synaptic activity among neurons, resulting in decreased brain connectivity and efficiency (West, 1996).

West (1996) explains that in addition to volume reduction, there are declines in the concentration of some neurotransmitters (i.e., dopamine, norepinephrine, and serotonin) and their receptor sites, as well as decreased oxygen utilization, all of which are more pronounced in the prefrontal cortex. These findings suggest that prefrontal cortex is more vulnerable to age-related brain changes and the declines in fluid abilities result from structural and neurochemical changes in this region of the brain (West, 1996). A related concept describing the progression of brain changes is referred to as "last in, first out," the idea that the circuitry that is last to undergo myelination during childhood and adolescence (prefrontal cortex) is the first one to exhibit deterioration (Reuter-Lorenz \& Park, 2010).

According to Reuter-Lorenz \& Park (2010), neuroimaging studies have provided evidence for a range of differences between younger and older adults' brain functioning. For instance, older adults display overactivation in brain regions directly and indirectly involved during specific tasks. One explanation for this phenomenon is that older adults compensate for weaker skills by increased activation of the related region and/or by involving other regions, regardless of whether this compensation improves actual 
performance. Prefrontal compensation is commonly observed in older individuals, which seems paradoxical as frontal regions are more susceptible to age-related atrophy. A related concept of "dedifferentiation" has emerged from the finding that older adults demonstrate loss in regional specificity when performing certain tasks. CompensationRelated Utilization of Neural Circuits Hypothesis (CRUNCH) is a theory explaining overactivation and compensation in older adults. According to this hypothesis, older adults utilize extra circuits on easier tasks where younger adults' activation is more focal; however, with more difficult tasks, older adults show underactivation as they already used up their neural resources, whereas younger adults increase their brain activation (Reuter-Lorenz \& Park, 2010).

Scaffolding Theory of Aging and Cognition (STAC) provides a broader view of the age-related brain changes. According to this theory, the brain responds to various alterations in its structure and function by "scaffolding" alternative neural circuits, which allows healthy older individuals maintain high level of cognition that is sufficient to support their daily activities (Reuter-Lorenz \& Park, 2010). The efficacy of scaffolding mediates the brain's response to neural atrophy and may be affected by physical health, cognitive stimulation, and new learning. An important aspect of STAC is that scaffolding is acquired throughout lifespan in response to stress and other challenges to which the brain has to adapt. As a result, an individual's scaffolding trends in older age may be a reflection of lifelong adaptation mechanisms (Reuter-Lorenz \& Park, 2010).

Neuroplasticity is a related concept which provides a general framework for explaining structural and functional brain changes due to experience, including cognitive training. According to Slagter, Davidson, and Lutz (2011), it has become widely accepted 
in the medical and neuroscientific community that human brain is plastic and is able to reorganize, rebuild, or create new neuronal circuits. Brain plasticity is an "intrinsic property of the nervous system retained throughout lifespan and the obligatory consequence of all neural activity, including mental practice" (p. 1). Adult brain, including the aging brain, can undergo changes in its function and structure as a result of experience and/or environment, and not only sensory input and physical acts, but also each thought process may contribute to changes in brain circuitry underlying complex mental processes. Importantly, these changes may occur as a result of both long-term exposure to stimuli and a relatively short-term practice (Slagter et al., 2011).

Unfortunately, the vast majority of cognitive training RCTs (reviewed in the Previous Studies section) lack neuroimaging data to supplement their cognitive test findings. However, several studies provide initial evidence for neurophysiological changes following cognitive training interventions that align with the concept of neuroplasticity and the STAC theory (Park \& Bischof, 2013). For instance, some findings point to changes in brain activation after cognitive training. Brehmer et al. (2011) employed 5-week computerized training of working memory in a sample of 23 older adults (60-70 years old). Intervention group received high intensity working memory training that adjusted its difficulty to each individual's performance, while the active control group received the same working memory training but at low intensity. Both groups demonstrated decreased levels of activation during working memory tasks in frontal, parietal, and occipital cortical brain regions after the training; however, this was more pronounced in the intervention group. Additionally, increases in activation of certain subcortical regions were observed post-intervention, where larger increases were 
associated with larger gains on cognition tests. The unique design of the study, where the same intervention with high and low levels of intensity was introduced to two groups, provides evidence of dose-response relationship, since higher "dosage" of intervention led to larger changes in brain activation. The authors (Brehmer et al., 2011) suggest that older adults' decreased brain activation was due to lower levels of effort and attention required for completion of tasks, pointing to increased cognitive efficiency.

Another study (Carlson et al., 2009) highlighted the benefits of increased engagement among a sample of 17 older adults with low education and low overall cognitive status. Eight of the older adults participated in a volunteer social service program at elementary schools, and nine were in the wait-list control group. The results revealed that the intervention group had increases in activation of prefrontal cortex and anterior cingulate cortex, which was accompanied by gains in executive control (inhibition). These imaging results show that not only targeted cognitive training, but also increased general mental stimulation may lead to neurological brain changes through neuroplasticity. The findings also provide support for the existing body of literature that links social engagement with lower risk of age-related cognitive decline (e.g., Rohwedder \& Willis, 2010).

There is also evidence of slowed volume reduction following cognitive training. Lövdén et al. (2012) employed training of visuo-spatial ability in a sample of 47 older men aged 60-70 years and 44 younger men aged 20-30 years. During the 4-month training, the intervention group was exposed to navigation training on screen while walking on a treadmill, while the frequency and duration-matched control group engaged in walking on the treadmill without the navigation tasks. After the training, both younger 
and older adults in the control group exhibited expected levels of hippocampal volume reduction, while younger and older adults in the intervention group showed no decline in their hippocampal volume. The same pattern was observed at the 4-month follow-up (Lövdén et al., 2012). Although few studies on cognitive engagament incorporated neuroimaging findings, emerging data support the hypothesis that improvements in cognition may stem from underlying changes in the cortex. Long-term cognitive training interventions are reviewed in the following section.

Previous studies on cognitive training. A number of quasi-experimental studies and randomized controlled trials (RCTs) on cognitive training in healthy older adults have been conducted to date. Existing quasi-experiments typically employed various strategies to improve memory in healthy older adults including collaboration with others (Henkel \& Rajaram, 2011), method of loci and strategic training (Cavallini, Pagnin, \& Vecchi, 2003), self-referencing and other-referencing, semantic encoding and structural encoding (Hamami, Serbun, \& Gutchess, 2011). The results demonstrated that these strategies were somewhat beneficial in improving recall in healthy older adults, who were able to benefit from the use of memory techniques comparably with younger adults. These studies indicate that compensatory strategies can enhance verbal memory performance in older adults, which is central to their daily functioning and is among the functions most vulnerable to cognitive decline (Park et al., 2003; Schaie et al., 2004).

Randomized controlled trials. Several RCTs targeting age-related cognitive decline have yielded promising results. The largest paper-and-pencil RCT with the longest follow-up period to date, was a 2006 multi-site ACTIVE (Advanced Cognitive Training for Independent and Vital Elderly; Willis et al., 2006). A diverse sample of 2,802 
healthy adults 65 years of age and older living independently in Pennsylvania were recruited from community and clinical settings. Eligible participants were randomly assigned to one of the three intervention groups including reasoning, memory, and processing speed training groups, or an assessment-only control group. Reasoning group focused on identifying patterns, memory group was trained on how to use memory strategies, and processing group was trained to search for items on a computer screen. The participants in the three treatment groups underwent 10 intervention sessions in small groups on site over the course of five or six weeks. Booster sessions were conducted 11 and 35 months post-treatment among a random subsample of participants. Individuals were assessed pre- and post-treatment, and at 1,2,3, and 5, and 10-year follow-up assessment sessions (Rebok et al., 2014; Willis et al., 2006).

Eighty nine percent of older adults completed the intervention. The results showed that at post-treatment assessment, the participants improved their performance on the trained cognitive functions (Willis et al., 2006). In other words, each of the three treatment groups showed significant improvement in their trained domain, but not other domains, which confirmed that the effects were due to targeted training, rather than generally increased cognitive engagement or interaction with others. Effect sizes were small for memory, medium for reasoning, and large for the processing speed groups. These gains were retained at each of the subsequent follow-up assessments across 5 years, with 33 percent attrition rate at year 5. Booster sessions had significant effects in the reasoning and processing speed groups. Additional analysis showed that the treatment groups demonstrated smaller decline in their self-reported (but not performance-based) 
daily functioning by the fifth year of the study compared to the control group (Willis et al., 2006).

The results of the 10-year follow-up of the ACTIVE trial showed that the benefits of cognitive training on the trained functions were maintained in the reasoning and processing speed group, but not in the memory group (Rebok et al., 2014). Booster sessions also maintained their effects among the participants, with the exception of the memory training group. Self-reported performance of IADLs in the experimental groups continued to be significantly better than that in the control group at the 10-year followup, although there were no significant group differences on the objective IADL measure. Ten years after cognitive training, 60 to 70 percent of the participants reported the same (or higher) level of IADL performance as 10 years ago, compared to 49 percent of the control group. Forty-four percent of the original sample was retained at year 10 (Rebok et al., 2014). This study confirmed that cognitive training may lead to improved cognitive abilities in healthy older people, with gains maintained up to a decade after the intervention. The fact that the gains were maintained at 10 years in the reasoning and processing speed groups, but not the memory group (which was taught memory strategies) suggests that the benefits of compensatory training may not last as long as the benefits of restorative training. Even though the effect sizes were modest, this is an impressive finding given that the participants were exposed to only 10 or fewer sessions of cognitive training. In addition, the findings revealed that cognitive training may slow the decline in daily functioning and prolong independence, which is the ultimate goal of such interventions (Rebok et al., 2014; Willis et al., 2006). 
Since its publication, several research teams performed secondary data analyses based on the ACTIVE study. For instance, Gross and Rebok (2011) were interested in the participants' utilization of memory strategies on memory tasks. Because it has been established that the use of memory strategies is related to memory performance, the authors analyzed memory test scores among the memory cognitive training group. The sample included 703 memory group participants and 698 control group participants. Initially the memory cognitive training group and the control groups did not differ significantly on their memory and clustering scores. However, higher clustering scores at baseline were related to better recall overall, confirming that memory strategies mediate memory performance. Most importantly, the findings revealed that after the intervention, the participants in the memory group were more likely to use strategies and consequently, had higher memory scores compared to the control group. This increase in strategy use among the memory training group was maintained over 5 years of the study. Also, strategy use was related to improved daily functioning. It can be derived that cognitive training that includes teaching memory strategies may improve older adults' verbal memory and daily functioning (Gross \& Rebok, 2011)

Further, Ball, Edwards, Ross, and McGwin (2010) investigated the relationship between cognitive training and the incidence of motor vehicle collisions among the sample. They selected ACTIVE participants who reported being active drivers at baseline and driving at least 1,000 miles per year. The primary outcome variable was the number of state-reported at-fault motor vehicle collisions per person-mile driven. According to the findings, individuals who underwent processing speed and reasoning cognitive training had a significantly lower rate of at-fault car accidents. This study confirms that 
the benefits of cognitive training are not limited to improved performance on neuropsychological measures and self-reports of daily activities, and that the gains translate into specific areas of functioning such as driving. Driving is not only vital to many older individuals' independence, but may also potentially involve serious risks for both seniors and others on the road if the older adults' cognition is impaired. Thus, undergoing cognitive training may increase both independence and safety among this population and improve road safety at large (Ball et al., 2010).

Among the largest computerized RCTs is a British nation-wide double-blind online study that included 6742 adults older than 50 years, with 2912 older adults over the age of 60 (Corbett et al., 2015). The study compared the effects of a 6-month online cognitive training focusing on reasoning/problem-solving vs. general cognitive training program and active control condition. General cognitive training involved attention, math skills, visuospatial ability, and memory. In both cognitive training groups, difficulty increased as the participants' performance improved. Placebo control group worked on a task that involved putting statements in correct order. The participants were recommended to spend 10 minutes a day on cognitive training or placebo training. Outcome measures were standardized cognitive tests and self-reported IADL performance (Corbett et al., 2015).

After 6 months of cognitive training, participants over the age of 60 in both intervention groups showed greater benefits on IADLs compared to the control group, with small effect sizes. Also, both groups demonstrated gains on measures of reasoning (with small-to-medium effect sizes) and verbal learning (with small effect sizes), and reasoning group exhibited gains on verbal short-term memory and spatial working 
memory, although the effect sizes were very small. Notably, participants in the general cognitive training group performed worse than the control group on a measure of auditory working memory (very small effect size). When the analyses were repeated on a subgroup of participants scoring low on measures of reasoning at baseline, the findings were the same, with the exception of both intervention groups performing worse than the control group on auditory working memory. The findings also confirmed dose-response effect, since more cognitive training sessions were linked with higher effectiveness of cognitive training, particularly reasoning training. The drop-out rate in the study was quite high ( $35 \%$ for participants over the age of $60,88 \%$ overall), suggesting that inperson contact is required to sustain participant interest and motivation (Corbett et al., 2015).

Another large-scale, computer-based RCT was a multi-site double-blind Improvement in Memory with Plasticity-Based Adaptive Cognitive Training (IMPACT) study by Smith and colleagues (2009). The sample was comprised of 487 communitydwelling adults 65 years old and older from California and Minnesota. The participants were randomized into a treatment group and an intensity and frequency-matched educational placebo control group. All participants had computers installed in their homes for the study period. Both groups underwent home-based, self-administered programs, with sessions taking place five times a week for eight weeks ( 40 sessions total). The treatment group underwent training that included exercises focusing on auditory processing speed and accuracy and indirectly involving memory and attention. The computer program was designed to adjust the level of difficulty according to each individual's performance (Smith et al., 2009). 
The drop-out rate in the study was $21 \%$ (Smith et al., 2009). The authors found that compared to the control group, the treatment group demonstrated significantly greater improvements in cognitive functioning, with large effect size on global memory and small effect sizes on other memory measures. Improvements were also seen in the untrained cognitive abilities, suggesting that gains from the cognitive training program may generalize to related abilities. Additionally, the participants in the treatment group had fewer subjective memory complaints (with large effect size), which may imply that the benefits of the training translated into their daily functioning. Due to the inclusion of a placebo control group, the improvements in the intervention group cannot be attributed to increased computer use, duration and frequency of cognitive activity, nonspecific cognitive stimulation, or interaction with staff (Smith et al., 2009).

An RCT based in China (Cheng et al., 2012) compared the effects of cognitive training targeting a single cognitive function and cognitive training involving several cognitive abilities. The participants were 193 healthy adults between the ages of 65 and 75 years. The two intervention groups underwent 24 single-domain or multi-domain cognitive training sessions ( 1 hour-long, 2 sessions per week) over the course of three months. The sessions were conducted in groups. Multi-domain intervention involved memory, reasoning, problem solving, visuospatial and reasoning, as well as handcrafts and physical exercises. Single-domain group received reasoning cognitive training only. Both interventions included a short lecture on health-related issues during the first 15 minutes of each session. Homework was assigned and reviewed once a week. Both treatment groups and the wait-list control group attended a lecture on healthy living every two months (Cheng et al., 2012). 
Assessments took place before and after the intervention, and at 6-month and 12month follow-up interviews (Cheng et al., 2012). Attrition at post-intervention assessment was 10 percent. Six months after treatment, three booster sessions were offered to 60 percent of randomly selected participants in the two intervention groups. Statistical analyses showed that both intervention groups demonstrated overall improvement in their cognitive abilities at post-treatment assessment as compared to the control group with small effect sizes. Both groups showed gains in visual reasoning (with medium effect sizes) and delayed memory (medium effect size for multi-domain group and small effect size for single-domain group). Multi-domain intervention was more likely enhance immediate memory (medium effect size), while single-domain treatment was more likely to improve visuospatial abilities (medium effect size).

At the 6-month follow-up testing, gains in visual reasoning were maintained in both groups and in visuospatial skills for single-domain group. After 12-months, gains in visual reasoning were maintained in multi-domain group participants only, who also exhibited improved delayed memory scores (small-to-medium effect size). At the same time, single-domain group scored higher on a measure of response inhibition (small-tomedium effect size). Booster training had significant effects in both groups and was related to enhanced reasoning, visuospatial skills, and processing speed (Cheng et al., 2012).

The findings point to the benefits of cognitive training in older adults, since intervention groups scored higher than the control group, and attending more sessions was associated with better cognitive outcomes while controlling for baseline scores. Also, it may be derived that multi-domain training is linked with more sustainable gains in 
some cognitive skills in this population. Importantly, the fact that single-domain intervention group improved on a number of untrained functions demonstrates that the specific effects of cognitive training are generalizable to a broad range of abilities. It is important to keep in mind that Cheng and colleagues (2012) chose to incorporate physical activity as part of their two interventions. Previous research has demonstrated a link between physical exercising and cognition, possibly because exercising increases blood flow to the brain, supporting its functioning (Baumgart et al., 2015; Kramer \& Willis, 2002; Plassman et al., 2010). The effects of this factor may be difficult to discern from those of cognitive activities in this study.

An RCT by Shatil (2013) addressed the issue of differentiating between the effects of cognitive training and physical exercise. Shatil (2013) compared three different onsite interventions for cognitive decline: computerized cognitive training, physical exercise program, and combined cognitive and physical exercise training. An active control group was also included and was assigned readings from a book on health in aging. The exclusion criteria were having cognitive or sensorimotor deficits, or taking medications that may affect cognitive functioning. All eligible participants $(N=122 ; 65-$ 93 years old) underwent a computerized assessment developed by CogniFit corporation before and after the intervention. This battery had previously been validated in 861 adults 50-90 years old and cross-validated with standardized neuropsychological measures in younger (but not older) adults (Shatil, 2013).

Cognitive training involved commercially available CogniFit program that administered three tasks out of 21 tasks to participants based on the tasks that they scored lowest on during the baseline assessment (Shatil, 2013). The participants worked on these 
three tasks during the first 20 minutes of each session, and were able to select the other three tasks to work on based on their own preferences for the remaining 20 minutes of each session. Older adults in the cognitive training group were required to attend three group sessions a week for 4 months. The physical activity group attended 45-minute group sessions three times a week for 4 months, while the combined cognitive/physical training group attended both the cognitive and physical exercise programs three times a week each for 4 months. The active control group met once a week for 60 minutes and discussed the assigned reading material. All four conditions allowed for socialization among the participants (Shatil, 2013).

According to Shatil (2013), 48 percent of the participants dropped out of the study. The results indicated that cognitive training group improved significantly on seven CogniFit cognitive battery tasks including those involving divided attention, avoiding distractions, hand-eye coordination, naming, visual processing speed, visual scanning, and global visual memory (effect sizes not provided). In contrast, the cognitive/physical exercise group demonstrated improvement on only three tasks. Moreover, the exerciseonly group and the control groups' performance did not change from the first assessment to the second. No significant 3-way interaction (time X cognitive training X physical exercise) was found. The between-group analysis for the participants who received cognitive training (combined cognitive training only and cognitive/physical training groups) versus the participants without cognitive training (combined physical exercise and the control group) was performed. The findings showed that the two groups who received cognitive training performed significantly better than the two groups who did 
not on eye-hand coordination, global visual memory, processing speed, visual scanning, and naming with medium and large effect sizes (Shatil, 2013).

Even though the between-group analysis for the four arms of the study did not indicate significant group differences, significant effects were found on five of the 14 tests when the four arms were combined into two (those receiving cognitive intervention and those who did not), which provides some support for the efficacy of cognitive training in healthy older population. Importantly, the report indicates that some older adults withdrew due to computer stress, health issues, caregiving for a spouse, and heavy personal activity load (Shatil, 2013). These issues point to barriers to participating in cognitive training among older population, which can be partially addressed by employing home-based and paper-and-pencil approach. Of note, the study utilized CogniFit neuropsychological assessment rather than standardized cognitive measures. Additionally, the author of the study was an employee of CogniFit (Shatil, 2013). Due to these factors, the results should be interpreted with caution.

A relatively small RCT by Mozolic, Long, Morgan, Rawley-Payne, \& Laurienti, (2011) aimed to investigate the effects of inhibition-based training on healthy adults' attention skills. The participants were 66 healthy adults between the ages of 65 and 75 randomized into the treatment and frequency- and duration matched placebo control groups. The treatment group received an 8-week long training (1 session per week) wherein they were taught to suppress irrelevant auditory and visual stimuli. According to Mozolic and colleagues (2011), older adults may be more vulnerable to the distracting stimuli, which may take a toll on their attention and memory, and interfere with their performance on routine tasks. Engaging in exercises that incorporate both relevant and 
irrelevant information resembles the natural distractions in daily tasks and may help older adults minimize the impact of irrelevant stimuli. To ensure that training effects were not attributable to increased computer and keyboard use, all tasks were presented on a screen using a projector, and the participants provided answers verbally or by using a lever. The control group participants attended health education lectures. The participants underwent cognitive testing before and after the treatment, and at 1-month follow-up assessment (Mozolic et al., 2011).

The attrition rate in the study was 6 percent (Mozolic et al., 2011). Following the training, the intervention group participants showed larger improvements in selective visual and auditory attention compared to the control group with small-to-medium effect sizes. These individuals had significantly greater reductions in interference when presented with distractors from outside the modality (e.g., auditory distractors during a visual task, or visual distractors during an auditory task). The authors found that their training had limited effects when it came to distractors from within the same modality. Also, treatment group demonstrated greater improvements in non-trained domains, including processing speed and divided attention (with small-to-medium effect sizes), which points to transfer effects of this intervention (Mozolic et al., 2011).

Another study that focused on training of a specific cognitive domain is an RCT by Rose and colleagues (2015) who evaluated the effects of prospective memory training on cognitive functioning, brain activity during prospective memory tasks (as assessed by event-related potentials on electroencephalogram [EEG]), and IADLs. A total of 58 participants 60-79 years old were included in the study. The intervention group was engaged in prospective memory training computer game for 121 hour-long sessions over 
1 month. Active control group was administered classroom music training with a teacher, and assessment-only control group did not have any intervention sessions (Rose et al., 2015).

Attrition rate was not reported in the article. The participants performed better than the control groups on the trained and untrained prospective memory tasks; however, there were no group differences on other cognitive measures (effect sizes not reported; Rose et al., 2015). The intervention group also performed significantly better on an objective measure of IADLs compared to both control groups. Additionally, EEGs showed significant group differences in the right occipito-parietal cortex, suggesting that the intervention group demonstrated more efficient processing of prospective memory and response regulation, with small effect size. No EEG differences were found in other brain regions. Despite the small sample size, this study is important as it incorporates performance-based assessment of IADLs with the use of daily objects and tasks, as well as brain imaging data (Rose et al., 2015).

An RCT that is of particular interest to the present study is a cognitive training program that utilized home-based, paper-and-pencil tasks (Tranter \& Koutstaal, 2008). The authors combined home-based, self-administered individual training with group creative activities to test their hypothesis that engaging in novel, cognitively stimulating activities has a positive effect on fluid intelligence (reasoning, problem-solving, attention, working memory) among healthy older adults. Forty-four older adults without serious health problems, cognitive impairment, or depression or anxiety symptoms aged 60-75 years were randomly assigned to an experimental or assessment-only control groups. Both groups were told that the study investigated participants' mood rather than 
cognition. The intervention combined home-based, self-administered tasks with onsite group sessions. Specifically, the experimental group was assigned 12 home-based activities, including various picture and word puzzles, visual search, mathematical, and creative drawing activities. The participants were asked to have two sessions per week and work on one activity per session for 40-60 minutes with a two day interval between sessions. The three group on-site sessions involved listening to- and discussing unconventional music, construction of a marble run and a newspaper tower, using origami paper, and playing a board game. The control group participated in several social meetings after the baseline assessment in order to balance the opportunities for socialization for the experimental and control groups. The outcome measures evaluated participants' non-verbal reasoning and visuospatial skills (Tranter \& Koutstaal, 2008).

With regard to attrition, only one participant (in the experimental group) dropped out of the study (Tranter \& Koutstaal, 2008). Additionally, the participants in the intervention group were quite compliant with self-administered home-based activities, as most of them completed all 12 activities except for one or two $(M=11.3)$, which were typically of more artistic nature. At post-intervention assessment, the experimental group scored significantly higher on primary outcome measures with small-to-medium effect sizes compared to the control group. Even though the control group demonstrated slight gains on both measures, these gains were larger for the experimental group. The withingroup analysis showed that the experimental group significantly increased its performance on two of the five outcome measures from pre- to post-intervention assessment. Both groups' mood scores remained unchanged from pre- to postintervention testing (Tranter \& Koutstaal, 2008). 
According to Tranter and Koutstaal (2008), their data support the hypothesis that increased cognitively stimulating activity reduces decline in fluid intelligence and may improve fluid abilities in healthy older individuals. Tranter and Koutstaal's (2008) findings also provide evidence that performing cognitively stimulating tasks is among the protective factors for cognitive decline in healthy older population, as previously found (see reviews by Baumgart et al., 2015; Plassman et al., 2010). An important contribution of the study is that the participants demonstrated improvement on tasks that were not directly trained during the intervention, supporting transfer effects of this multi-faceted intervention to gains on a specific cognitive domain (Tranter \& Koutstaal, 2008).

Relevant to the present study, Tranter and Koutstaal's (2008) results shed light on the feasibility of combined home- and onsite cognitive interventions for older adults. Despite a range of potential logistical and other issues (i.e., participants' understanding, complying with, and accurately reporting study procedures), home-based approach offers a number of important benefits, including reduced participant burden and wider accessibility. Older and frailer seniors may be able to participate, as well as those with health problems and disabilities, lower cognitive status, caregiving responsibilities, and transportation issues. This may also address the self-selection bias, as more cognitively vital older adults tend to participate in laboratory-based studies (e.g., Willis et al., 2006). Also, since the participants tended to omit creative activities, it may be suggested that more concrete and structured cognitive activities should be employed in such interventions. Overall, only one individual out of 44 dropped out of the study, and the participants demonstrated high compliance to self-administered tasks, which points to the 
value of home-based, self-administered paper-and-pencil approach in cognitive training interventions for cognitive decline (Tranter \& Koutstaal, 2008).

In addition to a relatively small sample size, the authors (Tranter \& Koutstaal, 2008) site the short duration of the intervention and lack of follow-up testing as some of the limitations of their research, since 10-12 weeks may be insufficient to demonstrate effects on a rather slow and gradual decline in fluid intelligence. Smaller exposure to social interaction among the control group, inability to discriminate between the effects of individual versus group sessions, and the inclusion of relatively "young" older adults ages 60-65 are also among the limitations of the study. Finally, the study lacked performance-based measures of daily functioning (Tranter \& Koutstaal, 2008).

Summary of findings. Experimental design with randomization in the reviewed RCTs refutes the direction of causality of cognitive engagement critique, according to which cognitively intact older adults tend to enjoy and seek cognitively stimulating tasks and are thus found to be more likely to engage in such tasks (as opposed to stimulating activity contributing to cognitive vitality in older adults; Tranter \& Koutstaal, 2008). Overall, few studies have focused on cognition-based interventions in normally aging older adults. Existing studies evaluated the effectiveness of single-domain (Cheng et al., 2012; Rose et al., 2015; Willis et al., 2006) and multi-domain interventions (Cheng et al., 2012; Corbett et al., 2015; Shatil, 2013) administered individually (Corbett et al., 2015; Mozolic et al., 2009; Shatil, 2013; Rose et al., 2015; Smith et al., 2009), in groups (Cheng et al., 2012; Willis et al., 2006), or in combined individual/group format (Tranter \& Koutstaal, 2008). The setting included onsite programs (Cheng et al., 2012; Mozolic et 
al., 2009; Rose et al., 2015; Shatil, 2013), home-based interventions (Corbett et al., 2015; Smith et al., 2009), or a combination of both (Tranter \& Koutstaal, 2008).

Most of the reviewed RCTs were marked by methodological issues, such as lack of a placebo control group (Willis et al., 2006; Cheng et al., 2012) and small sample size (Mozolic et al., 2011; Rose et al., 2015; Shatil, 2013; Tranter \& Koutstaal, 2008). Only one study included neuroimaging data (Rose et al., 2015), two included a performancebased measure of IADLs (Rose et al., 2015; Willis et al., 2006), and none were replicated. Some studies excluded participants over the age of 75 (Cheng et al., 2012; Mozolic et al., 2011), while others included older adults under the age of 65 (Corbett et al., 2015; Rose et al., 2015; Tranter \& Koutstaal, 2008). Although several studies did not include followup assessments (e.g., Mozolic et al., 2011, Shatil, 2013; Tranter \& Koutstaal, 2008), the data from a large-scale study with 1,2,3,5, and 10-year follow-ups (Rebok et al., 2014; Willis et al., 2006) and a study with a 12-month follow-up (Cheng et al., 2012) did point to maintenance of some of the cognitive training effects in healthy older adults.

Despite the methodological issues, the RCT data are promising and suggest that cognitive training may help maintain cognitive functioning in non-demented older adults. The literature also suggests that booster sessions may improve maintenance of cognitive training effects (Cheng et al., 2012; Rebok et al., 2014), and one study showed that integrating cognitive and physical exercise with psychoeducation may help maintain the gains compared to single-domain cognitive training (Cheng et al., 2012). Importantly, cognitive training may lead to prolonged independence due to improved daily functioning (Rebok et al., 2014; Willis et al., 2006), as well as improved safety resulting from reduced incidence of motor-vehicle collisions (Ball et al., 2010). 
Due to the aforementioned limitations among the studies and variations in dosage, format, and setting of intervention delivery, as well as cognitive functions targeted and assessed, it is difficult to draw conclusions with regard to the "best practices" in this population. It is likely that different approaches are suitable for different populations of older adults, and additional research is needed to identify participant characteristics in relation to specific intervention factors that result in largest post-treatment and follow-up gains on cognition and daily functioning.

Criticisms of cognitive training. Among the main criticisms of cognitive training in healthy older adults is that generally, the effect sizes associated with significant group differences are small-to-medium (Papp, Walsh, \& Snyder, 2009). Self-selection bias may be one of the reasons contributing to this finding. It is possible that those individuals without dementia who are older, have poorer general cognition, and are more frail would be more likely to benefit from cognitive training and demonstrate significant gains in cognition. However, because these individuals are less likely to participate in research and are more likely to withdraw from studies (e.g., Tranter \& Koutstaal, 2008; Willis et al., 2006), current literature might not adequately capture the true potential of cognitive training interventions. Medical conditions, low motivation, and poor treatment adherence may also contribute to only modest gains on cognitive scores in older population (Rebok et al., 2010).

Further, cognitive training for healthy population has been criticized for its limited transfer effects (Kramer \& Willis, 2002). Research participants trained on a specific task showed improvement on that task or a very similar task, but did not improve on other tasks. As a result, it has been argued that cognitive training enhances 
performance on the trained content, but not the underlying cognitive skill, implying that the training may be task-specific or content-specific, but not necessarily process-specific. Thus, the benefits of cognitive training observed in clinical or research settings may not translate into improved daily functioning, which is the main goal of such interventions (Kramer \& Willis, 2002; Slagter et al., 2011).

In response to this criticism, recent studies have incorporated comprehensive neurocognitive assessment as well as measures of daily functioning and quality of life and demonstrated that cognitive training does lead to transfer to untrained functions (Cheng et al., 2012; Corbett et al., 2015; Mozolic et al., 2011; Smith et al., 2009; Tranter \& Koutstaal, 2008), and may lead to improved performance of ADLs (e.g., Corbett et al., 2015; Gross \& Rebok, 2011; Rose et al., 2015; Rebok et al., 2014; Willis et al., 2006). Moreover, cognitive training of working memory was found to improve balance and mobility (walking) in older adults (Smith-Ray et al., 2015), while training of processing speed was linked to maintenance of driving over 5 years (Ross et al., 2015).

In summary, promising results from experimental studies on cognitive training combined with evidence pointing to plasticity of the brain and its seemingly inexhaustible ability to reorganize and learn (Slagter et al., 2011) illustrate that there is a need to continue exploring cognitive training approaches targeting cognitive decline and outcome measures that best capture the effects of interventions. Among the grossly overlooked formats of delivering cognitive training interventions is peer learning.

Peer-based interventions. Programs where peers assist one another in learning or facilitate discussion groups have been extensively researched in educational settings and demonstrated to be beneficial and popular among participants, including older adult 
participants (e.g., Clark, Heller, Rafman, \& Walker, 1997). Additionally, peer support has been linked with improved health behaviors (e.g., Buman et al., 2011) and improved outcomes in mental health and substance use settings (Solomon, 2004). However, little is known about the influence of peers in cognitive interventions, particularly those that involve more than one or two sessions (Margrett \& Willis, 2006).

Collaboration in cognitive training. Among short-term cognitive training studies involving collaboration is a quasi-experiment by Henkel and Rajaram (2011) who evaluated the effects of collaboration on memory, where participants worked on tasks together. The authors were interested in collaboration in normally aging older adults since they may be likely to involve others as a strategy to improve recall. The authors also investigated age differences in relation to collaboration effects and beliefs about collaboration in their study. A group of 96 younger adults (18-27 years old) were compared with a group of 96 older adults (aged 66-92 years) and in each group, half of participants were engaged in collaboration, while the other half were not. The outcome measures included a memory task, which included 72 words from nine different categories. The two most typical words from each category were excluded to examine false recall, and some unrelated words were added for interference effect. After hearing the words, all participants were instructed to write down as many words as they could remember. Next, half of participants were re-tested individually and half were tested in collaboration with two other unfamiliar participants in their age group. All participants were re-tested individually one more time and were also administered a measure assessing collaboration in their daily lives and the factors that they believed influenced collaboration (Henkel \& Rajaram, 2011). 
The results of the study showed that although older adults were able to recall fewer words than younger adults, both groups had a similar pattern of collaborationrelated outcomes (effect sizes not reported; Henkel \& Rajaram, 2011). It was found that both groups demonstrated a comparable degree of collaborative inhibition, which refers to participants recalling less as a group than they would if their individual responses were pooled together. However, collaboration was associated with reduced false recall in both groups. Also, memory gains from collaboration were observed on the last individual retest, although to a smaller degree in the older group. With regard to their self-reports, both groups had positive beliefs about the benefits of collaboration, particularly those individuals who engaged in collaboration during the study. These findings show that collaboration is associated with both costs and benefits for memory, and older adults do not differ significantly in how collaboration affects their memory (Henkel \& Rajaram, 2011). Interestingly, research on collaboration has shown that partner familiarity (stranger vs. partner or spouse) may play a role in how effective collaboration is (Gould, Kurzman, \& Dixon, 1994).

It appears that only one study has evaluated the efficacy of long-term home-based collaborative cognitive training among familiar older adults. In this study (Margrett \& Willis, 2006), 98 participants ( 49 couples) aged 61-89 years ( $M=71$ years) were randomly assigned to one of the three conditions. Thirty participants were included in the individual cognitive training group, 34 in the couples collaborative cognitive training group, and 34 in the no-treatment control group. The three groups did not significantly differ at baseline. Participants in the cognitive training groups received reasoning training based on materials from the ACTIVE study (Willis et al., 2006). The tasks included basic 
reasoning exercises with an emphasis on using strategies, as well as hands-on real-life exercises that involved using maps and bus schedules, completing forms, and other tasks. Sessions lasted between 60 and 75 minutes, where the couples were required to work on tasks individually (individual cognitive training group) or together (collaborative cognitive training group). The participants in the individual training group were encouraged to engage in training at different times than their partner. The participants underwent 10 training sessions, with initial sessions supervised by researchers and some of the sessions videotaped. Assessment included measures of inductive reasoning that required sequencing and identifying patterns (Margrett \& Willis, 2006).

The results showed that both training groups performed significantly better on several tests at post-intervention compared to the control group with medium and large effect sizes (Margrett \& Willis, 2006). However, the two training groups did not differ significantly on any of the measures. The proportion of participants who improved in their scores was also compared across the three groups. It was found that significantly higher proportion of participants in the training groups demonstrated gains on one of the tests. It was concluded that even though the findings supported the effectiveness of cognitive training, the benefits of collaborative versus individual training were not established (Margrett \& Willis, 2006).

The authors (Margrett \& Willis, 2006) believe that their findings confirm that older adults can benefit from self-administered cognitive training. The advantages of such training include the fact that in-home intervention may be more accessible to older adults and that it does not require having a formal trainer, which points to ecological validity of the findings and suggests that the cost of such cognitive intervention would be relatively 
low. In terms of limitations, the authors mentioned that despite their precautions, there was a possibility that the couples in the individual training group discussed the tasks with their spouses, which might have affected the results. Other limitations included lack of follow-up assessment to evaluate maintenance of cognitive gains and lack of measures of functional performance that could determine whether transfer of effects to daily activities has occurred. The authors discussed the potential effects of relationship dynamics on treatment outcomes and recommend including this element in future research (Margrett \& Willis, 2006).

In summary, peer learning with familiar or unfamiliar individuals has not been addressed in the cognitive training literature, with the exception of one study. This study employed collaboration among familiar older adults and showed that collaborative training was no more effective than individual self-administered training (Margrett \& Willis, 2006). It should be noted that when it comes to collaboration where older adults work on cognitive tasks together, participants may not be sufficiently challenged since they share the effort required to work on exercises. For instance, one spouse may compensate for the cognitive weaknesses of the other by doing most of the cognitive work. It may be beneficial to employ coaching rather than collaboration in cognitive training, where older adults administer exercises to one another and check each other's accuracy. It is possible that coaching would eliminate the effects of joint effort or one participant compensating for the other, making it a more challenging and stimulating activity for older adults, which may lead to more dramatic gains in cognitive functioning. However, it seems that coaching has not been previously explored in cognitive training 
literature. Peer-based approaches also raise questions regarding the potential effects of increased social interaction on cognition, as discussed below.

\section{Social Factors and Cognition}

It has been demonstrated that such social variables as marital status and social support may play a role in older adults' cognitive functioning, although the findings are not unequivocal. For instance, longitudinal research shows that being single or living alone is a risk factor for Alzheimer's disease (Fratiglioni, Wang, Ericsson, Maytan, \& Winblad, 2000; Sibley et al., 2002). In addition, in a Netherlands-based study (Van Gelder et al., 2006), older men (70-89 years) who were single, lost their partner, or lived alone over previous 5 years demonstrated a significantly sharper rate of cognitive decline at the 10-year follow-up compared to older men living with a spouse or others.

Conversely, a longitudinal study by Seeman, Lusignolo, Albert, and Berkman, (2001) evaluated 1,189 cognitively intact older adults aged $70-79$ over 7.5 years and revealed that marital status (as well as the number of close ties, number of social groups, instrumental support, the level of conflict/demands, and support provided to others) did not have an effect on the older adults' cognition over time. Instead, higher baseline emotional support predicted better cognitive outcomes among the participants, independent of depression and self-efficacy scores. This suggests that it is not the number of social connections or whether an older adult has a partner/spouse, but the quality of social connections that can positively affect cognition in seniors. Thus, having a supportive partner or close friends may help maintain cognition with age, while a negatively charged relationship or lack of having deep emotional connections may be a risk factor for cognitive decline (Seeman et al., 2001). 
Another longitudinal study (Holtzman et al., 2004) which included 354 adults 50 years old and older $(m=61)$ with a 12-year follow-up, confirmed that emotional support was an independent predictor of higher cognitive functioning. However, in contrast to Seeman and colleagues' (2001) results, the authors found that better cognitive performance was related to more frequent interpersonal activity in larger social networks (Holtzman et al., 2004). It may be suggested that these contrasting results are due to cohort (i.e. age range in study samples) and cultural differences among the studies. Clearly, more research focusing on social variables and cognition in older adults is needed.

\section{Summary and Statement of the Problem}

In summary, age-related cognitive decline is a serious issue as it is related to a wide range of problems on the individual, family, and societal levels (Infurna et al., 2011; Park \& Bischof, 2013). Age-related cognitive decline may affect older adults' driving ability (Kowalski et al., 2011; McKnight \& McKnight, 1999), balance (Herman et al., 2010; Muir et al., 2012), judgment (Boyle et al., 2012), and performance of IADLs (Infurna et al., 2011; Montejo et al., 2012; Tabbarah et al., 2002). As a result, older adults' safety, independence, engagement in leisure and other activities, emotional health, and quality of life may be compromised (Infurna et al., 2011; Calero \& Navarro, 2011; Montejo et al., 2012; Yang \& George, 2005). Age-related cognitive decline may also increase caregiver burden and stress, and increase older adults' need for various services

which takes a toll on the national economy (Infurna et al., 2011; Park \& Bischof, 2013).

Even though it occurs in the majority of older adults, some older adults experience minimal levels of cognitive deterioration (Park et al., 2003), which has been 
linked to lifestyle, health, and social factors, as well as cognitive engagement (Plassman et al., 2010). In the light of its negative effects, there is a need to address age-related cognitive decline in older population based on the up-to-date research on risk and protective factors for cognitive vitality. Maintaining or improving cognitive functions is likely to prolong independence and contribute to well-being in this population and as a result, decrease the personal, caregiver, and societal burdens of age-related cognitive decline.

Research evaluating cognitive training in cognitively healthy older adults has provided some evidence that this non-pharmacological intervention may alter the progression of cognitive decline in seniors, with some studies showing long-lasting effects (e.g., Rebok et al., 2014). These findings highlight the need to develop and evaluate various cognitive training interventions in order to identify best practices in this area. Older adult population is highly diverse and different populations of older adults may benefit from different approaches to cognitive training based on their health and mobility, level of cognition, marital status, familiarity with/access to computer and/or the internet, cultural and residential factors, and personal preferences. It is important to determine which content, format, setting, and dosage of cognitive training are most effective for older adults with certain characteristics.

The majority of existing studies utilized either group, on-site, paper-and-pencil cognitive training or individual, home-based, computerized approach. Group laboratorybased format may create barriers for older adults with mobility, transportation, and scheduling issues, as well as those caregiving. On the other hand, while allowing for flexibility, home-based computerized cognitive training does not incorporate social 
engagement (which has been found to be beneficial for older adults' health, cognition, and well-being) and may not be feasible for those who have limited access to computer or internet, or are uncomfortable using it. There is a lack of cognitive training studies utilizing home-based, paper-and-pencil, peer-based format. Such an intervention may be accessible to broader older adult population as it is characterized by scheduling flexibility and limited facilitator involvement, and minimizes issues related to travel and mobility, caregiving, and computer access or use. Additionally, since none of the previous studies incorporated coaching, there is a need to explore whether this approach is feasible and may enhance the efficacy of cognitive training.

\section{Present Study}

Purpose. The purpose of the study was to address current gaps in the literature and evaluate feasibility, participant acceptability, and efficacy of a novel, home-based dyadic intervention with coaching in community-dwelling older adults without cognitive impairment. The participants' pre- and post-intervention scores were used to evaluate changes in cognition and relationships among cognitive, self-report, and demographic variables. Semi-structured interviews were utilized to explore the participants' experiences with the intervention.

Conceptual framework. Even though research supporting the link between cognitive training and neurological changes is at its infancy (Park \& Bischof, 2013), emerging imaging data combined with existing cross-sectional and longitudinal studies on risk and protective factors for age-related cognitive decline support the validity of the STAC theory and the concept of neuroplasticity. Based on this literature, it was hypothesized that through the mechanisms of neuroplasticity and scaffolding, cognitive 
training of verbal memory, language, reasoning, and visual processing would promote activation of existing and alternative neuronal circuitry and lead to maintenance or gains in these cognitive functions. In addition, it was expected that transfer of training effects to untrained functions would occur, as demonstrated by gains on visual memory, delayed memory, or response inhibition. It was expected that improved cognition, particularly executive functions would also lead to improved performance of IADLs, since these functions are believed to be at the core of individuals' daily activities (e.g., Royall et al., 2007).

Further, it was expected that in its turn, improvement in cognition and IADLs would translate into enhanced quality of life among the participants. Moreover, it was hypothesized that the social engagement and coaching component that is unique to the present intervention would further enhance cognition, daily functioning, and quality of life. Drawing on the Life Course Perspective discussed previously, it was hypothesized that social engagement would contribute to an enriched environment for participants, which may improve bio-psycho-social outcomes. The conceptual framework of the study is presented in Figure 1 below. 
Figure 1.

\section{Conceptual Framework.}

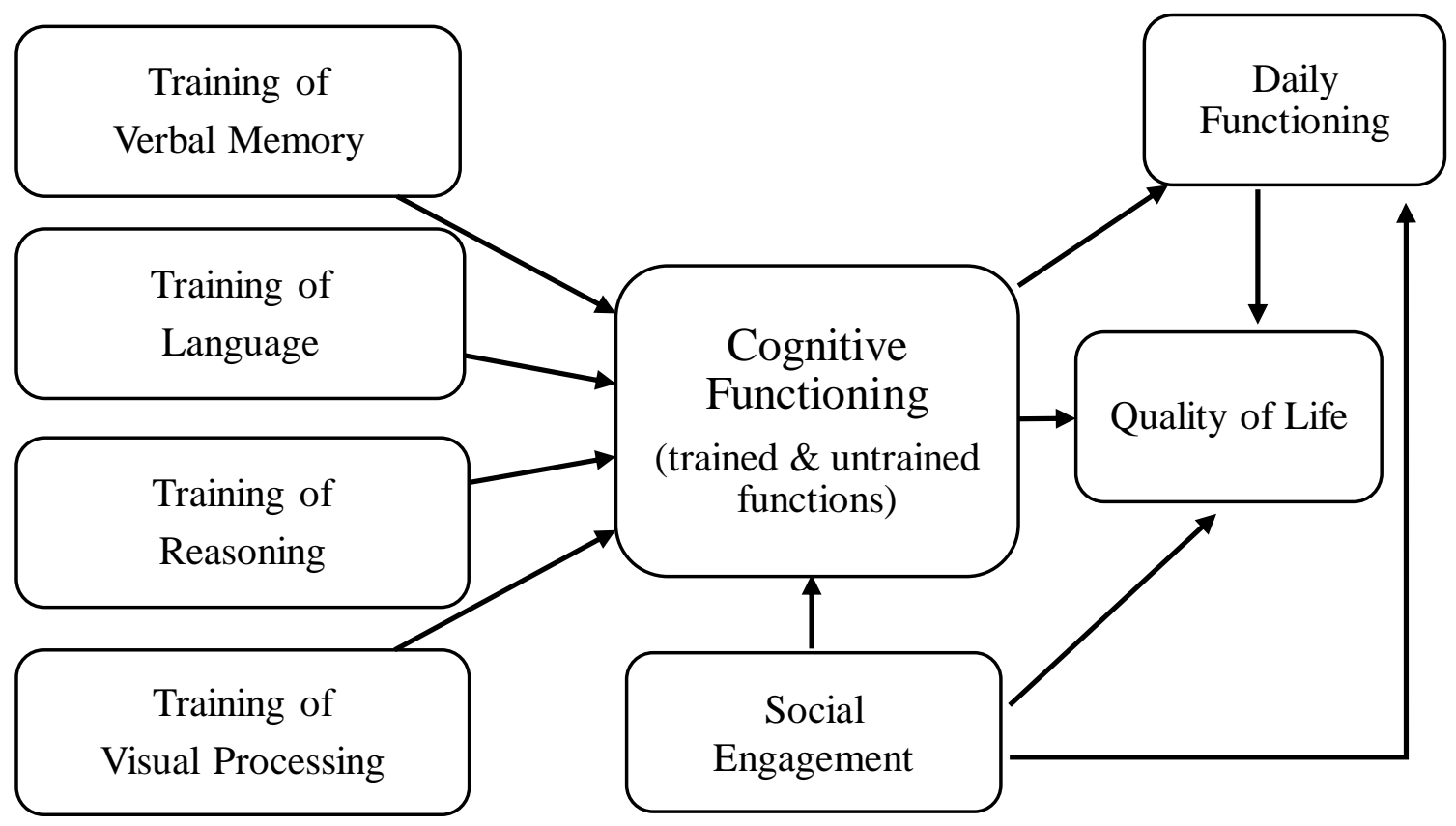

Note. The figure illustrates that cognitive training of verbal memory, language, reasoning, and visual processing, combined with purposeful social engagement leads to improvement in overall cognitive functioning, including untrained cognitive skills. This improvement in cognition, together with social engagement lead to improved daily functioning and quality of life. In addition, enhanced daily functioning also contributes to improved quality of life.

Practical and economic reasons. The practical reasons for the study are related to the unique format of intervention. Cognitive training program developed for this study is highly flexible as it is self-administered at home without the use of a computer and thus, it addresses the potential issues that may arise with onsite programs such as problems with physical mobility, scheduling, space availability, weather, transportation, or computer use. As an example, in the ACTIVE trial (Rebok et al., 2014; Willis et al., 
2006), the participants that withdrew from the study after randomization or during the intervention period were more likely to be older, single, have health issues, and lower initial cognition scores. Further, in addition to health problems and having to care for a spouse, computer stress was among the reasons for attrition in a computerized cognitive training study (Shatil, 2013). This illustrates that on-site and/or computerized interventions may be vulnerable to self-selection bias because older adults who complete the training tend to be younger, healthier, more cognitively intact, and more computersavvy. However, the participants that may be likely to benefit from the intervention and may show more dramatic gains on cognitive measures may be the ones who tend to withdraw, which can result in smaller effect sizes in these studies. Some scholars (e.g., Taussig \& Pontón, 1996) argue that it is important to include older adults with non-acute chronic illnesses in cognition research because "they are representative of the real world" (p.51). For this reason, including older adults over the age of 65 with a variety of functional and non-acute medical issues in the study sample, as opposed to younger and more vital seniors, possibly contributed to the ecological validity of the intervention. Similarly, the format of the intervention likely addressed many barriers associated with onsite and computerized interventions, and thus, reduced self-selection bias.

In terms of economic reasons, cognitive training in the present study probably requires fewer resources compared to onsite cognitive training programs, which can be costly (Rebok et al., 2010). In the dyadic intervention, the burden on agencies in the community is reduced since it requires limited supervision and eliminates the need for facilities or transportation services. From the macro-level perspective, the study contributes to the existing knowledge in aging and cognition that may inform policy with 
regard to community-based services promoting health and cognitive vitality for healthy older adults. Specifically, the study highlights the benefits of home-based cognitive interventions, which may be included among the services offered by senior centers, health centers, and other agencies in the future. Cognitive training interventions for cognitive decline may be identified as services covered by the Older Americans Act, which emphasizes the importance of preventative services and evidence-based interventions (Administration on Aging, 2008). Ultimately, the study may help prolong independence and enhance quality of life among the growing aging population, as well as their caregivers. This may lead to decreased personal, family, and societal burdens associated with natural cognitive decline.

Research questions and hypotheses. The purpose of the mixed-methods study was to evaluate feasibility and participant acceptability of a home-based, selfadministered dyadic cognitive training with coaching. The research questions and hypotheses were as follows:

Research Question 1. What are the participants' retention and adherence rates? Hypothesis 1.1. At least 80 percent of participants will remain in the study at postintervention assessment (estimate based on existing cognitive training studies with retention rates of $2-48$ percent).

Hypothesis 1.2. The participants will complete at least 80 percent of sessions (19 out of 24 sessions).

Research Question 2. What are the participants' experiences with the intervention? (Hypotheses not applicable; research question to be explored through qualitative interviews). 
Research Question 3. How will the participants' scores on cognitive and self-report measures change from baseline to post-intervention assessment?

Hypothesis 3.1. The participants' will not decline in their cognitive and self-report scores from pre- to post-intervention. Improvements in some cognitive skills may be observed (not possible to predict which cognitive skills may improve due to variability of results in the literature).

Research Question 4. Is there a relationship between participant characteristics and their scores on the pre- and post-intervention cognitive measures?

Hypothesis 4.1. Lower education, older age, and lower health status will be related to lower baseline cognitive scores, while gender will have no effect on baseline cognitive scores. 


\section{METHODS}

\section{Research design}

In order to evaluate feasibility of the novel, dyadic cognitive training intervention in the target population and answer the research questions, a mixed methods quasiexperimental design was employed. Simple percentage calculations were used to evaluate Research Question 1 and assess the participants' retention and adherence rates. Qualitative strategy was used to address Research Question 2 and explore the participants' experience with the intervention. Specifically, in-depth, semi-structured interviews were administered to all intervention group participants in order to evaluate adequacy of the materials and participant acceptability of the intervention, and identify ways to improve "user-friendliness" of the manual and intervention. Lastly, quasiexperimental, quantitative approach was employed to address Research Questions 3 and 4 and compare pre- and post- intervention scores for preliminary assessment of efficacy of the intervention and to evaluate the role of demographic variables.

The procedures of the study align with Stage I of the Stage Model of Behavioral Therapy Research, which mirrors the stage model of pharmacological and medical research (Rounsaville \& Carroll, 2001). In Stage I of this model, "manual development, therapist training, assessment of therapist adherence, and competence and feasibility testing are research tasks that are guided by scientific standards" (p. 134). Stage I seeks to establish elements required for subsequent research activities. It lays the foundation for Stage II of the model, efficacy testing, and allows fine-tuning various components of the intervention and addressing any issues before proceeding with large and costly RCTs (Rounsaville \& Carroll, 2001). Double-blind, placebo-controlled RCT is considered to be 
a gold standard in medicine and science research, as it minimizes human bias and maximizes the validity of results (Kaptchuk, 2001). However, Stage I research is a necessary step as it guides the development of RCTs.

Because Stage II research focuses on internal validity of an intervention, such research standards as sufficiently large sample, stringent inclusion and exclusion criteria, randomization, and follow-up assessment are of utmost importance. In contrast, in Stage I studies, exploratory and descriptive aspects of research and external validity of the intervention take precedence, while internal validity is given lower priority (Rounsaville \& Carroll, 2001). For these reasons, the feasibility study included a small sample of older adults using relatively broad inclusion criteria, and did not employ a control group. The primary goal of the study was to evaluate feasibility of the novel intervention and lay foundation for future confirmatory and efficacy trials. In the study, the participants underwent brief cognitive assessment followed by home-based dyadic intervention up to 24 weeks-long. After the conclusion of the intervention, the participants were re-assessed and interviewed. The goal was to determine whether the novel intervention was relevant for the target population and to identify changes that would increase acceptability and adherence for this format of cognitive training.

\section{Sample}

Population. The population of interest is older adults without MCI or dementia in the US.

Participants. The participants were community-dwelling English-speaking older adults without MCI or dementia who resided in Miami-Dade County in Florida. 
Eligibility. According to Mody and colleagues (2008), older individuals are oftentimes under-represented in clinical research due to modifiable and non-modifiable barriers associated with participation in studies. The authors recommend that scholars utilize less stringent inclusion and exclusion criteria when it comes to older adults in order to maximize the sample's representativeness of the target population. In the present study, the decision was made not to limit the dyads to married couples (as in Margrett \& Willis, 2006 study) since females tend to outlive males, which would potentially exclude many female participants. The dyads were expanded to family members, relatives, friends, or neighbors, as long as they had no difficulty meeting on the regular basis (i.e. transportation issues or space limitations). The inclusion and exclusion criteria were as follows:

\section{Inclusion criteria.}

a) Aged 65 years or older

b) English-speaking

c) Have adequate English reading skills (see Measures section below).

d) Live independently in the community.

e) Interested in participating in the study.

f) Available for the duration of the study.

g) Able to meet with partner twice a week for 12 weeks.

h) Have space adequate for assessment and cognitive training (e.g., desk or table with at least two chairs, minimal distractions). 


\section{Exclusion criteria.}

a) History of Mild Cognitive Impairment, Alzheimer's disease, Parkinson's disease, Multiple Sclerosis, or other conditions that cause dementia or cognitive impairment.

b) History of acute medical, neurological, or psychiatric condition such as stroke, cancer, or substance abuse within the previous year that is not medically managed or in remission.

c) History of moderate or severe traumatic brain injury, brain surgery, or other conditions that may affect cognitive functioning (based on the screening interview).

d) Symptoms of moderate or severe depression (see Measures section below).

e) Significant sensory-motor deficits or functional limitations that may impair hearing, ability to read and write, or ability to sit at a table (based on the screening interview).

f) Evidence of significant cognitive deficits as determined by extremely low scores on measures of global cognitive status and specific cognitive functions (see Measures section below).

g) Previous participation in a research study or a community or clinic-based program involving cognitive training.

h) Participation in a research study or clinical evaluation that included neuropsychological assessment within previous 2 years.

Sample size. Power analysis was performed using G-Power (Faul, Erdfelder, Lang, \& Buchner, 2007) to estimate the number of participants required to address Research Question 4 (change in the participants' scores on the outcome measures from pre- to post-intervention). According to the power analysis for T-Test family, same subject design, 19 participants are needed in order to achieve power of $80 \%$ with medium 
effect size of 0.7 and significance level set at 0.05 (two-tailed). Since an even number of participants are required for the dyadic format of the study, it was estimated that 20 older adults (10 dyads) would be needed to evaluate Research Question 3 (differences from pre- to post-intervention assessment).

\section{Tests and Measurements}

The participants underwent pre- and post-intervention assessment. Information with regard to validity and reliability of the measures employed in the study is provided, where available. It is recommended that researchers include limited number of measures of relatively short duration when dealing with older adults in order to reduce fatigue and frustration and improve recruitment and retention rates of older participants (Mody et al., 2008). Thus, assessment included only those measures that were necessary for screening and addressing the research questions in the study. Where applicable, permissions to use the measures are provided in Appendix 1. The description of screening, cognitive, and self-report measures are described below.

\section{Screening measures.}

Slosson Oral Reading Test- Revised. Because the dyadic intervention is selfadministered and relies heavily on the participants' reading ability, it was important to include a measure of English language proficiency in the study. Slosson Oral Reading Test Revised, $3^{\text {rd }}$ Edition (SORT-R3; Slosson \& Nicholson, 2002, Appendix 2) was administered as part of the screening process to ensure that the participants were able to read and understand training materials and instructions. This measure includes 200 words arranged in groups of 20 words of increasing difficulty and takes 3-5 minutes to administer. The age and grade-equivalent norms are based on a nationally representative 
sample of children and adults, with an additional category for Hispanic-Americans, Asian-Americans, Pacific Islanders, and other ethnic groups whose primary language is English. Concurrent validity of SORT-R3 with achievement tests is reported as .90 and higher (Slosson \& Nicholson, 2002). Individuals were required to have the reading level of at least $8^{\text {th }}$ Grade based on the norms included in the scoring manual. Permission to use this measure was provided by Slosson Educational Publications via personal email communication (see Appendix 1).

Mini-Mental State Examination. Mini-Mental State Examination (MMSE; Folstein, Folstein, \& McHugh, 1975; Appendix 3) was used to evaluate individuals' global cognitive status and exclude participants who potentially have pathological forms of cognitive decline such as MCI or dementia. It is a brief measure widely used in research and includes items of orientation to time and place, memory, and visual and auditory information processing. According to Mitrushina \& Satz (1991), the reliability of MMSE is .45-.50, while the validity is not reported and correlations with similar measures are described as high. It has become a common practice in cognitive training studies to include MMSE (Papp et al., 2009), with cut off scores ranging from 22 to 26 (e.g., Willis et al., 2006, Smith et al., 2009). Based on these studies, the decision was made to use the middle value of 24 as the cut-off score. Thus, in order to qualify for the study, individuals had to have an MMSE score of 24 or above. MMSE is provided free of charge by The Hartford Institute for Geriatric Nursing, Division of Nursing, New York University and can be used for not-for-profit educational purposes without a written permission or license (see www.hartfordign.org). 
Patient Health Questionnaire (depression). A measure of depression was included since depression has been found to be related to cognition in older adults (Kohler et al., 2010; Plassman et al., 2010). Depression was assessed by the Patient Health Questionnaire (PHQ-9; Kroenke \& Spitzer, 2002; Appendix 4). This instrument includes 9 items asking how many days a participant felt a certain way over the past two weeks, and one question assessing difficulty dealing with any of the identified issues in daily life. Such domains as loss of interest, sadness, sleep and appetite disturbances, psychomotor changes, fatigue, and suicidal ideation are included. The scores range from zero to 27 , with higher scores indicating higher level of depression. The $10^{\text {th }}$ question is not included in the final score calculation. According to the instrument manual (Kroenke \& Spitzer, 2002), the scores of 15 or above point to moderately severe- to severe depression. Thus, the score of 15 was the cut-off score in the study. Reliability for this instrument is reportedly $.86-.89$, with sensitivity of $88 \%$ and specificity of $88 \%$ for major depression. Test-retest reliability and construct validity are reported as excellent, and correlation between self- and examiner-administered surveys is .84 (Kroenke, Spitzer, \& Williams, 2001). PHQ-9 is available in the public domain and does not require a permission to be reproduced and used (see https://phqscreeners.pfizer.edrupalgardens. com/sites/g/files/g10016261/f/201412/instructions.pdf).

Cognitive measures. The following cognitive tests are widely used in research and practice settings and have been employed in previous studies on cognitive training in older adults (e.g., Cheng et al., 2012; Mozolic et al., 2011; Rose et al., 2015; Smith et al., 2009). 
Stroop Color and Word Test. The Stroop Color and Word Test includes three trials and assesses cognitive flexibility/inhibition, in addition to attention and processing speed (Golden \& Freshwater, 2002; Appendix 5). In the first trial, Word Reading, the participant is instructed to read a list of simple words (red, blue, and green) printed in black ink on a sheet of paper as fast as possible. In the second trial, Color Naming, the participant is instructed to name the color of the ink in a list of "XXXX"s printed in different colors as fast as possible. In the third trial, Color-Word, a list of the words including "red," "green," and "blue" is presented on a page, but each word is printed in ink different from the color that it describes. The examinee is required to name the color of the ink for each word. Each trial has a 45 -second time limit, and the measure takes several minutes to administer. The reliability of Stroop Color-Word Test is reportedly between .83 and .91 , and validity is indicated as high (Spreen \& Strauss, 1998). Permission to use Stroop test was provided via personal email communication by Dr. Katherine Genseke, Product Manager for Psychological and Special Education Materials, Stoelting (see Appendix 1).

Trail Making Test. The Trail Making Test (TMT) from the Halstead Reitan Neuropsychological Battery (Reitan \& Wolfson, 1993; Appendix 6) is another measure of executive functioning that also requires the use of visuomotor ability with eye-hand coordination. It assesses visual attention, processing speed, sequencing, and working memory/cognitive flexibility. In Part A (TMT A), participants are presented with a sheet of paper that has digits printed in a random arrangement. They are asked to use a pencil and connect digits in ascending order with straight lines as fast as they can. In Part B (TMT B), both digits and letters are printed randomly on a page, and the individuals are requested to do the same while alternating between digits in ascending order and the 
letters in alphabetical order. TMT typically takes several minutes to administer. The reliability of TMT-A is .79 and TMT-B is .89 (Reitan \& Wolfson, 1993). Permission to use TMT (with qualifying purchase) was provided by the publisher, The Neuropsychology Center, via personal email communication (see Appendix 1).

Repeatable Battery for the Assessment of Neuropsychological Status- Update. Repeatable Battery for the Assessment of Neuropsychological Status- Update (RBANSU; Randolph, 2012; Appendix 7) is used to evaluate core cognitive functions in individuals 12-90 years of age. It is comparable to its original version (Randolph, 1998) and includes 12 subtests that take about 30 minutes to administer. The subtests assess five domains: Immediate memory (List Learning and Story Memory subtests), Visuospatial/Constructional (Figure Copy and Line orientation subtests), Language (Picture Naming and Semantic Fluency subtests), Attention (Digit Span and Coding subtests), and Delayed Memory (List Recall, List Recognition, Story Memory, and Figure Recall subtests). RBANS-U produces standardized subtest and domain scores, as well as the Total cognitive functioning score. This measure includes four alternate equivalent forms, and the participants in the study were administered two different forms at pre- and post-intervention assessment in order to address potential practice effects. Based on a sample of 631 older adults without neurological conditions, Cronbach's alpha for RBANS indices is .86 , with intercorrelations ranging from .25 to .79 (Gontkovsky, Beatty, \& Mold, 2004). Test-retest reliability between RBANS forms A and B (test-retest interval 1-134 days) was reported as .77 for healthy adults $(\mathrm{N}=99)$ and .84 for 181 patients with schizophrenia (Wilk et al., 2002). Permission to use RBANS-U was 
provided by the publisher, Pearson Assessments (San Antonio, TX) via personal email communication (see Appendix 1).

A summary of cognitive measures and the functions that they assess is presented in Table 1.

Table 1.

Description of Cognitive Functions and Corresponding Measures.

\begin{tabular}{|c|c|}
\hline Cognitive Domain & Measure (Cognitive Function) \\
\hline \multirow[t]{5}{*}{ Verbal Memory } & RBANS-U List Learning (immediate memory) \\
\hline & RBANS-U Story Memory (episodic/narrative memory, immediate) \\
\hline & RBANS-U List Recall (delayed memory) \\
\hline & RBANS-U List Recognition (delayed memory) \\
\hline & RBANS-U Story Recall (episodic/narrative memory, delayed) \\
\hline \multirow[t]{2}{*}{ Language } & RBANS-U Picture Naming (long-term recall, general knowledge) \\
\hline & RBANS-U Semantic Fluency (long-term recall, general knowledge) \\
\hline Visual Memory & RBANS-U Figure Recall (figural memory, delayed, visuomotor) \\
\hline \multirow[t]{2}{*}{ Visuospatial Skills } & RBANS-U Figure Copy (visuomotor) \\
\hline & RBANS-U Line Orientation (visuospatial reasoning) \\
\hline Executive Functions & RBANS-U Digit Span (attention, working memory, auditory) \\
\hline (Information processing, & RBANS-U Coding (attention, working memory, visuomotor) \\
\hline Attention, & Stroop Word (processing speed, verbal) \\
\hline Working Memory, & Stroop Color (processing speed, visual) \\
\hline \multirow[t]{3}{*}{ Cognitive Flexibility) } & Stroop Color-Word (multitasking, response inhibition, visual) \\
\hline & Trail Making Test A (attention, visuomotor) \\
\hline & Trail Making Test B (attention, sequencing, multitasking, visuomotor) \\
\hline
\end{tabular}




\section{Self-report measures.}

World Health Organization Quality of Life-Older Adult. Quality of life was evaluated using a measure developed by the World Health Organization (WHO, 2006) for older adults, WHOQOL-OLD (Appendix 8). This measure consists of 24 items that assess six facets including Sensory Abilities, Autonomy, Activities, Social Participation, Death and Dying, and Intimacy. The measure also provides the Total quality of life score. Internal consistency is reported as .72-.88 for facets and .89 for the Total score (WHO, 2006). A copy of WHOQOL-OLD and the permission to use it was provided by Donald Bushnell, Associate Director, Health Research Associates, Inc. (see Appendix 1).

Research and Development 36-Item Short-Form Health Survey. Research and Development (RAND) 36-Item Short-Form Health Survey (RAND SF-36; Ware \& Sherbourne, 1992; Appendix 9) was used to evaluate overall health. It includes 36 items related to different aspects of individuals' health. The results provide eight scaled scores (Physical Functioning, Role Limitations due to Physical Health, Role Limitations due to Emotional Problems, Energy/Fatigue, Emotional Well-Being, Social Functioning, Pain, and General Health). Reliability for RAND SF-36 scales is reported as .78 - .93. This measure is available in the public domain free of charge (http:/www.rand.org/health/ surveys_tools/mos/mos_core_36item_survey.html), and the authors state that no written permission or license is required to use the instrument (see http://www.rand.org/health/ surveys_tools/mos/mos_core_36item_terms.html).

Bayer Activities of Daily Living. Bayer Activities of Daily Living (B-ADL; Hindmarch, Lehfeld, de Jongh, \& Erzigkeit, 1998; Appendix 10) is a caregiver report that includes 25 items assessing individuals' ability to perform daily tasks and engage in self- 
care and IADLs. Its internal validity is reported as .98 (Choi et al., 2003; Folquitto et al., 2007). B-ADL also showed high test-retest reliability (Choi et al., 2003) and was found to effectively discriminate between healthy older adults, those with mild dementia, and those with moderate dementia (Folquitto et al., 2007). A copy of B-ADL and the permission to use it was provided by one of the authors, Dr. Hartmut Lehfeld (Nürnberg, Germany) via personal email communication (see Appendix 1). Although this measure was designed as an informant report, it can also be administered as a self-report measure of IADL, which has been the case in some studies (e.g., Pusswald et al., 2015). All participants were administered the measure as a self-report, while the spousal dyads were administered both the self-report and caregiver report pertaining to their spouse.

\section{Intervention}

Setting, frequency, and duration. Cognitive training intervention was designed to be self-administered at home, but other locations convenient for participants were also acceptable. The key feature of cognitive training was coaching and turn-taking, where the participants took turns training one another for 30 minutes each during every 1 hour-long session. The older adults did not collaborate on tasks; rather, they acted as facilitators of cognitive training for one another and checked each other's accuracy, which was also expected to contribute to the cognitive stimulation in the intervention. The intervention was designed to take place twice a week for 12 weeks, with a total of 24 sessions. The days and times of sessions were based on participants' schedules and preferences.

Materials. Each pair received a shopping bag with a 30-minute hourglass sand clock, writing utensils, and the Brain Training manual. 
Manual. The Brain Training manual was developed for the study and has not been previously used. The pages in the manual were printed in color on 3-hole punched paper and divided into two large binders (287 and 218 pages each, approximately 350 tasks total). The manual was designed to be shared by each dyad, so that the participant who is the trainer first had the manual for 30 minutes, and then passed the manual to his/her study partner who then became the trainer. During each session, the dyad continued where they previously finished. After switching roles, the trainer continued with the next task in the manual, as opposed to going back and repeated the tasks. Thus, none of the tasks were repeated among the participants in the dyad and older adults were presented with unique exercises during each session.

The manual began with a Log Sheet and a description of how to use the manual (Appendix 11), followed by the tasks. The Log Sheet included information for each session, such as who was the trainer first, session date and time, and pages in the manual completed during the session for each person.

Sources. The tasks were selected from two sources, Workbook of Activities for Language and Cognition (WALC; Tomlin, 2002) and Visual Processing module from the Brainwave-R series (Malia, Bewick, Raymond, \& Bennett, 2002). Permissions to use these sources were obtained from publishers via personal email communication (see Appendix 12).

1) WALC-2: Cognitive Rehab book from the WALC series (Tomlin, 2002) is used to improve neurocognitive functioning in individuals over 14 years of age who have suffered a stroke, head injury, or other neurological impairment. This workbook contains exercises of varying difficulty targeting different neurocognitive deficits, including those 
in attention/ concentration, processing speed, and abstract reasoning, among others. Even though it was developed for clinical populations, the tasks of higher difficulty level can be applied to healthy older adult population.

In terms of prior research, it appears that this resource has not been previously used in intervention studies focusing on healthy older adults. However, a recently published feasibility and efficacy study on cognitive training for patients with Alzheimer's disease (Kanaan et al., 2014) used exercises from WALC Cognitive Rehab (Tomlin, 2002), among other workbooks by the same author. In this study, a combined computerized and paper-and-pencil cognitive training program was evaluated in a sample of 21 adults with early stages of Alzheimer's disease. The program targeted attention and memory and was highly intensive, with 4-5 hours of cognitive training per day, five times a week over a 2-week period. The training took place onsite and was administered individually. Outcome measures included standardized measures of logical memory, verbal fluency, and executive functioning, as well as the directly trained computer-based tasks. Assessment took place before and after the training and at 2-month and 4-month follow-ups (Kanaan et al., 2014).

The results confirmed feasibility of the study, as only one participant had to reschedule two of the training days (Kanaan et al., 2014). With regard to cognition scores, absence of decline or improvement was seen on all standardized measures at postintervention and follow-up assessments, with the exception of one of the two verbal fluency measures (category fluency), which declined. Effect sizes were not provided in the published report. Although this study utilized tasks from WALC Cognitive Rehab and 
other resources, detailed information about the specific use of WALC was not available in the article (Kanaan et al., 2014).

2) Brainwave-R series: Cognitive Strategies and Techniques for Brain Injury Rehabilitation (Malia et al., 2002) include numerous paper-and-pencil exercises for individuals who sustained head injury, but are also suitable for healthy older population. This program contains exercises of varying difficulty targeting different cognitive domains. In the present study, only the Visual Processing module was used.

Based on the literature search, Visual Processing module from Brainwave-R also has not been employed in healthy older adults. However, exercises from several Brainwave- $\mathrm{R}$ modules were adapted for use in a Japanese feasibility study (YamamotoMitani, Matsuoka, \& Fujii, 2007) on home-based cognitive rehabilitation for older adults with cognitive impairment due to traumatic brain injury or subarachnoid hemorrhage, or those attending memory clinics. The workbooks from Brainwave-R series were selected individually based on each participant's deficits. Nine participants and their families received training on how to use the workbooks and were instructed to engage in cognitive rehabilitation 30-60 minutes five days a week for eight months (Yamamoto-Mitani et al., 2007).

Six of the nine older adults completed the study, with age ranging from 59 to 78 years (Yamamoto-Mitani et al., 2007). Research staff provided assistance and support via home and clinic visits, as needed. Neuropsychological tests were administered before and after the intervention, and four months into intervention. The results showed that the participants did not decline on measures of executive function and everyday memory, and demonstrated improvement on measures of attention (Yamamoto-Mitani et al., 2007). It 
appears that the only other study that utilized Brainwave-R series evaluated cognitive rehabilitation in patients with schizophrenia and used Executive Functioning module of the Brainwave-R (i.e., Davalos, Green, \& Rial, 2002).

Task adaptation. The Brainwave-R (Malia et al., 2002) and WALC (Tomlin, 2002) workbooks were originally designed to be administered by a trained professional, so the tasks were adapted for use by older adults in the dyadic, self-administered format. The selected tasks from these sources were scanned or retyped into a PowerPoint document. Some exercises were divided into several tasks in order to shorten them and increase task variety during each session. Instructions were adapted or created for dyadic use and placed at the top of each task. Every task began on a new page. Different colors were also used to make the manual user-friendly. Each task began with instructions for the trainer in gray, followed by the instructions that the trainer was to read to his/her study partner, typed in blue. Correct answers were added for certain tasks in green, so that the participants could check each other's accuracy or give each other hints or clues. Finally, the tasks were arranged to alternate the targeted cognitive domains, as well as verbal vs. visual content in order to avoid redundancy. The tasks were also arranged to increase in difficulty throughout the two binders.

Some exercises required the trainer to read the task out loud and ask questions, while others required the trainer to give the labeled Worksheet to his/her study partner to work on. In such cases, the worksheet always followed the task and the trainer was instructed to tear the Worksheet page out of the binder and give it to his/her study partner. The participants were requested to keep the used worksheets in the back of the manual. 
Functions targeted. The intervention targeted verbal memory, language, reasoning, and visual processing. Importantly, none of the intervention tasks were the same as the measures included in the cognitive assessment.

1) Verbal memory. Verbal memory exercises included tasks of verbal working memory, where the participants were required to listen to several items (numbers or words) and manipulate that information by naming the largest number, reversing the order of items, or naming the word that did not belong with the others. The tasks also targeted episodic verbal memory, as the participants were instructed to listen to a short story or a paragraph on general knowledge and then answer questions about that story or paragraph. Long term memory was trained by participants having to recall several US presidents, wars, or famous actors. Examples of verbal memory tasks are provided in Appendix 13.

2) Language. Language training focused on verbal comprehension (following directions, repeating statements, etc.) and verbal fluency (telling different meanings of the same word, such as "mark," "clip," "bat," etc., naming a word from a specific category starting with a certain letter, etc.). The participants were also required to identify words with missing letters and unscramble sentences by putting words in correct order. See Appendix 14 for examples of language tasks.

3) Reasoning. An example of a reasoning task was listening to clues about different characters and then filling out a table about the characters using deduction and elimination. Other examples were tasks that involved arranging steps of a process (such as cooking) in the correct order, thinking of ways to improve something, completing analogies, and guessing what the key word is based on clues. Also, the participants were 
required to imagine the same situations from the perspective of different characters and identify differences and similarities among two similar objects. See Appendix 15 for examples.

4) Visual processing. Visual processing tasks focused on visual processing speed and visual attention and scanning, such as searching for a letter or a number among lines of mixed letters/numbers, searching for letters among a string of letters that also appear in a key word, decoding a message represented by flag signals, or finding matching shapes. See Appendix 16 for examples of visual processing tasks.

\section{Procedure}

Institutional review. The study was submitted for a full review to the Florida International University Institutional Review Board (IRB) and was approved. The researcher and the principal investigator successfully completed the Collaborative Institutional Training Initiative (CITI) Program course on protection of human subjects (CITI Program, 2015).

Expert panel. The expert panel included three neuropsychologists with doctoral degrees in psychology (Ph.D./Psy.D.) who each had 5-15 years of practicing experience in neuropsychology, including cognitive assessment and cognitive training. The panel reviewed the Brain Training manual in detail and provided feedback on how the manual could be improved. The comments and suggestions included clarifying the instructions and formatting, changing the content, and providing correct answers. The manual was modified based on the expert panel's feedback.

Test trial. An older married couple (both 60 years old) whose first language was not English participated in the test trial of the manual. The session simulated Session 1 of 
the intervention, the only cognitive training session facilitated by the researcher. The couple was instructed on how to use the Log Sheet and administer the tasks to one another. The couple was encouraged to mention anything that was not clear or confusing. The participants had several comments regarding the instructions in the manual, which were modified accordingly. The test trial also provided information regarding the approximate number of tasks needed for each session.

Participant recruitment. Participant recruitment took place between May 2015 and October 2015. Recruitment took longer than expected due to seasonal and locationrelated factors: many individuals travel during the summer months when recruitment took place or are "snowbirds" (live in the North-East during the summer and come back to Florida during the colder months). Additionally, many older adults in Miami area are Spanish-speaking and are not fluent in English.

IRB- approved flyers (Appendix 17) with information about the study and researcher contact information were placed in Miami, FL area public libraries, low income senior housing buildings, community and health centers, and senior centers (with permissions from administrators). The study was also advertised on Miami-Dade Craigslist website, in the "Events" and "Volunteers" sections. In addition, the researcher made presentations on cognitive vitality and aging at a local community center and a retirement community building, followed by an invitation to participate in the study. A total of 57 individuals expressed interest in the study over the phone, email, or in person.

Pre-screening. Interested individuals contacted the researcher by phone, email, or in person following the presentation. They were provided with a verbal or written description of the study and asked several questions related to eligibility criteria (age 65 
or older, English language fluency, having a study partner, etc.). The main reasons for not enrolling in the study were not being fluent in English, age under 65 years, not having a study partner, not being available for the duration of the study, absence of monetary compensation, and misunderstanding the information on the flyer.

Due to difficulty with recruitment, during the pre-screening stage the eligibility criteria were expanded to include an individual under the age of 65 (a 64-year old individual who was highly interested in participating but discontinued immediately after the pre-intervention assessment), and an individual on cognitive medication in the absence of neurological diagnosis. Also, several participants were highly interested in enrolling but did not have a study partner. They inquired whether they could be paired up with another older adult who did not have a study partner, and two pairs of participants were connected by the researcher. As a result, out of 57 individuals who inquired about the study, 22 (11 dyads) enrolled in the study and underwent screening and preintervention assessment.

The 22 participants were recruited from a variety of sources, as presented in Table 2. Flyers placed in public libraries and a senior center and in-person presentations on cognitive vitality led to the enrollment of most participants. Flyer placed in a coffee shop, an online advertisement, and referral by an existing participant resulted in enrollment of fewer participants. Following pre-screening over the phone, screening/testing sessions were scheduled with individuals who were interested and eligible to participate. 
Table 2.

Recruitment Sources. Recruitment Source Number of Dyads

Public libraries (flyers) 3

Senior center (flyers)

Retirement community (in person, following presentation)

Community center (in person, following a presentation)

1

Coffee shop (flyers)

Online community advertisement (CraigsList)

Snowball (referred by existing participants)

Total

Screening and pre-intervention assessment. Screening and pre-intervention assessment sessions were one-on-one and took 1-1.5 hours. These sessions took place at locations of participants' choice, including their home, library, or senior center. If the dyad were a married couple, one of the spouses waited in another room while the other one was being screened and tested. At the beginning of the screening and pre-test session, the participants were presented with the Informed Consent Form (Appendix 18) and provided study description verbally. The researcher went over each section of the consent form with the participants and addressed any questions or concerns. The participants were offered a copy of the consent form for their records. After signing the consent form, the researcher proceeded to asking screening/demographic questions (Appendix 19) and 
administering screening, cognitive, and self-report measures, as previously described in the Measures section.

Intervention. Session 1 of the intervention either followed the pre-intervention assessment immediately or was scheduled on a different day, depending on the participants' preferences. Session 1 was facilitated by the researcher and included both participants in the dyad. The session began with presenting the participants with materials including the manual, the sand clock, and writing utensils. The researcher demonstrated how to use the Log Sheet and encouraged the participants to schedule sessions on the regular basis and reschedule missed sessions. The participants were also asked to not have sessions two days in the row and allow at least one day between the sessions during any given week. The researcher showed the study partners how to administer tasks to one another, read the color-coded instructions, provide clues or correct answers, and how and when to take turns. The participants were encouraged to be patient and not competitive with one another, and to give each other positive feedback and encouragement. They were also asked to skip the task if it was too frustrating or was taking too long.

After Session 1 of the intervention, each dyad continued meeting on their own and the researcher kept in touch by phone or email. The cognitive training locations included home, senior center, conference room or common area of the building where the participants resided, and a coffee shop. When the dyads finished the first binder of the manual, the researcher met with participants and gave them the second binder of tasks. After the participants were finished with their Brain Training sessions, Post-Test Assessment and Interviews were scheduled. 
Post-intervention assessment. Post-intervention assessment was individual and included the same cognitive and self-report measures as the pre-intervention assessment. The screening measures of depression (PHQ-9) and cognitive status (MMSE) were readministered at post-test. Post-intervention testing sessions took about 40-50 minutes and were conducted individually at locations of participants' choice (home, library, coffee shop). If the dyad was a married couple, one of the participants waited in the other room while the other one was being tested.

Post-intervention interviews. Post-intervention interviews were also one-on-one and took place immediately after the post-test assessment. The interviews were semistructured (see Appendix 20) and took between 15 and 25 minutes. The questions focused on the participants' experience with the intervention and their opinions about its frequency and duration, the dyadic format, instructions and format of the manual, and the content of the training.

Incentives. No monetary compensation was provided to participants. At the end of the pre-intervention and post-intervention assessment sessions, the participants were offered to pick an item or two from a basket of small gifts, which included candles, coasters, picture frames, cosmetics, chap sticks, and other inexpensive items.

Data collection and processing. Each participant was assigned a code. The codes were used on all screening and assessment measures. The consent forms were stored separately from the measures and the codes were blacked out with a black marker so that the codes could not be connected with participant names in consent forms. The assessment instruments were administered in the paper-and-pencil format and scored manually. The scores and demographic information were entered into a password- 
protected Microsoft Excel spreadsheet, and then transferred into an IBM SPSS 21 (IBM Corporation, 2012) file without identifying information for coding and statistical analyses. The computer used for data entry and processing was password-protected.

All test scores and several demographic variables were entered as continuous variables. Categorical variables included gender, ethnicity, marital status, residential status, employment status, and dyad type. All variables were checked for normality and outliers, and parametric tests were supplied by nonparametric tests when the assumptions of normality or assumptions of parametric tests were violated. T-Tests have been demonstrated to be robust in extremely small samples and when assumptions are violated (De Winter, 2013). T-Tests were used to address Research Question 3 (pre-post changes in outcome measures) and supplied by Mann-Whitney test for independent groups or by Wilcoxon test for paired samples. Pearson's correlations were supplied or replaced by Spearman's correlation based if the distribution of a variables was not normal. $P$-value was set at .05 and confidence intervals were set at 95 percent, with effect sizes calculated where applicable. Bonferroni correction was applied for multiple comparisons.

Post-intervention interviews were audio-recorded using two digital recorders. The interviews were transcribed into Microsoft Word documents without identifying information and transferred into ATLAS.ti software (ATLAS.ti Scientific Software Development, 2014) for coding and theme analysis. The first interview was coded freely, which resulted in a number of codes. With each consecutive interview, the same codes were used or new codes were created and added as needed. After several interviews, it was possible to assign the codes to code families and derive a number of themes. After the coding process, codes, code families, and themes were revised to avoid redundancy. 


\section{RESULTS}

\section{Sample}

Participant screening. All participants performed above the cut-off score of 24 on a brief measure of cognitive status (MMSE). Several participants who read one or several words incorrectly as part of the $8^{\text {th }}$ grade-level English proficiency screening (SORT-3). Also, one participant scored in the severe range on a measure of depression (PHQ-9), but when asked, denied having depression or suicidal ideation and reported being monitored by a psychologist (no anti-depressant medication was prescribed). Lastly, several participants had cognitive measures administered within previous 1-3 years as part of medical research studies, but stated that none of the tests administered during pre-intervention assessment appeared to be the same as the ones they had previously taken. Despite these factors, none of the participants were excluded from the study due to difficulty recruiting and since external validity takes precedence over internal validity in feasibility research (Rounsaville \& Carroll, 2001).

Demographic characteristics. The sample $(N=18)$ was not well-balanced in terms of demographic characteristics, as majority were female $(N=12 ; 67 \%)$ and Caucasian $(N=15 ; 83 \%)$. Two of the participants were Latino and one was AfricanAmerican. The age ranged from 65 to 91 years $(M=75.94, S D=7.66)$, and the average education was above college-level $(M=16.94$ years, $S D=2.01$, range $=14-20)$. Nine of the participants were married, five were divorced or separated, three were widowed, and one was single. The majority of the participants resided either with their spouse $(N=9)$ or by themselves $(N=8)$, and one resided with a roommate. Twelve $(67 \%)$ older adults were retired, five $(28 \%)$ were still employed or self-employed, and one (6\%) was a 
homemaker. Fifteen $(83 \%)$ of the participants were native English speakers. As seen in Table 3, the majority of dyads were familiar adults, and one pair consisted of unfamiliar adults. All four spousal dyads consisted of a male and a female, while all non-spousal dyads consisted of participants of the same gender (one male pair and four female dyads).

Table 3.

Dyad Types.

\begin{tabular}{lcl}
\hline Dyad & Number of Dyads (dropped-out) & Gender \\
\hline Spouses & $4(1)$ & M \& F (all) \\
Friends & 2 & M \& M, F \& F \\
Neighbors & $2(1)$ & F \& F (all) \\
Unfamiliar & 1 & F \& F \\
Total & 9 & \\
\hline Note. $\mathrm{M}=$ male; F = female. & &
\end{tabular}

Participant retention. Simple percentage calculations were performed to address Hypothesis 1.1 (predicted 20 percent attrition rate). Out of 11 dyads that underwent preintervention assessment, two dyads discontinued immediately. The first dyad were unfamiliar older adults connected by the researcher. They spoke on the phone but never met in person. One of these participants was concerned about their age difference, difference in retirement status, and the fact that they lived far from one another. The second dyad were neighbors residing in the same building, and one of the participants withdrew due to health issues and a language barrier as her first language was Spanish, 
while her neighbor was a native English speaker. Both older adults in the first dyad and the native English speaker in the second dyad requested to be connected with another study partner, but the researcher was unable to find a different study partner for these individuals.

Out of nine dyads that began intervention, two dyads dropped out of the study after several sessions of Brain Training (attrition rate of 22 percent). One dyad was a married couple who had to discontinue due to one of the spouse's health problems. The other dyad were neighbors residing in the same retirement community and one of the participants passed away. The flow chart for study participants is presented in Figure 2.

Figure 2.

Study Flow Chart.

57 individuals interested

22 (11 dyads) underwent pre-intervention assessment

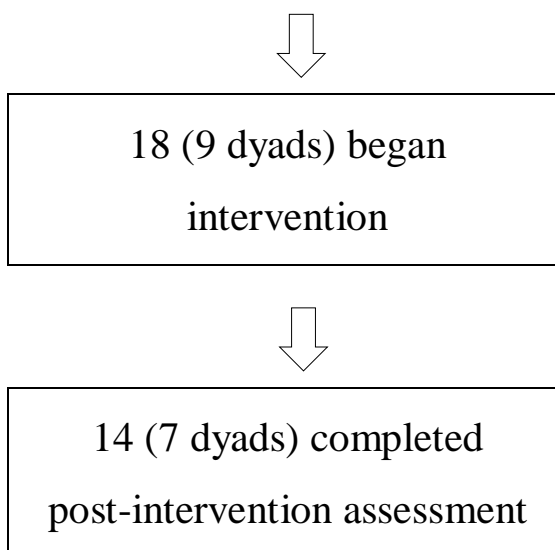


After excluding the four cases, sample $(N=14)$ characteristics remained relatively unchanged (age $M=74.36, S D=7.11$, range $=65-87$; education $M=17.00, S D=2.18$, range $=14-20$ ). Nine participants were female, five were male, 11 were Caucasian, two were Latino, and one was African-American. Seven individuals were married and seven were single, widowed, divorced, or separated, and four older adults were still employed.

Participant adherence. Again, simple percentage calculations were performed to evaluate Hypothesis 1.2 (adherence rate of 80 percent). During the course of the intervention, two of the dyads requested to finish the intervention early due to high participant burden (frequency and duration) and some redundancy of the tasks. One of these dyads were neighbors and the other one were unfamiliar participants who completed 12 and 9 sessions, respectively. It became apparent that there were not enough tasks for 24 sessions, as one dyad (married couple) moved very fast through the tasks and completed all of them in 13 sessions, while another dyad (friends) completed all tasks in 21 sessions. Another dyad (friends) were running out of tasks and despite the researcher's recommendations to complete as many sessions as it took to finish the tasks, they decided to use fewer tasks per session to ensure that they completed all 24 sessions. As a result, their sessions were less than an hour long towards the end of the intervention. The resulting number of sessions for the sample ranged from 9 to $24(M=15.14, S D=5.30)$, and the duration of intervention ranged from 4 to 21 weeks $(M=12.21, S D=5.44)$.

It is worth noting that although the eligibility criteria included absence of neurological or cognition-related cognition, due to difficulty recruiting one participant was enrolled despite being on cognitive medication (daily patch Exelon) over the previous 7 years. The medication was prescribed due to the participant's self-report of 
mild decline in the absence of a MCI or dementia diagnosis. Another participant was diagnosed with a brain tumor and operated on shortly after completing the study.

\section{Statistical Analyses}

Statistical analyses were performed using IBM SPSS, $21^{\text {st }}$ edition (IBM Corporation, 2012). Confidence intervals were set at 95\%, with significance value $p=$ .05. Bonferroni correction was employed for multiple comparisons (Field, 2013). Effect sizes were represented by $r$ values for Pearson's and Spearman's correlation, MannWhitney Test, and Wilcoxon Test, with conventional values of .1, .3, and .5 representing small, medium, and large effect size. For T-Tests, Cohen's $d$ was used, with conventional values of $.2, .5$, and .8 representing small, medium, and large effect size (Cohen, 1969).

Partial eta squared, defined as the proportion of variance explained by a variable when excluding other variables (Field, 2013), was used for $F$-tests. Because partial eta squared values are typically inflated compared to eta squared values (Morris \& Fritz, 2013), conventions for eta squared $(.01, .06$, and 1.14 representing small, medium, and large effect size, Cohen, 1973) are considered inappropriate for partial eta squared. For this reason, partial eta squared values were evaluated based on Morris and Fritz' (2013) recommendations, who analyzed data from 224 memory studies with 2171 partial eta squared statistics and suggested the values of $.08, .18$, and .41 for small, medium, and large effect sizes of partial eta squared.

\section{Data Screening}

All data were screened for normality and outliers. Shapiro-Wilk test of normality demonstrated that years of education variable was not normally distributed. Among cognitive measures, normality assumption was violated for pre-test MMSE scores, pre- 
test RBANS-U Delayed Memory domain, post-test Visuospatial/Constructional domain, post-test Language domain, post-test List Learning subtest, and post-test Story Memory subtest scores. This was also the case when the four participants who dropped out were excluded from the pre-test sample. Visual examination of histograms confirmed these findings. Boxplot graphs revealed mild outliers on pre-test RBANS-U Language domain, pre-test Delayed Memory domain, and post-test TMT B. As for the self-report measures, nearly all scores violated the assumption of normality, as assessed by the Shapiro-Wilk test. Also, the majority of self-report variables were marked by mild and/or extreme outliers.

Although outliers affect the mean value and inflate the standard error increasing the risk of type I error in parametric tests (Parker, 2006), excluding these cases from the analyses or modifying problematic values would be inappropriate because they are true representations of participant scores and because of the small sample size. For this reason, group median scores are included in summary tables for self-report measures in addition to means, as they may be more representative of the sample. Also, parametric tests are supplied by non-parametric tests when the analyses involve cognitive measures that were non-normal or had outliers, and non-parametric tests are utilized for the analyses of self-report measures.

Retention and adherence variables. Analyses were performed to assess whether the four participants who dropped out during the course of the intervention differed from the rest of the sample. According to De Winter (2013), who ran numerous simulated analyses on small samples, One-Sample or Two-Sample T-Test has adequate power and error rate even in cases where samples or groups are extremely small $(N \leq 5)$, group sizes 
are unequal, and/or unequal variances assumption is violated. In the light of these findings, Independent Samples T-Tests were performed to compare the four participants who dropped out to the rest of the sample.

The results of the T-Tests demonstrated that those who dropped out did not significantly differ from the other participants in terms of their age, education, MMSE, Stroop, and TMT scores. However, the participants who dropped out scored lower on the RBANS-U Attention domain (dropped out $M=95.50, S D=3.87$, retained $M=108.79$, $S D=15.13 ; T(16)=-2.96, S E=4.48, p=.009,95 \% C I[-22.79,-3.78])$ and the Digit Span subtest that factors into the Attention domain (dropped out $M=7.75, S D=1.50$, retained $M=11.29, S D=2.49 ; T(16)=-2.96, S E=1.33, p=.017,95 \% C I[-6.35,-.72])$. The effect sizes for both variables were large, with Cohen's $d=1.20$ for the Attention domain and 1.72 for the Digit Span subtest.

Additionally, it was found that individuals who dropped-out had significantly lower scores on RAND SF-36 Physical Functioning (dropped-out $M=30.00, S D=17.80$, retained $M=75.93, S D=26.28 ; T(16)=-3.25, S E=14.12,95 \% C I[-75.87,-15.99], p=$ .005 , Cohen's $d=2.05)$. Mann-Whitney test for independent samples confirmed group differences for Rand SF-36 Physical Functioning $(U=4.00, Z=-2.56, p=.008)$. Effect size $r$ for Mann Whitney test was calculated by dividing the $Z$ value by the square root of the sample $N$ (Field, 2013), which resulted in a large effect size $r=-.60$.

With regard to adherence, Pearson's correlations were performed to identify any relationships between number of sessions completed or the number of weeks of intervention and Stroop, TMT, and RBANS- U Total and domain scores $(N=14)$. The findings indicated that the number of sessions completed was significantly, negatively 
related to Pre-Test Stroop Color $(R=-.61, p=.021)$ and Post-Test Stroop Color scores $(R$ $=-.55, p=.041)$, pre-test RBANS-U Immediate Memory domain $(R=-.73, p=.003)$, pre-test RBANS-U Attention domain $(R=-.57, p=.034)$, post-test RBANS-U Total score $(R=-.66, p=.011)$, post-test RBANS-U Immediate Memory domain $(R=-.66, p=$ $.010)$, and post-test RBANS-U Delayed Memory domain $(R=-.53, p=.049)$. When correlations were performed for number of weeks of intervention, a slightly different pattern was observed. Weeks of intervention were significantly, negatively associated with pre-test Stroop Word $(R=-.66, p=.010)$, post-test Stroop Word $(R=-.66, p=$ $.011)$, post-test Stroop Color scores $(R=-.64, p=.014)$, pre-test RBANS-U Immediate Memory domain $(R=-.75, p=.002)$, and post-test RBANS-U Language domain $(R=-$ $.74, p=.002)$.

To assess associations between numbers of sessions/ number of weeks of intervention and self-report measures, Spearman's rank correlations were employed. It was found that larger number of sessions correlated with lower level of emotional functioning at pre-test (RAND SF-36 Emotional $R=-.84, p=.037)$ and post-test $(R=-$ $.89, p=.018$ ), and higher level of pain at post-test (RAND SF-36 Pain $R=-.89, p=$ .018). Longer duration of intervention was associated with lower post-test social participation (WHOQOL-OLD Social Participation $R=-.91, p=.013$ ), higher concern about dying at pre-test (WHOQOL-OLD Death and Dying $R=-.84, p=.038$ ) and posttest $(R=-.87, p=.026)$, lower post-test overall health (RAND SF-36 General Health $R=$ $-.84, p=.038)$, and lower pre-test daily functioning as reported by the spouse (B-ADL $R$ $=-.97, p=.001)$. 


\section{Pre-Intervention Assessment}

Cognitive measures. The analyses of pre-test scores were performed for exploratory purposes with $N=18$ (all participants who began the intervention). Table 4 presents descriptive statistics for the sample pre-test scores on cognitive measures, including minimum and maximum scores, means, standard deviations, and lower and upper limits of the $95 \%$ confidence interval. It should be noted that four of the RBANS-U subtests are scored as a percentile range (e.g., 25-50\%, 0-2\%, etc., as per publisher): Line Orientation, Picture Naming, List Recall, and List Recognition. For illustrative purposes, these four subtests were included in Table 4 and coded based on percentiles as follows: below $25 \%=1,25-50 \%=2,51-75 \%=3$, above $75 \%=4$. However, these subtests were excluded from parametric and non-parametric statistical analyses.

As a group, the participants' mean MMSE score was normal $(M=28, S D=1.33)$, nearing the maximum score of 30. Stroop and TMT measures were scored using the Mayo's Older Adults Normative Studies (MOANS) norming data (Ivnik et al., 1996), the most comprehensive and detailed norming report in older population with 3-year intervals for age groups (i.e., 66-68 years, 69-71 years, etc.) based on a nationally representative sample of 746 older adults. Since it is known that the population means for Stroop and TMT equal 10, One-Sample T-Tests were used to compare the group mean scores to the population mean. The results showed that relative to the population mean, the sample scored higher on the TMT B $(M=11.33, S D=2.35 ; T(17)=2.41, p=.028$, $95 \%$ CI $[.16,2.50])$.

In terms of the comprehensive neuropsychological battery RBANS-U, the sample Total score $(M=96.78, S D=13.18)$ was close to the population mean of 100 and 
standard deviation of 15 . Figure 3, which presents a histogram of the participants' Total RBANS-U scores, demonstrates that the distribution of scores was roughly normal. Domain mean scores were also generally close to the population mean of 100 and standard deviation of 15, with the exception of Visuospatial/Constructional domain score which fell nearly 10 points below the population mean and was more scattered $(M=$ 91.17, $S D=18.05)$. One-Sample T-Tests comparing RBANS-U Total and Domain sample means to the population mean of 100 revealed that the mean difference approached significance for the Visuospatial Domain $(M=91.17, S D=18.05 ; T(17)=-$ $2.08, p=.053,95 \% C I[-17.81, .14])$. One-Sample T-Tests comparing sample subtest means to the population mean of 10 demonstrated that the participants scored significantly lower on the Figure Copy subtest $(M=8.00, S D=3.22 ; T(17)=-2.64, p=$ $.017,95 \% C I[-3.60,-.40])$ and Figure Recall subtest $(M=8.44, S D=2.36 ; T(17)=-$ $2.80, p=.012,95 \% C I[-2.73,-.38])$. 
Table 4.

Descriptive Statistics for Cognitive Test Scores at Pre-Intervention Assessment.

\begin{tabular}{|c|c|c|c|c|c|c|}
\hline \multirow[t]{2}{*}{ Measure } & \multirow[t]{2}{*}{ Min. } & \multirow[t]{2}{*}{ Max. } & \multirow[t]{2}{*}{$M$} & \multirow[t]{2}{*}{$S D$} & \multicolumn{2}{|c|}{$95 \% C I$} \\
\hline & & & & & Lower & Upper \\
\hline MMSE (30 points maximum) & 25 & 30 & 28.00 & 1.33 & 27.34 & 28.66 \\
\hline \multicolumn{7}{|l|}{ Stroop Color-Word Test ${ }^{\mathrm{a}}$} \\
\hline Word (word reading) ${ }^{\mathrm{b}}$ & 6 & 14 & 10.18 & 2.48 & 8.90 & 11.45 \\
\hline Color (color naming) & 2 & 13 & 8.56 & 3.00 & 7.07 & 10.04 \\
\hline Color-Word (word inhibition) & 5 & 16 & 10.50 & 3.35 & 8.84 & 12.16 \\
\hline \multicolumn{7}{|l|}{ Trail Making Test ${ }^{\mathrm{a}}$} \\
\hline Part A (numbers only) & 6 & 16 & 10.67 & 2.30 & 9.52 & 11.81 \\
\hline Part B (numbers and letters) & 6 & 16 & 11.33 & 2.35 & 10.16 & 12.50 \\
\hline \multicolumn{7}{|l|}{ RBANS-U } \\
\hline Total Score ${ }^{c}$ & 68 & 126 & 96.78 & 13.18 & 90.22 & 103.33 \\
\hline \multicolumn{7}{|l|}{ RBANS-U Domains ${ }^{c}$ : } \\
\hline Immediate Memory & 65 & 129 & 94.56 & 15.74 & 86.73 & 102.38 \\
\hline Visuospatial/Constructional & 66 & 126 & 91.17 & 18.05 & 82.19 & 100.14 \\
\hline Language & 87 & 125 & 100.78 & 9.82 & 95.89 & 105.66 \\
\hline Attention & 82 & 132 & 105.83 & 14.49 & 98.63 & 113.04 \\
\hline Delayed Memory & 71 & 117 & 97.28 & 11.06 & 91.78 & 102.78 \\
\hline \multicolumn{7}{|l|}{ RBANS-U Subtests ${ }^{\mathrm{d}}$ : } \\
\hline List Learning & 3 & 15 & 9.33 & 3.33 & 7.68 & 10.99 \\
\hline Story Memory & 4 & 15 & 8.83 & 2.98 & 7.35 & 10.31 \\
\hline Figure Copy & 2 & 14 & 8.00 & 3.22 & 6.40 & 9.60 \\
\hline Line Orientation $^{\mathrm{e}}$ & 1 & 4 & 2.39 & 1.29 & 1.75 & 3.03 \\
\hline Picture Naming ${ }^{\mathrm{e}}$ & 1 & 4 & 2.83 & .99 & 2.34 & 3.32 \\
\hline Semantic Fluency & 5 & 16 & 10.00 & 2.87 & 8.57 & 11.43 \\
\hline Digit Span & 6 & 15 & 10.50 & 2.73 & 9.14 & 11.86 \\
\hline Coding & 7 & 16 & 11.33 & 2.87 & 9.91 & 12.76 \\
\hline List Recall $^{\mathrm{e}}$ & 1 & 4 & 2.44 & 1.15 & 1.87 & 3.02 \\
\hline List Recognition ${ }^{\mathrm{e}}$ & 1 & 3 & 2.17 & .71 & 1.82 & 2.52 \\
\hline Story Recall & 5 & 12 & 9.11 & 2.40 & 7.92 & 10.30 \\
\hline Figure Recall & 5 & 14 & 8.44 & 2.36 & 7.27 & 9.62 \\
\hline
\end{tabular}

Note $. N=18$. Max. $=$ maximum score; Min. $=$ minimum score MMSE $=$ Mini Mental State Examination; RBANS-U $=$ Repeatable Battery for the Assessment of Neuropsychological Status-Updated.

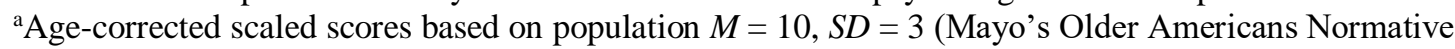
Studies, Ivnik et al., 1996).

${ }^{\mathrm{b}} N=17$ (1 missing).

${ }^{c}$ Age-corrected index scores based on population $M=100, S D=15$ (publisher norms). 
${ }^{\mathrm{d}}$ Age-corrected scaled scores based on population $M=10, S D=3$ (publisher norms).

'Percentage range rather than scaled scores are provided by publisher; these were coded as $1=$ below $25 \%$, $2=25-50 \%, 3=51-75 \%, 4=$ above $75 \%$ for illustrative purposed

Figure 3.

Frequency Distribution for the Total Score on the Repeatable Battery for the Assessment of Neuropsychological Status-Updated (RBANS-U).

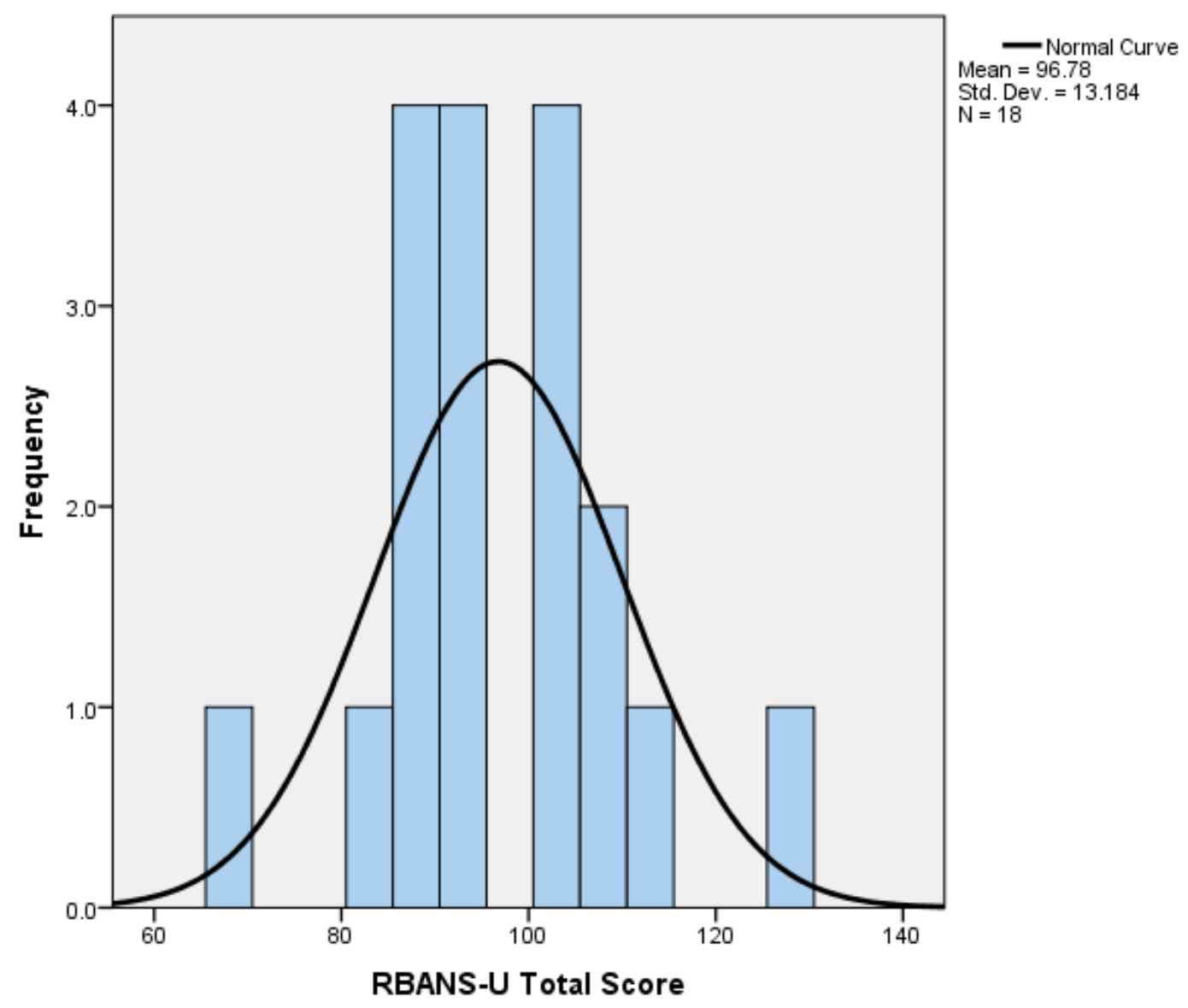

Paired-samples T-Tests on pre-test RBANS-U Total and domain scores were used to determine whether as a sample, the participants varied in how they function in different areas of cognition. Relative to their Total RBANS-U score $(M=96.78, S D=$ 13.18), the sample performed significantly higher on RBANS-U Attention $(M=105.78$, 
$S D=14.49 ; T(17)=-4.21, p=.001, S E=2.15,95 \% C I[-13.60,-4.52])$. Also, their Attention mean score was significantly higher than their Immediate Memory $(M=94.56$, $S D=15.74 ; T(17)=-3.12, S E=3.63, p=.006,95 \% C I[-18.93,-3.62])$, Visuospatial $(M$ $=91.17, S D=18.05 ; T(17)=-3.32, S E=4.42, p=.004,95 \% C I[-24.00,-5.34])$, and Delayed Memory $(M=97.28, S D=11.06 ; T(17)=2.60, S E=3.30, p=.019,95 \% C I$ $[-1.60,15.51])$ scores. Also, the participants scored significantly lower on the Visuospatial domain compared to the Language domain $(M=100.78, S D=9.82 ; T(17)=$ $-2.25, S E=4.27, p=.038,95 \% C I[-18.61,-.61])$. Figure 4 displays sample means for the five RBANS-U domains, as well as lines representing the population mean and the sample mean.

Because two of the RBANS-U domain scores at pre-test were marked by nonnormality and/or mild outliers (i.e., pre-test RBANS-U Language and Delayed Memory), the analyses were repeated using Wilcoxon's Related Samples. The results confirmed that the participants' attention skills were stronger than their delayed memory $(Z=-2.77, p=$ $.023, r=.65)$, and that their language skills were stronger than their visuospatial reasoning $(Z=-1.98, p=.047, r=.47)$. The $r$ values of Wilcoxon tests represent medium and large effect sizes, calculated by dividing the $Z$ value by the square root of the sample $N$ (Field, 2013). 
Figure 4.

Sample Means for the Five Domains of the Repeatable Battery for the Assessment of Neuropsychological Status-Update (RBANS-U).

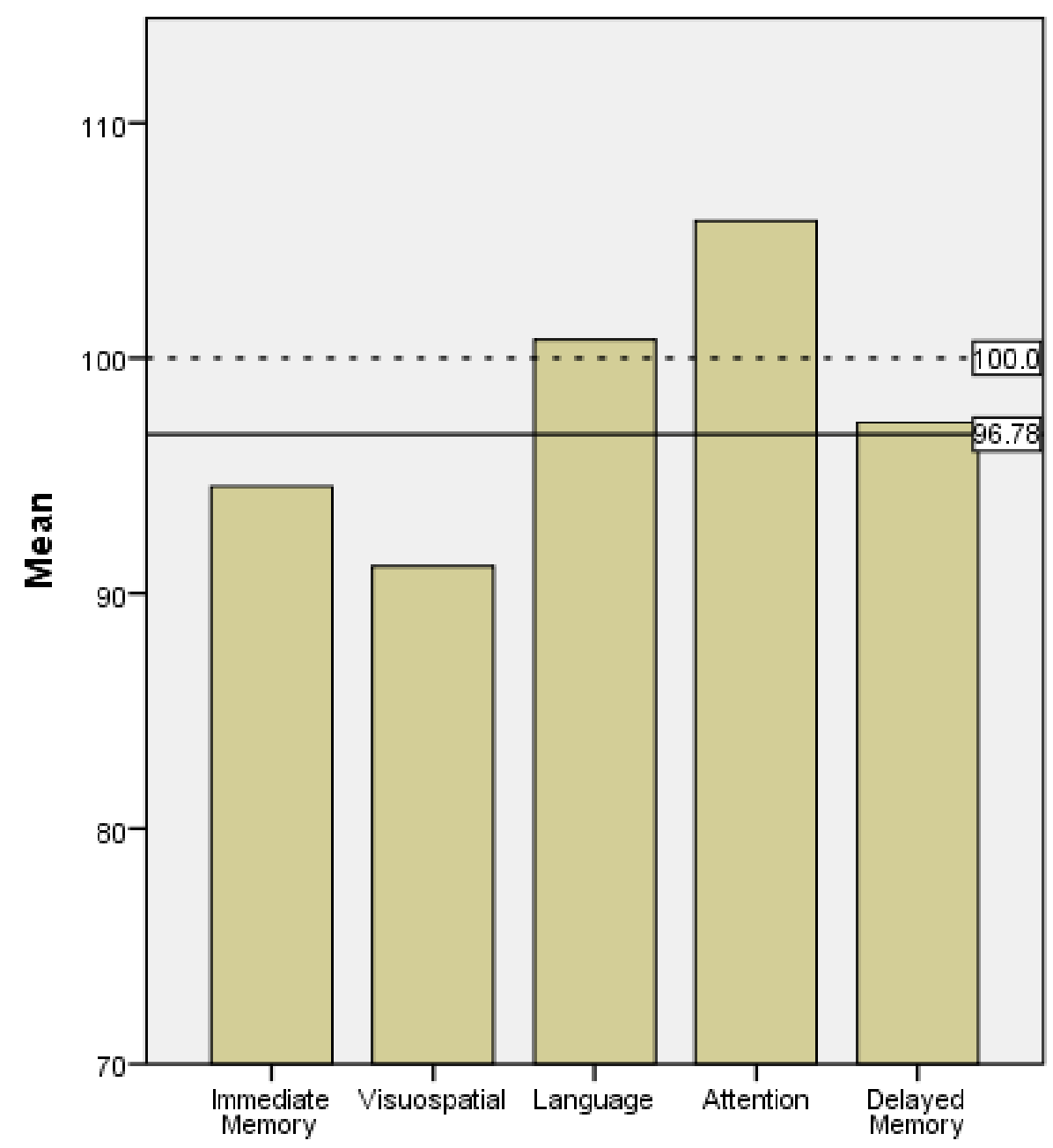

Note. The solid line represents the sample mean of 96.78 , and the dotted line represents the population mean of 100 .

Also for exploratory purposes, bivariate Pearson's correlations were employed to evaluate relationships among pre-intervention cognitive test scores, including MMSE, Stroop, TMT, and RBANS-U Total and Domain scores. As expected, there were 
significant positive large correlations between different parts of the same tests, including three parts of the Stroop and TMT A and TMT B scores (see Table 5). Positive correlations were also found between RBANS-U total score and each of the RBANS-U domain scores. The different RBANS-U domain scores were not significantly related, confirming that they assess distinct cognitive functions, with the exception of the correlation between RBANS-U Immediate Memory and Delayed Memory scores $(R=$ $.58, p=0.011)$.

As seen in Table 5, the sample Stroop Word score was positively correlated with RBANS-U Immediate Memory domain score, while the Stroop Color score was positively correlated with RBANS-U Language, Attention, and Total scores. TMT B score was related to RBANS-U Immediate Memory, Attention, Delayed Memory, and Total scores. These correlations exemplify the overlapping roles of executive functions, including attention, working memory, and processing speed, as well as their connection with other functions such as delayed memory and language.

MMSE scores were not significantly related to any of the cognitive measures. One correlation that approached significance was between MMSE and TMT A $(R=.44$, $p=0.066)$. Due to the non-normal distribution of MMSE scores, Spearman's Rho correlations were performed between MMSE and other cognitive scores. The results showed that there was a significant positive correlation between MMSE and TMT A only $(R=.52, p=.027)$. The fact that only one cognitive score was associated with performance on MMSE confirms that MMSE is a general tool that is not sensitive enough to detect subtle cognitive decline, is insufficient for the assessment of neuropsychological status, and should be utilized for screening purposes only. 
Table 5.

Bivariate Correlations among Cognitive Measures.

\begin{tabular}{|c|c|c|c|c|c|c|c|c|c|c|c|c|c|}
\hline & \multicolumn{2}{|c|}{ MMSE } & \multicolumn{4}{|c|}{$\underline{\text { Stroop }}$} & \multicolumn{2}{|c|}{$\underline{\text { TMT }}$} & \multicolumn{5}{|c|}{$\underline{\text { RBANS-U }}$} \\
\hline & & & $\mathrm{W}$ & $\mathrm{C}$ & $\mathrm{CW}$ & Int & A & $\mathrm{B}$ & IM & VS & LA & $\mathrm{AT}$ & $\mathrm{DM}$ \\
\hline \multicolumn{14}{|c|}{ Stroop } \\
\hline & $R$ & $.25^{\mathrm{a}}$ & & & & & & & & & & & \\
\hline & $p$ & .331 & & & & & & & & & & & \\
\hline \multirow[t]{2}{*}{$\mathrm{C}$} & $R$ & .22 & $.51^{\mathrm{a} *}$ & & & & & & & & & & \\
\hline & $p$ & .376 & .038 & & & & & & & & & & \\
\hline \multirow[t]{2}{*}{$\mathrm{CW}$} & $R$ & .08 & $.41^{\mathrm{a}}$ & $.75^{* *}$ & & & & & & & & & \\
\hline & $p$ & .754 & .103 & $<.001$ & & & & & & & & & \\
\hline \multicolumn{14}{|l|}{$\underline{\mathrm{TMT}}$} \\
\hline \multirow[t]{2}{*}{$\bar{A}$} & $R$ & .44 & $.27^{\mathrm{a}}$ & .40 & .26 & .33 & & & & & & & \\
\hline & $p$ & .066 & .301 & .096 & .298 & .181 & & & & & & & \\
\hline \multirow[t]{2}{*}{ B } & $R$ & .21 & $.15^{\mathrm{a}}$ & .36 & .46 & .20 & $.53^{*}$ & & & & & & \\
\hline & $p$ & .410 & .563 & .147 & .057 & .439 & .023 & & & & & & \\
\hline \multicolumn{14}{|c|}{$\underline{\text { RBANS-U }}$} \\
\hline \multirow[t]{2}{*}{ Total } & $R$ & .22 & $.35^{\mathrm{a}}$ & $.57^{*}$ & .41 & .15 & .36 & $.70^{* * *}$ & & & & & \\
\hline & $p$ & .377 & .166 & .013 & .091 & .541 & .147 & .001 & & & & & \\
\hline \multirow[t]{2}{*}{ IM } & $R$ & .07 & $.50^{\mathrm{a} *}$ & .37 & .29 & .09 & .40 & $.63^{* *}$ & $.70^{* * *}$ & & & & \\
\hline & $p$ & .799 & .039 & .127 & .239 & .722 & .097 & .005 & .001 & & & & \\
\hline \multirow[t]{2}{*}{ VS } & $R$ & .13 & $-.19^{a}$ & .33 & .21 & .10 & .16 & .45 & .11 & $.66^{* *}$ & & & \\
\hline & $p$ & .607 & .471 & .187 & .406 & .696 & .530 & .062 & .657 & .003 & & & \\
\hline \multirow[t]{2}{*}{ LA } & $R$ & -.04 & $.10^{\mathrm{a}}$ & $.47^{*}$ & .34 & -.06 & .14 & .40 & .36 & .27 & $.61^{* *}$ & & \\
\hline & $p$ & .887 & .713 & .049 & .167 & .826 & .595 & .097 & .145 & .283 & .008 & & \\
\hline \multirow[t]{2}{*}{$\mathrm{AT}$} & $R$ & .37 & $.40^{\mathrm{a}}$ & $.52^{*}$ & .41 & .26 & .46 & $.53^{*}$ & $.48^{*}$ & .35 & $.49^{*}$ & $.79^{* *}$ & \\
\hline & $p$ & .134 & .114 & .027 & .089 & .290 & .053 & .023 & .042 & .152 & .042 & $<.001$ & \\
\hline \multirow[t]{2}{*}{$\mathrm{DM}$} & $R$ & .22 & $.46^{\mathrm{a}}$ & .31 & .11 & -.01 & .18 & $.52^{*}$ & $.58^{*}$ & .40 & .24 & .43 & $.74^{* *}$ \\
\hline & $p$ & .389 & .062 & .206 & .658 & .970 & .470 & .027 & .011 & .099 & .342 & .078 & $<.001$ \\
\hline
\end{tabular}

Note. $N=18$. MMSE $=$ MiniMental State Examination; $\mathrm{W}=$ Stroop Word; $\mathrm{C}=$ Stroop Color $\mathrm{CW}=$ Stroop Color-Word; TMT = Trail Making Test; RBANS-U = Repeatable Battery for the Assessment of

Neuropsychological Status-Updated; IM = Immediate Memory; VS = Visuospatial Processing; LA = Language; $\mathrm{AT}=$ Attention; $\mathrm{DM}=$ Delayed Memory .

${ }^{\mathrm{a}} \mathrm{N}=17$

$* \mathrm{p}<0.05$

$* * \mathrm{p}<0.01$ 
Demographic variables. Analyses were performed to address Hypothesis 4.1 (lower education, older age, and lower health status associated with lower pre-test cognitive scores; no link with gender). Bivariate correlations demonstrated that the participants' age was significantly positively correlated with their RBANS-U Language domain scores only $(R=.56, p=0.016)$. Interestingly, educational level was not related to any of the cognitive measures. Because education variable was not normally distributed, Spearman's correlations were also performed and confirmed lack of association between education and cognition. Independent-Samples T-Tests were employed to determine whether males and females differed on any of the cognitive measures. Unless noted otherwise, the assumption of equal variances was not violated. Independent-Samples T-Test did not detect gender differences in age and education (females $N=12$, males $N=6$ ). Because education variable violated the assumption of normality, same factors were analyzed using Mann-Whitney test. Again, no gender differences on age or education were found. Additionally, there were no gender differences on MMSE, Stroop, or TMT. On the RBANS-U, males scored higher than females on the Visuospatial/Constructional domain (males $M=104.00, S D=21.01$; females $M=84.75, S D=12.98 ; T(16)=-2.42, S E=7.96, p=.028,95 \% C I[-36.13,-$ 2.37], Cohen's $d=1.21$ ), Figure Copy subtest, which factors into the Visuospatial domain (males $M=10.17, S D=3.25$; females $M=6.92, S D=2.71 ; T(16)=-2.25, S E=$ $1.45, p=.039,95 \% C I[-6.32,-.19]$, Cohen's $d=1.13)$, and Figure Recall subtest, which factors into the Delayed Memory domain (males $M=10.33$, $S D=2.73$; females $M=$ 7.50, $S D=1.51 ; T(16)=-2.87, S E=.99, p=.011,95 \% C I[-4.93,-.74]$, Cohen's $d=$ 1.43). As indicated by the Cohen's $d$ values, the effect sizes were large. 
Married individuals living with a spouse $(N=9)$ were also compared to those who were not married/ not living with a partner (single, widowed, divorces, or separated; $N=$ 9). Independent-samples T-Test demonstrated that while no differences were found on age, married older adults tended to have more years of education (married $M=18.22, S D$ $=1.86$; not married $M=15.67, S D=1.23 ; T(16)=3.45, S E=.74, p=.003,95 \% C I[.98$, 4.13], Cohen's $d=1.62$ ). Because education variable violated the assumption of normality, the same analyses were repeated using Mann-Whitney test, which confirmed significant group differences on education $(U=11.50, Z=-2.70, p=.008, r=.64)$. With regard to the cognitive test scores, T-Tests revealed significant group differences only on RBANS-U Visuospatial domain (married $M=96.67, S D=17.48$; not married $M=85.67$, $S D=17.86 ; T(16)=1.32, S E=1.36, p=.036,95 \% C I[.236,5.99]$, Cohen's $d=1.43)$.

Similarly, T-Tests were performed to identify differences among retired and employed participants. No group differences were found on age and education, although the age differences approached significance (retired $M=78.50, S D=7.43$, employed $M=$ 70.60, $S D=6.19, T(15)=2.09, S E=3.79, p=.055)$. Because education variable violated the assumption of normality, the same analyses were repeated using Mann-Whitney test, which showed that while there were no significant group differences on education $(U=$ 24.50, $Z=-.61, p=.57, r=.14)$, married participants tended to be older $(U=11.00, Z=-$ $2.01, p=.048, r=.47)$. Group differences were found on RBANS-U Language domain score and Semantic Fluency subtest, as indicated by T-Tests. Specifically, retired participants performed higher than those who were still employed on RBANS-U Language domain (retired $M=104.33, S D=9.98$; employed $M=93.20, S D=4.55 ; T(15)$ $=2.36, S E=4.72, p=.032,95 \% C I[1.08,21.16]$, Cohen's $d=1.26)$ and on RBANS-U 
Semantic Fluency subtest, which is included in the calculation of the Language domain score (retired $M=11.00, S D=2.41 ;$ employed $M=7.00, S D=1.58 ; T(15)=3.38, S E=$ $1.18,95 \% C I[1.48,6.52], p=.004$, Cohen's $d=1.80$ ), with large effect sizes. Because Language scores had an outlier, Mann-Whitney test was utilized and confirmed the results on this measure $(U=8.50, Z=-2.28, p=.019, r=.54)$. These results align with the previous finding of significant positive relationship between age and RBANS-U Language, since retired participants were older.

To address another component of Hypothesis 4.1 (link between cognition and health status), Spearman's bivariate correlations were employed to determine whether MMSE, Stroop, TMT, and RBANS-U Total and Domain scores correlated with any of the self-report scores. It was found that lower performance on Stroop Color-Word was associated with higher levels of pain (RAND SF-36 $r=.49, p=.040$ ). Also, lower RBANS-U Language domain score was related to lower overall quality of life (WHOQOL-OLD Total $r=-.49, p=.048$ ), lower sensory functioning (WHOQOL Sensory $r=.62 p=.006$ ), higher concern about dying (WHOQOL-OLD Death and Dying $r=-.53, p=.022$ ), lower overall health (RAND SF-36 General Health $r=.59, p=$ .011 ), and lower energy (RAND SF-36 Energy/Fatigue $r=-.48, p=.045$ ). Lastly, lower RBANS-U Visuospatial domain scores were also related to lower WHOQOL Sensory Functioning scores $(r=-.48, p=.045)$. However, none of the correlations were significant when the Bonferroni correction was employed.

Self-report measures. Table 6 presents descriptive statistics for pre-test selfreport scores. Medians are included as they may be better estimates of central tendency due to non-normality and outliers in the self-report data. Median values show that as a 
whole, sample had low level of depression and relatively high levels of quality of life, as the medians for WHOQOL-OLD Total and domain scores were 75 or above (maximum score indicating highest levels of quality of life $=100$ ). Relative to their total quality of life score (WHOQOL-OLD Total), the sample reported higher levels of Sensory Functioning (Wilcoxon $Z=-2.20, p=.028, r=.53$ ) and higher levels of Autonomy (Wilcoxon $Z=-2.52, p=.012, r=.61$ ).

Similarly, the maximum score for RAND SF-36 is 100, which represents highest level of perceived health. Median values for different aspects of bio-psycho-social functioning, as measured by RAND SF-36 were also mostly 75 or above, with the exception of the median score of 66 on the Energy/Fatigue domain and 74 on the Pain domain. Because RAND SF-36 does not provide a total score, analysis of relatively low and high scores was not possible for this measure. The lowest possible score on a measure of daily functioning (B-ADL) is 1 which represents absence of difficulties in ADLs and IADLs. The sample median B-ADL scores were also close to 1, indicating low level of difficulty in daily functioning. Because the self-report data were not normal, Spearman's correlations were used to explore relationships among the self-report measures. As expected, the vast majority of self-report measures were significantly, positively correlated. 
Table 6

Descriptive Statistics for Self-Report Measures.

\begin{tabular}{|c|c|c|c|c|c|c|c|c|}
\hline & & & & & & & \multicolumn{2}{|c|}{$\underline{95 \% C I}$} \\
\hline & Min. & Max. & Med. & $M$ & $S D$ & $S E$ & Lower & Upper \\
\hline PHQ9 ${ }^{\mathrm{a}}$ (depression) & 0 & 26 & 2.00 & 3.67 & 6.27 & 1.48 & 0.55 & 6.78 \\
\hline \multicolumn{9}{|l|}{$\mathrm{B}-\mathrm{ADL}^{\mathrm{b}}$ (daily functioning) } \\
\hline Self-report & 1.00 & 8.72 & 1.40 & 1.89 & 1.77 & 0.42 & 1.01 & 2.78 \\
\hline Informant report $^{\mathrm{c}}$ & 1.00 & 3.36 & 1.34 & 1.63 & 0.80 & 0.28 & 0.96 & 2.30 \\
\hline \multicolumn{9}{|l|}{ WHOQOL-OLD ${ }^{\mathrm{d}}$ (quality of life) } \\
\hline Total Score & 9 & 95 & 81.00 & 75.94 & 20.03 & 4.86 & 65.64 & 86.24 \\
\hline Sensory Functioning & 0 & 100 & 91.00 & 81.39 & 25.28 & 5.96 & 68.82 & 93.96 \\
\hline Autonomy & 6 & 100 & 81.00 & 78.96 & 20.63 & 4.86 & 68.70 & 89.22 \\
\hline Abilities $^{\mathrm{e}}$ & 0 & 100 & 81.00 & 75.47 & 25.36 & 6.15 & 62.43 & 88.51 \\
\hline Social Participation $^{\mathrm{e}}$ & 25 & 100 & 75.00 & 71.47 & 20.92 & 5.07 & 60.72 & 82.23 \\
\hline Death and Dying & 25 & 100 & 78.00 & 72.72 & 23.43 & 5.52 & 61.07 & 84.37 \\
\hline Intimacy $^{\mathrm{e}}$ & 0 & 100 & 75.00 & 71.41 & 24.34 & 5.90 & 58.90 & 83.93 \\
\hline \multicolumn{9}{|l|}{ RAND SF-36 ${ }^{\mathrm{d}}$ (health status) } \\
\hline Physical & 5 & 100 & 75.00 & 65.72 & 31.14 & 7.34 & 50.23 & 81.21 \\
\hline Role Limit. due to Physical Symptoms & 0 & 100 & 75.00 & 65.28 & 36.52 & 8.61 & 47.12 & 83.44 \\
\hline Role Limit. due to Emotional Symptoms & 0 & 100 & 100.00 & 79.72 & 32.58 & 7.68 & 63.52 & 95.92 \\
\hline Energy/Fatigue & 0 & 90 & 66.00 & 61.78 & 23.54 & 5.55 & 50.07 & 73.48 \\
\hline Emotional & 4 & 96 & 86.00 & 81.11 & 20.57 & 4.85 & 70.88 & 91.34 \\
\hline Social & 0 & 100 & 100.00 & 82.72 & 30.90 & 7.28 & 67.36 & 98.09 \\
\hline Pain & 10 & 90 & 74.00 & 67.22 & 25.09 & 5.91 & 54.75 & 79.70 \\
\hline General Health & 25 & 95 & 77.50 & 72.00 & 21.04 & 4.96 & 61.54 & 82.46 \\
\hline
\end{tabular}

Note: $N=18$. B-ADL $=$ Bayer Activities of Daily Functioning; Limit. $=$ limitations; Max. $=$ maximum score; Min. = minimum score; PHQ-9 = Patient Health Questionnaire; RAND SF-36 = Research and Development Short Form Health Survey; WHOQOL-OLD = World Health Organization Quality of Life for Older Adults.

${ }^{a}$ score of 5-9 indicates mild, 10-14 moderate, 15 and above severe depression (maximum score $=27$ ).

${ }^{\mathrm{b}} \mathrm{S}$ core of 1 indicates absence of difficulties in ADLs; higher scores indicate more difficulty.

${ }^{\mathrm{c}} N=8$ (administered only to spousal dyads).

${ }^{\mathrm{d}}$ Maximum score of 100 indicates highest level of functioning. ${ }^{\mathrm{e}} N=17$ (1 missing). 
Demographic variables. Spearman's correlations revealed that there was a relationship between older age and higher concern about dying (WHOQOL-OLD Death and Dying $r=-.49, p=.040$ ). In addition, higher educational level was associated with higher WHOQOL-OLD Intimacy scores $(r=.49, p=.048)$. Mann-Whitney test for independent samples did not identify any gender or marital status differences on selfreport measures. The results showed that retired individuals reported significantly higher level of depressive symptoms (PHQ-9 $U=6.00, z=-2.60, p=.009, r=-.63$ ), higher level of concern about dying (WHOQOL-OLD Death and Dying $U=11.00, z=-2.02$, $p=.048, r=-.49$ ), lower levels of energy (RAND SF-36 Energy/Fatigue $U=8.00$, $z=-2.33, p=.019, r=-.57$ ), and lower overall health (RAND SF-36 General Health $U=9.00, z=-2.23, p=.027, r=-.54)$.

\section{Post-Intervention Assessment}

Participants were re-assessed 7 to 24 weeks after the baseline assessment $(M=$ 14.7; $S D=6.21$ ). The following analyses were performed to address Hypothesis 3.1, which predicted lack of decline from pre- to post-test on any cognitive or self-report measures and possible gains on some test scores.

Cognitive measures. Table 7 presents descriptive statistics for cognitive test scores before and after the intervention and results of the Paired-Samples T-Tests $(N=$ 14). Assumption of equal variances was not violated. Significant improvements were found on the RBANS-U Visuospatial Domain $T(13)=-2.52, S E=3.61, p=.026,95 \% C I$ $[-16.86,-1.28])$, and on the Figure Recall subtest $T(13)=-4.11, S E=.43, p=.001,95 \%$ $C I[-2.72,-.85])$. Cohen's $d$ (correction for the correlation between pre- post-test scores) indicated large effect sizes (Cohen's $d=.67$ for Visuospatial domain and -1.10 for Figure 
Recall subtest). Due to multiple comparisons and the risk of Type I error, Bonferroni correction was performed by dividing the $p$-value of .05 by 19 (the number of test scores;

Field, 2013). The resulting $p$-value of .0026 was higher than the Visual Memory subtest $p$ $=.001$, but lower than the Visuospatial domain $p=.026$, suggesting that the significant change in the Visuospatial domain score might have occurred by chance. 
Table 7.

Descriptive Statistics for Cognitive Test Scores at Pre-Test and Post-Test and PairedSamples T-Tests.

\begin{tabular}{|c|c|c|c|c|c|c|}
\hline \multirow[t]{2}{*}{ Measure } & \multicolumn{2}{|c|}{$\underline{\text { Pre-Test }}$} & \multicolumn{2}{|c|}{$\underline{\text { Post-Test }}$} & \multirow[b]{2}{*}{$T(D F)$} & \multirow[b]{2}{*}{$p$} \\
\hline & $M$ & $S D$ & $M$ & $S D$ & & \\
\hline \multicolumn{7}{|l|}{ Stroop Color-Word Test ${ }^{\mathrm{a}}$} \\
\hline Word (word reading) & 10.07 & 2.20 & 10.21 & 3.04 & $-.29(13)$ & .78 \\
\hline Color (color naming) & 8.86 & 2.45 & 9.71 & 2.67 & $-1.67(13)$ & .12 \\
\hline Color-Word (word inhibition) & 10.71 & 3.27 & 10.43 & 3.63 & $.47(13)$ & .65 \\
\hline \multicolumn{7}{|l|}{ Trail Making Test ${ }^{\mathrm{a}}$} \\
\hline Part A (numbers only) & 10.79 & 2.52 & 10.07 & 3.36 & $1.10(13)$ & .29 \\
\hline Part $\mathrm{B}^{\mathrm{b}}$ (numbers and letters) & 11.36 & 2.59 & 10.92 & 3.10 & $.95(12)$ & .36 \\
\hline \multicolumn{7}{|l|}{ RBANS-U } \\
\hline Total Score ${ }^{c}$ & 98.14 & 14.26 & 101.64 & 13.48 & $-1.38(13)$ & .19 \\
\hline \multicolumn{7}{|l|}{ RBANS-U Domains': } \\
\hline Immediate Memory & 94.21 & 17.48 & 98.14 & 15.03 & $-.93(13)$ & .37 \\
\hline Visuospatial/Constructional & 91.93 & 19.17 & 101.00 & 18.66 & $-2.52(13)$ & $.026^{*}$ \\
\hline Language & 100.71 & 10.32 & 104.79 & 13.45 & $-.93(13)$ & .37 \\
\hline Attention & 108.79 & 15.13 & 104.71 & 14.14 & $1.23(13)$ & .24 \\
\hline Delayed Memory & 98.36 & 9.91 & 97.93 & 13.10 & $.12(13)$ & .90 \\
\hline \multicolumn{7}{|l|}{ RBANS-U Subtests ${ }^{\mathrm{d}}$ : } \\
\hline List Learning & 9.57 & 3.32 & 9.71 & 3.29 & $-.15(13)$ & .89 \\
\hline Story Memory & 8.43 & 3.16 & 9.57 & 2.85 & $-1.29(13)$ & .19 \\
\hline Figure Copy & 8.21 & 3.49 & 8.86 & 4.19 & $-.75(13)$ & .47 \\
\hline Semantic Fluency & 9.86 & 2.85 & 10.57 & 3.94 & $-.53(13)$ & .61 \\
\hline Digit Span & 11.29 & 2.49 & 10.71 & 3.58 & $.71(13)$ & .49 \\
\hline Coding & 11.50 & 3.25 & 10.64 & 3.20 & $1.43(13)$ & .18 \\
\hline Story Recall & 9.00 & 2.60 & 10.14 & 2.60 & $-1.61(13)$ & .13 \\
\hline Figure Recall & 8.93 & 2.40 & 10.71 & 2.34 & $-4.11(13)$ & $.001 *$ \\
\hline
\end{tabular}

Note. $N=14$. MMSE = Mini Mental State Examination; RBANS-U = Repeatable Battery for the Assessment of Neuropsychological Status-Updated.

${ }^{a}$ Age-corrected scaled scores based on population $M=10, S D=3$ (Mayo's Older Americans Normative Studies, Ivnik et al., 1996).

${ }^{\mathrm{b}} N=13$ (1 missing).

${ }^{c}$ Age-corrected index scores based on population $M=100, S D=15$ (publisher norms).

${ }^{\mathrm{d}}$ Age-corrected scaled scores based on population $M=10, S D=3$ (publisher norms).

$* \mathrm{p}<.05$ 
Figure 5 exhibits sample mean scores on five RBANS-U domains at pre- and post-intervention.

Figure 5

Pre-Test and Post-Test Means for Repeatable Battery for the Assessment of Neuropsychological Status-Update (RBANS-U).

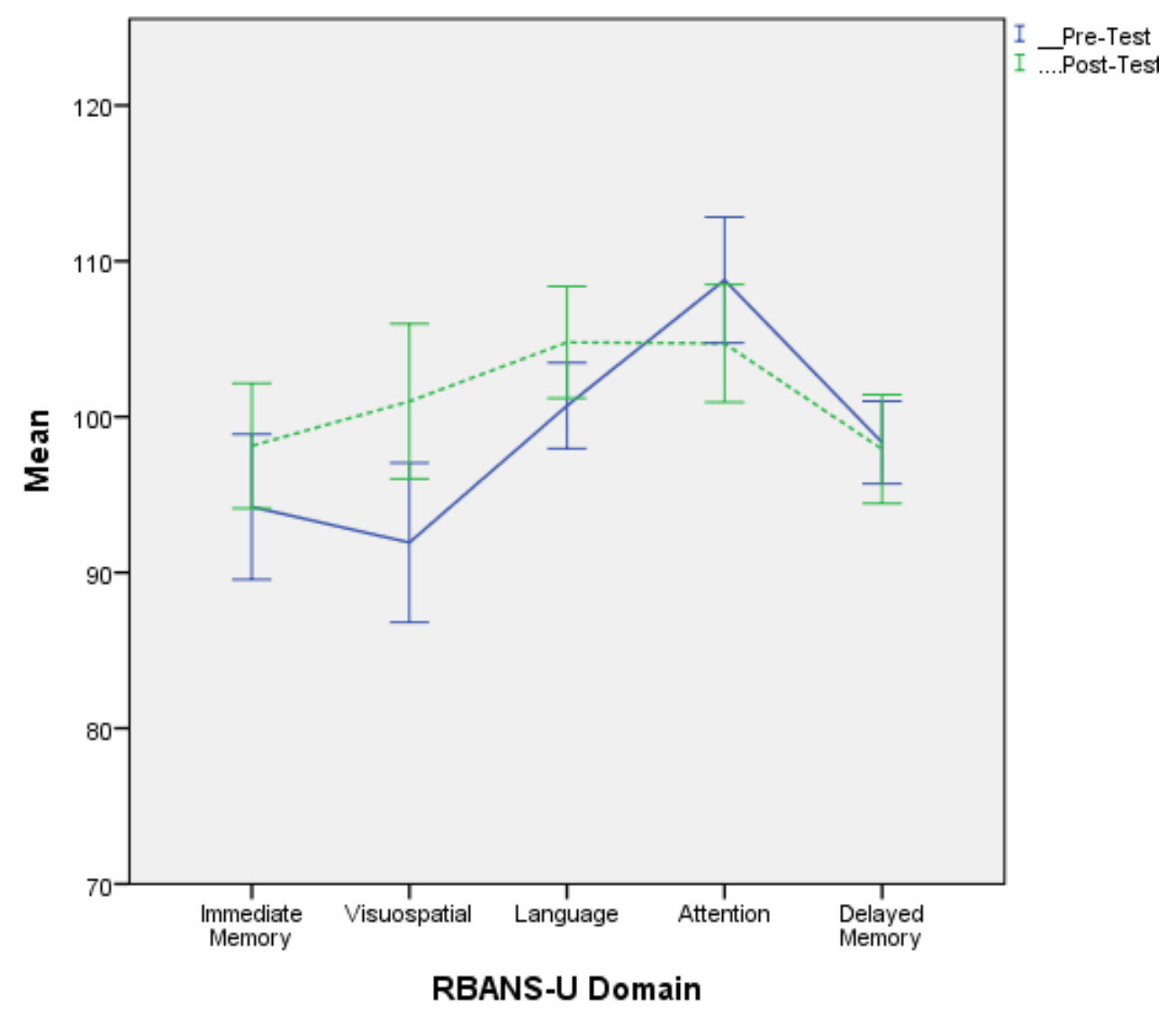

Error Bars: +/- $1 \mathrm{SE}$ 
Due to non-normality and mild outliers in score distributions for several cognitive measures, the same pre- and post-variables were analyzed using a nonparametric, Wilcoxon test for related samples. The results confirmed significant increase in the Visuospatial domain $(Z=-2.01, p=.044, r=.54)$ and the Figure Recall subtest scores $(Z$ $=-2.69, p=.007, r=.72$ ). However, these results were not significant when the corrected $p$-value of .0026 was applied to the results of the Wilcoxon test.

Demographic variables. Due to gender differences in several cognitive measures, mixed ANOVA was performed to evaluate the effect of gender on the pre-post score changes. Unless noted otherwise, the assumption of equality of covariances was not violated. The results of the analyses showed that there was no significant gender by time interaction for the Visuospatial domain (Wilk's Lambda $=.732, F(1,12)=4.39, p=.058$, partial eta squared $=.27)$. There was also no significant main effect for time $(F(1,12)=$ $4.39, p=.058$, partial eta squared $=.27$ ). However, there was a significant, large main effect for gender $(F(1,12)=10.08, p=.008$, partial eta squared $=.46)$. Same analysis was performed for the RBANS-U Figure Recall subtest. Again, the gender by time interaction was not significant (Wilk's Lambda $=.92, F(1,12)=1.01, p=.335$, partial eta squared $=$ $.08)$. However, there was a significant main effect for gender $(F(1,12)=7.44, p=.018$, partial eta squared $=.38)$ and for Figure Recall scores $(F(1,12)=13.35, p=.003$, partial eta squared $=.53$ ). As indicated by the partial eta squared values, the effect sizes for the two main effects were medium-to-large and large, respectively.

In addition to ANOVA, Independent-Samples T-Tests were performed for females and males separately. The results indicated that for females, significant improvement occurred on RBANS-U Visuospatial domain (pre-test $M=81.33, S D=$ 
12.30, post-test $M=94.44, S D=12.47 ; T(8)=-5.52, S E=2.38, p=.001,95 \% C I[-7.63$, -5.52], Cohen's $d=-1.84$ ) and on RBANS-U Figure Recall subtest (pre-test $M=7.78, S D$ $=1.48$, post-test $M=9.89, S D=1.97 ; T(8)=-3.92, S E=2.38, p=.004,95 \% C I[-3.35,-$ .87 , Cohen's $d=-1.35)$. Both effect sizes were large as indicated by Cohen's $\mathrm{d}>.80$. When Bonferroni correction was applied to the observed p-values, only the Visuospatial domain $p=.001$ was significant. Wilcoxon test for related samples confirmed significant improvements in females on the Visuospatial domain $(Z=-2.67, p=.008, r=.89)$ and the Figure Recall subtest scores $(Z=-2.40, p=.016, r=.80)$. Although the effect sizes represented by $r$ values were large, the corrected p-values were not significant.

For men, no differences from pre-test to post-test were found. These findings confirm that the participants' increase in scores on Visuospatial domain and delayed visual memory were attributable to improvement among females, but not males. Figures 6 and 7 illustrate the results for males and females on the RBANS-U Visuospatial domain and Figure Recall subtest. 
Figure 6.

Males and Females' Scores on the Visuospatial Domain of the Repeatable Battery for the Assessment of Neuropsychological Status-Update (RBANS-U).

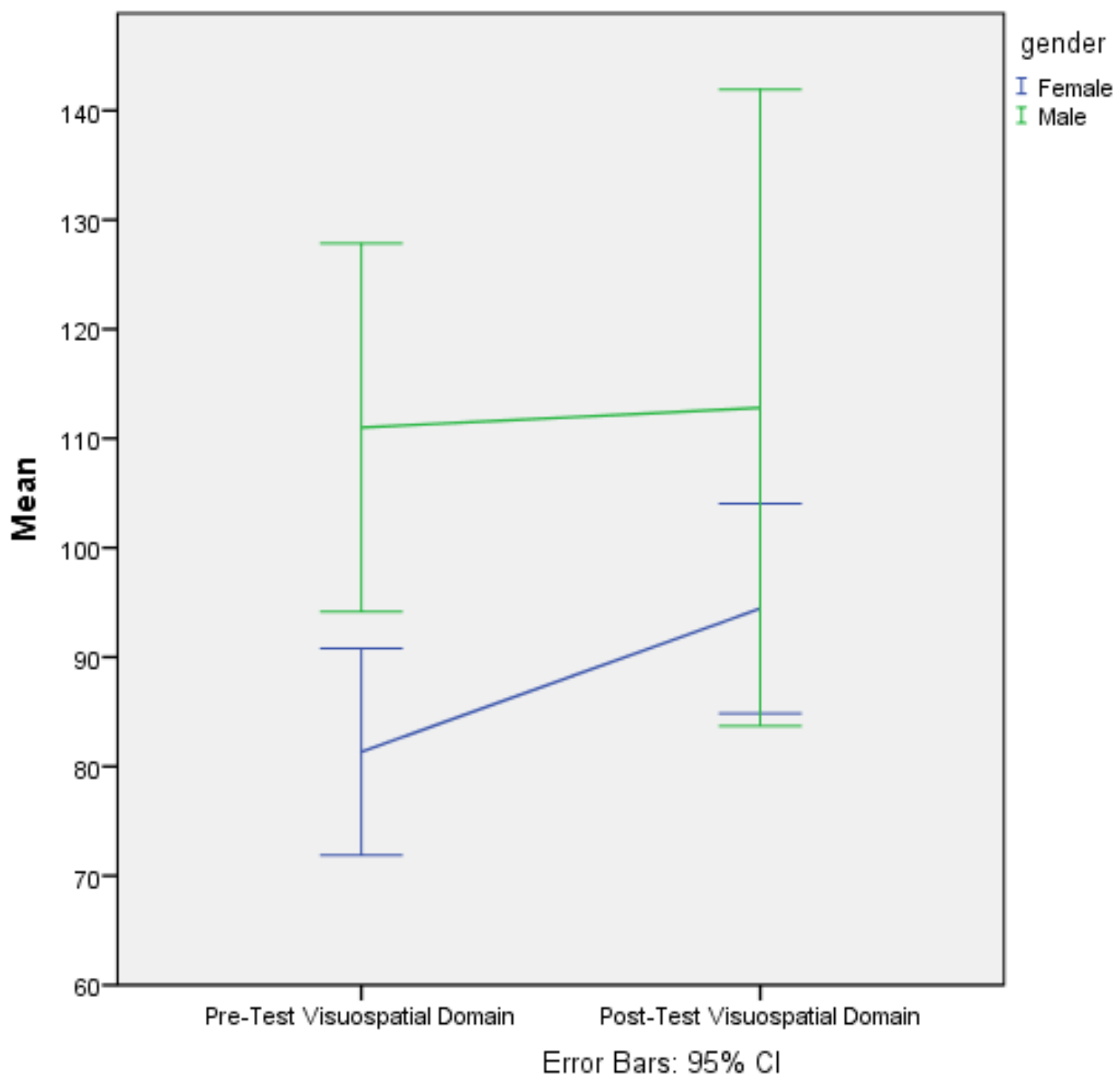


Figure 7.

Males and Females' Scores on the Figure Recall Subtest of the Repeatable Battery for the Assessment of Neuropsychological Status-Update (RBANS-U).

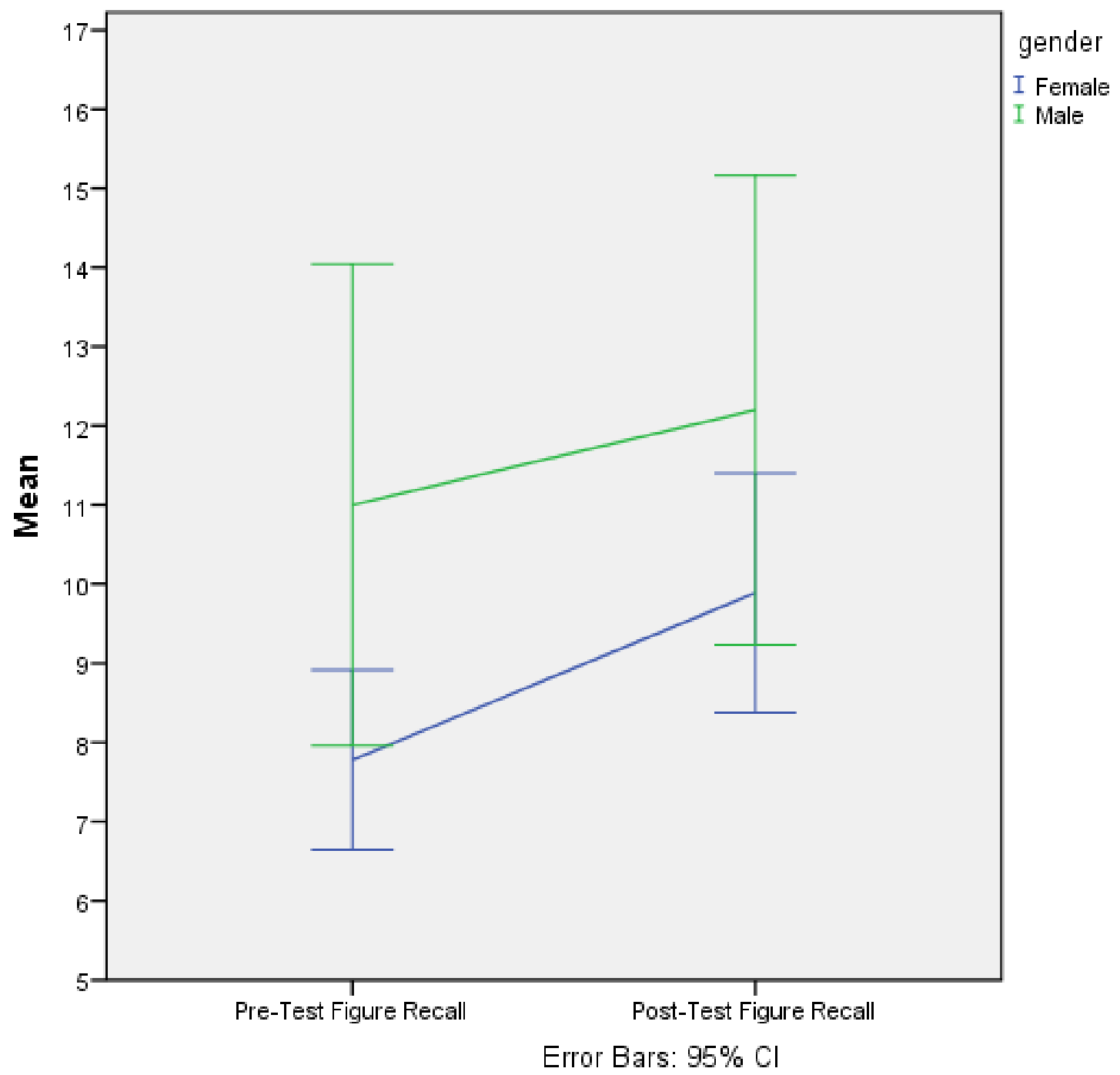

Due to the small sample size, it may be useful to present the data in terms of frequencies for exploratory purposes. Table 8 presents the number of participants who showed increase or decrease of one standard deviation or more or no change from pre- to 
post-test on each of the cognitive test scores (Stroop, TMT, \& RBANS-U subtests population $S D=3$; RBANS-U Total and Domain population $S D=15$ ). The majority of participants had stable scores on Stroop and TMT. A small, equal number of participants had increase and decrease in scores for Stroop Word and Stroop Color-Word scores. For Stroop Color, 4 (29\%) of participants had an increase and $1(7 \%)$ had a decrease in scores. For TMT A and TMT B, more participants showed a decrease in scores than increase ( 1 case increased on TMT A and B vs. 3 and 4 cases decreased, respectively).

As for the RBANS-U, it can be seen in Table 8 that overall, the scores were stable from baseline to the post-intervention assessment. Two participants improved by more than a standard deviation on the Total score (comprised of the five domain scores), and none of the participants exhibited a decline of more than a standard deviation. Moreover, half of the participants ( 7 cases) had an increase in their Visuospatial domain scores, and three had an increase on Immediate Memory and Language domains. Looking at the TBANS-U subtests, 4 (25\%) participants had an increase on Story Recall and 5 (36\%) had an increase on Figure Recall, and none of the participants demonstrated a decrease in these scores. However, more participants had a decrease in their scores on Digit Span and Coding (29\% on both) compared to those who improved. Four participants (29\%) had an increase in scores on List Learning, Figure Copy, and Semantic Fluency, and three (21\%) had an increase on Story Memory scores. On the other hand, the scores on List Learning decreased in five cases, four cases on Semantic Fluency, three cases on Figure Copy, and one on Story Memory. The four subtests that are scored as a percentile range were excluded from the frequency table. 
Table 8.

Numbers of Participants Who Demonstrated Increase, Decrease, or No Change on

Cognitive Test Scores.

\begin{tabular}{|c|c|c|c|}
\hline \multirow[t]{2}{*}{ Measure } & \multicolumn{3}{|c|}{ Change in scores $>1 S D[\#$ cases $(\%)]$} \\
\hline & Increase & Decrease & $<1 S D /$ no change \\
\hline \multicolumn{4}{|l|}{ Stroop Color-Word Test ${ }^{\mathrm{a}}$} \\
\hline Word (word reading) & $2(14 \%)$ & $2(14 \%)$ & $10(71 \%)$ \\
\hline Color (color naming) & $4(29 \%)$ & $1(7 \%)$ & $9(64 \%)$ \\
\hline Color-Word (word inhibition) & $1(7 \%)$ & $1(7 \%)$ & $12(86 \%)$ \\
\hline \multicolumn{4}{|l|}{ Trail Making Test } \\
\hline Part A (numbers only) & $1(7 \%)$ & $3(21 \%)$ & $10(71 \%)$ \\
\hline Part B (numbers and letters) ${ }^{\mathrm{a}}$ & $1(8 \%)$ & $2(15 \%)$ & $10(77 \%)$ \\
\hline \multicolumn{4}{|l|}{ RBANS-U } \\
\hline Total Score & $2(14 \%)$ & 0 & $12(86 \%)$ \\
\hline \multicolumn{4}{|l|}{ RBANS-U Domains: } \\
\hline Immediate Memory & $3(21 \%)$ & $3(21 \%)$ & $8(57 \%)$ \\
\hline Visuospatial/Constructional & $7(50 \%)$ & $1(7 \%)$ & $6(43 \%)$ \\
\hline Language & $3(21 \%)$ & $1(7 \%)$ & $10(71 \%)$ \\
\hline Attention & $1(7 \%)$ & $1(7 \%)$ & $12(86 \%)$ \\
\hline Delayed Memory & $1(7 \%)$ & $3(21 \%)$ & $10(71 \%)$ \\
\hline \multicolumn{4}{|l|}{ RBANS-U Subtests: } \\
\hline List Learning & $4(29 \%)$ & $5(36 \%)$ & $5(36 \%)$ \\
\hline Story Memory & $3(21 \%)$ & $1(7 \%)$ & $10(71 \%)$ \\
\hline Figure Copy & $4(29 \%)$ & $3(21 \%)$ & $7(50 \%)$ \\
\hline Semantic Fluency & $4(29 \%)$ & $4(29 \%)$ & $6(43 \%)$ \\
\hline Digit Span & 0 & $4(29 \%)$ & $10(71 \%)$ \\
\hline Coding & $1(7 \%)$ & $4(29 \%)$ & $9(64 \%)$ \\
\hline Story Recall & $4(29 \%)$ & 0 & $10(71 \%)$ \\
\hline Figure Recall & $5(36 \%)$ & 0 & $9(64 \%)$ \\
\hline
\end{tabular}

Note. $N=14$; RBANS-U = Repeatable Battery for the Assessment of Neuropsychological Status-Updated. ${ }^{\mathrm{a}} N=13$ (1 missing).

Table 9 presents another approach to the visual analysis of data. It provides demographic data, dyad type, number of cognitive training sessions, and changes in 
scores for each individual case. For RBANS-U, only domains scores are presented since they are comprised of individual subtest scores, four of which cannot be quantified in terms of SD change as they are scored as percentile range. It can be seen that although there were some decreases in scores, overall there were more increases in scores among the participants. On measures of executive functioning (Stroop and Trail Making Test), 43 percent of participants (6 cases) increased on at least one of five scores (four participants (28\%) improved on one score, one (7\%) on two scores, and one $(7 \%)$ on three out of five scores). On RBANS-U domain scores, the majority of participants (10 cases, $71 \%$ ) improved on at least one of five cognitive domains (6 participants (43\%) improved on one, 3 (21\%) improved on two, and 1 (7\%) improved on three out of five cognitive domains scores). 
Table 9.

Each Participant's Changes in Scores on Cognitive Measures.

\begin{tabular}{|c|c|c|c|c|c|c|c|c|c|c|c|c|c|c|c|}
\hline & & & & & & Number & & Stroop & $\underline{\mathrm{TI}}$ & & $\underline{\mathrm{RB}}$ & ANS & -U I & Dome & ains \\
\hline & Age & $\mathrm{M} / \mathrm{F}$ & Retired & Married & Dyad & Sessions & $\mathrm{W}$ & $\mathrm{C} \mathrm{CW}$ & A & B & IM & VS & LA & AT & DM \\
\hline 1 & 82 & $\mathrm{~F}$ & Yes & Yes & Friends & 12 & & + & - & - & & & & - & \\
\hline 2 & 81 & $\mathrm{~F}$ & Yes & No & Friends & 12 & + & & + & & - & + & - & & \\
\hline 3 & 65 & $\mathrm{M}$ & Yes & No & Friends & 24 & & & & & & & & & - \\
\hline 4 & 70 & M & No & No & Friends & 24 & & - & & & & - & + & & - \\
\hline 5 & 74 & $\mathrm{~F}$ & Yes & No & Unfam. & 9 & + & + & - & + & - & & + & + & \\
\hline 6 & 65 & $\mathrm{~F}$ & No & No & Unfam. & 9 & & & & & & + & + & & \\
\hline 7 & 70 & $\mathrm{~F}$ & Yes & No & Friends & 21 & & & & & & + & & & \\
\hline 8 & 73 & $\mathrm{~F}$ & Yes & No & Friends & 21 & & + & & & + & + & & & \\
\hline 9 & 83 & M & Yes & Yes & Spouses & 16 & & & & & + & + & & & + \\
\hline 10 & 87 & $\mathrm{~F}$ & Yes & Yes & Spouses & 16 & & + & - & & + & & & & \\
\hline 11 & 70 & $\mathrm{M}$ & No & Yes & Spouses & 13 & - & & & & - & & & & \\
\hline 12 & 67 & $\mathrm{~F}$ & No & Yes & Spouses & 13 & - & - & & - & & & & & \\
\hline 13 & 80 & $\mathrm{M}$ & Yes & Yes & Spouses & 11 & & + & & & & + & & & - \\
\hline 14 & 74 & $\mathrm{~F}$ & Yes & Yes & Spouses & 11 & & & & & & + & & & \\
\hline
\end{tabular}

Note. $\mathrm{A}=$ Trail Making Test part $\mathrm{A}, \mathrm{AT}=$ Attention, $\mathrm{B}=$ Trail Making Test part $\mathrm{B}, \mathrm{C}=$ Color naming, $\mathrm{CW}$ $=$ Color - Word, $\mathrm{DM}=$ Delayed Memory, $\mathrm{F}=$ female, $\mathrm{IM}=$ Immediate Memory, LA = Language, $\mathrm{M}=$ male, RBANS-U = Repeatable Battery for the Assessment of Neuropsychological Status-Update; TMT = Trail Making Test, $\mathrm{VS}=$ Visuospatial/Constructional, $\mathrm{W}=$ Word reading.

+ increase in scores by 1 standard deviation or more (Stroop, TMT $S D=3$; RBANS-U domains $S D=15$ ).

- decrease in scores by 1 standard deviation or more.

Self-report measures. Wilcoxon test for related samples was used to identify changes from pre- to post test on subjective measures. According to the results, there was a significant change on WHOQOL-OLD Death and Dying. The sample reported an increased level of concern about dying at post-test $(Z=-2.08, p=.037, r=.56)$, although the $p=$ value did not reach significance when Bonferroni adjustment was applied. 
Illustrative case studies. Due to the small number of participants, group statistical analyses may not adequately capture the true potential of the intervention. In addition, the sample was highly diverse in terms of age, employment status, marital status, and other variables, and two of the participants had neurological abnormalities (brain tumor and medication for cognitive decline). These factors impact internal validity and further complicate group analyses. Even though the focus of cognitive training is maintaining cognitive skills and promoting cognitive vitality (Ball et al., 2013), several participants demonstrated considerable gains in some cognitive skills, which points to the potential benefits of the novel intervention. Some of these cases are presented below. Table 10 includes functioning range classifications for scaled and domain scores, and Table 11 provides classifications for RBANS-U subtest percentile ranges (both adopted from the RBANS-U manual; Randolph, 2012).

Table 10.

Qualitative Descriptors for Scaled and Domain Scores.

\begin{tabular}{lll}
\hline Scaled Score & Domain Score & Range \\
\hline 16 and above & 130 and above & Very Superior \\
$14-15$ & $120-129$ & Superior \\
$12-13$ & $110-119$ & High Average \\
$8-11$ & $90-109$ & Normal/Average \\
$6-7$ & $80-89$ & Low Average \\
$4-5$ & $70-79$ & Borderline \\
3 and below & 69 and below & Extremely Low \\
\hline
\end{tabular}


Table 11.

Qualitative Descriptors for Repeatable Battery for the Assessment of

Neuropsychological Status (RBANS-U) Subtest Percentile Ranges.

\begin{tabular}{ll}
\hline Percentile & Range \\
\hline $14-15$ & Superior \\
Above 75 & High Average \\
$26-50,51-75$ & Normal/Average \\
$10-16,17-25$ & Low Average \\
$3-9$ & Borderline \\
2 and below & Extremely Low \\
\hline
\end{tabular}

Case A. Case A was a retired, divorced, 73-year old female whose native language was Spanish. B had 14 years of education (2 years of college), and was a part of the "friends" dyad. As shown in Table 12, A's executive functioning skills (Stroop and TMT) before and after the intervention fell in the normal range, with the exception of Stroop Naming, which increased borderline to normal range. A's overall cognitive functioning, represented by RBANS-U Total score was within the low average-to normal range and increased slightly by 8 points from before to after the intervention, which is not considered substantial. However, examination of her domain scores reveals that A's Immediate Memory increased from borderline to normal range (from $7^{\text {th }}$ to $42^{\text {nd }}$ percentile), and her Visuospatial skills increased from extremely low to low average (from $1^{\text {st }}$ to $14^{\text {th }}$ percentile). The other domain scores were normal and remained relatively stable from pre- to post-intervention. 
In terms of A's subtest scores, her pre-test score on List Learning (which assesses immediate verbal memory for unrelated pieces of information) fell within the lower portion of normal range, while her pre-test score on Story Memory (which assesses immediate narrative/episodic memory) was Borderline ( $2^{\text {nd }}$ percentile). At post-test, her List Learning score increased to high average $\left(84^{\text {th }}\right.$ percentile). Together with a nonsignificant increase in Story Memory from borderline to low average ( $2^{\text {nd }}$ to $9^{\text {th }}$ percentile), the improved List Learning score explains the dramatic gain in the overall Immediate Memory domain. Similarly, A's Line Orientation (which requires estimating distances or angles between lines) increased from $3-9^{\text {th }}$ (borderline) percentile range to $51-75^{\text {th }}$ percentile range (normal), leading to the improvement in the overall Visuospatial domain score.

Even though E's short-term memory for words (List Learning) improved, a decrease in her working memory from normal to low average was observed on Digit Span, which required her to repeat back strings of digits. At the same time, her delayed verbal memory for narrative information (Story Recall) increased from borderline to normal. Her delayed memory for a word list (List Recall) increased from $17-25^{\text {th }}$ to $51-$ $75^{\text {th }}$ percentile. The rest of subtest scores remained relatively stable. 
Table 12.

Case A: Pre-Test and Post-Test Cognitive Scores.

\begin{tabular}{|c|c|c|c|c|c|}
\hline \multirow[t]{2}{*}{ Measure } & \multicolumn{2}{|c|}{ Pre-Test } & \multicolumn{2}{|l|}{ Post-Test } & \multirow{2}{*}{$\begin{array}{c}\text { Score } \\
\text { Difference }\end{array}$} \\
\hline & Score (\%ile) & Range & Score (\%ile) $\mathrm{R}$ & Range & \\
\hline MMSE (30 points maximum) & $27(\mathrm{n} / \mathrm{a})$ & $\mathrm{N}$ & $28(\mathrm{n} / \mathrm{a})$ & $\mathrm{N}$ & 1 \\
\hline \multicolumn{6}{|l|}{ Stroop Color-Word Test ${ }^{\mathrm{a}}$} \\
\hline Word (word reading) & $10(41-59)$ & $\mathrm{N}$ & $10(41-59)$ & $\mathrm{N}$ & 0 \\
\hline Color (color naming) & $4(2)$ & BDL & $8(19-28)$ & $\mathrm{N}$ & $4^{*}$ \\
\hline Color-Word (word inhibition) & $10(41-59)$ & $\mathrm{N}$ & $9(72-81)$ & $\mathrm{N}$ & -1 \\
\hline \multicolumn{6}{|l|}{ Trail Making Test ${ }^{\mathrm{a}}$} \\
\hline Part A (numbers only) & $8(19-28)$ & $\mathrm{N}$ & $9(29-40)$ & $\mathrm{N}$ & 1 \\
\hline Part B (numbers and letters) & $8(19-28)$ & $\mathrm{N}$ & $10(41-59)$ & $\mathrm{N}$ & 2 \\
\hline \multicolumn{6}{|l|}{ RBANS-U } \\
\hline Total Score ${ }^{b}$ & $82(12)$ & LA & $90(25)$ & $\mathrm{N}$ & 8 \\
\hline \multicolumn{6}{|l|}{ Domain ${ }^{\mathrm{b}}$ : } \\
\hline Immediate Memory & $78(7)$ & BDL & $97(42)$ & $\mathrm{N}$ & $19 *$ \\
\hline Visuospatial/Constructional & $66(1)$ & EL & $84(14)$ & LA & $18^{*}$ \\
\hline Language & $96(39)$ & $\mathrm{N}$ & $92(30)$ & $\mathrm{N}$ & -4 \\
\hline Attention & $97(42)$ & $\mathrm{N}$ & $91(27)$ & $\mathrm{N}$ & -6 \\
\hline Delayed Memory & $95(37)$ & $\mathrm{N}$ & $101(53)$ & $\mathrm{N}$ & 6 \\
\hline \multicolumn{6}{|l|}{ Subtest ${ }^{\mathrm{c}}$ : } \\
\hline List Learning & $8(25)$ & $\mathrm{N}$ & $13(84)$ & HA & $5 *$ \\
\hline Story Memory & $4(2)$ & BDL & $6(9)$ & LA & 2 \\
\hline Figure Copy & $4(2)$ & BDL & $5(5)$ & BDL & 1 \\
\hline Line Orientation $^{\mathrm{d}}$ & n/a (3-9) & BDL & $\mathrm{n} / \mathrm{a}(51-75)$ & $\mathrm{N}$ & 2 \\
\hline Picture Naming $^{\mathrm{d}}$ & $\mathrm{n} / \mathrm{a}(26-50)$ & $\mathrm{N}$ & $\mathrm{n} / \mathrm{a}(51-75)$ & $\mathrm{N}$ & 1 \\
\hline Semantic Fluency & $8(25)$ & $\mathrm{N}$ & $7(16)$ & LA & -1 \\
\hline Digit Span & $10(50)$ & $\mathrm{N}$ & $7(16)$ & LA & $-3 *$ \\
\hline Coding & $9(37)$ & $\mathrm{N}$ & $10(50)$ & $\mathrm{N}$ & 1 \\
\hline List Recall $^{\mathrm{d}}$ & $\mathrm{n} / \mathrm{a}(17-25)$ & LA & $\mathrm{n} / \mathrm{a}(51-75)$ & $\mathrm{N}$ & 2 \\
\hline List Recognition $^{\mathrm{d}}$ & $\mathrm{n} / \mathrm{a}(26-50)$ & $\mathrm{N}$ & $\mathrm{n} / \mathrm{a}(51-75)$ & $\mathrm{N}$ & 1 \\
\hline Story Recall & $5(5)$ & BDL & $8(25)$ & $\mathrm{N}$ & $3 *$ \\
\hline Figure Recall & $10(50)$ & $\mathrm{N}$ & $10(50)$ & $\mathrm{N}$ & 0 \\
\hline
\end{tabular}

Note $. \mathrm{BDL}=$ Borderline; $\mathrm{EL}=$ Extremely Low $\mathrm{HA}=$ High Average; $\mathrm{LA}=$ Low Average; $\mathrm{MMSE}=$ Mini Mental State Examination; N = Normal; RBANS-U = Repeatable Battery for the Assessment of Neuropsychological Status-Updated; $\mathrm{S}=$ Superior; VS = Very Superior.

${ }^{a}$ Age-corrected scaled scores based on population $M=10, S D=3$ (Mayo's Older Americans Normative Studies, Ivnik et al., 1996). 


\footnotetext{
${ }^{\mathrm{b}}$ Age-corrected index scores based on population $M=100, S D=15$ (publisher norms). ${ }^{c}$ Age-corrected scaled scores based on population $M=10, S D=3$ (publisher norms).

${ }^{\mathrm{d}}$ Percentage range rather than scaled score is provided by publisher.

* score difference of 1 or more standard deviations

** score difference of 2 or more standard deviations
}

Case B. Case B was a retired, college-educated, native English-speaking, Caucasian female aged 74 who was a part of the unfamiliar dyad as she needed a study partner and was connected with another participant by the researcher. The participants in this dyad requested to shorten the duration of intervention as they were losing interest and felt that the tasks were becoming too repetitive. The dyad had 9 sessions of cognitive training. Table 13 presents B's pre- and post-intervention scores, percentiles, and qualitative descriptors (range of functioning). The table also includes score differences from pre- to post-intervention, where differences of 1 or more standard deviations (where applicable) are marked by an asterisk (*), and differences of 2 or more standard deviations are marked by double asterisk $(* *)$. Score differences that are less than 1 standard deviation are considered to be within expected limits, attributed to normal fluctuations due to situational and other factors (Randolph, 2012). For the four RBANSU subtests that are scored as percentile range, score difference in the table was arbitrarily recorded as $+/-1$ if the participant's result increased or decreased to the next percentile quarter (e.g., from 26-50 to 51-75 percentile), as $+/-2$ if his/her score increased or decreased to the percentile quarter after next (e.g. from $26-50$ to above 75 percentile), and as $+/-3$ if the result increased or decreased to the third percentile quarter (e.g., from below 25 to above 75 percentile) .

Based on her pre-test scores on Stroop and TMT, B's executive functioning is strong, mostly falling within the normal or high average range. At post-test, two of her 
Stroop scores (reading speed and naming speed) and one of her TMT scores (sequencing/multitasking) improved by one standard deviation, placing most of the Stroop and TMT scores in the superior and very superior range (at or above $90^{\text {th }}$ percentile).

B's overall cognitive functioning at pre-test, as measured by RBANS-U Total score, fell in the normal range (score of 101), nearly at the population mean of 100. However, this score was not representative of her cognition, as there was substantial scatter among her domain scores, ranging from a superior Immediate Memory score of 129 to a borderline Visuospatial/Constructional score of 72 , both of which represent her relative strength and weakness. The other domain scores (Language, Attention, and Delayed Memory) were normal and high average. At post-test, B's Language score increased dramatically from $39^{\text {th }}$ to $>99^{\text {th }}$ percentile, or by 38 points, which represents an increase of over two standard deviations, placing her in the very superior range. Her Attention score increased by over one standard deviation, improving from $79^{\text {th }}$ to $97^{\text {th }}$ percentile. Although under one standard deviation, her Visuospatial and Delayed Memory scores increased by 9 points. Decrease was observed in B's Immediate Memory score from $97^{\text {th }}$ to $73^{\text {rd }}$ percentile, placing her in the normal range. Despite decrease in Immediate Memory, improvements in other domains cumulatively contributed to B's RBANS-U Total score increasing by more than a standard deviation (from 101 to 117) and from normal to high average range.

Looking at individual subtests that factor into the five RBANS-U domains, B's scores on both subtests that form the Immediate Memory domain decreased by 1 or more standard deviations from superior to high average and normal range. A dramatic increase 
was observed on B's Semantic Fluency, which required her to name as many objects that belong to a category as possible. This score increased by over 3 standard deviations, and from lower end of normal range to very superior range $\left(>99^{\text {th }}\right.$ percentile $)$. Other subtest scores that improved significantly include Coding (a measure of attention and multitasking with eye-hand coordination), which increased from normal to high average range $\left(50^{\text {th }}\right.$ to $84^{\text {th }}$ percentile), and Figure Recall (a measure of visuospatial delayed memory), which increased from low average to normal range $\left(9^{\text {th }}\right.$ to $50^{\text {th }}$ percentile). 
Table 13.

Case B: Pre-Test and Post-Test Cognitive Scores.

\begin{tabular}{|c|c|c|c|c|c|}
\hline \multirow[t]{2}{*}{ Measure } & \multicolumn{2}{|c|}{ Pre-Test } & \multicolumn{2}{|c|}{$\underline{\text { Post-Test }}$} & \multirow{2}{*}{$\begin{array}{c}\text { Score } \\
\text { Difference }\end{array}$} \\
\hline & Score (\%ile) & Range & Score (\%ile) & Range & \\
\hline MMSE (30 points maximum) & $28(\mathrm{n} / \mathrm{a})$ & $\mathrm{N}$ & $29(n / a)$ & $\mathrm{N}$ & 1 \\
\hline \multicolumn{6}{|l|}{ Stroop Color-Word Test $\mathrm{t}^{\mathrm{a}}$} \\
\hline Word (word reading) & $13(82-89)$ & HA & $16(98)$ & VS & $3 *$ \\
\hline Color (color naming) & $11(60-71)$ & $\mathrm{N}$ & $14(90-94)$ & S & $3 *$ \\
\hline Color-Word (word inhibition) & $14(90-94)$ & HA & $14(90-94)$ & S & 0 \\
\hline \multicolumn{6}{|l|}{ Trail Making Test ${ }^{\mathrm{a}}$} \\
\hline Part A (numbers only) & $16(98)$ & $\mathrm{VS}$ & $17(99)$ & VS & 1 \\
\hline Part B (numbers and letters) & $14(90-94)$ & HA & $17(99)$ & VS & $3 *$ \\
\hline \multicolumn{6}{|l|}{ RBANS-U } \\
\hline Total Score ${ }^{\mathrm{e}}$ & $101(53)$ & $\mathrm{N}$ & $117(87)$ & HA & $16^{*}$ \\
\hline \multicolumn{6}{|l|}{ Domain ${ }^{\mathrm{e}}$ : } \\
\hline Immediate Memory & $129(97)$ & $S$ & $109(73)$ & $\mathrm{N}$ & $-20 *$ \\
\hline Visuospatial/Constructional & $72(3)$ & BDL & $81(10)$ & LA & 9 \\
\hline Language & $96(39)$ & $\mathrm{N}$ & $134(>99)$ & VS & $38 * *$ \\
\hline Attention & $112(79)$ & HA & $128(97)$ & $\mathrm{S}$ & $16^{*}$ \\
\hline Delayed Memory & $98(45)$ & $\mathrm{N}$ & $107(68)$ & $\mathrm{N}$ & 9 \\
\hline \multicolumn{6}{|l|}{ Subtest $^{\mathrm{d}}$ : } \\
\hline List Learning & $15(95)$ & $S$ & $12(75)$ & HA & $-3 *$ \\
\hline Story Memory & $15(95)$ & S & $11(63)$ & $\mathrm{N}$ & $-4 *$ \\
\hline Figure Copy & $5(5)$ & BDL & $5(5)$ & BDL & 0 \\
\hline Line Orientation $^{\mathrm{e}}$ & $\mathrm{n} / \mathrm{a}(17-25)$ & LA & $\mathrm{n} / \mathrm{a}(26-50)$ & $\mathrm{N}$ & 1 \\
\hline Picture Naming $^{\mathrm{e}}$ & $\mathrm{n} / \mathrm{a}(51-75)$ & $\mathrm{N}$ & $\mathrm{n} / \mathrm{a}(26-50)$ & $\mathrm{N}$ & -1 \\
\hline Semantic Fluency & $8(25)$ & $\mathrm{N}$ & $19(>99)$ & VS & $11^{* *}$ \\
\hline Digit Span & $14(91)$ & $\mathrm{S}$ & $16(98)$ & VS & 2 \\
\hline Coding & $10(50)$ & $\mathrm{N}$ & $13(84)$ & HA & $3 *$ \\
\hline List Recall ${ }^{\mathrm{e}}$ & $\mathrm{n} / \mathrm{a}(51-75)$ & $\mathrm{N}$ & $\mathrm{n} / \mathrm{a}(51-75)$ & $\mathrm{N}$ & 0 \\
\hline List Recognition ${ }^{\mathrm{e}}$ & $\mathrm{n} / \mathrm{a}(51-75)$ & $\mathrm{N}$ & $\mathrm{n} / \mathrm{a}(51-75)$ & $\mathrm{N}$ & 0 \\
\hline Story Recall & $12(75)$ & HA & $13(84)$ & HA & 1 \\
\hline Figure Recall & $6(9)$ & LA & $10(50)$ & $\mathrm{N}$ & $4^{*}$ \\
\hline
\end{tabular}

Note . $\mathrm{BDL}=$ Borderline $; \mathrm{HA}=$ High Average LA $=$ Low Average $;$ MMSE $=$ Mini Mental State Examination; $\mathrm{N}=$ Normal; RBANS-U = Repeatable Battery for the Assessment of Neuropsychological Status-Updated; $\mathrm{S}=$ Superior; VS = Very Superior.

${ }^{a}$ Age-corrected scaled scores based on population $M=10, S D=3$ (Mayo's Older Americans Normative Studies, Ivnik et al., 1996). 


\footnotetext{
${ }^{\mathrm{b}}$ Age-corrected index scores based on population $M=100, S D=15$ (publisher norms).

${ }^{c}$ Age-corrected scaled scores based on population $M=10, S D=3$ (publisher norms). $\mathrm{d}^{\mathrm{e} P e r c e n t a g e}$ range rather than scaled scores are provided by publisher.

* score difference of 1 or more standard deviations

** score difference of 2 or more standard deviations
}

\section{Post-Intervention Interviews}

Semi-structured interviews were employed to address Research Question 1, evaluating feasibility and participant acceptability of the dyadic format of cognitive training intervention. As mentioned earlier, one-one-one interviews took place immediately following post-intervention assessment and lasted 20 to 30 minutes. A total of 14 interviews were conducted. ATLAS.ti software was used for coding and thematic analysis. Questions addressed the participants' experience with the self-administered, dyadic format, scheduling, dosage (frequency and duration), intervention content, userfriendliness of the manual, and overall experience with participation in the study.

The interviews were coded freely without using previously created (a priori) codes. Thus, data processing and analysis was an on-going process. During the coding of the first interview, a number of codes emerged. With each consecutive interview, the same codes were applied or new codes were created as needed. This process was repeated until coding of all interviews was completed. In some cases it became clear that the code was too general and needed to be divided into several more specific codes. In other cases, the code was too narrow and it was combined with other narrow code(s) into a more general code. After several interviews it was possible to begin grouping the codes by topic or theme and create code families. Some of the codes directly corresponded to the questions asked during the interviews, while other codes and themes emerged based on participants' spontaneous reflections. Code families included Dyadic Format, Scheduling, 
Content, Dosage, Setting, Materials, Outcomes, Suggestions, etc. Examples of themes were "beginning of intervention vs. later," "enjoyable tasks," "frustrating tasks," "contributing to research," "helping the researcher," "doing something different," "being active in the community," "making time for sessions," etc. Due to the small sample size and potential risk of breaking confidentiality of individuals, participants' age and gender are not specified, and their dyad type and intervention setting are included only where necessary.

Dyadic format. All participants stated that the dyadic format worked well for them. Several participants said that having a study partner contributed to their motivation and adherence to the intervention, as one individual (friends dyad) put it, "it disciplined us." Another person (unfamiliar dyad) said, "the good thing about the partner ... [is that] it keeps the motivation. Some participants said that they would had been more distracted and less motivated if they engaged in cognitive training by themselves. One of them said, “I would enjoy it, yes, but it wouldn't be the same. I would be more distracted and not give the importance [to it]. Having the other person, you are more attentive." One of the older adults said that even though paper-and-pencil approach worked well, computerized program would also be acceptable: "I could go either way because I use the computer a lot, so it wouldn't bother me to do [it on] the computer, it didn't bother me doing [it] with paper and pencil. I fluctuate both ways." Along the same lines, another older adult shared, "I would be willing to do [training] from home and if I could have a little more flexibility with time, I would be willing to participate."

The interviews also pointed to increased social engagement. Several participants reported that the sessions gave them an opportunity to spend more time together, "gossip 
and visit," and that they "had fun." One of the older adults (unfamiliar dyad) shared, "I'm not a grandparent, so we talked about things that she does and she got ... a grandson, but we are both active seniors." Another participant (friends dyad" acknowledged that the sessions allowed the two study partners learn more about one another. None of the participants indicated that the sessions affected the relationship with their study partner in a negative way.

When asked about the experience of taking turns being the trainer, all participants stated that that there were no issues or problems, describing it as a "nice experience." One individual stated, "we had a great time, we had a wonderful time doing it." Another one of the participants who was retired teacher said, "by the time we both been through full years plus education, we both been students and we both been teachers, and we know how to take a test." Another individual (unfamiliar dyad) indicated that the dyadic format with turn-taking was comfortable: “we didn’t feel training or being trained any differently... we are seniors, we are not intimidated by anything... it was easy going, meaning it's not like we were trying to get a job."

However, one of the participants (friends dyad) stated that at the beginning, there was a sense of competition from the other partner, and another one (friends dyad) said that at the beginning, there was some anxiety about giving a correct answer. In both cases, the participants reported that these feelings dissipated shortly. Another older adult said that being the trainer, "it took an effort" not to help the study partner on a task: "it was difficult for me not to take charge, because when you're training you have to let them learn. And they learn by struggling and I don't like to see people struggle." 
Having an existing positive relationship appeared to contribute to enjoying the interactive component of the training. A participant that was a part of the "friends" dyad explained that participating together worked well because they "have been friends for year and years and years." An individual who was a part of the spousal dyad said that because their relationship was "good," the interaction during the sessions was positive as well. The participants who did not know each other before the study and were connected by the researcher both indicated that they worked well together and had a lot in common. One of them explained, "I think we have similar interests on several levels ... I don't know if that's good for your study, that we were very much the same in terms of how we worked. Which made it very nice."

Setting. Flexibility in location of the intervention appeared to be an important factor for the participants. For spouses, being able to have sessions at home, and for nonspouses, living near one another and having sessions at a place of mutual convenience contributed greatly to their ability and willingness to participate. For instance, several participants who were holding their cognitive training sessions at home stated that they probably would not have participated if they had to travel. As one older adult explained, "I don't think we would have done it if we had to get in the car and go some place." One of the participants who had sessions elsewhere said, "she was living here, I was living here, the center is right here... So it was easy for us. I thinking if people weren't that close together it would make it more difficult." The same participant appreciated being able to meet at a senior center and supported by staff: "we have the center which was wonderful, and ... the director was just always very gracious. They always have food and 
free stuff going... Sometimes if it was really busy here and got noisy, he would offer us the conference room."

Scheduling. Many older adults stated that finding the time that worked for both individuals in the dyad was not difficult. For several older adults, it was more challenging. One of them said, "we had to make an effort to do it. And just making an effort to find the time... it did not bother me that I did that, because I do a lot of different things, and it didn't bother me taking the time to do it." Another participant stated, "it was not finding the time, it was making the time to do it." One of the older adults (friends dyad) indicated that while at first it was more difficult to coordinate the times for sessions, "it's a question of changing priorities."

Frequency and duration. The majority of older adults stated that 1 hour two times a week for 3 months was a "good amount." Some dyads indicated that they would prefer sessions to be less frequent, once a week, which is what was more feasible for them: "the first one or two weeks we were fine with it, and then ... all of a sudden we realized that we weren't doing it. Except for maybe once a week. So once a week was what we were really handing." On the other hand, several older adults expressed regret with the end of the intervention and wished that there were more material to work on. As one older adult put it, "we already did it, and now what?... I'm hoping to do more in the future and I'll definitely call you." The same individual mentioned wishing that the sessions were more frequent: "at times I would have loved ... to have done it every other day." Another participant indicated that sessions could have been longer than an hour: 
Actually with some of the tests, we said "let's keep going." You said an hour, we could have done it for two hours. Because once you get going, then you realize the tricks you're playing and it's easier to do the next task because you remember. Two dyads requested to shorten the duration of the intervention to 9 and 12 weeks. During interviews, they explained that their motivation and excitement about the intervention decreased over time since the tasks became "repetitive" and "boring," and they were "turned off" and "questioning the value" of the tasks, as some tasks did not seem relevant to cognition (i.e. visual tasks, discussed below). One of these individuals noted, "12 weeks is a long time. It really is." According to another participant, "it was very much fun until we stopped, that's why we stopped. Because it was no longer fun and we both said ... that we didn't feel like coming." However, one of the older adults who ended intervention early stated that "the second set of materials held our interest far more than the first set, and have we started with it, we might have continued." Another participant who asked to shorten the intervention mentioned that the two of them were busy and tired the day they decided to discontinue the sessions, which might have contributed to this decision: "if we had canceled and said let's do it next Tuesday, maybe we would have gone on.”

Materials. The majority of older adults stated that using the manual was easy and self-explanatory. Also, a number of older adults indicated that as time went by and they became familiar with tasks, they did not need to read the instructions as they knew what was involved. According to one individual, "one we got about midway, we knew what the pattern was, the exercises." Many participants mentioned that there were some typos and mistakes in tasks that needed to be corrected. For example, one individual said, 
"sometimes we found a typo or something that was ambiguous and we marked it on the pages." A few individuals mentioned that on rare occasions, the instructions were not clear. One of them said, "it was kind of hard sometimes to understand what they really wanted us to do." Also, two participants mentioned that some of the words in tasks were outdated. Further, one participant said that there should be a way to keep the used work sheets in the book, as it was difficult to fit them into the binder sleeve (the participants were asked to keep the work sheets with the binder). The participants also said that they did not have any difficulty using the log sheet, which is included at the beginning of the binder.

With regard to the sand clock, the majority of older adults liked using it and being able to see whether the 30 minutes have passed easily. However, in cases where the dyads did not meet at home, sometimes the sand clock would fall on the side and the participants had to wait for it to finish running or use their cell phone for timing. One person shared, "I liked the old fashioned [sand clock]... and I guess you have to let it settle before we started. But I liked it, ... I prefer that to electronic [timers]. Similarly, this individual's study partner said: "I used my timer on my phone too. It wasn't a problem, but it will tilt over if it's sliding around in your car."

Content. Some participants expressed very positive views about the tasks, stating that many exercises were "stimulating," and "challenging," although some of the tasks were "too easy" and "repetitive." One of the individuals reported feeling "energized" after the initial sessions, comparing mental exercise to physical exercise: “it's like jogging your brain.. so we were really energized, and that's a good feeling. Anything that can do that, and I'm used to doing it ... with physical fitness. So it's nice to have that 
mental challenge also." This participant and several others reported that as time went by, their enthusiasm diminished due to lack of challenge, excessive repetition, or because some tasks seemed irrelevant to cognition. According to one of them, "at the beginning it was fun, challenging, but after a while it got repetitive and we got bored with it." Another individual said, "it started as a real challenge [and we] had very high interest level, I think, and we worked well together." However, a few participants did not find the tasks redundant. One of them noted, 'I didn't find any of it, it never got redundant, even though you're doing the same tests over and over again, it never got redundant."

Some individuals stated that although they noticed an increase in difficulty, they wished there was more of an increase. The majority of older adults stated that the variety of tasks was appropriate. A number of participants indicated being aware of their cognitive strengths and weaknesses in terms of visual vs. verbal tasks, and one participant reported enjoying tasks that were easier for that person. As one older adult shared, "I found myself challenged on the visual things and probably gave up a little bit too easy." Another older adult elaborated on her strategy when approaching tasks: "how do I beat this test? What's the pattern there, what do I look for, how can I work my way through it?"

Several participants indicated that with time, they were doing better on tasks. One of them noted, "I felt like I was ... growing." Another participant recalled, "at the beginning we didn't have enough time, and in the end we were going faster and faster." Another individual said, "with repeating the numbers, I found in the beginning it would take me 2 or 3 sets of numbers to warm up, and I got better at it as we did the individual exercises and cumulatively I got better.” Interestingly, some participants said that 
sometimes the material triggered further exploration of the subject and learning new information, "we looked up tea, or herb, or spice or whatever, we learned a few things." Also, several participants reported that the material offered them a new perspective on things, "one of us would say to the other one- oh, I never would have thought of that."

In terms of the specific tasks, several individuals indicated that tasks where they listen to a story and were required to answer questions about it were "challenging." As one older adult said, "reading a story, we both had trouble with these. But we got better as we went along." Also, many individuals had difficulty with a deduction task where they had to use clues to identify characteristics of several persons. The participants were instructed to skip the tasks that were too frustrating or took too long, and several dyads skipped the deduction tasks. However, one participant stated that this was the most enjoyable task and expressed regret that these tasks were not included in the second binder of exercises: "[study partner] didn’t like them at all. I loved them and I was sorry that in the second book there were none." Some older adults mentioned enjoying language tasks (word coding, filling the blanks, stating different meanings of the same words, etc. ).

A few individuals thought that some visual attention tasks targeting visual acuity rather than cognition, as one put it "some exercises were more like a visual test, like I would be doing this in the ophthalmologist office rather than for brain training, so the purpose of that was elusive to us." Most of the participants found a visual attention task that provided a key word and required finding the letters among a string of letters that also appear in the key word to be "tedious," and "repetitive." As one person explained, "we both had to get out our glasses. The print was very close together, very jammed 
together, and I presume for pattern recognition." On the other hand, one of the participants stated enjoying this exercise.

Some participants did not like open-ended questions because "there was no right or wrong," because they "wanted some closure," or because the questions were too simple or unusual. Several others indicated enjoying the discussion. Examples of openended questions included identifying differences and similarities among objects belonging to the same category, imagining the same situation through the eyes of different characters, or thinking of ways to improve something (air travel, organized religion, etc.). Several other participants said that they "had fun" with those tasks.

One of the older adults indicated that their dyad expected the training to focus on memory and not other cognitive skills: "entirely my fault, I was thinking it would be more memory training than brain training. And I'm still not entirely clear about brain training, in the sense that for a normal healthy person, what do you train?" This participant felt that some of the exercises were more appropriate for cognitive rehabilitation, although the second binder of tasks "was far more interesting...far more challenging. Repetitive, but still more challenging." On the other hand, another participant reported enjoying the first binder more than the second: "The first book had more puzzles than the second book. The second book was way too easy."

Adherence. With regard to adherence, the participants reported that they were generally not distracted and stayed on tasks during the sessions. One of the older adults (friends dyad] shared that "when the hourglass was running we didn't gossip. I mean, we worked for the half an hour and half an hour, and then the timer ran out, we put it flat, we chatted, then we turned it over and started [again]... so we were very good about using 
the 30-minute ... segments." Another older adult said, "we were very disciplined because it wasn't a social visit, so we stayed pretty much on point. Made a comment or two, but that was it." Sometimes the participants continued working on exercises after the sand clock stopped running. One individual admitted, "we lost track of time a few times." Another participant said, "we had to remember sometimes to turn the timer over." One of the participant expressed concern about others' adherence, saying that "of course we didn't do that, but ... we imagined that some pairs would waste some of the time, you know if no one is supervising them... You have no idea whether or not we are giving each other hints or telling each other the answer, or leading us through the answer."

Perceived outcomes. With the exception of a few individuals who felt that their cognition improved, the participants said that they did not notice changes in their cognition or how they approach daily cognitive tasks over the course or after the intervention. When asked about any changes, one participant stated, "That's hard to say. I cannot say for sure that I am aware of. The things that were problems before, like where I left my glasses, still exist." Another older adult shared, "I don’t know what the results are one way or another, but I think it's great that you are doing that type of thing and turning it into this kind of a project." Several individuals expressed high interest in their cognitive test scores. According to one person, "I would be curious to see [the scores], I don't think I improved off the top of my head."

However, a number of individuals noted being more aware of the need to pay attention. One older adult noted, I always tell people, “you look at things but you didn’t see it." And I actually end up getting my own advice thrown back at me because of this program. Another individual said, "I make a point and insist on focusing," and another 
one stated, "the idea of paying attention is, we had to pay attention to some of the exercises, you had to listen to the story and try to remember details. That is, I think, the problem we have." Along the same lines, another individual said, "I think we both, we now both listen, it's not the proper word to use, but "harder", more attentively."

Some of the participants brought up examples of how being mindful about paying attention translated to other areas of life. One of them talked about checking her accuracy at work more carefully: "I'm verifying more what I'm doing." Another older adults said:

When I went to art basel I paid more attention to this kind of painting. I'm looking at it and I'm going, "why did they do that?" But I went and I spent more time, I didn't go looking for what I like. I went to see more, maybe to feel what somebody else is feeling.

Suggestions for improvement. The participants expressed that the format, setting, and the materials of the intervention were appropriate. The most frequent comments regarding improving the program were related to the content, as many older adults stated that the tasks should be more challenging, less repetitive, and with higher increase in difficulty. As an example, one participant suggested "more variety and maybe a little more increased difficulty, because if you can respond and get everything correct, that's not really challenging." Also, one older adult suggested that although some tasks were tedious and time-consuming, "I wouldn't eliminate any of them."

Another individual who enjoyed the deduction table task talked about enjoying the challenge:

When people are complaining about the box squares or that's too many numbers to repeat backwards or forwards, that's good. Those are the exercises that I want. 
I don't want exercises where I can repeat 8 numbers forward. Let's go backwards. I'd be lucky to get $4 \ldots$ So I think you gotta make it harder.

Another person suggested having different tasks with the level of difficulty based on each individual's level of cognition: "like a power test, go as far as you can go... go by level of difficulty level." One individual said that it would have been helpful to see an explanation for each task in terms of what specific cognitive function it is meant to train, since this was not obvious for some of the tasks. A few individuals also mentioned addressing some misspelling and semantic errors and clarifying some of the instructions in the manual. One of the older adults said that the frequency should be reduced to once a week, and another one stated that it should be shorter than 3 months.

The participants also had ideas about potential application of the intervention. One of the participants suggested that the program can be turned into a board game for older adults. Another one stated that the researcher should bring the intervention to larger institutions, and that dyadic approach can be useful in the Veterans Administration setting, with veterans supporting one another and helping each other with rehabilitation and reintegration, in the light of lack of medical staff. This individual had an analogy: "I might not know how to change a flat tire and be a mechanic, but at $2 \mathrm{am}$ I might be driving by [researcher] who's got two flat tires, and I don't know what I'd do about the second, but at least I can, or maybe we can do it together."

Overall experience. In general, the sample indicated that they enjoyed participating in the study, found it to be "interesting" and "informative," and that they "were glad to do it." One individual stated, “I didn't mind doing this at all. I don't see how anybody would." When asked whether "the program was a good fit for someone like 
you," all of the participants stated that it was. One older adult also reported increased confidence: "I really enjoyed it, it has given me confidence in myself. I really think [that] by testing myself and analyzing, I'm in better shape with my memory than I thought, so my self-esteem is up." Another person stated that it was "fun to do something a little bit different ... it allowed me to think a little bit out of the box and do things a little bit differently." Another participant said, "I like the overall concept of it because I think it's a really good thing to do." Further, one individual stated, "I found it interesting and I'd like to know where this exercise would come out, and I see a great possibility for, like a saying "people helping people".

It appeared the majority of the participants who decided to take part in the study despite lack of reimbursement, were acutely aware of the need to stay engaged and occupied, and motivated to enrich their lives with the Brain Training opportunity. These older adults appreciated the importance of cognitive stimulation and mental challenge, particularly in older age, and the need to push oneself. One older adult talked about noticing that some peers become disengaged and do not keep up with technology. Another one observed:

I don't seem to finish things. So I have to work at it and it could be that you live alone, it could be because you get old and "grudgedy," and that's why I do a lot of volunteer work. I'm always helping somebody. I don't do it for the money, I do it because I can. And I do get a lot of satisfaction out of problem-solving and fixing things. And there will come a time with every human being when we can't balance a check book and we can't get out of a bath tub. Because we're living longer. 
Some individuals also said that they valued the opportunity to contribute to research and to the researcher's scholarly pursuits. As one older adult said, “overall I'm more curious about your academic pursuit with this than I was with my own participation." Another person stated, "[it was] fun to do something different and to see a way of helping you out in your work because of the fact that hopefully it will help me in the long run." Also, several older adults said that they were interested in finding out about the methodology and the results of the study. One interviewee said, "I'm curious about the sample, ... about the validity, ... and what you'll do with it later." 


\section{DISCUSSION}

The study involved evaluation of a novel approach to paper-and-pencil cognitive training, which employed self-administered, dyadic intervention with coaching, combining the benefits of cognitive and social engagement. Because this approach has not been investigated before, the focus of the study was to explore the participants' experiences with the cognitive training program and determine whether the dyadic approach was feasible and whether the older adults were receptive to the intervention. The main goal was to establish whether the intervention may be beneficial in cognitively healthy older population and should be evaluated in larger scale studies. Lastly, the study aimed to point to the appropriate setting, dosage, and materials for dyadic cognitive training intervention in future studies.

Even though the study included a pre- and post-intervention assessment, the design of the study did not allow drawing firm conclusions with regard to the efficacy of the program since the focus was on feasibility, a necessary step preceding efficacy testing in subsequent RCTs. For this reason, external validity/generalizability was the priority in the study, and the design was characterized by broad inclusion criteria, small sample size, and absence of a control group. Overall, the results of the study were promising and showed that the self-administered, dyadic approach with coaching was well-received by the participants who appreciated flexibility and accessibility of the program. Participant interviews provided useful information with regard to their experience with the program and suggestions on how it can be improved. The study also shed light on numerous recruitment, retention and adherence, materials, and other methodological factors which may be relevant in future research. These findings are discussed below. 


\section{Recruitment}

Recruitment of participants was more difficult and took longer than anticipated. The time of recruitment (summer) contributed to this issue, since many English-speaking older adults ("snowbirds") reside in the North-East during the summer months, and come back to Florida during the winter months. Also, many older adults travel during the summer. Limiting the participants to English-speaking individuals created another barrier to recruitment, as many older adults in South Florida are Spanish-speaking. These issues contributed to the fact that the sample was not representative, as the majority were Caucasian older adults with college or graduate-level education and likely higher income. Going forward, it is important to adapt the materials in Spanish and other languages and use translated versions of assessment tools which have been standardized in other languages and populations.

With regard to the recruitment sources, the study demonstrated that a number of recruitment strategies were effective, ranging from flyers placed in public libraries, senior centers, and coffee shops to CraigsList advertisements, snowball recruitment, and attendance of psychoeducational presentations. The fact that other strategies (flyers in low-income senior housing, community health centers, YMCAs, etc.) produced some contacts but did not lead to participant enrollment points to the self-selection bias in the study. It is possible that older adults who visit libraries, attend senior center activities, use the internet, and attend psychoeducational events are more likely to be educated and motivated to stay active and informed, promote their cognitive vitality and overall wellbeing, and take part in research despite the lack of reimbursement. 
It should be noted that many public locations (public parks, recreation facilities, etc.), low-income senior housing buildings, and other residential buildings do not allow flyers being posted on their message boards. Despite the non-profit nature of the study, administrators of many locations viewed the flyers as advertisement, and did not approve for them to be posted. This limited the number of places where the flyers could be placed, and might have contributed to the skewness of the sample. Making personal connections with program directors and other managerial staff via email or in-person proved to greatly improve the chances of successful recruitment.

Another unanticipated finding during recruitment was that more non-spousal dyads enrolled in the study and began the intervention compared to participants who enrolled with their spouse. Six dyads included friends, neighbors, and unfamiliar older adults, and five dyads were married couples. Additionally, one of the participants was married and resided with her spouse, but chose to participate in the program with her friend. These findings supports the idea that limiting dyadic cognitive training to couples is inappropriate, as it would lead to exclusion of many single, widowed, divorced, separated, and even married older adults interested in participating with another familiar or unfamiliar older adult.

Notably, a number of older adults interested in participating in the study did not enroll due to not having a study partner. This was one of the main reasons for not enrolling, which was an unexpected finding. Several of these individuals inquired whether they could be connected with another potential participant in need of a study partner, which was successfully carried out for one of the dyads. This demonstrates that many older adults are interested in promoting their cognitive functioning and are willing 
to engage in dyadic training even with an unfamiliar person. This also suggests that potentially, dyadic cognitive training can be offered as a class in community settings where older adults can team up with both familiar and unfamiliar peers. Moreover, in such programs older adults may pair up with different study partners during each session, which further increases flexibility of the dyadic, paper-and-pencil approach.

\section{Retention}

Originally, a total of 11 dyads enrolled in the study and underwent preintervention assessment. Two of the dyads withdrew from the study. In one case, the two older adults were unfamiliar seniors connected by the researcher, and one of them expressed concern about their age difference, difference in retirement status, and residing too far from one another. One of these older adults was driving, and the other one had access to transportation services free of charge. Nevertheless, one of the older adults requested to be paired up with someone who is also retired and lives closer. This issue pointed to the importance of study partners living close to one another, regardless of whether they are familiar or unfamiliar older adults. In case of unfamiliar dyads, future studies need to take into account geographic factor when connecting older adults. Participant interviews confirmed this conclusion, as many of the respondents stated that not having to travel was among the most important characteristic of the intervention. Also, both participants in the unfamiliar dyad said that the convenience of meeting at the senior center, which was within walking distance for both of them, contributed greatly to their ability and willingness to participate in the study.

The other dyad that withdrew immediately after the pre-intervention included two neighbors living in the same building. One of them was a native English speaker, and the 
other older adult was Spanish-speaking. The Spanish speaking individual changed her mind about participating due to health problems and concern about the language barrier. Again, this points to the importance of a good fit among the two participants in terms of language and health status, in addition to the employment status and residential location.

During the course of the intervention, two dyads dropped out of the study. In one case (neighbors dyad), one of the participants passed away. In the other case (spousal dyad), one of the participants had serious health issues. This represents an acceptable drop-out rate of $22 \%$, which is relatively close to the hypothesized attrition rate of $20 \%$ (Research Question 1, "What are the participants' retention and adherence rates?"; Hypothesis 1.1). In comparison to this rate, other cognitive training studies had attrition rates ranging from 2 to 48 percent, with higher attrition among computerized interventions.

\section{Adherence}

It was hypothesized that on average, the participants would complete 80 percent (19 out of 24) sessions. In the study, the adherence rate was 15.14 sessions, suggesting that Hypothesis 1.2 should be rejected. However, participants' adherence rate was affected by the fact that two dyads requested to shorten the intervention to 9 and 12 sessions because they felt that many tasks were too easy and the tasks were getting repetitive. Also, three dyads completed all tasks within fewer sessions $(13,16$, and 21 sessions). The only dyad that completed 24 sessions decided to shorten the sessions toward the end of the intervention to ensure that they had enough tasks to stretch over the remainder of 24 sessions. Another factor that affected adherence was that for many 
dyads, intervention fell on Thanksgiving and Christmas holidays, which affected their ability to schedule the cognitive training sessions during that time.

The variability in frequency and duration of the intervention among the participants points to several conclusions. First, 24 sessions over 12 weeks maybe an overly long duration for such an intervention, particularly when the participants are unable to meet twice a week. Second, there is a need to include more challenging tasks in the intervention to ensure that the participants' enthusiasm and motivation do not diminish. Third, the variability in the number of sessions it took to complete all tasks demonstrates that some older adults are much faster than others, so again, more tasks of more challenging nature need to be included in the manual. Based on their interviews, the participants stayed on tasks and worked on the material during the sessions, with an exception of occasional comments. Not having a facilitator did not seem to affect the participants' compliance with the intervention.

\section{Quantitative Findings}

Since the study focused on feasibility, the results of the statistical analyses should be interpreted with caution. Specifically, the interpretation of results is complicated by a lack of a control group, violations of assumptions in several cognitive and the majority of self-report variables, and variability in the participants' demographic characteristics and the number of sessions completed. Also, the number of individuals who completed the intervention and post-intervention assessment $(N=14)$ was lower than the minimum number of 20 required for the power of .80 , as indicated by the a-priori power analysis.

It should also be noted that due to difficulty with recruitment, inclusion criteria were expanded to include several participants who underwent cognitive testing as part of 
medical research within the previous several years, and one participant on a daily cognition-enhancing medication (in the absence of MCI or dementia diagnosis). Additionally, one of the participants was diagnosed with a brain tumor shortly after completing the post-intervention assessment. Although these factors complicate the interpretation of the results, they illustrate the fact that older adult population is highly multifaceted and diverse, and there is a need to utilize less stringent criteria when it comes to older adults in research in order to reduce barriers to participation and increase representativeness of the sample, as discussed by Mody et al. (2008).

Pre- and post-test cognitive measures included MMSE, Stroop Color-Word Test, TMT, and comprehensive neuropsychological battery RBANS-U (two different forms of RBANS-U were administered to participants at pre- and post-intervention assessment to reduce potential practice effects). Of note, Stroop and TMT population age norms were based on three-year increments (i.e., 64-66 years, 67-69 year, etc.; Ivnik et al., 1996). In contrast, RBANS-U publisher norms are based on ten-year increments (i.e., 60-69 year, 70-79 years, etc.; Randolph, 2012). As a result, these norms may not adequately differentiate between individuals of various ages or capture the subtle cognitive decline that occurs over the course of 10 years in older adults. For example, two individuals aged 70 and 79 are both compared to the same population mean, which may result in inflated scores for the younger individual and lower scores for the older individual.

MMSE and PHQ-9 were a part of the screening procedure and post-test assessment. Self-report measures assessed depression (PHQ-9), quality of life (WHOQOL-OLD), health status (RAND SF-36), and daily functioning/IADLs (B-ADL). Both parametric and non-parametric tests were utilized in the study. Parametric tests were 
supplied by non-parametric tests due to the small sample size and violation of normality assumption for several cognitive variables. Non-parametric statistics were utilized for self-report measures, since the vast majority of these variables were not normally distributed.

Despite the relatively high educational level among the participants, the sample demonstrated average cognitive functioning which points to the generalizability of the sample with regard to cognition. The exceptions were scores on a measure of attention, processing, and multitasking, which were significantly higher than the population mean, and measures of visuospatial skills and delayed visual memory, which were lower than the population mean. As a group, the participants' attention appeared to be a relative strength compared to other cognitive functions.

Hypothesis 4.1 (association of education, age, and health status with pre-test cognitive scores; no association with gender) was partially rejected. Educational level was not associated with scores on any cognitive measures, while age was positively correlated with language skills only. As for the health status, lower executive control was linked with higher levels of pain, and weaker language skills were related to lower energy, sensory functioning, and overall health. Poor visuospatial skills were also associated with lower sensory functioning. When males and females were compared on cognitive test scores, males outperformed females on measures of visuospatial reasoning and delayed visual memory, suggesting that significant deviations from the population norm on these measures were attributable to the lower performance among female participants. 
For self-report measures, the sample scored around 75 and higher (maximum score $=100)$ on the majority of quality of life and health status domains, but reported lower energy (median score of 66). The participants reported little difficulty in IADLs. No gender differences were found on self-report scores. Interestingly, older age was linked with concern about dying only, while retired participants had significantly higher level of depressive symptoms, lower energy, and lower overall health, in addition to higher concern about dying. This suggests that retirement status has a stronger relationship with perceived health and well-being compared to age. Since age was linked with language skills and married and retired participants were older, it is not surprising that they scored higher on language skills compared to those employed and not married. Married individuals also tended to be more educated.

The four individuals who dropped out during the course of the intervention did not differ from the rest of the sample, with the exception of lower attention scores and lower self-reported health scores. Higher number of sessions completed and the duration of intervention were associated with lower scores on several cognitive and self-report measures. It is possible that individuals with weaker cognitive skills and lower levels of health and quality of life took longer to complete the tasks. It is also possible that these individuals were more concerned about their cognition or more aware of their cognitive weaknesses and therefore, were more motivated to complete the program in its entirety.

The results of pre- to post-intervention analyses addressed Research Question 3, change in cognitive and self-report scores from pre- to post-intervention. As previously, discussed, the aim of cognitive interventions is to address cognitive decline by delaying its onset and/or slowing its progression, which takes longer period of time and a control 
group to demonstrate. Thus, methodological issues in the present study limit the interpretability of the quantitative results. With this in mind, Hypothesis 3.1 (no decline and possibly improvement in some scores) was generally supported, since as a group, the participants did not decline on any cognitive scores, improved on two cognitive measures, but also demonstrated a decrease on one self-report score (concern about dying).

Significant improvements were observed on a measure of visuospatial skills and a measure of delayed visual memory, with small-to-medium effect sizes. Additional analyses indicated that these changes in group scores were attributable to female participants' improvements, but not male older adults. When the analyses were run separately for men and women, men did not demonstrate changes in scores, but females improved significantly on visuospatial reasoning and delayed visual memory with large effect sizes (-1.84 and -1.35 , respectively). Although following Bonferroni correction, only one of these scores was significant (visuospatial skills), large effect sizes point to meaningful changes in females' scores from pre- to post-intervention.

One possible explanation for this finding is that the cognitive training tasks were more effective for visual processing skills, rather than verbal or auditory skills. However, this does not explain the fact that only females significantly improved on visual measures. A different explanation may be more plausible as it addresses gender differences. Because females scored significantly lower on visuospatial and delayed visual memory measures compared to males (and the population mean) at pre-test, and they were also the subgroup of participants who exhibited significant improvements in these cognitive skills, it can be suggested that participants with more pronounced 
weaknesses in specific functions are more likely to improve their functioning in these weaker areas and benefit most from the intervention.

The fact that individuals with lower baseline scores on visuospatial and delayed visual memory measures improved in these areas also suggests that identifying weaker cognitive functions in older adults and using cognitive training tasks to target these functions may be a more effective strategy than generalized or multi-domain cognitive training. This strategy was employed by Shatil (2013) who administered cognitive training tasks to the participants based on their weaknesses at baseline. However, other research findings suggested that multi-domain, generalized cognitive training is more efficacious compared to single-domain cognitive training (e.g., Cheng et al., 2013). Using individualized approach based on the participants' weaknesses would be difficult in dyadic cognitive training since it would require two manuals per dyad (unless both participants exhibit weaknesses in the same cognitive domains) and may be overly repetitive due to small variability of tasks, contributing to boredom and loss of motivation.

Individual evaluation of participants' cognitive scores revealed that while the majority of test scores remained stable from pre- to post-intervention assessment, the participants exhibited both increases and decreases in certain scores, with more increases than decreases observed. A change of one standard deviation or more was used for this exploratory analysis, which is an arbitrary benchmark. Interestingly, a number of participants improved in their visuospatial skills and language, but more participants had a decrease in scores on measures of attention/ multitasking and delayed memory compared to those who improved in these skills. Forty-three percent of participants 
improved on at least one measure of executive functioning, and the majority (71 percent) improved on at least one RBANS-U cognitive domain. Two illustrative case studies demonstrated that the participants' scores increased by at least one standard deviation on a number of cognitive tests (in some cases by two standard deviations or more), which points to the need to further explore this intervention in a larger sample.

It is important to keep in mind that in the absence of a control group, it cannot be determined whether the improvements were attributable to increased socialization, increased general engagement, maturation, practice effects, or other factors. The potential effects of increased social and general engagement have been addressed in studies by employing an active (or placebo) control group, which is a crucial component in cognitive training research. Maturation is unlikely in this case, since the natural course of cognition is gradual decline in older adults. As for the practice effects, different forms of RBANS-U were administered at pre- and post-test to address this issue, and the period between assessments was substantial ( 7 to 24 weeks). Also, if improvements were caused by practice effects, it is not clear why there was a lack of similar improvement on other components of the same measures and why there were some decreases in scores among participants. It is also difficult to determine whether decreases in cognitive scores occurred due to natural decline, situational variables, or other factors.

On a side note, one of the issues discussed by cognitive training researchers is the question of transfer, or whether the effects of training expand to untrained domains. Neartransfer refers to domains that are close to the targeted functions, while the far-transfer refers to domains that are distinctively different from the trained skills (Jaeggi, Buschkuehl, Shah, \& Jonides, 2013). The results of the study point to near-transfer since 
visual memory was not among the functions targeted by the intervention. Another question that arises in cognitive training research is whether cognitive training transfers onto IADLs. In the study, a measure of self-reported and spouse-reported (in spousal dyads) daily functioning was included, but was not related to any of the cognitive outcomes. Also, the participants did not have significant changes in scores from before to after the intervention on any of the self-report measures. An exception was increased concern about dying, which may stem from maturation/aging factors. Due to subjectivity of such measures and lack of follow-up assessment in the study, future studies need to incorporate objective measures of daily functioning that require participants perform simple tasks using common objects that simulate daily activities (e.g., "Timed ADL Tasks,” Owsley, Sloane, McGwin, \& Ball, K., 2002).

Lastly, Research Question 4, "is there a relationship between participant characteristics and their scores on the outcome measures?" was evaluated. Hypothesis 4.1 (lower education, older age, and lower health status related to lower pre-test cognition; no effect of gender) was rejected, since education was not linked with cognition, older age was related to higher language scores only, and females scored lower than males on some measures. However, lower scores on certain aspects of health did correspond with lower scores on several cognitive measures.

As noted earlier, maintaining cognitive vitality in older age and slowing down cognitive decline or delaying its onset are the main goals of cognitive training interventions (Ball et al., 2014). Thus, establishing efficacy of cognitive training requires an active control group and extensive follow-up assessments. Nevertheless, group and individual improvement in some cognitive functions is an important and promising 
finding in this feasibility study. It provides preliminary evidence that dyadic, selfadministered approach to cognitive training may help address cognitive decline in healthy older adults.

\section{Qualitative Findings}

Overall, the qualitative data demonstrated that self-administered, dyadic approach with coaching was feasible and was characterized by high participant acceptability. In their interviews, the participants reported that participating in cognitive training with a study partner contributed to their motivation and helped them to maintain their selfdiscipline and focus. It also provided them with an opportunity to socialize, spend time with each other, and learn more about one another.

As expected, spousal dyads met at home. Non-spousal dyads met at public locations that were convenient for them, including a senior center, coffee shop, and common area in an apartment building. The participants indicated that they appreciated flexibility of the intervention and the ability to have sessions at home or another location of their choice. They did not report difficulty coordinating schedules, although some older adults said that it took an effort to make the time for the sessions. Not having to travel was an important aspect of cognitive training, as a number of individuals stated that they would not have participated if this were not the case. This highlights the fact that onsite interventions may not be accessible to broader older adult population, and it is necessary for research and community programs to adopt to the needs of the older adults in order to ensure their access to cognitive training, enhance external validity of such programs, and increase their relevance to existing modes of service delivery. 
With regard to frequency and duration, some participants stated that 12 weeks was too long for the intervention. The two dyads that requested to finish earlier made that decision due to issues with the content of the intervention, as opposed to the overwhelming duration of the intervention. Some participants said that twice a week schedule worked for them, while others reported that once a week would have been more reasonable, and that is what they had been managing. On the other hand, two dyads wished that there were more tasks to work on and more sessions.

The content of the intervention and the nature of tasks was the main concern among the participants. The majority of participants indicated that many tasks were too easy and that they appreciated more challenging exercises. For some older adults, this contributed to boredom and loss of interest in the intervention, and two dyads requested to shorten its duration. A few participants questioned the value of the intervention and did not see the connection between some visual tasks and cognitive functioning. One participant suggested that a brief explanation of how specific tasks relate to daily cognition is included. Also, a number of participants stated that they wished there was more of an increase in difficulty in the exercises.

Since the tasks were adopted from the cognitive rehabilitation books developed for individuals with neurological damage, more difficult tasks were selected from these sources. Nevertheless, some of the exercises were too simple for the sample, and future studies need to incorporate more challenging tasks with a sharper increase in difficulty for healthy older adults. However, it is noteworthy that the sample was highly educated and cognitively engaged, and it is possible the same tasks would pose more of a challenge 
for older adults with lower baseline cognitive status, lower health status, lower education, or other factors.

Several participants were aware of their strengths and weaknesses, as they stated that they were better at visual or verbal tasks. Some participants reported that they felt that their performance on tasks was improving with time. A few participants noted that some tasks offered them a new perspective on the topic, allowed them to learn something new, or triggered further exploration of the subject. Others reported that they were becoming tired of the intervention because some tasks were overly tedious and repetitive.

Most older adults commented on enjoying verbal memory exercises that involved listening to a story and answering questions about it, which they also found challenging. Also, participants reported that they liked visual and verbal coding exercises, guessing what the word is based on clues, and language tasks. The task that the majority of respondents disliked and found boring, tedious, and repetitive was a task of visual attention, which required crossing out letters in a string of letters that also appeared in the keyword and counting them. Some older adults complained that the font was too small and narrow, which made it strenuous on their eyes. It would probably be acceptable to include this task in future studies, as long as the font is adequate, it is not repeated too many times, and it is explained that the task targets visual attention and scanning abilities.

A number of participants found a verbal deduction task that required listening to clues and filling out a table about several characters to be too difficult. Many participants skipped that exercise. However, this was one of the participants' favorite task, who appreciated the challenge and was disappointed that this exercise was removed from the second binder of tasks. Similarly, some older adults indicated that they did not like open- 
ended, discussion tasks, while others reported enjoying them, and some others found them silly.

The format of the manual and the fact that it was shared did not cause any issues among the participants. The individuals mentioned that on a few occasions there were errors and unclear instructions, which needs to be addressed in future studies. Also, it appeared that it was not clear to some of the participants how to use the log sheet, as they used two rows per session instead of one. The log sheets can be easily modified in the future. Additionally, it seems that for tasks that include a Work Sheet, it would be easier if this sheet is placed before the page with task instructions rather than after. Lastly, there needs to be a larger compartment in the manual to store the used Work Sheets. Based on the older adults' feedback, the 30-minute sand clock is a good visual tool to keep track of time during the sessions, which works best for dyads meeting at home. For dyads who meet outside the home or travel, the sand clock may not be practical as it can fall on the side and take a long time to reset. Electronic timer with a beeper would be preferable in cases where cognitive training sessions take place outside the home.

One of the concerns regarding the dyadic format was dyad interaction and a risk of negative feelings or conflicts. The vast majority of participants stated that being the trainer or being the student during cognitive training sessions did not create interpersonal problems. Few participants reported that initially, there was some nervousness about giving correct answers and also a feeling of competitiveness, but this was not the case in later sessions. Also, all participants stated that dyadic format did not affect their interaction or relationship in a negative way. It appeared that having a pre-existing positive relationship contributed to having a positive experience in dyadic training. For 
the unfamiliar dyad, having similar approach to training and similar interests helped the participants get along and work well together.

When asked whether they noticed any differences in cognition as a result of the intervention, a number of participants reported being more aware of the importance to pay attention in their daily lives. While a few participants stated that they felt their cognitive skills became stronger, most older adults said that they did not notice any improvements in cognitive skills or daily functioning. With regard to the overall experience, the participants indicated that they enjoyed taking part in the study since they appreciated an opportunity to do something new and something different, increase mental stimulation, contribute to research, and help the researcher's academic work. Some participants offered ideas with regard to applying the dyadic concept to other populations or developing a cognitive training board game in older adults.

It can be concluded regarding Research Question 2, "What are the participants' experiences with the intervention?" that for the most part, the participants responded positively to the novel approach. With some modifications in content, materials, and duration, the dyadic intervention can be utilized in larger studies and RCTs in order to evaluate its efficacy.

\section{Study Strengths and Limitations}

Strengths. The major strength of the feasibility study is that it evaluated a novel intervention for cognitive decline in healthy older adults. As older adults live longer and age-related cognitive changes increasingly affect their quality of life, it is important to investigate interventions that may contribute to cognitive health in this population. This may be particularly true for non-pharmacological interventions that are relatively low 
cost, flexible and accessible to a wide range of individuals, and are similar to cognitive training programs that have already been demonstrated to be effective in healthy older adults.

The novelty of the intervention is in its dyadic home-based format, with older adults taking turns to train one another. It appears that to date, only one dyadic intervention for cognitive decline has been tested in older population. Margrett and Willis (2006) employed home-based cognitive training among older couples; however their intervention was based on partner collaboration, rather than coaching and turn-taking. Collaboration may result in one partner taking the lead on tasks, while the other partner is less actively involved, and one partner may compensate for the cognitive weaknesses of the other. In the study, both partners had to work on tasks individually as they trained one another for 30 minutes each. Based on an extensive literature search, partner coaching and turn-taking have not been explored in cognitive training research.

Another strength of the study is that the flexible format of the intervention likely contributed to its external validity. The fact that the participants engaged in the intervention at home or another convenient location minimized issues related to scheduling, mobility, caretaking, transportation, and weather conditions, all of which may arise with onsite interventions. Home-based approach may be accessible to older adults who are frail, have chronic illnesses, physical disability, caregiving responsibilities, and transportation issues. It can potentially include older adults who are most likely to be in need of, and/or to benefit from cognitive training interventions, and yet are typically excluded from conventional cognitive training studies due to stringent inclusion criteria and high participant burden. 
Thus, the sample in the study may be more representative of the target population in terms of mobility and health since it does not exclude individuals unable to travel to a research site for various reasons. This conclusion is supported by the qualitative data, as older adults shared that the convenience of the intervention was one of the determining factors in their decision to participate, which likely reflects the attitudes of the broader older adult population. External validity was also enhanced by including participants over the age of 65 without the age limit. Some previous studies excluded older adults over the age of 75 (e.g., Mosolic et al., 2011), which results in a sample that is not representative of the general older adult population.

Paper-and-pencil format of the intervention contributed to its flexibility and relatively low cost, since no computers or internet connection were needed. Additionally, paper-and-pencil format does not raise issues related to computer stress and computer fatigue. In one study, computer stress was among the causes of attrition, and the attrition rate was very high (48 percent; Shatil, 2013). In another study on computerized cognitive training, the attrition rate was also high (35 percent, Corbett et al., 2015). These findings confirm that computerized approach is not suitable for all older adults, and paper-andpencil programs should also be developed and evaluated based on the needs of the older adult population.

The format and setting of the intervention may also provide a considerable level of flexibility on the part of agencies which may employ this cognitive training program in the future. Since the intervention does not require facilities or computers and is associated with a relatively low level of staff involvement, it may present a feasible option for agencies with limited budget and resources. For instance, a cognitive training "group" or 
"class" can be offered in senior centers along with fitness and recreational programs. Another strength of the intervention is that it promotes older adults' empowerment and self-determination. Older adults are provided tools to increase their cognitive engagement, but it is their choice when, where, and with whom cognitive training takes place. This approach can help older adults recognize that they have the power to make the changes in their lives that would promote their well-being, and also become more aware of the benefits of involving peers in this process. Dyadic approach demonstrates that older adults can use each other as a resource and as a support, which can foster meaningful relationships and contribute to their social engagement and quality of life.

Among the advantages of the study design is utilization of RBANS-U (Randolph, 2012) as the main outcome measure assessing cognition. This battery of cognitive tests was specifically designed to provide a brief, yet comprehensive assessment of various cognitive domains and allow for re-assessment within a short period of time, which is important when dealing with older adults. Two different forms of RBANS-U were administered to the participants at pre-and post-intervention to minimize practice effects, which may be an issue particularly when it comes to measures of verbal memory that include stories and word lists. Because the participants were exposed to an entirely different set of items the second time they were tested, it was expected that prior RBANS-U administration did not significantly affect their results.

Further, qualitative component of the study provided in-depth information about participants' experiences with the intervention. Allowing older adults to voice their opinions about the intervention is a vital part of developing a behavioral treatment, since 
ultimately, they are the consumers, and the success of the intervention depends on their acceptance of the program. Qualitative interviews shed light on what worked and what did not work for older adults in terms of format, setting, content, materials, facilitation, and frequency and duration of the intervention. This feedback is vital for making modifications to the cognitive training program for subsequent trials and informs future studies utilizing the dyadic intervention, as well as other studies on cognitive training in older adults.

Another strength of the study is that it is geared towards the social work audience, with a goal of raising the social workers' awareness about the importance of cognitive vitality, ways to address cognitive decline, and the relevance of the dyadic cognitive training in community settings. Since cognitive intervention research typically involves the fields of psychology, neuropsychology, psychiatry, nursing, and neurology, there is a need to increase the involvement of social workers in the area of cognition and aging. Social workers can play an important role in promoting cognitive vitality in older adults by advocating for cognitive training programming, doing community outreach, and facilitating cognition-based interventions both in research settings and in local agencies serving older adults. These implications are discussed in more detail in the Relevance to Social Work section.

Limitations. It may be argued that small sample size, lack of a control group, and lack of follow-up assessment undermine the design of the study. However, it is important to keep in mind that the primary goal of the study was to assess feasibility of a novel intervention and address any potential issues before efficacy studies can take place. Feasibility (Stage I) studies are typically characterized by small samples since the goal is 
to make appropriate modifications and specify the details of the intervention before moving on to larger, Stage II efficacy trials (Rounsaville \& Carroll, 2001). Nevertheless, the number of participants who completed all parts of the study $(N=14)$ was smaller than the minimum number of 20 indicated by the a-priori power analysis, which impacted the statistical power of the results and the ability to interpret the findings. Attrition rate of $22 \%$ was comparable to other cognitive training studies.

Even though quantitative component was secondary in this study, some limitations are worth noting. Insufficient diversity of the sample was a weakness, as the majority were educated Caucasian older adults. There were more females than males in the sample, and only two participants were Hispanic/Latino, while only one was AfricanAmerican. As a result, the sample was not representative of the general population. Demographic characteristics of the sample were largely due to the eligibility criterion of English language fluency. While there is a large Hispanic/Latino older adult population in South Florida who tend to have lower level of education, it appears that many of them are not fluent in English. Translating intervention materials and acquiring assessment instruments in Spanish was not feasible in the study. On the other hand, many of Caucasian older adults in this area are retirees from other states, and have higher education and income, which explains the unusually high educational level in the sample.

Practice effects are always a potential issue when cognitive measures are included in pre-test - post-test studies. Although the shortest period of time between the two assessment sessions was 7 weeks and two forms of RBANS-U were utilized, there was still a risk of practice effects on RBANS-U, and particularly on Stroop and TMT. The fact that some participants' performance remained stable or decreased from first assessment to 
the second suggests that practice effects did not influence all scores to the same degree. The issue of practice effects was also complicated by the fact that there was a wide range in time between baseline and post-intervention assessment among the participants. Future research should incorporate reliable change scores, which account for age-related cognitive changes and practice effects in participants.

Exposure bias could also affect the results of the study. First, the participants who withdrew from the study differed from those who did not on their attention scores and several self-report scores. It is possible that if these individuals completed the post-test assessment the sample scores would have been different. Second, the fact that there was a substantial variation in the number of sessions completed, number of weeks of intervention, and number of weeks between the two assessment sessions among the participants complicates interpretation of the results. The participants received different "dose" of the intervention over different time periods, which likely affected their response to treatment. The difference in dosage was partially due to the self-administered format of the intervention, which ensured its flexibility at the cost of adherence problems. Additionally, Thanksgiving and Christmas holidays coincided with the intervention for a number of dyads, which also contributed to adherence issues. Moreover, several dyads were able to complete all tasks within shorter number of sessions, which points to another limitation in the study- insufficient number of tasks for 24 sessions.

As previously discussed, an important limitation in the study was also the absence of an objective, performance-based measure of IADLs (in addition to a self-reported ADL scale), which is a common caveat in cognitive intervention studies (Papp et al., 2009). Performance-based measures typically require an extensive set of real-life testing items, 
such as a shelf and a telephone (e.g., Timed ADL, Owsley et al., 2002), which was not feasible in the study. It is expected that maintained or improved cognitive performance translates into functional outcomes (Ball et al., 2014), and future studies need to incorporate an objective measure of IADLs. In addition, it would have been beneficial to include measures of verbal and non-verbal reasoning in the study, since the intervention targeted this cognitive domain. Also, because of the peer-based approach, dyad relationship or interaction might have affected treatment outcomes, adherence, and other factors in the study. Incorporating a measure of relationship/ interaction dynamics in future studies on dyadic cognitive training will allow exploring the potential link between interpersonal factors, cognition, and efficacy of dyadic cognitive training.

Lastly, researcher bias must be mentioned among the limitations, since the researcher performed all assessments, data scoring and analysis, and was invested in the success of the cognitive training intervention, which might affect the results. This limitation can be addressed in future RCTs by utilizing an active control group and blinding assessors to the participants' experimental condition. All interviews were also coded by the researcher and the resulting codes were not cross-validated by other researchers. This might have resulted in limited codes or miscoded participant responses. On a side note, the intervention manual was not environmentally conscious, as it required printing of about 500 pages per dyad. In the future, this can be addressed by laminating the pages and using an erasable marker to mark answers on Work Sheets, which would allow re-using the manual.

\section{Significance of the Study}

The study is significant as it evaluates a unique cognitive training intervention, 
which has not been previously employed in the literature. To date, very few studies have utilized home-based cognitive training, and there is a lack of studies that include dyadic, peer-learning approach. The results of the study contribute to the existing literature on cognition-based interventions for cognitive decline in healthy adults, and may stimulate research focusing on dyadic cognitive training, as well as replication studies.

One of the main benefits of home-based dyadic cognitive training is that it is easily accessible and can accommodate broader older population. It is convenient and flexible, since it does not require travel or the use of a computer. Dyadic format can also increase socialization among older adults, which has been found to be beneficial in this population (Zunzunegui et al., 2003). This intervention is also relatively economical, as it does not require transportation services, scheduling, agency space, or computers, and involves limited supervision (Margrett \& Willis, 2006). In the light of these advantages, the finding that this approach is feasible and well-received by the participants is an important foundation for future research.

Combined with some promising statistical data in the study, qualitative findings confirm that dyadic approach to cognitive training is feasible, is characterized by high participant acceptability, and may help address cognitive decline and possibly improve some cognitive skills in cognitively healthy older adult population. The study contributes to the existing knowledge on cognitive vitality in older adults and ultimately will help promote independence and well-being among the growing older adult population and their families, also reducing the healthcare and economic burdens on the society.

\section{Relevance to Social Work}

Because it is associated with negative effects on individual, family, and societal 
levels, the problem of age-related cognitive decline is highly relevant to the social work profession. Social workers deal with older adults in a variety of settings, and their involvement in gerontology is likely to increase as the proportion of older adults continues to grow. Social workers are frequently involved in providing services to older adults with cognitive impairment and dementia and related functional deficits. However, there appears to be a lack of social workers involved in preventing and addressing agerelated cognitive changes in practice and in research. There is a need to increase social workers' presence in the cognition subfield of gerontology, currently dominated by neuropsychologists, neurologists, and other professionals.

The study illustrates that social workers can become more involved in the arena of cognition on individual, family, organizational, and policy levels. Specifically, social workers may lead cognition-based interventions in community and clinical settings. Since they are employed in senior centers, assisted living facilities, hospitals, and other agencies and institutions that provide services to older adults, they may be involved in cognitive training as facilitators, whether it be onsite or home-based cognitive programs, and train other professionals in administering cognitive interventions. The Social Work profession recognizes the importance of evidence-based practice (National Association of Social Workers [NASW], 2008), and the social workers need to be aware of the latest developments in the field, advocate for promising interventions and help implement them, facilitate such interventions, and adhere to the intervention protocols. They may also engage in appropriate program evaluation to assess the results of interventions and disseminate the results among gerontologists through peer-reviewed publications and presentations at professional conferences. 
In addition, social workers may take upon the role of educators with regard to cognitive health. Results of a national survey revealed that the general public is concerned about cognition, but many Americans believe that Alzheimer's disease is a part of normal aging (Connell, Roberts, \& McLaughlin, 2007). Social workers may educate older clients and caregivers, as well as their colleagues, other professionals, and direct care workers about normal vs. pathological cognitive decline and its risk and protective factors. Drawing on core social work values and priorities such as promoting selfdetermination, empowerment, and resilience (NASW, 2008), al workers should encourage older adults to consider their brain health and cognition as part of their overall health, and to view themselves as capable of achieving their health goals. In addition, social workers may facilitate interdisciplinary collaboration among nursing, medical, neurology, and neuropsychology professionals when it comes to older adults' cognition. Such collaboration may take place when dealing with individual patients, wherein social workers may help coordinate providers and ensure the patient and his or her providers are informed and are on the same page in term of test results (cognition, imaging, physical health, etc.) and treatment options. Interdisciplinary collaboration may also take place when developing and evaluating programs and services with an emphasis on cognitive health for older adults.

Even though at this time there is a lack of consensus on best practices with regard to cognitive training, some compelling evidence pointing to its effectiveness and longlasting benefits has emerged. Due to their administrative and provider positions and their vast linkages in the community, social workers may engage in efforts to make cognitive interventions available and accessible to older adults. They may advocate on the 
organizational and policy levels for cognitive training to be offered by senior centers and other agencies, and to be covered by the Older Americans Act. Thus, there are many ways in which social workers may be instrumental in promoting cognitive vitality among the growing aging population.

In addition to increased involvement of social workers in the areas of prevention and intervention for natural cognitive decline, there are other implications of cognitive training research for the Social Work profession. Prolonged cognitive vitality in older population may contribute to the numbers older adults over the age of 80 years who display minimal declines in cognitive function. This growing population is likely have a unique set of needs, and social workers may be required to fill this niche. For instance, while having intact cognitive functions, super agers may struggle with chronic health conditions and functional limitations. They may be actively involved in their healthcare and social workers may help these older adults evaluate and choose home-based and outpatient services and providers that are best suited to meet their individual needs. On the other end of the spectrum, super agers may be in good physical health and require recreation and social services that will interest and engage them and help them remain active. Again, social workers can help develop and implement such activities for older adults.

There are also economic and community-level factors related to improved cognitive vitality among older adults that may have implications for the Social Work profession. Advances in cognitive training research, in addition to the existing and new developments in prevention of cognitive impairment and chronic diseases are likely to lead to improved health, independence, and increased longevity of the growing older 
population in the US. However, these positive changes may be associated with such challenges as a shortage of health and community services, insufficient retirement and/or social security funds, and potentially increased homelessness among older adults. There may be a growing need for social workers to respond to these issues and provide services to older adults who lack resources essential for their well-being, as well as to address caregiver needs.

According to the NASW (2008) code of ethics, the social workers' mission is to "enhance human well-being and help meet basic human needs of all people" (para. 1). The study contributes to this mission since cognitive health is directly related to independence, quality of life, and well-being of older adults and their caregivers. Additionally, the code states that social workers aim "to enhance the capacity of people to address their own needs" (para. 2), and value family and other relationships. Dyadic cognitive training evaluated in the study provides the tools that older adults can use to enhance or maintain their own cognition and assist their loved ones, which aligns with social work goals and promotes self-determination and social support. Thus, the study conforms to the key principles of the Social Work profession and illuminates the various roles that the social workers can play in promoting cognitive health in older adults on the micro, mezzo, and macro-levels. 


\section{REFERENCES}

Administration on Aging, Department of Health and Human Services (2016). Aging statistics. Retrieved from http://www.aoa.acl.gov/aging_statistics/index.aspx

Administration on Aging, Department of Health and Human Services (2008). 2008 reports. Retrieved from http://www.aoa.gov/aoaroot/program_results/spr/2008/index.aspx

American Psychological Association (2011). Task force to update the guidelines for the evaluation of dementia and age-related cognitive decline. Guidelines for the evaluation of dementia and age-related cognitive decline. Retrieved from http://www.apa.org/practice/guidelines/dementia.aspx\#

Angevaren, M., Aufdemkampe, G., Verhaar, H. J. J., Aleman, A., \& Vanhees, L. (2008). Physical activity and enhanced fitness to improve cognitive impairment (review). Cochrane Database of Systematic Reviews, Issue 3. Art. No.: CD005381.

ATLAS.ti Scientific Software Development (2014). ATLAS.ti 7.5 Qualitative Data Analysis. Retrieved from http://www.atlasti.com/index.html

Ball, K., Edwards, J., Ross, L. A., \& McGwin, G. (2010). Cognitive training decreases motor vehicle collision involvement of older drivers. Journal of American Geriatrics Society, 58, 2107-2113.

Ball, K., Ross, L. A., Roth, D. L., \& Edwards, D. (2013). Speed of processing training in the ACTIVE study: Who benefits? Journal of Aging Health, 25(8), 65S-84S.

Barry, L. C., Murphy, T. E., \& Gill, T. M. (2011). Depressive symptoms and functional transitions over time in older persons. American Journal of Geriatric Psychiatry, 19(9), 783-791.

Baumgart, M., Snyder, H. M., Carrillo, M. C., Fazio, S., Kim, H., \& Johns, H. (2015). Summary of the evidence on modifiable risk factors for cognitive decline and dementia: A population-based perspective. Alzheimer's \& Dementia, 11(6), 718-726.

Boyle, P. A., Yu, L., Wilson, R. S., Gamble, K., Buchman, A. S., \& Bennett, D. A. (2012). Poor decision making is a consequence of cognitive decline among older persons without Alzheimer's disease or mild cognitive impairment. PLoS ONE, 7(8): e43647.

Blazer, D. G. (2009). Depression in later life: Review and commentary. Focus- The Journal of Lifelong Learning in Psychiatry, 7(1), 118-136.

Brehmer, Y., Rieckmann, A., Bellander, M., Westerberg, H., Fischer, H., \& Bäckman, L. (2011). Neural correlates of training-related working-memory gains in old age. NeuroImage, 58, 1110-1120. 
Buman, M. P., Giacobbi, P. R. Jr., Dzierzewski, J. M., Aiken Morgan, A., McCrae, C. S., Roberts, B. L., \& Marsiske, M. (2011). Peer volunteers improve long-term maintenance of physical activity with older adults: A randomized controlled trial. Journal of Physical Activity and Health, 8(Suppl. 2), S257-266.

Calero, D., \& Navarro, E. (2011). Differences in cognitive performance, level of dependency and quality of life (QoL), related to age and cognitive status in a sample of Spanish old adults under and over 80 years of age. Archives of Gerontology and Geriatrics, 53(3), 292-297.

Carlson, M. C., Erickson, K. I., Kramer, A. F., Voss, M. W., Bolea, N., Mielke, M., ... Fried, L. P. (2009). Neurocognitive plasticity in at-risk older adults: The Experience Corps program. Journal of Gerontology: Medical Sciences, 64(12), 1275-1282.

Castilla-Rilo, J., López-Arrieta, J., Bermejo-Pareja, F., Ruiz, M., Sánchez-Sánchez, F., Trincado, R. (2007). Instrumental activities of daily living in the screening of dementia in population studies: A systematic review and meta-analysis. International Journal of Geriatric Psychiatry, 22(9), 829-836.

Carlson, M. C., Xue, Q.-L., Zhou, J., \& Fried, L. P. (2009). Executive decline and dysfunction precedes declines in memory: The Women's Health and Aging Study II. Journal of Gerontology: Medical Sciences, 64A(1), 110-117.

Cavallini, E., Pagnin, A., \& Vecchi, T. (2003). Aging and everyday memory: The beneficial effect of memory training. Archives of Gerontology and Geriatrics, 37(3), 241257.

Centers for Disease Control and Prevention, National Center for Injury Prevention and Control (2014). Costs of falls among older adults. Retrieved from http://www.cdc.gov/homeandrecreationalsafety/falls/fallcost.html.

Chappell, N. L., \& Reid, R. C. (2002). Burden and well-being among caregivers: Examining the distinction. The Gerontologist, 42(6), 772-780.

Chao, L. L., \& Weiner, M. W. (2010). ASL perfusion MRI predicts cognitive decline and conversion from MCI to dementia. Alzheimer's Disease and Associated Disorders, 24(1), $19-27$.

Cheng, Y., Wu, W., Feng, W., Wang, J., Chen, Y., Shen, Y., Li, Q., . . Li, C. (2012). The effects of multi-domain versus single-domain cognitive training in non-demented older people: A randomized controlled trial. BMC Medicine, 10, 30.

Choi, S. H., Na, D. L., Lee, B. H., Kang, S. J., Ha, C. K., Han, S. H., \& Erzigkeit, H. (2003). Validation of the Korean version of the Bayer activities of daily living scale. Human Psychopharmacology: Clinical \& Experimental, 18, 469-475. 
Clark, F., Heller, A. F., Rafman, C., \& Walker, J. (1997). Peer learning: A popular model for seniors education. Educational Gerontology, 23(8), 751-762.

Cohen, J. (1969). Statistical power analysis for the behavioural sciences. New York: Academic Press.

Cohen, J. (1973). Eta-squared and partial eta-squared in fixed factor ANOVA designs. Educational and Psychological Measurement, 33, 107-112.

Collaborative Institutional Training Initiative (CITI) Program (2015). Online courses. Retrieved from citiprogram.org/index

Colcombe, S., \& Kramer, A. F. (2003). Fitness effects on the cognitive function of older adults: A meta-analytic study. Psychological Science, 14(2), 125-130.

Connell, C. M., Roberts J. S., \& McLaughlin, S. J. (2007). Public opinion about Alzheimer disease among blacks, Hispanics, and whites: Results from a national survey. Alzheimer's Disease and Associated Disorders, 21(3), 232-240.

Corbett, A., Owen, A., Hampshire, A., Grahn, J., Stenton, R., Dajani, S. ... Ballard, C. (2015). The effect of an online cognitive training package in healthy older adults: An online randomized controlled trial. Journal of the American Medical Directors Association, 16(11), 990-997.

de Winter, J. C. F. (2013). Using the Student's $t$-test with extremely small sample sizes. Practical Assessment, Research, and Evaluation, 18(10), 1-12.

Emery, V. O. B. (2011). Alzheimer disease: Are we intervening too late? Journal of Neural Transmission, 118, 1361-1378.

Faul, F., Erdfelder, E., Lang, A.-G., \& Buchner, A. (2007). G*Power 3: A flexible statistical power analysis program for the social, behavioral, and biomedical sciences. Behavior Research Methods, 39, 175-191.

Federal Trade Commission (2016). Lumosity to pay \$2 million to settle FTC deceptive advertising charges for its "Brain Training" program. Retrieved from https://www.ftc.gov/news-events/press-releases/2016/01/lumosity-pay-2-million-settleftc-deceptive-advertising-charges

Field, A. (2013). Discovering Statistics Using IBM SPSS Statistics (4th ed.). London: Sage.

Folquitto, J.C., Bustamante, S.E.Z., Barros, S.B., Azevedo, D., Lopes, M.A., Hototian ... \& Bottino, C.M.C. (2007). The Bayer - Activities of Daily Living Scale (B-ADL) in the differentiation between mild to moderate dementia and normal aging. Revista Brasileria Psiquiatria, 29(4), 350-353. 
Folstein, M. F., Folstein, S. E. \& McHugh, P. R. (1975). Mini-mental state: A practical method for grading the cognitive state of patients for the clinician. Journal of Psychiatric Research, 12, 189-198.

Fratiglioni, L., Wang, H. X., Ericsson, K., Maytan, M., \& Winblad, B. (2000). Influence of social network on occurrence of dementia: A community-based longitudinal study. Lancet, 355, 1315-1319.

Gates, N. J., Sachdev, P. S., Singh, M. A., \& Valenzuela, M. (2011). Cognitive and memory training in adults at risk of dementia: A systematic review. BMC Geriatrics, $11(55)$.

Glymour, M. (2011). Aging and social intervention: Life course perspectives. In Handbook of aging and the social sciences. Retrieved from

http://ezproxy.fiu.edu/login?url=http://search.credoreference.com.ezproxy.fiu.edu/content /entry/estaging/aging_and_social_intervention_life_course_perspectives/0

Golden, C.J. \& Freshwater, S.M. (2002). The Stroop Color and Word Test: A Manual for Clinical and Experimental Uses. Wood Dale, IL: Stoelting Co.

Gontkovsky, S. T., Beatty, W. W., \& Mold, J. W. (2004). Repeatable Battery for the Assessment of Neuropsychological Status in a normal, geriatric sample. Clinical Gerontologist, 27(3), 79-86.

Gould, O., Kurzman, D., \& Dixon, R. A. (1994). Communication during prose recall conversations by young and old dyads. Discourse Processes, 17, 149-165.

Gross, A. L., \& Rebok, G. W. (2011). Memory training and strategy use in older adults: Results from the ACTIVE study. Psychology and Aging, 26(3), 503-517.

Hamami, A., Serbun, S. J., \& Gutchess, A. H. (2011). Self-referencing enhances memory specificity with age. Psychology and Aging, 26(3), 636-646.

Harrison, T. M., Weintraub, S., Mesulam, M.-M., \& Rogalski, E. (2012). Superior memory and higher cortical volumes in unusually successful cognitive aging. Journal of the International Neuropsychological Society, 18(6), 1081-1085.

Health and the life course. (2001). In World of Sociology, Gale. Retrieved from http://ezproxy.fiu.edu/login?url=http://search.credoreference.com.ezproxy.fiu.edu/content /entry/worldsocs/health_and_the_life_course/0

Hedden, T., \& Gabrieli, J. D. E. (2004). Insights into the ageing mind: A view from cognitive neuroscience. Neuroscience, 5, 87-97.

Henkel, L. A., \& Rajaram, S. (2011). Collaborative remembering in older adults: Ageinvariant outcomes in the context of episodic recall deficits. Psychology \& Aging, 26(3), 532-545. 
Herman, T., Mirelman, A., Giladi, N., Schweiger, A., \& Hausdorff, J. M. (2010). Executive control deficits as a prodrome to falls in healthy older adults: A prospective study linking thinking, walking, and falling. Journal of Gerontology, Medical Sciences. 65A(10), 1086-1092.

Hindmarch, I., Lehfeld, H., de Jongh, P., Erzigkeit, H. (1998). The Bayer Activities of Daily Living Scale (B-ADL). Dementia and Geriatric Cognitive Disorders, 9(suppl 2), 20-26.

Holtzman, R. E., Rebok, G. W., Saczynski, J. S., Kouzis, A. C., Doyle, K. W., \& Eaton, W. W. (2004). Social network characteristics and cognition in middle-aged and older adults. Journal of Gerontology B: Psychological Sciences \& Social Sciences, 59(6), 278284.

IBM Corporation (2012). IBM SPSS (21 $1^{\text {st }}$ ed.). Author.

Infurna, F. J., Gerstorf, D., Ryan, L. H., \& Smith, J. (2011). Dynamic links between memory and functional limitations in old age: Longitudinal evidence for age-based structural dynamics from the AHEAD study. Psychology and Aging, 26(3), 546-558.

Insurance Institute for Highway Safety (2014). Older drivers. Retrieved from http://www.iihs.org/iihs/topics/t/older-drivers/qanda.

Jaeggi, S. M., Buschkuehl, M., Shah, P., \& Jonides, J. (2013). The role of individual differences in cognitive training and transfer. Memory and Cognition, 42(3), 464-480.

Jones, T. G., Rapport, L. J., Hanks, R. A., Lichtenberg, P. A., \& Telmet, K. (2003). Cognitive and psychological well-being in urban older adults. The Clinical Neuropsychologist, 17(1), 3-18.

Jopp, D. S., Park, M.-K. S., Lehrfeld, J., \& Paggi, M. E. (2016). Physical, cognitive, social and mental health in near-centenarians and centenarians living in New York City: findings from the Fordham Centenarian Study. BMC Geriatrics, 16(1).

DOI: $10.1186 / \mathrm{s} 12877-015-0167-0$

Kanaan, S. F., McDowd, J. M., Colgrove, Y., Burns, J. M., Gajewski, B., \& Pohl, P. S. (2014). Feasibility and efficacy of intensive cognitive training in early-stage Alzheimer's disease. American Journal of Alzheimer's Disease and Other Dementias, 29(2), 150-158.

Katon, W. J., Lin, E., Russo, J., \& Unützer, J. (2003). Increased medical costs of a population-based sample of depressed elderly patients. Archives of General Psychiatry, 60, 897-903.

Kohler, S., van Boxtel, M. P. J., van Os, J., Thomas, A. J., O’Brien, J. T., Jolles, J., . . Allardyce, J. (2010). Depressive symptoms and cognitive decline in community-dwelling older adults. Journal of the American Geriatric Society, 58(5), 874-879. 
Kowalski, K., Love, J., Tuokko, H., MacDonald, S., Hultsch, \& Strauss, E. (2011). The influence of cognitive impairment with no dementia on driving restriction and cessation in older adults. Accident Analysis and Prevention, 49, 308-315.

Kramer, A. F., \& Willis, S. L. (2002). Enhancing the cognitive vitality of older adults. American Psychological Society, 11(5), 173-177.

Kroenke, K., \& Spitzer, R. L. (2002). The PHQ-9: A new depression diagnostic and severity measure. Psychiatric Annals, 32(9), 1-7.

Kurz, A. F., Leucht, S., \& Lautenschlager, N. T. (2011). The clinical significance of cognition-focused interventions for cognitively impaired older adults: A systematic review of randomized controlled trials. International Psychogeriatrics, 23, 1364-1375.

Laditka, S. B., Corwin, S. J., Laditka, J. N., Liu, R., Tseng, W., Wu, B., ... Ivey, S. L. (2009). Attitudes about aging well among a diverse group of older Americans:

Implications for promoting cognitive health. The Gerontologist, 49(S1), S30-S39.

Lampit, A., Hallock, H., \& Valenzuela, M. (2014). Computerized cognitive training in cognitively healthy older adults: A systematic review and meta-analysis of effect modifiers. PLoS Med, 11:e1001756.

Lampit, A., Valenzuela, M., \& Gates, N. J. (2015). Computerized cognitive training is beneficial for older adults. Journal of the American Geriatrics Society, 63(12), 26102615.

Lövdén, M., Schaefer, S., Noack, H., Bodammer, N. C., Kühn, S., Hans-Jochen Heinze, H.-J., ... Lindenberger, U. (2012). Spatial navigation training protects the hippocampus against age-related changes during early and late adulthood. Neurology of Aging, 33, 620.e9-620.e22.

Malia, K.B., Bewick, K.C., Raymond, M.J., \& Bennett, T.L. (2002). Brainwave-R series. Cognitive strategies and techniques for brain injury rehabilitation. Austin, TX: Pro-Ed, Inc.

Margrett, J. A., \& Willis, S. L. (2006). In-Home cognitive training with older married couples: Individual versus collaborative learning. Neuropsychology, Development, \& Cognition, 13(2), 173-195.

McKnight, A. J., \& McKnight, A. S. (1999). Multivariate analysis of age-related driver ability and performance deficits. Accident Analysis and Prevention, 31, 445-454.

MetLife Mature Market Institute (2011). The MetLife study of elder financial abuse: Crimes of occasion, desperation, and predation against America's elders. Retrieved from www.giaging.org/documents/mmi-elder-financial-abuse.pdf 
Mitrushina, M., \& Satz, P. (1991). Reliability and validity of the mini-mental state exam in neurologically intact elderly. Journal of Clinical Psychology, 47, 537-543.

Mody, L., Miller, D. K., McGloin, J. M., Div, M., Freeman, M., Marcantonio, E. R., . . . Studenski, S. (2008). Recruitment and retention of older adults in aging research. JAGS, $56,2340-2348$.

Montejo, P., Montenegro, M., Fernandez, M. A., \& Maestu, F. (2012). Memory complaints in the elderly: Quality of life and daily living activities. A population based study. Archives of Gerontology and Geriatrics, 54, 298-304.

Morris, P. E., \& Fritz, C. O. (2013). Effect sizes in memory research. Memory, 21(7), $832-842$.

Mozolic, J. L., Long, A. B., Morgan, A. R., Rawley-Payne, M., \& Laurienti, P. J. (2011). A cogntiive training intervention improves modality-specific attention in a randomized controlled trial of healthy older adults. Neurobiology of Aging, 32, 655-668.

Muir, S. W., Gopaul, K., \& Montero Odasso, M. M. (2012). The role of cognitive impairment in fall risk among older adults: A systematic review and meta-analysis. Age and Aging, 41, 299-308.

National Association of Social Workers (2008). Code of Ethics of the National Association of Social Workers. Retrieved from https://www.socialworkers.org/pubs/code/code.asp.

National Institutes of Health (1998). Rehabilitation of persons with traumatic brain injury. NIH consensus statement. Retrieved from http://www.nlm.nih.gov/archive/20040830/pubs/cbm/tbi.html.

National Institute on Aging, US National Institutes of Health (2009). 2009 Progress report on Alzheimer's Disease: Translating new knowledge. Retrieved from http:/www.nia.nih.gov/Alzheimers/Publications/ADProgress2009/

Newson, R. S., \& Kemps, E. B. (2005). General lifestyle activities as a predictor of cognition and cognitive change in older adults : A cross-sectional and longitudinal examination. Journal of Gerontology B: Psychological Sciences \& Social Sciences, 60(3), 113-120.

Norden, D. M., \& Godbout, J. P. (2013). Microglia of the aged brain: Primed to be activated and resistent to regulation. Neuropathology \& Applied Neurobiology, 39(1), 1934.

Owsley, C., Sloane, M., McGwin, G. Jr., \& Ball, K. (2002). Timed Instrumental Activities of Daily Living Tasks : Relationship to cognitive function and everyday performance assessments in older adults. Gerontology, 48, 254-265. 
Papp, K. V., Walsh, S. J., \& Snyder, P. J. (2009). Immediate and delayed effects of cognitive interventions in healthy elderly : A review of current literature and future directions. Alzheimer's \& Dementia, 5, 50-60.

Park, D. C., \& Bischof, G. N. (2013). The aging mind : Neuroplasticity in response to cognitive training. Dialogues in Clinical Neuroscience, 15(1), 109-119.

Park, H. L., O'Connell, J. E., \& Thomson, R. G. (2003). A systematic review of cognitive decline in the general elderly population. International Journal of Geriatric Psychiatry, $18,1121-1134$.

Parker, R. I. (2006). Increased reliability for single-case research results. Is the bootstrap the answer? Behavior Therapy, 37(4), 326-338.

Pérès, K., Helmer, C., Amieva, H., Orgogozo, J. -M., Rouch, I., Dartigues, J.- F., \& Barberger-Gateau, P. (2008). Natural history of decline in instrumental activities of daily living performance over the 10 years preceding the clinical diagnosis of dementia: A prospective population-based study. Journal of the American Geriatrics Society, 56(1), $37-44$.

Perzynski, A. (2006). Disengagement theory. In The encyclopedia of aging. Retrieved from http://ezproxy.fiu.edu/login?url=http://search.credoreference.com.ezproxy.fiu.edu/content /entry/spencage/disengagement_theory/0

Plassman, B. L., Williams, J. W., Burke, J. R., Holsinger, T., \& Benjamin, S. (2010). Systematic review: Factors associated with risk for and possible prevention of cognitive decline in later life. Annals of Internal Medicine, 153, 182-189.

Pusswald, G., Tropper, E., Kryspin-Exner, I., Moser, D., Klug, S., Auff, E. ... Lehrner, J. (2015). Health-related quality of life in patients with subjective cognitive decline and mild cognitive impairment and its relation to activities of daily living. Journal of Alzheimer's Disease, 47, 479-486.

Randolph, C., Tierney, M. C., Mohr, E., \& Chase, T. N. (1998). The Repeatable Battery for the Assessment of Neuropsychological Status (RBANS): Preliminary clinical validity. Journal of Clinical and Experiomental Neuropsychology, 20(3), 310-319.

Randolph, C. (2012). The Repeatable Battery for the Assessment of Neuropsychological Status- Update. San Antonio, TX: Pearson.

Raz, N., \& Lindenberger, U. (2011). Only time will tell: Cross-sectional studies offer no solution to the age-brain-cognition triangle: Comment on Salthouse (2011). Psychological Bulletin, 137(5), 790-795.

Rebok, G. W., Ball, K., Guey, L. T., Jones, R. N., Kim, H.-Y., King, J. W., . . Willis, S. L. (2014). Ten-year effects of the Advanced Cognitive Training for Independent and Vital 
Elderly cognitive training trial on cognition and everyday functioning in older adults. Journal of the American Geriatrics Society, 62(1), 16-24.

Rebok, G. W., Parisi, J. M., Gross, A. L., \& Spira, A. P. (2010). Assessment of cognitive training. In P. A. Lichtenberg (ed.), Handbook of assessment in clinical gerontology $\left(2^{\text {nd }}\right.$ ed.). (pp. 216-228). Amsterdam: Academic.

Reitan, R. M., \& Wolfson, D. (1993). The Halstead-Reitan neuropsychological test battery. Theory and clinical interpretation ( $2^{\text {nd }}$ ed.). Tucson, AZ: Neuropsychology Press.

Reuter-Lorenz, P. A., \& Park, D. C. (2010). Human neuroscience and the aging mind: A new look at old problems. Journal of Gerontology B: Psychological Sciences \& Social Sciences, 65(4), 405-415.

Rohwedder, S., \& Willis, R. (2010). Mental retirement. Journal of Economic Perspectives, 24(1), 119-138.

Rose, N. S., Rendell, P. G., Hering, A., Kliegel, M., Bidelman, G. M., \& Craik, F. I. M. (2015). Cognitive and neural plasticity in older adults' prospective memory following training with the Virtual Week computer game. Frontiers in Human Neuroscience, 9, 592.

Ross, L. A., Edwards, J. D., O’Connor, M. L., Ball, K. K., Wadley, V. G., \& Vance, D. E. (2015). The transfer of cognitive speed of processing training to older adults' driving mobility across 5 years. Journal of Gerontology B: Psychological Sciences \& Social Sciences, 71(1), 87-97.

Rounsaville, B. J., \& Carroll, K. M. (2001). A stage model of behavioral therapies research: Getting started and moving on from Stage I. Clinical Psychology: Science and Practice, 8, 133-142.

Royall, D. R., Lauterbach, M. D., Kaufer, D., Malloy, O., Coburn, K. L., \& Black, K. J. (2007). The cognitive correlates of functional status: A review from the committee on research of the American Neuropsychiatric Association. Journal of Neuropsychiatry and Clinical Neuroscience, 19(3), 249-265.

Sachdev, P. S., Levitan, C., Crawford, J., Sidhu, M., Slavin, M., Richmond, R. ... Mather, K. A. (2013). The Sydney Centenarian Study: methodology and profile of centenarians and near-centenarians. International Psychogeriatrics, 25(6), 993-1005.

Salthouse, T. A. (1999). Theories of cognition. In V. L. Bengtson \& K. W. Schaie (Eds.). Handbook of theories of aging (196-209). New York, NY : Springer Publishing Company, Inc.

Schaie, K. W., Willis, S. L., \& Caskie, G. I. (2004). The Seattle longitudinal study: Relationship between personality and cognition. Neuropsychology, Development, and Cognition. Section B, Aging, Neuropsychology, and Cognition, 11, 304-324. 
Seeman, T. E., Lusignolo, T. M., Albert, M., \& Berkman, L. (2001). Social relationships, social support, and patterns of cognitive aging in healthy, high-functioning older adults: MacArthur Studies of Successful Aging. Health Psychology, 20(4), 243-255.

Shatil, E. (2013). Does combined cognitive training and physical activity training enhance cognitive abilities more than either alone? A four-condition randomized controlled trial among healthy older adults. Frontiers in Aging Neuroscience, 5. doi: 10.3389/fnagi.2013.00008.

Sibley, A., MacKnight, C., Rockwood, K., Fisk, J., Gauthier, S., Guzman, D. A., \& Hogan, D. B. (2002). The effect of the living situation on the severity of dementia at diagnosis. Dementia and Geriatric Cognitive Disorders, 13, 40-45.

Singh-Manoux, A., Kivimaki, M., Glymour, M. M., Elbaz, A., Berr, C., Ebmeier, K. P. . . . Dugravot, A. (2012). Timing of onset of cognitive decline: results from Whitehall II prospective study. $B M J, 344: \mathrm{d} 7622$.

Slagter, H. A, Davidson, R. J., \& Lutz, A. (2011). Mental training as a tool in the neuroscientific study of brain and cognitive plasticity. Frontiers in Human Neuroscience, $5,1-12$.

Slosson, R. L., \& Nicholson, C. L. (2002). Slosson Oral Reading Test, Revised, $3^{\text {rd }}$ Edition. East Aurora, NY: Slosson Educational Publications.

Smith, G. E., Housen, P., Yaffe, K., Ruff, R. Kennison, R. F., Mahncke, H. W., \& Zelinski, E. M. (2009). A cognitive training program based on principles of brain plasticity: Results from the Improvement in Memory with Plasticity-based Adaptive Cognitive Training (IMPACT) Study. Journal of American Geriatrics Society, 57, 594603.

Smith-Ray, R. L., Hughes, S. L., Prohaska, T. R., Little, D. M., Jurivich, D. A., \& Hedeker, D. (2015). Journal of Gerontology B: Psychological Sciences \& Social sciences, 70(3), 357-366.

Solomon, P. (2004). Peer support/ peer provided services: Underlying processes, benefits, and critical ingredients. Psychiatric Rehabilitation Journal, 24(4), 392-401.

Spreen, O., \& Strauss, E. (1998). A compendium of neuropsychological tests:

Administration, norms, and commentary ( $2^{\text {nd }}$ Ed.). New York, NY: Oxfor University Press.

Stanford Center on Longevity (2014). A consensus on the Brain Training industry from the scientific community. Retrieved from http://longevity3.stanford.edu/blog/2014/10/15/the-consensus-on-the-brain-trainingindustry-from-the-scientific-community-2/ 
Tabbarah, M., Crimmins, E. M., \& Seeman, T. E. (2002). The relationship between cognitive and pjhysical performance : MacArthur Studies of Successful Aging. Journal of Gerontology, Medical Sciences, 57A(4), M-228-M235.

Tomlin, K. J. (2002). Workbook of Activities for Language and Cognition-2: Cognitive Rehab. East Moline, IL: LinguiSystems.

Taussig I. M., \& Pontón, M. (1996). Issues in neuropsychological assessment for Hispanic older adults: Cultural and Linguistic Factors. In G. Yeo \& D. GallagherThompson (Eds.), Ethnicity and the dementias.(pp. 47-58). Washington, DC: Taylor \& Francis.

Tranter, L. J., \& Koutstaal, W. (2008). Age and flexible thinking: An experimental demonstration of the beneficial effects of increased cognitively stimulating activity on fluid intelligence in healthy older adults. Aging, Neuropsychology, and Cognition, 15, 184-207.

Van Gelder, B. M., Tijhuis, M., Kalmijn, S., Giampaoli, S., Nissinen, A., \& Kromhout, D. (2006). Marital status and living situation during a 5-year period are associated with a subsequent 10-year cognitive decline in older men: The FINE Study. Journal of Gerontology B: Psychological Sciences \& Social Sciences, 61(4), 213-219.

Wakefield, D. B., Moscufo,N., Guttmann, C. R., Kuchel, G. A., Kaplan, R. F., Pearlson, G., \& Wolfson, L. (2010). White matter hyperintensities predict functional decline in voiding, mobility, and cognition in older adults. JAGS, 58, 275-281.

Ware, J. E. Jr, \& Sherbourne, C. D. (1992). The MOS 36-Item Short-Form Health Survey (SF-36). I. Conceptual framework and item selection. Medical Care, 30(6), 473-483.

West, R. L. (1996). An application of prefrontal cortex function theory to cognitive aging. Psychological Bulletin, 120(2), 272-292.

Wilk, C. M., Gold, J. M., Bartko, J. J., Dickerson, F., Fenton, W. S., Knable, M., . . . Buchanan, R. W. (2002). Test-retest stability of the Repeatable Battery for the Assessment of Neuropsychological Status in schizophrenia. American Journal of Psychiatry, 159, 838-844.

World Health Organization (WHO). 2006. WHOQOL-OLD: Manual. Retrieved from http://library.cphs.chula.ac.th/Ebooks/WHOQOL-OLD\%20Final\%20Manual.pdf

Williams, K. N., \& Kemper, S. (2010). Interventions to reduce cognitive decline in aging. Journal of Psychosocial Nursing, 48(5), 42-51.

Willis, S. L., Tennstedt, S. L., Marsiske, M., Ball, K., Elias, J., Koepke, K. M., Morris, J. N., et al. (2006). Long-term effects of cognitive training on everyday functional outcomes in older adults. Journal of American Medical Association, 296(23), 2805-2814. 
Yamamoto-Mitani, N., Matsuoka, K., \& Fujii, M. (2007). Home-based rehabilitation program for older adults with cognitive impairment: Preliminary results.

Psychogeriatrics, 7, 14-20.

Yang, Y., \& George,L. K. (2005). Functional disability, disability transitions, and depressive symptoms in later life. Journal of Aging and Health, 17, 263-292.

Zelinski, E. M., Dalton, S. E., \& Hindin, S. (2011). Cognitive changes in healthy older adults. Generations- Journal of the American Society on Aging, 35(2), 13-20.

Zunzunegui, M.-V., Alvarado, B. E., Del Ser, T., \& Otero, A. (2003). Social networks, social integration, and social engagement determine cognitive decline in communitydwelling Spanish older adults. Journal of Gerontology B: Psychological Sciences \& Social Sciences, 58(2), S93-S100. 


\section{APPENDICES}

Appendix 1

\section{Permissions to Use Measures}

\section{Slosson Oral Reading Test, Revised}

\section{SLOSSON EDUCATIONAL}

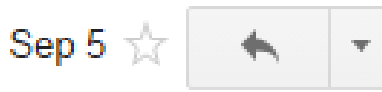

to me -

Hello Natalia

Thank you for your interest in our company and in the SORT-R3.

We will offer you a $20 \%$ discount off the SORT-R $3-1$ kit. Promo Code READ2o.

Permission is granted for use of the SORT-R 3 , we would like to have a copy of your results.

You can go to our website and sign up for field testing of the SORT-4.

Nothing may be reproduced. All materials are copyrighted.

Please order online with a credit card. www.slosson.com

SLOSSON EDUCATIONAL PUBLICATIONS INC

PO BOX 280

EAST AURORA NY 14052 


\section{Stroop Color and Word Test}

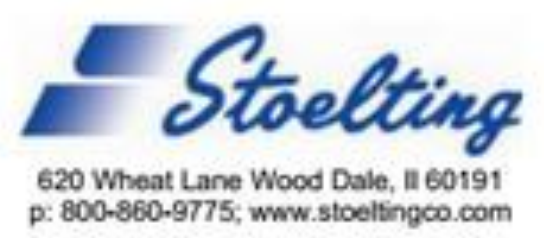

November $18^{\text {th }}, 2013$

Natalia Shtompel, M.A., MSW

Robert Stempel College of Public Health and Social Work

Florida International University

11200 SW 8th Street, GL 475B

Miami, FL 33199

Phone: 718-219-2884

Email: nshto001@fiu.edu

Dear Natalia,

Stoelting grants you permission to use Golden's Stroop Color and Word Test for your dissertation study "Home-Based Dyadic Cognitive Training for Healthy Older Couples: A Randomized Controlled Trial."

This permission is for research purposes only.

Should you need any other support, please do not hesitate to contact me.

Sincerely,

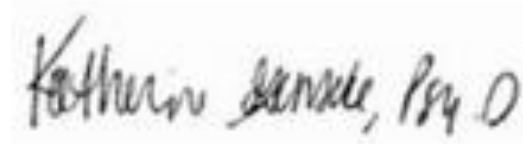

Katherine Genseke, Psy.D.

Product Manager for Psychological and Special Education Materials 


\section{Trail Making Test (TMT)}

$\therefore$ info

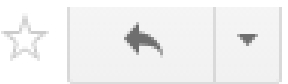

to me $\nabla$

As publisher of the Trail Making Test, we are giving permission to Natalia Shtompel to use the test in her dissertation study, entitled "Home-Based Dyadic Cognitive Training for Healthy Older Adults: A Pilot Trial." This permission is given with the understanding that she will honor the test copyright and will not make unauthorized copies of the test. It is understood that she will administer the test within the guidelines of the APA Standards for Educational and Psychological Tests (2014) and the APA Ethical Code of Conduct, including the requirement that she administer the test under qualified supervision.

The Neuropsychology Center, PC 
Repeatable Battery for the Assessment of Neuropsychological Status (RBANS-U)

\section{PEARSON}

ALWAYS LEARNING

CLINICAL ASSESSMENT

19500 Buhverde Rd

San Antonlo, Texas 73259

T: $800-627-7271$

F: $800-232-1223$

Natalia Sbtomegl,

The "Home Based Dyadic Cognifive Training for Healthy Older Adults: A Pilot Trial" research study has been approved to receive a $50 \%$ discount for the following assessments: RBANS.

This discount will be valid until March 31,2015 or the $\$ 5000$ (full retail value) cap is reached. Available amount for this study is $\$ 4234$ ( $\$ 766$ has been subtracted from the amount of your first order). Regretfully, no phone or web orders will be honored this discount. All quote and order requests must be faxed or emailed.

If you require additional materials for this study, please reference the following information on your next order: The name of the study. Agreement Code "RES", 50\% discount and Accourt \#1776009.

NOTE: Please be advised materials ordered for research cannot be returned. See paragraph 10 in the RAP Terms and Condi-

tions: http://psychcorp.pearsonassessments.com/pai/ca/support/rap/ResearchAssistanceProgram. htm.

If you have any questions regarding the order, please contact our Inbound Sales and Customer Support at 1-800-627-7271 or emailclinicalcustomersupport@pearson.com for assistance.

The order has been sent to our Order Management group for processing.

Sincerely,

Research Assistance Program (RAP)

Inbound Sales \& Customer Support

Clinical Assessment

Pearson

19500 Bulverde Road

San Antonio, TX 78259

$\mathrm{T}:(800) 627-7271$

$F:(800) 232-1223$

E: ResearchAssistQPearson.com 


\section{World Health Organization Quality of Life (WHOQOL-OLD)}

․ Donald Bushnell<bushnell@hrainc.net>

to me, Mona $\nabla$

Greetings Natalia,

Yes, you are permitted to use the WHOQOL-OLD module in your research. Please see attached a brief User Agreement, the manual (put out by the European study group) and the latest US English version (should you need it). I am assuming you have a copy of the paper.

Best of luck to you,

Don

Donald Bushnell | Associate Director

Health Research Associates, Inc.

Tel: +1 (425) 775-6565 Ext. 206 


\section{Bayer Activities of Daily Living (B-ADL)}

Lehfeld, Hartmut Dr. <Hartmut.Lehfeld@klinikum-nuernberg.de>

to me :

Dear Mrs. Shtompel,

thank you very much for your interest in the B-ADL scale. Please find attached for restricted use in your dissertation a copy of the scale. Scoring is easy:

The B-ADL total score is computed by summing the individual item scores excluding those completed "not applicable" or "unknown". The resut is divided by the number of items rated between 1 and 10. Thus, in the calculation of the total score, tems regarded as irrelevant (category "not appicable"), items for which a rating could not be made (category "unknown") and items not completed are replaced by the arithmetic mean of items for which existing difficulties were rated.

In return, I would be very interested to leam about the results of your work when you have finished the study.

Furthermore, a B-ADL manual has been published (unfortunately in German) which contains - amongst others - a survey on published literature on the scale:

htop:l/shop.pearsonassessment de/index,pho/cat/c154_B-ADL,html

The following table is taken from the manual and gives mean B-ADL scores for patients in GDS stages 1 to 5 (Reisberg Global Deteriration Scale)

\begin{tabular}{|l|c|c|c|c|c|c|c|}
\hline & & & \multicolumn{2}{|c|}{$95 \%$ Konfidenzintervall } & & & \\
\hline & mean & sd & $\begin{array}{c}\text { Unter- } \\
\text { grenze }\end{array}$ & $\begin{array}{c}\text { Ober- } \\
\text { grenze }\end{array}$ & Medlan & $\begin{array}{c}\text { Interquartl- } \\
\text { bereich }\end{array}$ & $n$ \\
\hline GDS 1 & 1,28 &, 46 & 1,18 & 1,38 & 1,12 & 0,40 & 78 \\
\hline GDS 2 & 2,36 & 1,20 & 2,12 & 2,60 & 2,04 & 1,39 & 97 \\
\hline GDS 3 & 3,58 & 1,87 & 3,30 & 3,86 & 3,13 & 2,76 & 174 \\
\hline GDS 4 & 5,01 & 2,00 & 4,73 & 5,28 & 4,91 & 3,21 & 207 \\
\hline GDS 5 & 7,09 & 1,97 & 6,77 & 7,40 & 7,39 & 3,07 & 153 \\
\hline gesamt & 4,33 & 2,56 & 4,15 & 4,52 & 4,00 & 4,41 & 709 \\
\hline
\end{tabular}

Please do not hesitate to contact me if you have any questions.

Best regards from Nürnberg, Germany

Dr. Hartmut Lehfeld

Leiter der Gedächtnissprechstunde 
Appendix 2

SORT-3

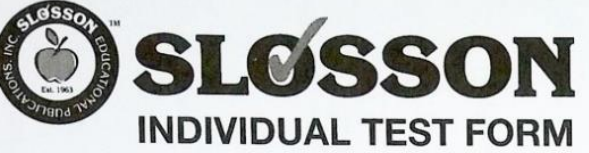

NAME
S0RTER

SLOSSON ORAL READING TEST

Richard L. Slosson

Revised by: Charles L. Nicholson

AGE DATE

Instrument excluded for copyright reasons. 


\section{Appendix 3}

\section{MMSE}

\section{The Mini-Mental State Exam}

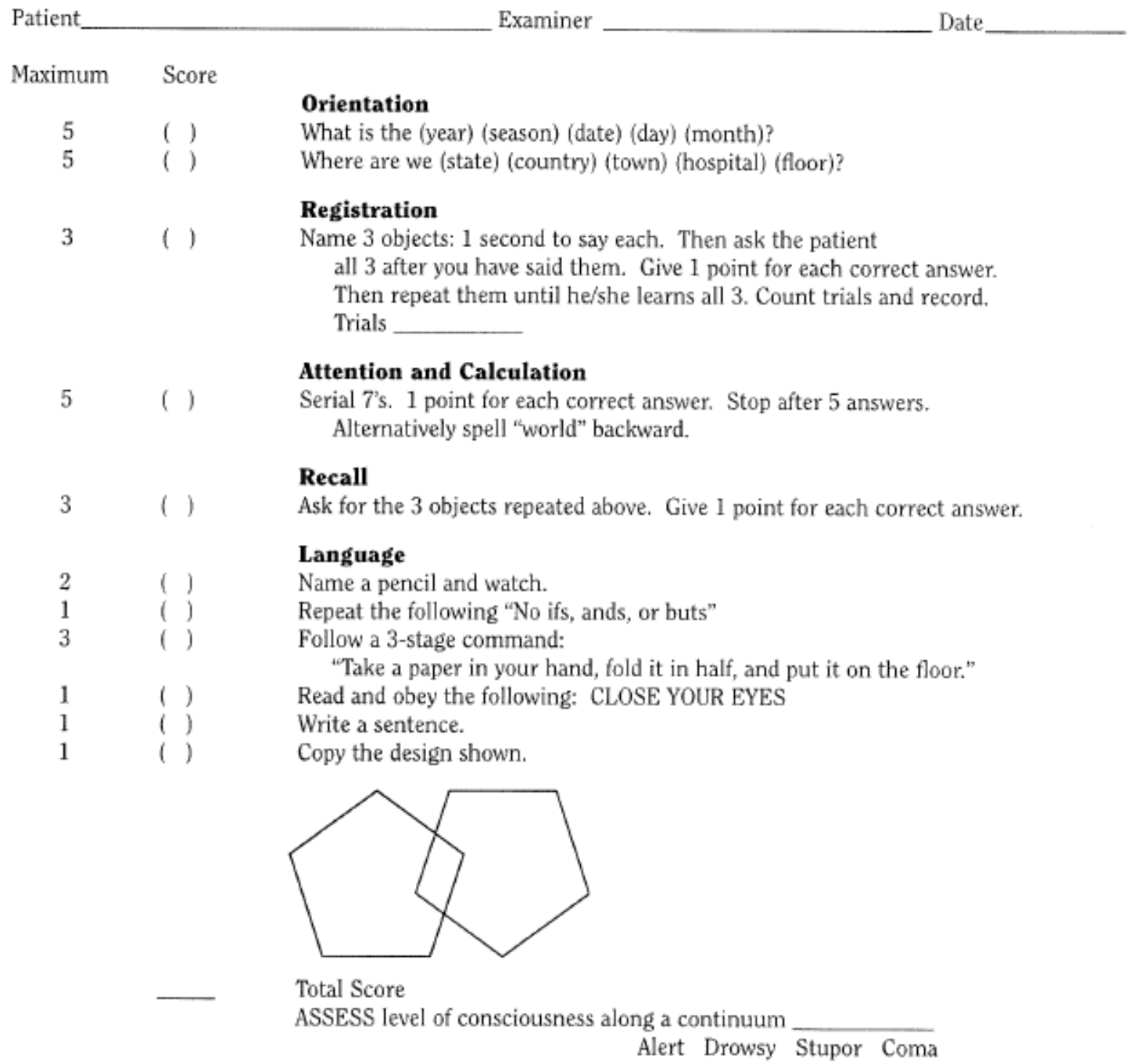


Appendix 4

\section{PHQ-9}

Over the last 2 weeks, how often have you been bothered by any of the following problems?

(Use " $\checkmark$ " to indicate your answer)

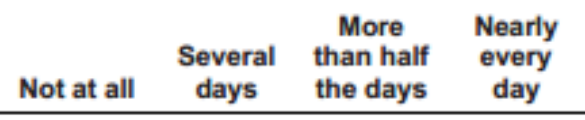

1. Little interest or pleasure in doing things

0

1

2

3

2. Feeling down, depressed, or hopeless

0

1

2

3

3. Trouble falling or staying asleep, or sleeping too much

0

1

2

3

4. Feeling tired or having little energy

0

1

2

3

5. Poor appetite or overeating

0

1

2

3

6. Feeling bad about yourself - or that you are a failure or have let yourself or your family down

0

1

2

3

7. Trouble concentrating on things, such as reading the newspaper or watching television

0

123

8. Moving or speaking so slowly that other people could have noticed? Or the opposite - being so fidgety or restless that you have been moving around a lot more than usual

9. Thoughts that you would be better off dead or of hurting yourself in some way

$\begin{array}{llll}0 & 1 & 2 & 3\end{array}$

$\begin{array}{llll}0 & 1 & 2 & 3\end{array}$

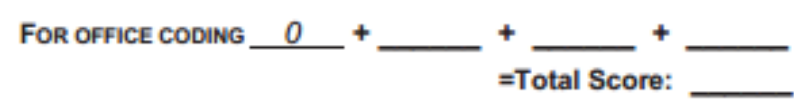

If you checked off any problems, how difficult have these problems made it for you to do your work, take care of things at home, or get along with other people?

\begin{tabular}{|c|c|c|c|}
\hline $\begin{array}{c}\text { Not difficult } \\
\text { at all } \\
\square\end{array}$ & $\begin{array}{c}\text { Somewhat } \\
\text { difficult } \\
\square\end{array}$ & $\begin{array}{c}\text { Very } \\
\text { difficult } \\
\square\end{array}$ & $\begin{array}{c}\text { Extremely } \\
\text { difficult } \\
\square\end{array}$ \\
\hline
\end{tabular}




\begin{tabular}{|c|c|l|}
\hline $\begin{array}{c}\text { PHQ-9 } \\
\text { Score }\end{array}$ & $\begin{array}{c}\text { Depression } \\
\text { Severity }\end{array}$ & Proposed Treatment Actions \\
\hline $0-4$ & None-minimal & None \\
\hline $5-9$ & Mild & Watchful waiting; repeat PHQ-9 at follow-up \\
\hline $10-14$ & Moderate & $\begin{array}{l}\text { Treatment plan, considering counseling, follow-up and/or } \\
\text { pharmacotherapy }\end{array}$ \\
\hline $15-19$ & $\begin{array}{c}\text { Moderately } \\
\text { Severe }\end{array}$ & Active treatment with pharmacotherapy and/or psychotherapy \\
\hline $20-27$ & $\begin{array}{c}\text { Severe } \\
\text { Immediate initiation of pharmacotherapy and, if severe impairment or } \\
\text { poor response to therapy, expedited referral to a mental health } \\
\text { specialist for psychotherapy and/or collaborative management }\end{array}$ \\
\hline
\end{tabular}

* From Kroenke K, Spitzer RL, Psychiatric Annals 2002;32:509-521 
Appendix 5

\section{Stroop Color-Word Test}

\section{STROOP \\ COLOR AND WORD TEST \\ ADULT VERSION}

\begin{tabular}{|c|c|c|c|c|}
\hline \multicolumn{5}{|c|}{ 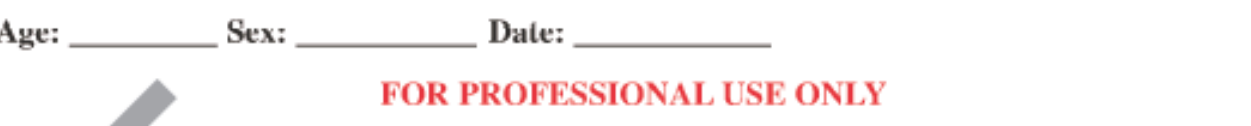 } \\
\hline & $\begin{array}{l}\text { Raw } \\
\text { Score }\end{array}$ & $\begin{array}{l}\text { Age/Ed. } \\
\text { Predicted* }\end{array}$ & Residual & T-Scores*** \\
\hline \multicolumn{5}{|l|}{ Word Score (W) } \\
\hline \multicolumn{5}{|l|}{ Color Score (C) } \\
\hline \multicolumn{5}{|l|}{ Color-Word Score (CW) } \\
\hline $\begin{array}{l}\mathrm{CW}-\text { Predicted = Interference } \\
(\text { Table } \mathrm{V}) \\
\end{array}$ & & & & \\
\hline
\end{tabular}

* This comes from Tables I - III.

** This should come from Table IV or VI. 
Instrument excluded for copyright reasons. 
Instrument excluded for copyright reasons. 
Instrument excluded for copyright reasons. 


\section{Stroop Color-Word Test}

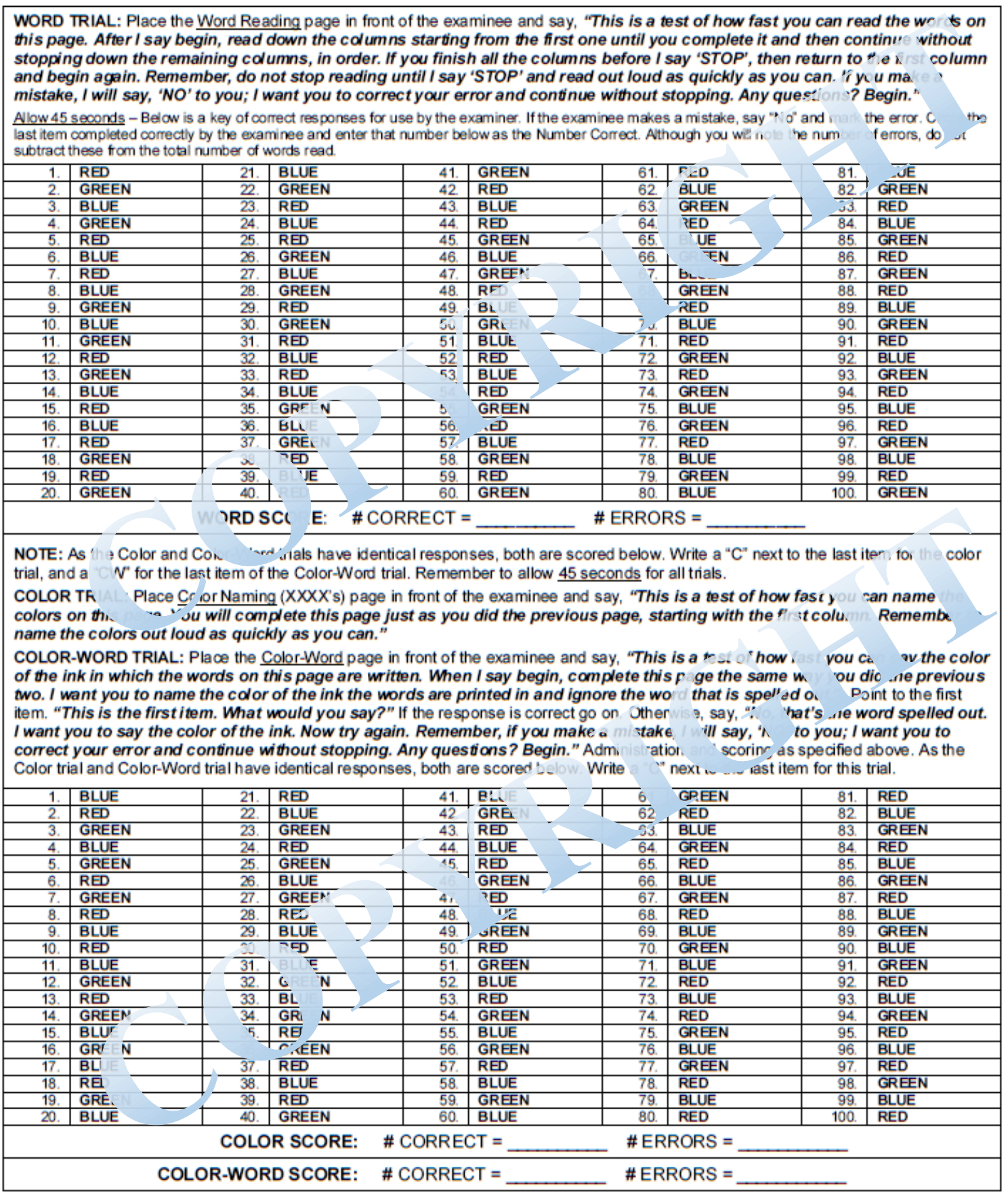


Appendix 6

TMT

\section{Part A}

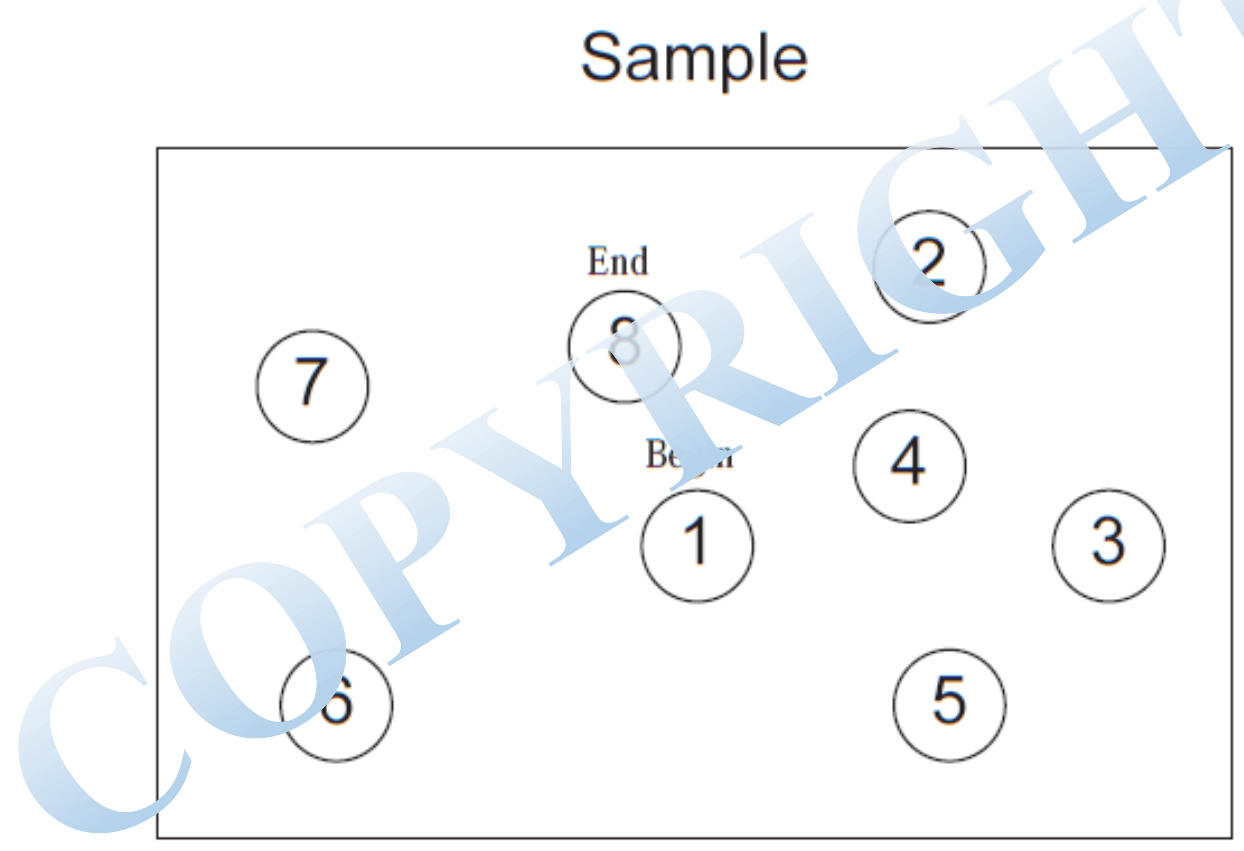


Instrument excluded for copyright reasons. 


\section{TRAIL MAKING Part B}

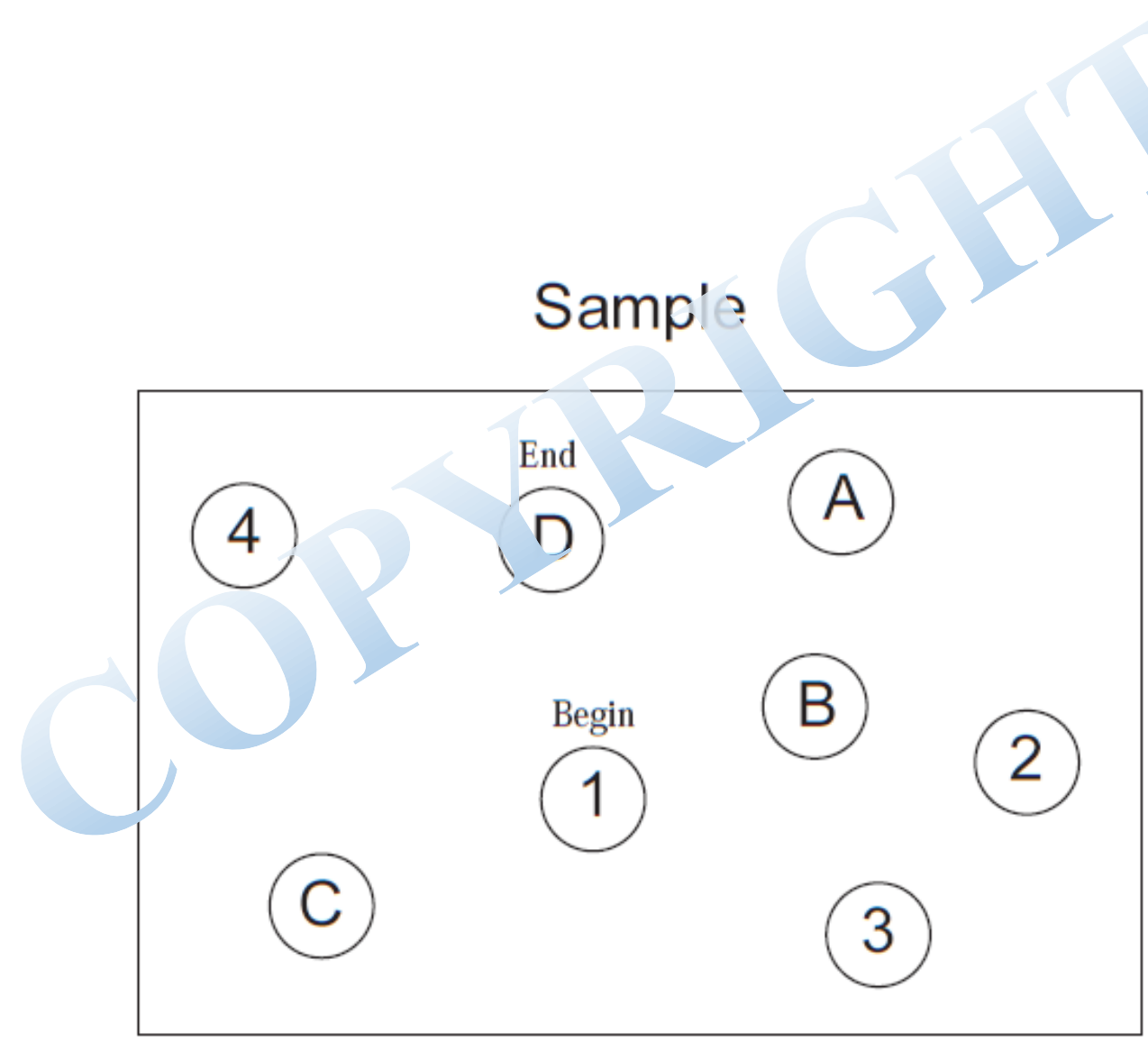


Instrument excluded for copyright reasons. 


\section{General Instructions}

\section{Trail Making Test - Part A}

It is important for the patient to understand that he/she is to work as quickly as possible and avoid making errors. The most common error in administering this test occurs when a subject becomes confused; correct administration procedure requires that the subject be stopped when he/she makes an error and returned to his/her last correct position. This must be done quickly and efficiently as the stopwatch is kept running during this time. Specifically, the subject should not be penalized in his or her time score because of the examiners verbalizations/corrections. Errors count against the subjects performance because the stopwatch is continues to run until the test is completed (or discontinued).

When ready to begin the test, place the Part A test sheet, sample side up, flat on the table directly in front of the subject. The bottom of the test sheet should be approximately six inches from the edge of the table. Give the subject a pencil and SAY:

Sample

"On this page [point] are some numbers. Begin at number one [point to ' 1 '] and draw a line from one to two [point to '2'], two to three [point to ' 3 '], three to four [point to '4'], and so on, in order, until you reach the circle marked end [point to the circle marked 'END']. Draw the lines as fast as you can. Ready? Begin!”

If the subject completes the sample item correctly in a manner demonstrating that he understands what to do, SAY:

\section{“Good! Let's try the next one."}

Turn the page and give part A of the test. If the subject makes a mistake on Sample A, point it out and explain it. The following explanations of mistakes serve as explanations:

1. "You started with wrong circle. This is where you start [point to the circle omitted]."

2. "You skipped this circle* [point to the circle omitted]. You should go from number 1 [point] to 2 [point], 2 to 3 [point], and so on, until you reach the circle marked 'END' [point].

*If it is clear that the subject intended to touch a circle but missed it, do not count it as an omission. Remind the subject, however, to be sure to touch the circles.

If the subject still cannot complete Sample A, take his hand and guide his pencil (using the eraser) though the trail. Then SAY:

SAY:

"NOW YOU TRY IT." Return the pencil to the subject with the point down and

Remember, begin at number one [point to ' 1 '] and draw a line from one to two [point to '2'], two to three [point to ' 3 '], three to four [point to '4'], and so on, in order, until you reach the circle marked end [point to the circle marked 'END']. Draw the lines as fast as you can. Ready? Begin!" 
If the subject succeeds this time, go on to Part A. If not, repeat the procedure until he does succeed or it becomes evident that he/she cannot do the task.

After the subject has completed the sample turn the paper over to Part A and SAY:

\section{PART A:}

"On this page are numbers from 1 to 25. Do this the same way. Begin at number 1 [point], and draw a line from 1 to 2 [point to '2'], 2 to 3 [point to 'three'], 3 to 4 [point to '4'], and so on, in order until you reach the end [point]. Remember, work as fast as you can. Ready? Begin!"

Start timing as soon as the instruction is given to begin. The examiner must watch closely in order to catch any errors as soon as they are made. If the subject makes an error, call it to his attention immediately and have him/her proceed from the point the mistake occurred. Do not stop timing.

After the subject completes Part A, take the test sheet from him/her and record the time in seconds. Errors count only by increasing the performance time.

Next tell the subject: “That's fine. Now we'll try another one." Proceed immediately to Part B, sample.

\section{Trail Making Test - Part B}

Place the test sheet from Part B, sample side up, flat on the table in front of the subject, in the same opposition as the sheet for Part A was placed. Point to the sample and say:

\section{Sample}

"On this page [point] are some numbers and letters. Begin at number 1 [point to ' 1 '] and draw a line from one to $A$ [point to 'A'], $\boldsymbol{A}$ to 2 [point to '2'], 2 to $\boldsymbol{B}$ [point to 'B'], $B$ to 3 [point to ' 3 '], 3 to $C$ [point to ' $C$ '], and so on, in order, until you reach the end [point to the circle marked 'END']. Remember, first you have a number [point to 'I'], then a letter [point to ' $A$ '], then a number [point to ' 2 '], then a letter [point to ' $B$ '], and so on. Draw the lines as fast as you can. Ready? Begin!”

If the subject completes the sample correctly then SAY: “Good! Let's try the next one." Proceed immediately to Part B.

If the subject makes a mistake on Sample B, point it out and explain it. The following explanations of mistakes serve as illustrations: 
1. "You started with wrong circle. This is where you start [point to '1']."

2. "You skipped this circle* [point to the one omitted]. You should go from 1 [point] to A [point], A to 2 [point], 2 to B [point], B to 3 [point], and so on until you reach the circle marked 'END' [point].

*If it is clear that the subject intended to touch a circle but missed it, do not count it as an omission. Remind the subject, however, to be sure to touch the circles.

If the subject still cannot complete Sample B, take his hand and guide his pencil [using the eraser end] though the circles. Then SAY:

"NOW YOU TRY IT. . Begin at number 1 [point to ' 1 '] and draw a line from one to $\boldsymbol{A}$ [point to 'A'], $\boldsymbol{A}$ to 2 [point to '2'], 2 to $\boldsymbol{B}$ [point to 'B'], $\boldsymbol{B}$ to 3 [point to '3'], 3 to $\boldsymbol{C}$ [point to ' $\mathrm{C}$ '], and so on, in order, until you reach the end [point to the circle marked 'END']. Remember, first you have a number [point to '1'], then a letter [point to 'A'], then a number [point to ' 2 '], then a letter [point to ' $\mathrm{B}$ '], and so on. Draw until you reach the circle marked END. Ready? Begin!"

If the subject succeeds this time, go on to Part B, IF not, repeat the procedure until he/she does succeed, or it becomes evident that he/she cannot do the task.

After the subject has completed the sample, turn the paper over to Part B and SAY:

\section{PART B:}

"On this page are both number and letters. Do this the same way. Begin at number 1 [point to ' 1 '] and draw a line from 1 to $A$ [point to 'A'], $\boldsymbol{A}$ to 2 [point to '2'], 2 to $\boldsymbol{B}$ [point to ' $\mathrm{B}$ '], $\boldsymbol{B}$ to 3 [point to ' 3 '], 3 to $C$ [point to ' $C$ '], and so on, in order, until you reach the end [point to the circle marked 'END']. Remember, first you have a number [point to ' 1 '], then a letter [point to 'A'], then a number [point to '2], then a letter [point to ' $B$ '], and so on. Do not skip around, but go from one circle to the next in the proper order, draw the lines as fast as you can. Ready? Begin!"

Start timing as soon as the subject is told to begin. Again, remember to be alert for mistakes. If the subject makes an error, call it to his/her attention immediately and have him proceed from the point the mistake occurred. Do not stop timing.

After the subject completes Part B, take the test sheet from him/her and record the time in seconds. Errors count only by increasing the performance time. 


\section{Appendix 7}

\section{RBANS-U}
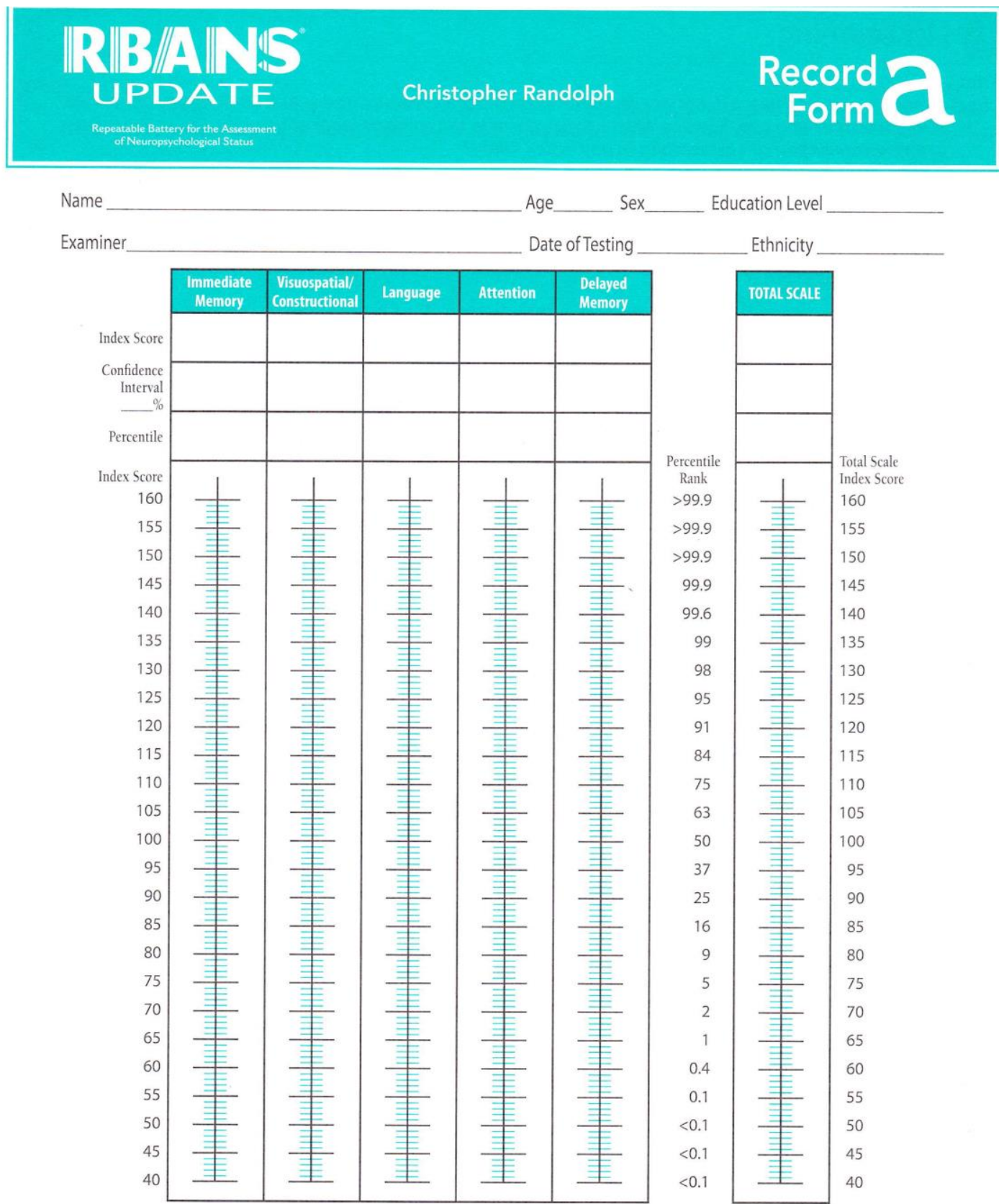

Observations:

\section{PEARSON}

Copyright (c) 1998, 2012 NCS Pearson, Inc. All rights reserved. 
Instrument excluded for copyright reasons. 
Instrument excluded for copyright reasons. 
Instrument excluded for copyright reasons. 
Instrument excluded for copyright reasons. 
Instrument excluded for copyright reasons. 
Instrument excluded for copyright reasons. 
Instrument excluded for copyright reasons. 
Instrument excluded for copyright reasons. 
Instrument excluded for copyright reasons. 
Instrument excluded for copyright reasons. 


\title{
Appendix 8
}

\section{WHOQOL-OLD}

\author{
WHOQOL-OLD (BREF FORMAT)
}

\section{Instructions}

This questionnaire asks for your thoughts and feelings about certain aspects of your quality of life and addresses issues that may be important to you as an older member of society.

Please answer all the questions. If you are unsure about which response to give to a question, please choose the one that appears most appropriate. This can often be your first response.

Please keep in mind your standards, hopes, pleasures and concerns. We ask that you think about your life in the last two weeks.

For example, thinking about the last two weeks, a question might ask:

How much do you worry about what the future might hold?

\begin{tabular}{c|c|c|c|c} 
Not at all & A little & $\begin{array}{c}\text { A moderate } \\
\text { amount }\end{array}$ & Very much & $\begin{array}{c}\text { An extreme } \\
\text { amount }\end{array}$ \\
1 & 2 & 3 & 4 & 5
\end{tabular}

You should circle the number that best fits how much you have worried about the future over the last two weeks. So you would circle the number 4 if you worried about your future "Very much", or circle number 1 if you have worried "Not at all" about your future. Please read each question, assess your feelings, and circle the number on the scale for each question that gives the best answer for you.

Thank you for your help 
The following questions ask about how much you have experienced certain things in the last two weeks.

\begin{tabular}{|c|c|c|c|c|c|c|}
\hline & & Not at all & A little & $\begin{array}{c}\text { A moderate } \\
\text { amount }\end{array}$ & Very mi & $\begin{array}{l}\text { Antreme } \\
\text { ount }\end{array}$ \\
\hline $\begin{array}{l}1 \\
(\mathrm{~F} 25.1)\end{array}$ & $\begin{array}{l}\text { To what extent do impairments to your } \\
\text { senses (e.g. hearing, vision, taste, smell, } \\
\text { touch) affect your daily life? }\end{array}$ & 1 & 2 & 3 & & \\
\hline $\begin{array}{l}2 \\
(\mathrm{~F} 25.3)\end{array}$ & $\begin{array}{l}\text { To what extent does loss of, for example, } \\
\text { hearing, vision, taste, smell or touch affect } \\
\text { your ability to participate in activities? }\end{array}$ & 1 & 2 & 3 & 4 & 5 \\
\hline $\begin{array}{l}3 \\
(\mathrm{~F} 26.1)\end{array}$ & $\begin{array}{l}\text { How much freedom do you have to make } \\
\text { your own decisions? }\end{array}$ & 1 & 2 & 3 & 4 & 5 \\
\hline
\end{tabular}

\begin{tabular}{|c|c|c|c|c|c|c|}
\hline & & Not at $\mathrm{a}$ & intly & Moderately & Very much & Extremely \\
\hline $\begin{array}{l}4 \\
\text { (F26.2) }\end{array}$ & $\begin{array}{l}\text { To what extent do you feel in contro: } \\
\text { your future? }\end{array}$ & 1 & 2 & 3 & 4 & 5 \\
\hline $\begin{array}{l}5 \\
(\mathrm{~F} 26.4)\end{array}$ & $\begin{array}{l}\text { How much do you feel the the pee } \\
\text { around you are respectful or ir f edom? }\end{array}$ & 1 & 2 & 3 & 4 & 5 \\
\hline
\end{tabular}

\begin{tabular}{|c|c|c|c|c|c|c|}
\hline & 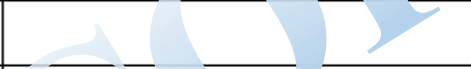 & Not at all & A little & $\begin{array}{c}\text { A moderate } \\
\text { amount }\end{array}$ & Very much & $\begin{array}{c}\text { An extreme } \\
\text { amount }\end{array}$ \\
\hline $\begin{array}{l}6 \\
(\mathrm{~F} 29.2)\end{array}$ & $\begin{array}{l}\text { Tow concern. } \\
\text { hich you will c. }\end{array}$ & 1 & 2 & 3 & 4 & $\sqrt{5}$ \\
\hline
\end{tabular}

\begin{tabular}{|c|c|c|c|c|c|c|}
\hline & $\overline{\overline{2}}=$ & Not at all & Slightly & Moderately & Ven $\overline{\mathrm{ch}}$ & $\overline{E x} \overline{\overline{\text { vely }}}$ \\
\hline $\begin{array}{l}7 \\
(\mathrm{~F} 29.3)\end{array}$ & $\begin{array}{l}\text { How much are you afraid of not being able } \\
\text { to control your death? }\end{array}$ & 1 & 2 & 3 & 4 & \\
\hline $\begin{array}{l}8 \\
(\mathrm{~F} 29.4)\end{array}$ & How scared are you of dying? & 1 & 2 & $3-$ & 4 & 5 \\
\hline
\end{tabular}

\begin{tabular}{|c|c|c|c|c|c|c|}
\hline & & Not at all & $\bar{A}$ i & $\begin{array}{l}\text { oderat } \\
\text { cu...unit }\end{array}$ & Very much & $\begin{array}{c}\text { An extreme } \\
\text { amount }\end{array}$ \\
\hline $\begin{array}{l}9 \\
(\mathrm{~F} 29.5)\end{array}$ & $\begin{array}{l}\text { How much do you fear being in pain before } \\
\text { you die? }\end{array}$ & & 2 & 3 & 4 & 5 \\
\hline
\end{tabular}

The following questions ask about how $\quad \mathbf{D}^{\prime}$. cely you exp rience or were able to do certain things in the last two weeks.

\begin{tabular}{|c|c|c|c|c|c|c|}
\hline & $Y$ & Not at all & A little & Moderately & Mostly & Completely \\
\hline $\begin{array}{l}10 \\
(\mathrm{~F} 25.4)\end{array}$ & 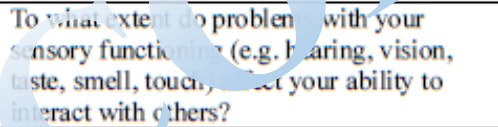 & 1 & 2 & 3 & 4 & 5 \\
\hline $\begin{array}{l}11 \\
(\mathrm{~F} 26.3)\end{array}$ & $\begin{array}{l}\text { hat exte it are you able to do the things } \\
\text { you to do? }\end{array}$ & 1 & 2 & 3 & 4 & 5 \\
\hline $\begin{array}{l}12 \\
(\mathrm{~F} 27.3)\end{array}$ & $\begin{array}{l}\text { To what extent are you satisfied with your } \\
\text { opportunities to continue achieving in life? }\end{array}$ & 1 & 2 & 3 & 4 & 5 \\
\hline $\begin{array}{l}13 \\
(\mathrm{~F} 27.4)\end{array}$ & $\begin{array}{l}\text { How much do you feel that you have } \\
\text { received the recognition you deserve in life? }\end{array}$ & 1 & 2 & 3 & 4 & 5 \\
\hline $\begin{array}{l}14 \\
(\mathrm{~F} 28.4)\end{array}$ & $\begin{array}{l}\text { To what extent do you feel that you have } \\
\text { enough to do each day? }\end{array}$ & 1 & 2 & 3 & 4 & 5 \\
\hline
\end{tabular}


The following questions ask you to say how satisfied, happy or good you have felt about various aspects of your life over the last two weeks.

\begin{tabular}{|c|c|c|c|c|c|c|}
\hline & & $\begin{array}{c}\text { Very } \\
\text { dissatisfied }\end{array}$ & Dissatisfied & $\begin{array}{c}\text { Neither } \\
\text { satisfied nor } \\
\text { dissatisfied }\end{array}$ & Satisfied & St red \\
\hline $\begin{array}{l}15 \\
(\mathrm{~F} 27.5)\end{array}$ & $\begin{array}{l}\text { How satisfied are you with what you have } \\
\text { achieved in life? }\end{array}$ & 1 & 2 & 3 & $\overline{4}$ & 5 \\
\hline $\begin{array}{l}16 \\
(\mathrm{~F} 28.1)\end{array}$ & $\begin{array}{l}\text { How satisfied are you with the way you use } \\
\text { your time? }\end{array}$ & 1 & 2 & 3 & 4 & 5 \\
\hline $\begin{array}{l}18 \\
(\mathrm{~F} 28.7) \\
\end{array}$ & $\begin{array}{l}\text { How satisfied are you with your opportunity } \\
\text { to participate in community activities? }\end{array}$ & 1 & $\overline{2}$ & 3 & 4 & 5 \\
\hline & & $\begin{array}{l}\text { Very } \\
\text { ahappy }\end{array}$ & & $\begin{array}{c}\text { Neither } \\
\text { happy nor } \\
\text { unhappy }\end{array}$ & Happy & Very happy \\
\hline
\end{tabular}

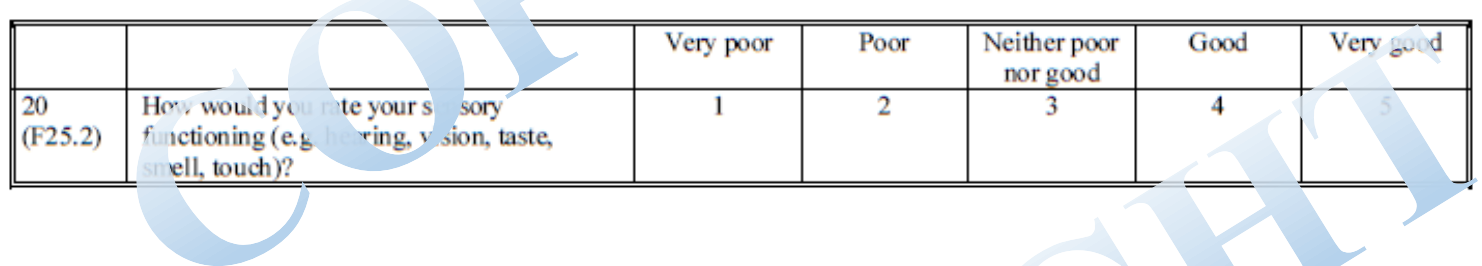

The following question refer to any intimate relationships that you may have. Please cons id er these querion: $h$ reference to a close partner or other close person with whom you can share intimacy more than with any $x$ person our iffe.

\begin{tabular}{|c|c|c|c|c|c|c|}
\hline & & Not at all & 'ittle & $\begin{array}{l}\text { A moderate } \\
\text { rnount }\end{array}$ & Very much & $\begin{array}{l}\text { An extreme } \\
\text { amount }\end{array}$ \\
\hline $\begin{array}{l}21 \\
(\mathrm{~F} 30.2)\end{array}$ & $\begin{array}{l}\text { To what extent do you feel a sense of } \\
\text { companionship in your life? }\end{array}$ & 1 & & 3 & 4 & 5 \\
\hline $\begin{array}{l}22 \\
(\mathrm{~F} 30.3) \\
\end{array}$ & $\begin{array}{l}\text { To what extent do you experience love in } \\
\text { your life? }\end{array}$ & & & & & \\
\hline & & Not at all & A little & Moderately & Mostly & Completely \\
\hline $\begin{array}{l}23 \\
\text { (F30.4) }\end{array}$ & $\begin{array}{l}\text { To what extent you have yortul es to } \\
\text { love? }\end{array}$ & 1 & 2 & 3 & 4 & 5 \\
\hline $\begin{array}{l}24 \\
(\mathrm{~F} 30.7)\end{array}$ & $\begin{array}{l}\text { To what extent de have } \rho \text { portunities to } \\
\text { b oved? }\end{array}$ & & & & & \\
\hline
\end{tabular}

Do you have any comments about the questionnaire?

\section{THANK YOU FOR YOUR HELP}




\section{Scoring}

To score the questionnaire correctly, the following scoring list has to be administered (see Table 7). Scoring the WHOQOL-OLD module involves the following steps:

(a) Basically high scores represent high quality of life, low scores represent low quality of life;

(b) Sorting items into the appropriate scales. For positively worded items, the above classification can be applied in which higher values represent a higher quality of life. For negatively worded items, the score has to be recoded (see below);

(c) Recoding negatively worded items, that is, each item identified with a "*" in the scoring list has to be recoded so that numeric values assigned are reversed: $1=5,2=4$, $3=3,4=2,5=1$. By recoding, high scores in positively worded items reflect higher quality of life. The unidirectional values can subsequently be added to yield the summed scores according to the scoring list of the WHOQOL-OLD module (Table 7);

(d) Using the scoring list (see below) is also necessary for identifying which items belong to a facet. Items to be recoded (see step c) are marked with an "氺";

(e) Summing the items belonging to a facet yields the raw facet score (RFS). Its range lies between the lowest possible (number of items (n) x 1) and highest possible (number of items (n) x 5) value of the respective facet. For the WHOQOL-OLD module, each of the six facets comprises 4 items, thus the lowest possible and the highest possible raw score value are equal across all facets (range 4 to 20);

(f) Comparing scores across facets is possible by directly comparing the raw facet scores. Because every facet comprises of 4 items with the same scaling and scoring format, no transformation of raw scores is needed (so long as all items per facet have been answered); 
(g) If the raw facet score is divided by the number of items in the facet, the resulting standardised (mean) facet score (SFS) can have any decimal value between 1 and 5. A value of 1 represents the lowest possible quality of life rating and a value of 5 the highest possible quality of life rating for the individual;

(h) Transferring a raw score to a transformed scale score (TFS) between 0 and $100 \mathrm{ma}-$ kes it possible to express the scale score in percent between the lowest ( 0$)$ and highest (100) possible value. To obtain the transformed facet score $(0-100)$ the following transformation rule can be applied: TFS $=6.25 \mathrm{x}$ (RFS - 4). (i) Producing the total score of the WHOQOL-OLD involves the addition of the facet scores of a person using all items (instead of the facet items only) of the questionnaire (again paying attention to the recoding procedure - see steps " $\mathrm{b}$ " and "c"). Items may be added to form a total raw score (according to step "e", but using all items), a total standardized (mean) score (according to step "g", but using all items) or a total transformed $(0-100)$ score (according to step "h", but using all items);

(i) Accumulating the values of more than one person - e g. a special age group - simply may be done by summarising the facet and/ or total scores of each individual in that sample (on the level of raw, standardized/ mean or transformed scores) and dividing the respective result through the number of participants to produce the mean score of the respective group.

The above manual scoring approach can be made easier by using a scoring mask that can be easily produced from the scoring list (see step "d"). In addition, the SPSS syntax file (enclosed in Appendix AIII) can be used for the automatic computation of facet and total scores. To work with them, individual data have to be inputted into the computer and identified by variable names and labels, which are also suggested for the enclosed program. When analysing the WHOQOL-OLD module, the facet scores and the total score must be calculated according to the following scoring list (including item numbers for the WHOQOL-OLD module; see Table 7 following). 
Table 7 Scoring list for the WHOQOL-OLD module

\begin{tabular}{|c|c|c|c|c|}
\hline $\begin{array}{l}\text { Sensory Abilities (SAB) } \\
\text { (4 items) }\end{array}$ & old_01* & old_02* & old_10* & old_20 \\
\hline $\begin{array}{l}\text { Autonomy (AUT) } \\
\text { (4 items) }\end{array}$ & old_03 & old_04 & old_05 & old_11 \\
\hline $\begin{array}{l}\text { Past, Present, and Future Abilities (PPF) } \\
\text { (4 items) }\end{array}$ & old_12 & old_13 & old_15 & old_19 \\
\hline $\begin{array}{l}\text { Social Participation (SOP) } \\
\text { (4 items) }\end{array}$ & old_14 & old_16 & old_17 & old_18 \\
\hline $\begin{array}{l}\text { Death and Dying (DAD) } \\
\text { (4 items) }\end{array}$ & old_06* & old_07* & old_08* & old_09* \\
\hline $\begin{array}{l}\text { Intimacy (INT) } \\
\text { (4 items) }\end{array}$ & old_21 & old_22 & old_23 & old_24 \\
\hline Total Score (OLD) & old_01* & old_02* & old_03 & old_04 \\
\hline (24 items) & old_05 & old_06* & old_07* & old_OS* \\
\hline & old_09* & old_10* & old_11 & old_12 \\
\hline & old_13 & old_14 & old_15 & old_16 \\
\hline & old_17 & old_18 & old_19 & old_20 \\
\hline & old_21 & old_22 & old_23 & old_24 \\
\hline
\end{tabular}

Note: * Reverse-scored items. 
Appendix 9

RAND SF-36

1. In general, would you say your health is:

(Circle One Number)

\begin{tabular}{|l|l|}
\hline Excellent & 1 \\
\hline Very good & 2 \\
\hline Good & 3 \\
\hline Fair & 4 \\
\hline Poor & 5 \\
\hline
\end{tabular}

\begin{tabular}{|l|l|c|}
\hline $\begin{array}{l}\text { 2. Compared to one year } \\
\text { ago, how would you rate } \\
\text { your health in general now? }\end{array}$ & \begin{tabular}{l} 
Much better now than one year ago \\
\cline { 2 - 3 }
\end{tabular} & $\begin{array}{l}\text { Somewhat better now than one year } \\
\text { ago }\end{array}$ \\
\cline { 2 - 3 } (Circle One Number) & About the same & 3 \\
\cline { 2 - 3 } & $\begin{array}{l}\text { Somewhat worse now than one year } \\
\text { ago }\end{array}$ \\
\cline { 2 - 3 } & Much worse now than one year ago & 5 \\
\hline
\end{tabular}

The following items are about activities you might do during a typical day. Does your health now limit you in these activities? If so, how much?

(Circle One Number on Each Line) 


\begin{tabular}{|l|c|c|c|}
\hline & $\begin{array}{c}\text { Yes, } \\
\text { Limited } \\
\text { a Lot }\end{array}$ & $\begin{array}{c}\text { Yes, } \\
\text { Limited } \\
\text { a Little }\end{array}$ & $\begin{array}{c}\text { No, } \\
\text { Not } \\
\text { limited } \\
\text { at All }\end{array}$ \\
\hline $\begin{array}{l}\text { 3. Vigorous activities, such as running, } \\
\text { lifting heavy objects, participating in } \\
\text { strenuous sports }\end{array}$ & 1 & 2 & 3 \\
\hline $\begin{array}{l}\text { 4. Moderate activities, such as moving a } \\
\text { table, pushing a vacuum cleaner, bowling, } \\
\text { or playing golf }\end{array}$ & 1 & 2 & 3 \\
\hline 5. Lifting or carrying groceries & 1 & 2 & 3 \\
\hline 6. Climbing several flights of stairs & 1 & 2 & 3 \\
\hline 7. Climbing one flight of stairs & 1 & 2 & 3 \\
\hline 8. Bending, kneeling, or stooping & 1 & 2 & 3 \\
\hline 9. Walking more than a mile & 1 & 2 & 3 \\
\hline 10. Walking several blocks & 1 & 2 & 3 \\
\hline 11. Walking one block & 1 & 2 & 3 \\
\hline 12. Bathing or dressing yourself & 1 & 2 & 3 \\
\hline
\end{tabular}

During the past 4 weeks, have you had any of the following problems with your work or other regular daily activities as a result of your physical health? (Circle One Number on Each Line)

\begin{tabular}{|l|c|c|}
\hline & Yes & No \\
\hline $\begin{array}{l}\text { 13. Cut down the amount of time you spent on work or other } \\
\text { activities }\end{array}$ & 1 & 2 \\
\hline 14. Accomplished less than you would like & 1 & 2 \\
\hline 15. Were limited in the kind of work or other activities & 1 & 2 \\
\hline $\begin{array}{l}\text { 16. Had difficulty performing the work or other activities } \\
\text { (for example, it took extra effort) }\end{array}$ & 1 & 2 \\
\hline
\end{tabular}

During the past 4 weeks, have you had any of the following problems with your work or other regular daily activities as a result of any emotional problems (such as feeling depressed or anxious)? (Circle One Number on Each Line) 


\begin{tabular}{|l|c|c|}
\hline & Yes & No \\
\hline $\begin{array}{l}\text { 17. Cut down the amount of time you spent on work or } \\
\text { other activities }\end{array}$ & 1 & 2 \\
\hline 18. Accomplished less than you would like & 1 & 2 \\
\hline 19. Didn't do work or other activities as carefully as usual & 1 & 2 \\
\hline
\end{tabular}

20. During the past 4 weeks, to what extent has your physical health or emotional problems interfered with your normal social activities with family, friends, neighbors, or groups? (Circle One Number)

\begin{tabular}{|l|l|}
\hline Not at all & 1 \\
\hline Slightly & 2 \\
\hline Moderately & 3 \\
\hline Quite a bit & 4 \\
\hline Extremely & 5 \\
\hline
\end{tabular}

\begin{tabular}{|l|l|l|}
\hline \multirow{4}{*}{$\begin{array}{l}\text { 21. How much bodily pain have you had during } \\
\text { the past } 4 \text { weeks? (Circle One Number) }\end{array}$} & None & 1 \\
\cline { 2 - 3 } & Very mild & 2 \\
\cline { 2 - 3 } & Mild & 3 \\
\cline { 2 - 3 } & Moderate & 4 \\
\cline { 2 - 3 } & Severe & 5 \\
\cline { 2 - 3 } $\begin{array}{l}\text { 22. During the past 4 weeks, how much did } \\
\text { pain interfere with your normal work (including } \\
\text { both work outside the home and housework)? }\end{array}$ & Very severe & 6 \\
\cline { 2 - 3 } (Circle One Number) & A little bit & 2 \\
\cline { 2 - 3 } & Moderately & 3 \\
\hline
\end{tabular}


These questions are about how you feel and how things have been with you during the past 4 weeks. For each question, please give the one answer that comes closest to the way you have been feeling.

How much of the time during the past 4 weeks . . .

(Circle One Number on Each Line)

\begin{tabular}{|l|c|c|c|c|c|c|}
\hline & $\begin{array}{c}\text { All of } \\
\text { the } \\
\text { Time }\end{array}$ & $\begin{array}{c}\text { Most } \\
\text { of } \\
\text { the } \\
\text { Time }\end{array}$ & $\begin{array}{c}\text { A Good } \\
\text { Bit of } \\
\text { the } \\
\text { Time }\end{array}$ & $\begin{array}{c}\text { Some } \\
\text { of the } \\
\text { Time }\end{array}$ & $\begin{array}{c}\text { A } \\
\text { Little } \\
\text { of the } \\
\text { Time }\end{array}$ & $\begin{array}{c}\text { None } \\
\text { of the } \\
\text { Time }\end{array}$ \\
\hline $\begin{array}{l}\text { 23. Did you feel full of } \\
\text { pep? }\end{array}$ & 1 & 2 & 3 & 4 & 5 & 6 \\
\hline $\begin{array}{l}\text { 24. Have you been a } \\
\text { very nervous person? }\end{array}$ & 1 & 2 & 3 & 4 & 5 & 6 \\
\hline $\begin{array}{l}\text { 25. Have you felt so } \\
\text { down in the dumps } \\
\text { that nothing could } \\
\text { cheer you up? }\end{array}$ & 1 & 2 & 3 & 4 & 5 & 6 \\
\hline $\begin{array}{l}\text { 26. Have you felt } \\
\text { calm and peaceful? }\end{array}$ & 1 & 2 & 3 & 4 & 5 & 6 \\
\hline $\begin{array}{l}\text { 27. Did you have a lot } \\
\text { of energy? }\end{array}$ & 1 & 2 & 3 & 4 & 5 & 6 \\
\hline $\begin{array}{l}\text { 28. Have you felt } \\
\text { downhearted and } \\
\text { blue? }\end{array}$ & 1 & 2 & 3 & 4 & 5 & 6 \\
\hline $\begin{array}{l}\text { 29. Did you feel worn } \\
\text { out? }\end{array}$ & 1 & 2 & 3 & 4 & 5 & 6 \\
\hline $\begin{array}{l}\text { 30. Have you been a } \\
\text { happy person? }\end{array}$ & 1 & 2 & 3 & 4 & 5 & 6 \\
\hline \begin{tabular}{l} 
31. Did you feel tired? \\
\hline
\end{tabular} & 1 & 2 & 3 & 4 & 5 & 6 \\
\hline
\end{tabular}


32. During the past $\mathbf{4}$ weeks, how much of the time has your physical health or emotional problems interfered with your social activities (like visiting with friends, relatives, etc.)?

\section{(Circle One Number)}

\begin{tabular}{|l|l|}
\hline All of the time & 1 \\
\hline Most of the time & 2 \\
\hline Some of the time & 3 \\
\hline A little of the time & 4 \\
\hline None of the time & 5 \\
\hline
\end{tabular}

How TRUE or FALSE is each of the following statements for you.

(Circle One Number on Each Line)

\begin{tabular}{|l|c|c|c|c|c|}
\hline & $\begin{array}{c}\text { Definitely } \\
\text { True }\end{array}$ & $\begin{array}{c}\text { Mostly } \\
\text { True }\end{array}$ & $\begin{array}{c}\text { Don't } \\
\text { Know }\end{array}$ & $\begin{array}{c}\text { Mostly } \\
\text { False }\end{array}$ & $\begin{array}{c}\text { Definitely } \\
\text { False }\end{array}$ \\
\hline $\begin{array}{l}\text { 33. I seem to get sick a } \\
\text { little easier than other } \\
\text { people }\end{array}$ & 1 & 2 & 3 & 4 & 5 \\
\hline $\begin{array}{l}\text { 34. I am as healthy as } \\
\text { anybody I know }\end{array}$ & 1 & 2 & 3 & 4 & 5 \\
\hline $\begin{array}{l}\text { 35. I expect my health } \\
\text { to get worse }\end{array}$ & 1 & 2 & 3 & 4 & 5 \\
\hline $\begin{array}{l}\text { 36. My health is } \\
\text { excellent }\end{array}$ & 1 & 2 & 3 & 4 & 5 \\
\hline
\end{tabular}

End of survey

This survey was developed by RAND as part of the Medical Outcomes Study. 


\begin{tabular}{|c|c|c|}
\hline \multirow{3}{*}{ 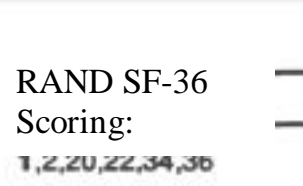 } & \multicolumn{2}{|l|}{ STEP 1: RECODING ITEMS } \\
\hline & Change original response category (a) & To recoded value of: \\
\hline & $1 \longrightarrow$ & 100 \\
\hline & $2 \longrightarrow$ & 75 \\
\hline & $3 \longrightarrow$ & 50 \\
\hline & $4 \longrightarrow$ & 25 \\
\hline & $5 \longrightarrow$ & 0 \\
\hline \multirow[t]{3}{*}{$3,4,5,6,7,8,9,10,11,12$} & $1 \longrightarrow$ & 0 \\
\hline & $2->$ & 50 \\
\hline & $3 \longrightarrow$ & 100 \\
\hline \multirow[t]{2}{*}{$13,14,15,16,17,18,19$} & $1 \longrightarrow$ & 0 \\
\hline & $2 \cdots>$ & 100 \\
\hline \multirow[t]{6}{*}{$21,23,26,27,30$} & $1 \ldots$ & 100 \\
\hline & $2 \cdots>$ & 80 \\
\hline & $3 \longrightarrow>$ & 60 \\
\hline & $4 \longrightarrow$ & 40 \\
\hline & $5 \longrightarrow$ & 20 \\
\hline & $6 \longrightarrow$ & 0 \\
\hline \multirow[t]{6}{*}{$24,25,28,29,31$} & $1 \longrightarrow$ & 0 \\
\hline & $2 \ldots$ & 20 \\
\hline & $3 \longrightarrow$ & 40 \\
\hline & $4 \longrightarrow$ & 60 \\
\hline & $5 \longrightarrow>$ & 80 \\
\hline & $6 \longrightarrow$ & 100 \\
\hline \multirow[t]{5}{*}{$32,33,35$} & $1 \longrightarrow$ & 0 \\
\hline & $2 \longrightarrow$ & 25 \\
\hline & $3 \longrightarrow>$ & 50 \\
\hline & $4 \longrightarrow$ & 75 \\
\hline & $5 \longrightarrow$ & 100 \\
\hline
\end{tabular}

(a) Precoded response choices as printed in the questionnaire. 
STEP 2: AVERAGING ITEMS TO FORM SCALES

\begin{tabular}{lcl}
\hline Scale & $\begin{array}{l}\text { Number Of } \\
\text { ttems }\end{array}$ & $\begin{array}{l}\text { After Recoding Per Table 1, } \\
\text { Average The Following ltems: }\end{array}$ \\
\hline Physical functioning & 10 & 3456789101112 \\
Role limitations due to physical health & 4 & 13141516 \\
Role limitations due to emotional problems & 3 & 171819 \\
Energyffatigue & 4 & 23272931 \\
Emotional well-being & 5 & 2425262830 \\
Social functioning & 2 & 2032 \\
Pain & 2 & 2122 \\
General health & 5 & 133343536
\end{tabular}


Appendix 10

\section{B-ADL}

\section{Bayer-ADL-Scale}

\section{A scale to assess competence in activities of daily living} Informant-rated version

The following questions are about everyday activi $; w$. which she person you are being asked about might have difficulty. Please incin o ho ften unıculties occur by drawing a line through the appropriate circle like this

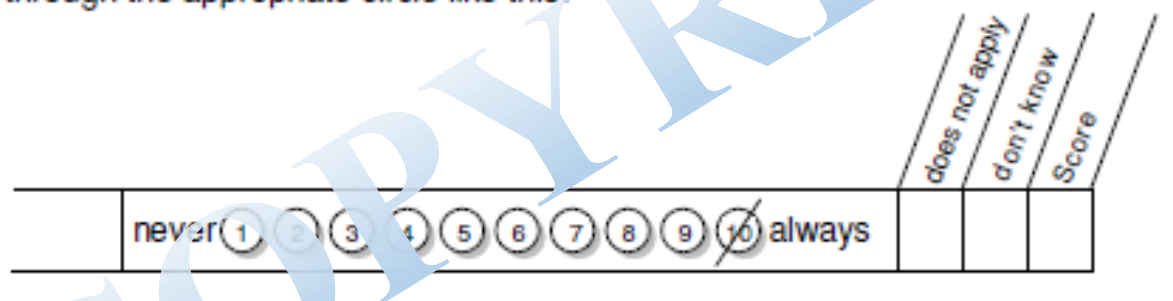

he higher w... -umber, the more difficulty the person has with the activity in question. If ifficulties never occur please mark 1. If difficulties always occur mark 10. If a question does 1. จnn' to the person for any reason please mark the box "does not apply.". If 'ou cannot decide on the difficulty the person has please mark the box "don't know." I ou have problems understanding any of the questions, please ask for help. Pleas to not $v$ ? in the box marked "Score." Thank you very much in advance for your coc peratio,

Name of the person you are being asker abo

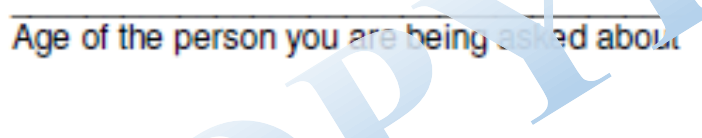

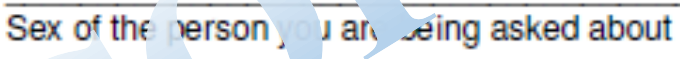


Does the person have difficulty ...

\begin{tabular}{|c|c|c|c|}
\hline $\begin{array}{l}\text { 1. ... managing his/her everyday } \\
\text { activities? }\end{array}$ & never(1) (2) (3) (4) (5) (2) (7) (9) (10) vays & & \\
\hline 2. ... taking care of him/herself? & never (1) (2) (3) (1) (5) (8) (8) (10) always & & \\
\hline $\begin{array}{l}\text { 3. } \cdots \text { taking medication without } \\
\text { supervision? }\end{array}$ & never (1) (2) (3) (4) ( (6) (8) (9) (10) always & & \\
\hline 4. ... with personal hygiene? & never (2) (4) $(5$, (6) (7) (8) (9) (10) always & & \\
\hline $\begin{array}{l}\text { 5. '.. observing important dates or } \\
\text { events? }\end{array}$ & ver(1) (3) (4) (5) (6) (7)(8) (9) (10) always & & \\
\hline 6. ... concentrating on reading & Inev (1) (2) (3) (4) (5) (6) (7)(8)(9) (10) always & & \\
\hline $\begin{array}{l}\text { 7. } \cdots \text { describing what he/ } \\
\text { seen or } h+\text { sar. }\end{array}$ & never (1) (2) (3) (4) (5) (6) (7) (8) (9) (10) always & & \\
\hline 8. ... taking pa t conversation? & never(1) (2) (3) (4) (5) (6) (7) (8) (9) (10) always & & \\
\hline 9 ... using the one? & never(1) (2) (3) (4) (5) (6) (7) (8) (9) (10) always & & \\
\hline 10. taking message for someone & never (1) (2) (3) (4) (5) (6) (7) (8) (9) (10) alwa: & & \\
\hline $\begin{array}{l}\text { 11. .... going for a walk without getting } \\
\text { lost? }\end{array}$ & never (1) (2) (3) (4) (5) (6) (7) (8) (9) (10) a vs & & \\
\hline 12. ... shopping? & (4)(5)(2) (9) alw: & & \\
\hline 13. ... preparing food? & never(1) (2) (3) (4) (5) (7) (8) (10) vays & & \\
\hline 14. ... correctly counting out money? & never (1) (2) (3) (4) (3) (8) (3) (10) always & & \\
\hline $\begin{array}{l}\text { 15. } \cdots \text { understanding his/her personal } \\
\text { financial affairs? }\end{array}$ & never (2) (4) (7) (7) (9) (10) always & & \\
\hline 16. ... giving dire ctions if asked the way? & ne er(1) (3) (5) (6) (7) (9) (9) (10) always & & \\
\hline 17. ... using domestic appliancos? & ir (1) (2) (3) (4) (5) (6) (7) (8) (9) (10) always & & \\
\hline $\begin{array}{l}\text { 18. } . . . \text { finding his/her way in i inf } \\
\text { place? }\end{array}$ & never (1) (2) (3) (4) (5) (6) (7) (9) (9) (10) always & & \\
\hline 19. ... using trar portatio & never (1) (2) (3) (4) (5) (6) (7) (8) (9) (10) always & & \\
\hline $\begin{array}{l}\text { 20. Participat } \\
\text { activities? }\end{array}$ & never(1)(2)(3)(4) (5) (6) (7) (8) (9) (11) always & & \\
\hline $\begin{array}{l}\text { 21. Sontinuinc with the same task after } \\
\text { brief in rruption? }\end{array}$ & never(1) (2) (3) (4) (5) (6) (7) (8) (9) (10) always & & \\
\hline 22. ... doing two things at the same time? & never (1) (2) (3) (4) (5) (6) (7) (8) (9) (10) always & & \\
\hline 23. ... coping with unfamiliar situations? & never (1) (2) (3) (4) (5) (6) (7) (8) (9) (10) always & & \\
\hline 24. ... doing things safely? & never (1)(2) (3) (4) (5) (6) (7) (8) (9) (10) always & & \\
\hline $\begin{array}{l}\text { 25. } \cdots \text { performing a task when under } \\
\text { pressure? }\end{array}$ & never(1) (2) (3) (4) (5) (6) (7) (8) (9) (10) always & & \\
\hline
\end{tabular}


Appendix 11

Manual Log-Sheet and Introduction

\section{Brain Training \\ Log Sheet}

Please use this Log Sheet to record your Brain Training sessions. At the beginning of each session, write the name of the person who is the first one to be the trainer. Remember, each session you are to alternate who gets to be the trainer first. Then, write the day of the week and the date of the session, the time when you start, and the starting page in the manual. At the end of the session, record the time when you are done and the last page you completed. See example below:

\section{Example:}

\begin{tabular}{|l|c|c|c|c|c|c|c|}
\hline \multirow{2}{*}{ Session 1 1} & Trainer first: & Day & Date & Time Begin & Page & Time End & Page \\
\cline { 2 - 8 } & Tom & Tues & $4 / 10 / 15$ & $11: 15 a m$ & 8 & $12: 15 p m$ & 32 \\
\hline \multirow{2}{*}{ Session 2 } & Trainer first: & Day & Date & Time Begin & & Time End & \\
\cline { 2 - 8 } & Mary & Sun & $4 / 15 / 15$ & $3: 30 p m$ & 33 & $4: 30 p m$ & 40 \\
\hline
\end{tabular}

Week 1:

\begin{tabular}{|l|l|l|l|l|l|l|l|}
\hline \multirow{2}{*}{ Session 1 } & Trainer first: & Day & Date & Time Begin & Page & Time End & Page \\
\cline { 2 - 8 } & & & & & & & \\
\hline \multirow{2}{*}{ Session 2 } & Trainer first: & Day & Date & Time Begin & & Time End & \\
\cline { 2 - 8 } & & & & & & & \\
\hline
\end{tabular}

Week 2:

\begin{tabular}{|l|l|l|l|l|l|l|l|}
\hline \multirow{2}{*}{ Session 3} & Trainer first: & Day & Date & Time Begin & Page & Time End & Page \\
\cline { 2 - 9 } & & & & & & & \\
\hline \multirow{2}{*}{ Session 4 } & Trainer first: & Day & Date & Time Begin & & Time End & \\
\cline { 2 - 9 } & & & & & & & \\
\hline
\end{tabular}

[same for Weeks 3-12] 
Dear participants,

Thank you again for agreeing to participate in the research. study. Below you will find an overview of the stud $y$ an ' wha. is expected of you. You will also find instructions on. ow. u use this manual.

\section{The Study}

We developed the $3 r_{c}$ i Tra ing program and would ii e to know how we!! it wo ks. We will evaluate how the progr. n affects your think ig, nemory, and overall ivei heil We are also interest in ihe your thoughts and experie. -es with the prog $n$ so that we can improve it.

We provide all the necessary an als and will assist with any questions or concerns that you ray have.

Your participa ion ill help advance knowledge about aging and vitalit'.

TH, Ikyou! 


\section{Brain Training: Questions and Answers}

\section{Q: What is the Brain Training program?}

A: This program includes many paper-and-penci! task o tra. your memory and thinking. You and your study pa, ner jil take turns training one another and check ing ez h c ner's accuracy. There are no grades or score. 'or oce tasks, but please do your best on each task. If the $\mathrm{t} \cdot \mathrm{k}$ is too hard and/or taking too long, move on to the I xt une.

\section{Q: Where does Bra irainirg take place?}

A: All sessions tá $p$. se at your home or your study partner's home. Ther is $\mathrm{nc}$ travel involved in the study.

\section{Q: Hc In 19 is the Brain Training program?}

A: The program is 12 weeks ( 3 months) long, with two sessions a week ( 24 sessions total). Each session is 1 hour-long.

You and your study partner are to train one anoth for is minutes each. You are provided with a tim er to ${ }^{\prime}$ olp you keep track of time. If you reach the 30-minu $t_{1}$. o lir, it and you are in the middle of a task, comple tha task and then switch roles or end the session.

\section{Q: What do I neea or the Bl din Training program?}

A: You will need is anual, a timer, and a couple of pencils. All of these re pi svided to you. You will also need a quiet area vith a desk or a table and two chairs. It is best that you and $y$. $r$ sudy partner sit across from each other or at two adjacent sides of a table. Please turn off the TV, and silent your phones during the sessions. 


\section{Q: How are the sessions scheduled?}

A: You and your partner schedule the sessions at your mutual convenience twice a week for 12 weeks. We encourage yo ' to discuss and agree ahead of time on days and timest. it wC, $k$ best for the two of you. Please remember co log y ur ; ession days and times in a log-in sheet provided o yo We recommend that you schedule the essuris in the morning and early afternoc $n \mathrm{~W}$ en "nu attention and focus are at their peak. Also, it is best $\mathrm{t}$ choose days of the week that are not too busy o tha you don't feel tired or rushed.

Please avoi havi g sessions two days in a row and allow for at least one 4, ; in between sessions (for example, Tuesc vs

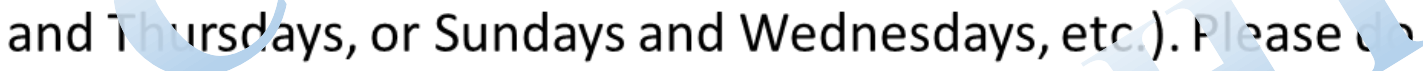
your best to stick to the scheduled days and 'ime Consistency is key! If you have to cancel a sessi in, rease try to reschedule as soon as possible

\section{Q: How does turn-taking work:}

A: You and your partiner $w$. take turns once during each session. Each sessio starts with one of you training the other person. Aft $\mathrm{r} 30$ in tes, you switch roles.

Pleas alternate who gets to train first each session. The log sheet wiil help you keep track of who started as the trainer during the previous session. 


\section{Q: How is this manual used?}

A: The two of you will share one manual. Whoever is the rainer will have the manual in front of him/her and will give task to the other person. After 30 minutes, the trainer gives e mislual to the other person and you switch roles.

Start each session with filling out the $l o$ shcu. It will help you figure out who is to be the tain er rat. nen, the trainer takes the manual and continu s where ou left off.

Each task staris it nstructions for the trainer. The trainer should reac the $i_{1}$; tructions to him/herself silently. Then, the train $r$ shou. rad the directions in blue out loud to his/her partr.

Some tasks have an Answer Sheet that the trainer is o give to the other person to work on. This sheet is labele ass own:

The Answer Sheet always follows the $t$ $k$ inctit is meant for. The trainer should tear it off an $g$ 'o to ris/her partner. Please Keep the used Answer Sheets wi. the manual.

For some exercises, orrect answers are marked or underlined in green so hat $t$ e t. diner can check his/her partner's accuracy. Th, 'rainer has to make sure that the student does not $s$ the correct answers while working on tasks.

Please do your best on all tasks of the Brain Training program, and have fun! 
Appendix 12

Permissions to use Workbook of Activities for Language and Cognition (WALC-2)

\section{and Brainwave-R: Cognitive Strategies and Techniques for Brain Injury}

\section{Rehabilitation.}

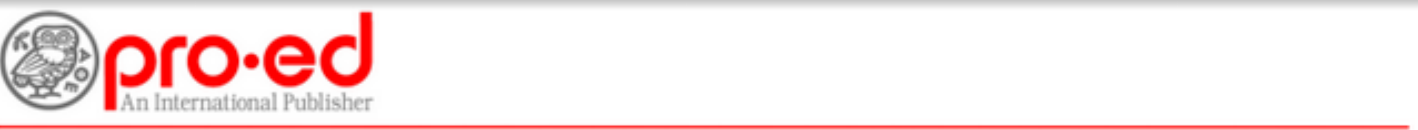

Ms. Natalia Shtompel

July $22^{\text {nd }}, 2014$

Robert Stempel College of Public Health and Social Work

Florida International University

11200 SW $8^{\text {TH }}$ Street, GL475B

Miami, FL 33199

Dear Ms. Natalia Shtompel,

The permission application to reprint All Exercises from [Brainwave-R] Cognitive Strategies and Techniques for Brain Injury Rehabilitation: Visual Processing - Therapist and Client Workbooks in your upcoming SelfFunded Dissertation "Home-based dyadic cognitive training for healthy older adults: A pilots trial" is hereby approved, subject to the payment of the permissions fee.

In accepting this permission, you are agreeing to defend and indemnify PRO-ED, Inc. against any claims resulting from your use of this material.

Permission ID: \#4678 Status: Approved Fee: Electronic copy of dissertation Copies: 20 Pages: All Exercises From title: [Brainwave-R] Cognitive Strategies and Techniques for Brain Injury Rehabilitation: Visual Processing - Therapist and Client Workbooks

Please use the following source note(s):

Thank you for your interest in PRO-ED's publications. Please feel free to contact me if you have any questions regarding this permission.

Best regards,

Yolanda Kiddy,

Permissions, Books \& Materials Division

PRO-ED, Inc. 
․ Kelly Malone<KMalone@linguisystems.com>

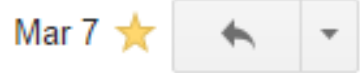

to me $\nabla$

Dear Natalia,

Thank you for writing to request permission to use the WALC materials by Kathryn J. Tomlin in your doctoral study. We're thrilled you've found these materials useful and are happy to grant you permission to use 5-10 full-size pages from any WALC resource in your unpublished dissertation. We ask that the copyright be clearly visible on each page and that you give proper credit to Ms. Tomlin and LinguiSystems in your reference list. Should you decide to publish your thesis, you must reduce the WALC pages by at least $50 \%$.

Best of luck with your study, and we wish you success as you complete your Ph.D.

Sincerely,

Kelly Malone

Permissions 
Appendix 13

\section{Examples of Verbal Memory Tasks.}

Instructions: Read the story slowly to your partner. Next, read the questions and pause after each question to let your partner answer. Repeat the story if needed.

\section{Say: "I am going to read a story.} Listen to the story and answer the questions."

It is common to see thousands of bicyclists or, the stre in Suanghai, China. On the average, 2.5 million bike trips are made daily. Th majo of the motor vehicles are buses and trucks which are used for cor 1 mer I a. assenger service. The drivers in Shanghai are so concerned about hittng a c. 'ist that they continuously use their horns.

\section{Correct Answers:}

1. Name a farniliar s t it hanghai.

2. In what col $v$ is ? nanghai?

3. H many bike trips are made each day?

4. Why do you think there are so many bicycles?

5. What are the predominant motor vehicles?

6. What services do those vehicles provide?

7. What do automobile drivers worry about?

8. How do the drivers warn the bicyc ists?

9. Who rides bikes the most in the ited Siates?

10. What is commercial s ice?

11. Name ano er mec s of transportation.

12. V/ly aren't biu, ies widely used in the United States?

13. WI. A.ie major means of transportation in the United States?

14. When would you use a bicycle?

15. What do you call a three-wheeled cycle?
1. bicyclists

2. China

3. 2.5 million

4. [answeis $\mathrm{h}$, vary]

5. wuses d tru c

6. cc. ercidl and pas, enger service 1. ...titing a cyclist

8. using their horns

9. children

10. service for profit

11. [answers may vary]

12. [answers may vary]

13. car

14. [answers may vary]

15. tricycle 
Instructions: Read the words slowly one line at a time, then read the question. Pause to let your partner answer. Re peat the line if needed.

Correct answers are underlined.

Say: "I am going to read several' wo. 's.

Listen to the words and then in wer question."

1.

$$
\frac{\text { dirt }}{\text { Which ones ar no. weet? }}
$$

2.
cy le
snriek
stick
yell
c. $7 g$
$u$ ich on $ə s$ are not sounds?

3. sun

Which ones are not hot?

fire

iglo

heater

4. bank

$$
\text { beach }
$$

Which ones are not buildiry

5. feathers

Lo ula

hair

car

$\underline{\text { leaf }}$ 
Appendix 14

Examples of Language Tasks.

Instructions: Give the next ANSWER SHEET to your partner.

Say: "In each line, there are words with some ietters missing. The first column includes types of

transportation, and the second colur $n$ in 'ua,s types of clothing. Fill in the missing letters.

Give your partner time to con lete the task. Then, go over the answers to ethe

\section{Correct answers:}

T. nsportation

1. automobile

2. boat

3. motorcycle

4. sleigh

5. airplane

6. ship

7. helicopter

8. coac,

9. iri in

1). horse
Clothing

1. shirt skiı

2. pants

3. $\mathrm{COC}$

1. k elt

5. skirt

6. jacket

7. blouse

8. sweater

9. shoes

10. dress 


\section{Transportation}

\section{Clothing}

1. $a_{-}{ }^{\text {to }}{ }_{-}$obi $-e$

1. s_irt

2. $-0^{+}$

2. _ ant

3. mo - or $-y \_l e$

3. c - at

4. lei $h$

5. $a-r-l a-e$

6.

hi

7. $\mathrm{h}-\mathrm{li}_{-}{ }^{\circ}-$ ter

8. c_ach

4. $b$

5. $-k-k$

6. ia $-k e$

7. - I - use

8. $s w-a-e r$

9. $\quad r a \ldots n$

9. $\mathrm{s}-\mathrm{oe}$

10. $r-r s$

10. d es 
Instructions: Give the next WORK SHEET to your partner. Your partner is to unscramble sentences. If he/she makes a mistake, correct him/her.

Say: "In each line, the words in the sentence are ut of order. Unscramble each sentence and say it th me."

CORRECT A.NSWE 'S:

1. It was aln leav

2. $W$ at re we having for din ner?

3. He called his wife last night; Last night, he called his wife.

4. The leaves change color in autumn; In autumn the leaves change color.

5. He went to coll as in Vermont

6. The ne vs $b$ jins at six coloc.

7. WI en was your last Isntist appointment?

5. The train left from gate three.

9. Buying a house is very expensive.

10. I would like to go home. 


\section{WORK SHEET}

1. to almost it leave time was.

2. having dinner we are for what?

3. night wife he last called his.

4. the in change leaves color auturın.

5. Vermont college went he in to

6. o'clock news the at Jeg. $s$ s.x.

7. dentist whe a poir, nent was your last?

8. the from $t_{\text {t }}$ in iree left gate.

9. buying $a$ is expensive house very.

10. home go I would to like. 
Appendix 15

Examples of Reasoning Tasks.

Instructions: Your partner is to complete some analogies. Read the lines one at a time and pause after each line to let your partner answer. If your partner makes a mistake, correct hi $\mathrm{n} / \mathrm{he}$

Say: Complete these analogies:

1. Metal is to coins as pa jer, to...

1. Bills.

2. Ring is to finger as wa, his to...

2. Wrist.

3. Eves are tc ee as ears are to...

3. Hear.

Hat is to head as gloves are to...

4. Ind,

5. Man is to wallet as woman is to...

5. Purse.

6. Paddle is to canoe as oar is to..

6. Rowboat.

7. Ink is to pen as lead is to...

7. Pencil.

8. Drink is to a gle s a. food is to...

8. A plate.

9. Bo at is to wuier as plane is to...

9. Air.

10. Needle is to pine tree as leave is to...

10. Oak tree, etc.

11. Coffee is to cup as soup is to...

12. Bowl.

12. Stop is to go as red is to...

11. Green. 
Instructions: Your partner is to add a word that belongs with the others. Read the lines one at a time and pause after each, line to let your partner answer.

Say: I am going to say three objects that jeiong $>$ a utegory. Add another object that goes with he $c$ er, and name the category:

\section{EXAMPLES OF CORRECT ANSWERS:}

1. A ton of bricks, lephant, car. $\quad$ 1. A tank/building (heavy)

2. A secon d, a n llimeter, mini-skirt. 2. Inch/chihuahua (short)

3. c rbase, skunk, blue cheese. 3. Rotten food (s melly)

4. Elevator, hot air balloon, steps. $\quad$ 4. Escalator (ta ? yc - up)

5. Attack dog, mosquito, crocodile. 5. $\quad$ ra

6. Rabbit, kangaroo, grasshopper. lee/frog (hop)

7. Scissors, razor blade, ar owhe 7. 7. Knife/needle (sharp)

8. Babies, trees, hair. 8 Nails/grass (grow)

9. Redw od tree, skyscraper, giraffe. 9. Basketball player (tall)

10. Glass, bones, pencil lead. 10. Egg shell (brittle) 
Appendix 16

Examples of Visual Processing tasks

Instructions: Give the next WORK SHEET to your partner.

Say: "Use the coding key in the top part to decode the message in the bottom part."

Give your partner time to finish the tasi. 'T' n, go over the correct answers togethe.

\section{CORRECT ANSWERS:}
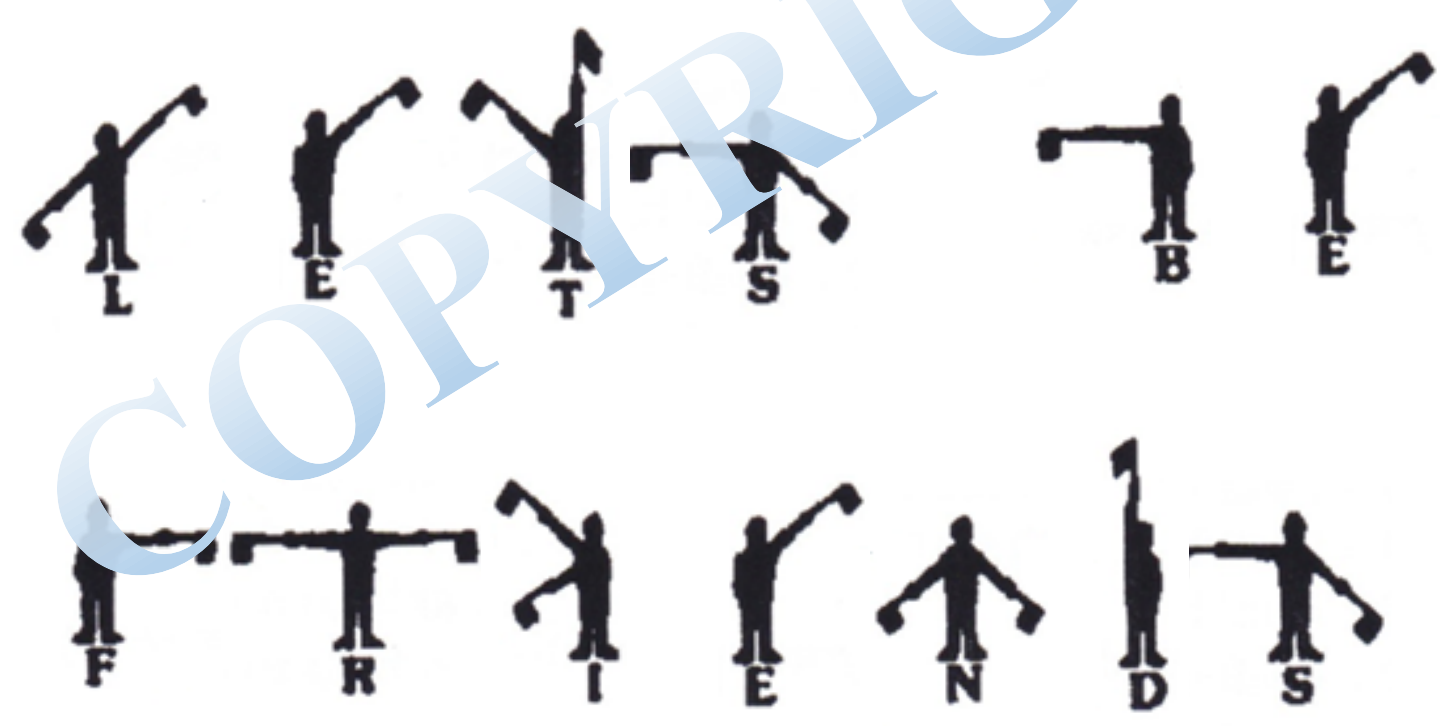

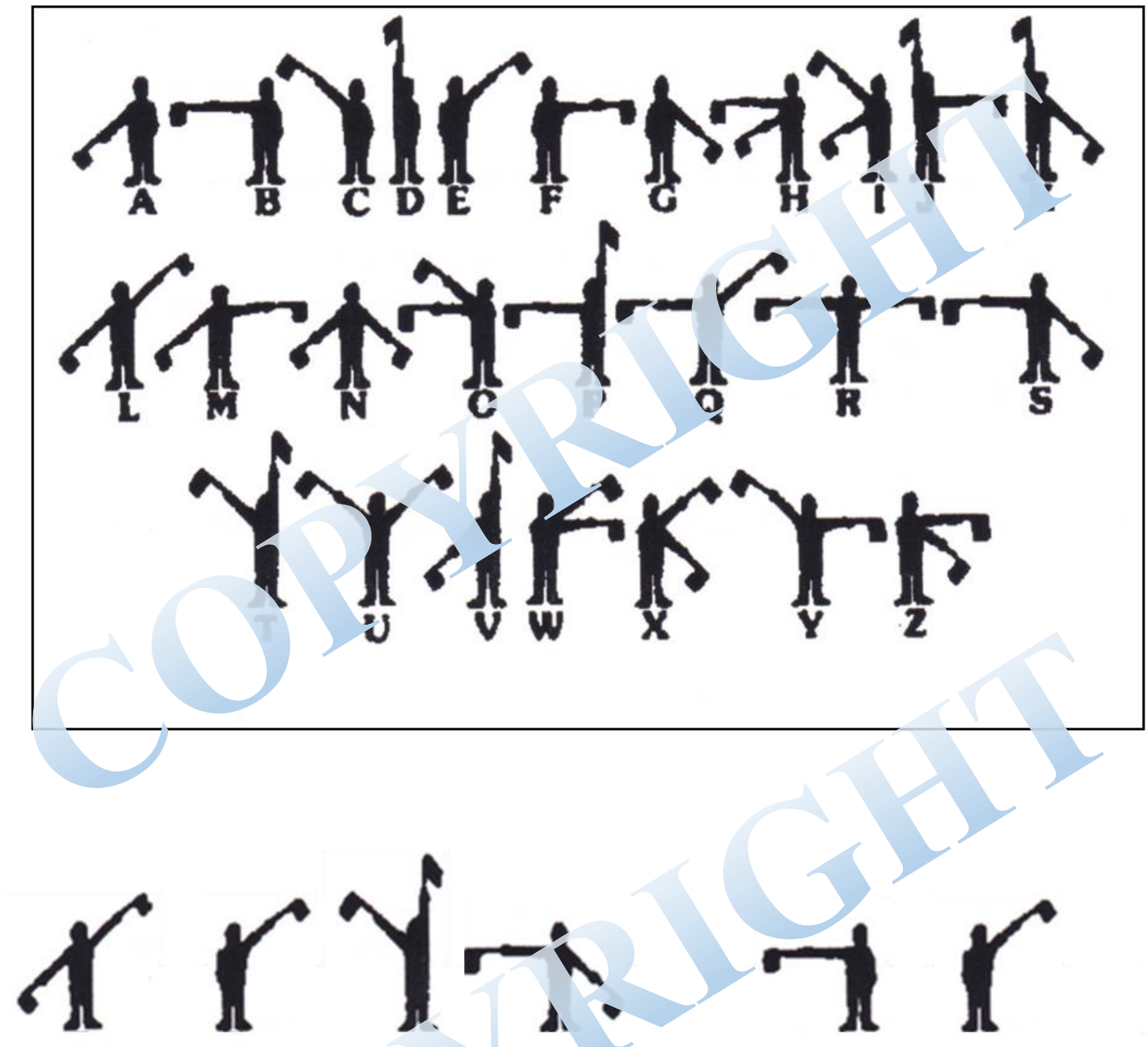

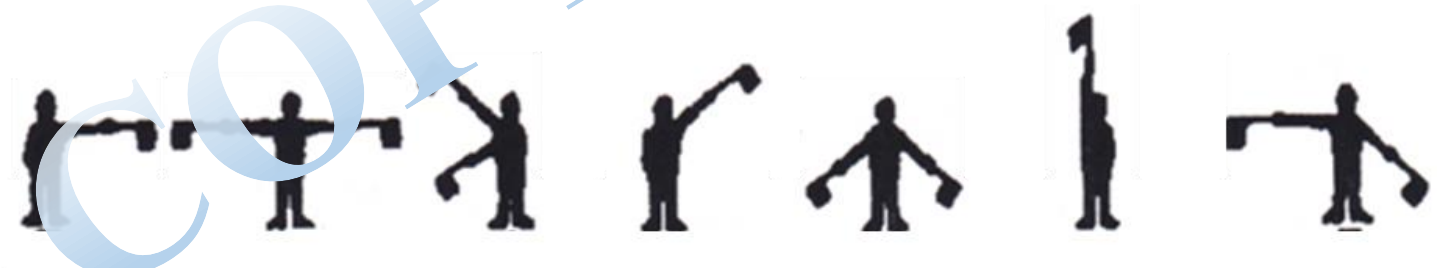


Instructions: Give the next WORK SHEET to your partner.

Say: "There is a key word followed by a line of le te Mark the letters in the line that also appear in $t$ key vord. Mark the letters as many times as they pp. $\mathrm{xr}$ in the line. Then, count how many let ers " u r.arked. Work as fast as you can."

Give your partner time to ce np. ie the task. Then, ask how many letters he/she fo hd ar.al let him/her find any missed letters. Do the ar.ue for cne next word.

\section{CORRECT ANSWERS:}

GARAGE

ELEPHANT
Total Letters I ark

7 Jtal LE .Cers Marked 26 


\section{WORK SHEET}

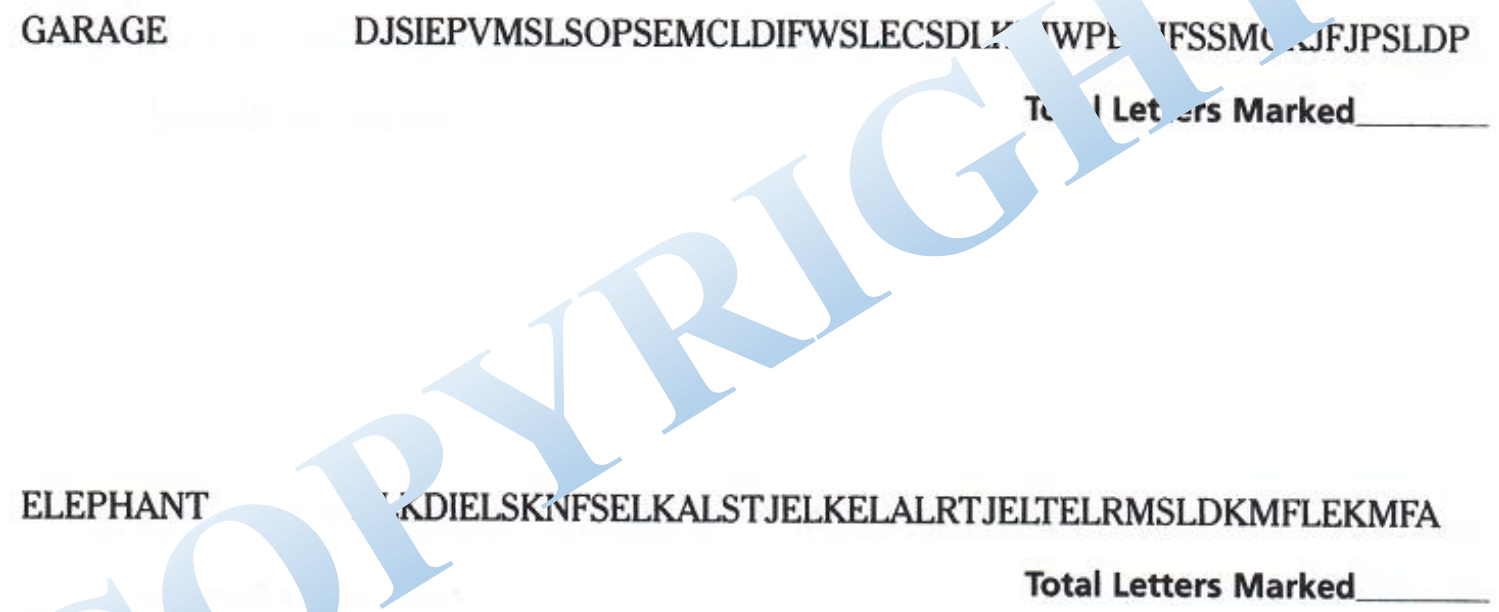


Appendix 17

Study Flyer.

\section{Male and Female Study Participants Needed!}

Are you 65 years old or older?

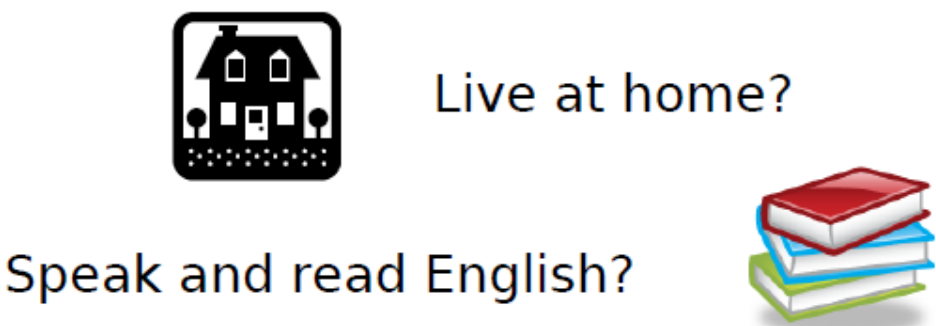

New study at Florida International University (FIU) is recruiting English-speaking volunteers $65+$ years old for a study on memory and thinking.

Where: No need to travel! All parts of the study take place at your home.

How long: FREE 12-week home-based program to train memory and thinking, plus non-intrusive screening and testing at home. All materials provided free of charge, no computer needed.

If interested, please contact:

Natalia Shtompel, M.A., MSW, PhD candidate

(786) 719-0735 Or email: nshto001@fiu.edu 
Appendix 18

Informed Consent Form.

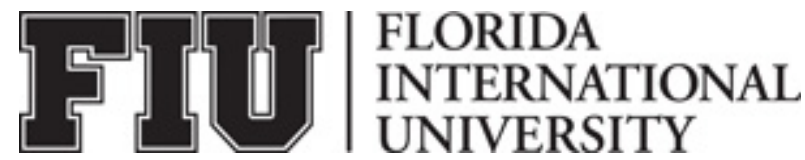

\section{ADULT CONSENT TO PARTICIPATE IN A RESEARCH STUDY BRAIN TRAINING STUDY}

\section{PURPOSE OF THE STUDY}

You are being asked to be in a research study. The purpose of this study is to see how well the Brain Training program works in older adults.

\section{NUMBER OF STUDY PARTICIPANTS}

If you decide to be in this study, you will be one of 20 people in this research study.

\section{DURATION OF THE STUDY}

The duration of the study is about 30 hours over the course of $3-3 \frac{1}{2}$ months.

This includes:

- 1-hour screening.

- 3 hours of testing (two 1.5-hour sessions).

- 24 hours of Brain Training (24 1-hour sessions twice a week for 12 weeks).

- 1-hour interview.

- 30-minute feedback session (optional).

\section{PROCEDURES}

If you agree to participate in the study, we will ask you to do the following things:

1. Screening. Screening will take place today after we finish with this form to confirm that you are eligible for the study. Screening will include some questions about your health and well-being.

It is possible that the results of Screening will show that you are not eligible for the study. Additionally, the researcher is required to make a referral to mental health services or in some cases, contact authorities if it is determined that you may be a danger to yourself or others.

2. First Testing. First Testing will take place at your home. It will follow Screening or will be scheduled for another day. First Testing will include a number of non-intrusive questionnaires and oral or paper-and-pencil tasks that test your thinking and memory. The results may show signs of neurological or mental health issues, in which case appropriate referral will be 
provided. If this occurs, unfortunately you will not be able to continue with the study.

3. Brain Training. The Brain Training program consists of different exercises for your thinking, logic, problem solving, and memory. You will be provided with all materials.

You will be asked to participate in the Brain Training program with your partner. This program is 12 weeks ( 3 months) long. Training sessions are 1-hour long and take place at your home or your partner's home twice a week. It is up to you and your partner which days and times you want to practice.

We will show you how to use the Brain Training manual and work on exercises. We will also check with you regularly over the phone to address any questions or concerns that you may have. The researcher will observe one of your Brain Training sessions to see how things are going.

4. Second Testing. Soon after your last Brain Training session, you will be scheduled for the Second Testing at your home. It will be very similar to the First Testing.

5. Interview. After the Second Testing, you will be asked to undergo an interview or schedule it for another day. The interview will be an informal conversation where you and your partner will be asked to share your experiences and opinions about the Brain Training program. The interviews will be audiorecorded.

6. Optional feedback session. If you are interested, we will schedule a feedback session to discuss the results of your First and Second Testing and the results of the study.

\section{RISKS AND/OR DISCOMFORTS}

No risk of physical harm is associated with this study. The following non-physical risks may be associated with your participation in this study:

First, some questions related to your health and well-being during Screening may be upsetting to you.

Second, it is possible that some tasks during Testing or Brain Training will cause fatigue or frustration. If this happens, you are encouraged to take a break, reschedule the session, or move on to the next task.

Third, it is possible that your participation in Brain Training with your partner will affect your relationship with him or her. 


\section{BENEFITS}

The following benefits are associated with your participation in this study:

First, the study will increase your mental stimulation and social engagement, which is known to be beneficial for older adults.

Second, if you choose to have a feedback session, you will learn about your cognitive strengths and weaknesses as compared to your peers, and possible changes in your thinking and memory over the course of 3 months.

Your participation will benefit the existing scientific knowledge about cognition in older adults and the society as a whole, as it will help determine whether Brain Training program is effective. Ultimately, your participation will contribute to quality of life in older adults and their families.

\section{ALTERNATIVES}

There are no known alternatives available to you other than not taking part in this study. However, any significant new findings developed during the course of the research which may relate to your willingness to continue participation will be provided to you.

\section{CONFIDENTIALITY}

The records of this study will be kept private and will be protected to the fullest extent provided by law. In any sort of report we might publish, we will not include any information that will make it possible to identify a subject. Research records will be stored securely and only the research team will have access to the records. However, your records may be reviewed for audit purposes by authorized University or other agents who will be bound by the same provisions of confidentiality.

Audio-recorded interviews will not be listened to by anyone outside the research team. Quotes from these interviews may appear in published reports without identifying the participants in any way.

A description of this clinical trial will be available on http://www.ClinicalTrials.gov, as required by US Law. This web site will not include information that can identify you. At most, the web site will include a summary of the results. You can search this website at any time.

\section{COMPENSATION \& COSTS}

There is no payment for participants. However, you will receive small nonmonetary incentives 3-4 times throughout the study in appreciation of your participation. You will also receive Brain Training materials free of charge that you can keep after the study. You will not be responsible for any costs in this study. 


\section{MEDICAL TREATMENT}

Routinely, FIU, its agents, or its employees do not compensate for or provide free care for human subjects in the event that any injury results from participation in a research project. If you become ill or injured as a direct result of participating in this study, contact your regular medical provider. If you have insurance, your insurance company may or may not pay for these costs. If you do not have insurance, or if your insurance company refuses to pay, you will be billed. Funds to compensate for pain, expenses, lost wages and other damages caused by injury are not routinely available.

\section{RIGHT TO DECLINE OR WITHDRAW}

Your participation in this study is voluntary. You are free to participate in the study or withdraw your consent at any time during the study. Your withdrawal or lack of participation will not affect any benefits to which you are otherwise entitled. The investigator reserves the right to remove you without your consent at such time that they feel it is in the best interest.

\section{RESEARCHER CONTACT INFORMATION}

If you have any questions about the purpose, procedures, or any other issues relating to this research study you may contact Natalia Shtompel, M.A., MSW at Florida International University, AHC-5 Room 570, 718-219-2884, nshto001@fiu.edu.

\section{IRB CONTACT INFORMATION}

If you would like to talk with someone about your rights of being a subject in this research study or about ethical issues with this research study, you may contact the FIU Office of Research Integrity by phone at 305-348-2494 or by email at ori@fiu.edu.

\section{PARTICIPANT AGREEMENT}

I have read the information in this consent form and agree to participate in this study. I have had a chance to ask any questions I have about this study, and they have been answered for me. I understand that I will be given copy of this form for my records.

Signature of Participant

\section{Date}

Printed Name of Participant

Signature of Person Obtaining Consent

Date 
Appendix 19

\section{In-Person Screening Script.}

ID

1) age [if under 65 , discontinue]

2) town

3) Ethnicity

4) Education

5) Who lives in household?

6) participated in a research study?

7) serious vision or hand problems?

8) hearing problems? [ok if effective hearing aid]

9) Alzheimer's disease, dementia, or mild cognitive impairment (MCI)?

10) serious illness, such as cancer, multiple sclerosis, or stroke? [discontinue if current/recent acute illness with intensive treatment, continue if in remission/medically managed]

11) developmental condition [discontinue if yes]

12) Have you ever had a moderate or severe traumatic brain injury? (if yes, ask for detail)

13) Have you ever had a drowning incident or coma?

14) Have you ever suffered from a psychiatric condition such as depression or bipolar disorder, or substance abuse? [if yes, when? If within past 6 months, discontinue]

15) Other

Eligibility: YES NO

DOB

In US years

Children

Marital statu _Children 
Appendix 20

ID

\section{Qualitative Interview Script.}

Introduction: "Thank you again for your participation in the study. Now I will ask you some questions about the Brain Training program. I will be audio-recording the interview. No one except for the research team will have access to these recordings. We may quote something you say in a presentation or a published report, but we will not include any identifying information. Do you have any questions?"

Questions:

1. Tell me about your experience with the Brain Training program. Prompts: easy/difficult, fun/boring, stimulating/repetitive, enjoyable/frustrating.

2. What was it like being the coach? What was it like being the student?

3. How easy or difficult were the tasks?

4. How easy or difficult was it to use the manual? Prompts: understanding instructions, font, switching roles, keeping track of time, using log sheet, ways to improve the manual.

5. How did the program affect your daily life?

6. How did the program affect your relationship?

7. Did you notice any improvement in your memory or thinking during or after the program?

8. Do you think this program is a good fit for someone like you?

9. How could this program be improved? 


\section{NATALIA SHTOMPEL}

Born, Saint-Petersburg, Russia

2000-2004

2005-2007

2009-2011

$2011-2016$

$1 / 2016-8 / 2016$

$5 / 2016,11 / 2015,10 / 2014$

$11 / 2015,9 / 2014,10 / 2013$

$11 / 2012-6 / 2013$

$8 / 2010-6 / 2011$

$6 / 2011$

$8 / 2006-6 / 2007$

$8 / 2000-5 / 2004$
B.A. Sociology \& Psychology, Salem College, WinstonSalem, NC

M.A. Clinical Psychology, Western Carolina University, Cullowhee, NC

MSW, Master in Social Work, Touro College, New York, NY

Ph.D. in Social Welfare, Florida International University, Miami, FL

Dissertation Year Fellowship (FIU)

Department of Social Work Conference Travel Award (FIU)

Graduate \& Professional Student Committee Conference Travel Award (FIU)

AGE Scholars Fellowship (Miami Area Geriatric Education Center, University of Miami)

Hartford Practicum Partnership Program for Aging Education (HPPAE) Fellowship

Academic Excellence, Outstanding Practicum, \& Community Service Awards (Touro College)

Kendal King Scholarship (Western Carolina University)

Salem College Grant (full scholarship, Salem College)

\section{PUBLICATIONS AND PRESENTATIONS}

Shtompel, N., Ruggiano, N., \& Fortuna, K. (2014). Negative feelings and help seeking among older adults with chronic conditions. Journal of Gerontological Social Work, 57(8), 810-824. 
Ruggiano, N., Shtompel, N., Sias, K., \& Whiteman, K. (2015). Influences of transportation on health decision making and self-management behaviors among older adults with chronic conditions. Behavioral Medicine, 24, 1-9.

Perry, T. E., Ruggiano, N., Shtompel, N., \& Hassevoort, L. A. (2015). Applying Erikson's wisdom to self-management practices of older adults: Findings from two field studies. Research on Aging, 37(3), 253-274.

Ruggiano, N., Shtompel, N., \& Edvardsson, D. (2014). Engaging in coordination of health and disability services as described by older adults: Processes and influential factors. The Gerontologist. doi:10.1093/geront/gnt208

Ruggiano, N., Whiteman, K., \& Shtompel N. (2014). "If I don't like the way I feel with a certain drug, I'll tell them." Older adults' experiences with self-determination and health self-advocacy. Journal of Applied Gerontology. doi:10.1177/0733464814527513

Ruggiano, N., Taliaferro, J. D., \& Shtompel, N. (2013). Interorganizational partnerships as a strategy for lobbying: Nonprofit administrators' perspectives on collaborating for systems-level change. Journal of Community Practice, 21(3), 263-281.

Ruggiano, N., Shtompel, N., Hristidis, V., Roberts, L., Grochowski, J., \& Brown, E. (2012). Need and potential use of information technology for case manager-physician communication in home care. Home Health Care Management Practice, 24(6), 292-297.

Shtompel, N. (2016). Novel, dyadic community-based approach to cognitive training: Results of a pilot study. American Aging Association Annual Meeting (Seattle, WA).

Shtompel, N., Thomlison, B., \& Ruggiano, N. (2015). Older adults training one another: A novel approach to cognitive training. Gerontological Society of America (GSA) Annual Meeting (Orlando, FL).

Shtompel, N., Thomlison, B., \& Ruggiano, N. (2014). Methodological issues in cognition-based interventions for age-related cognitive decline. GSA (Washington, DC).

Ruggiano, N., Fugimoto, K., \& Shtompel, N. (2015). Attributes and role of social networks in facilitating older adults' health literacy and decisions. GSA (Orlando, FL).

Ruggiano, N., Perry, T., Shtompel, N., \& Hassevoort, L. (2014). Using Eriksson's theory to understand health self-management: Findings from two field studies. GSA (Washington, DC).

Ruggiano, N., Shtompel, N., \& Fortuna, K. (2013). More than self-management: Older adults as co-facilitators of care coordination of health and disability services. GSA (New Orleans, LA). 